\title{
DichloroMeldrums Acid (DiCMA): a practical and green amine dichloroacetylation reagent.
}

\author{
David M. Heard, Alastair J.J. Lennox \\ Supplementary Information
}

General Experimental Details.

Synthetic Procedures

Synthesis of DichloroMeldrum's Acid $2 \quad$ S3

Procedure A (Optimisation of reaction conditions) $\quad$ S4

Procedure B: Optimised conditions for the dichloroacetylation of anilines. $\quad$ S9

Procedure C: Optimised conditions for the dichloroacetylation of alkyl amines. $\quad$ S9

$\begin{array}{ll}\text { Characterisation of Products } & \mathrm{S} 10\end{array}$

Preparation of 2,2-dichloro- $N$-(4-fluorophenyl)acetamide $3 \mathbf{a}$ on $5 \mathrm{mmol}$ scale $\quad \mathrm{S} 29$ Investigations into the conversion of $\mathrm{N}$-(4-methylbenzyl)-2,2-dichloroacetamide to $\mathrm{N}$ (4-methylbenzyl)-2,2,2-trichloroacetamide

Preparation of 2,2-dichloro- $\mathrm{N}$-phenylacetamide by amidation with dichloroacetic acid

Unsuccessful Substrates

Comparison of dichloroacetylation with DiCMA versus existing literature conditions, by atom economy and complete E-factor S34

NMR Spectra S39

References 


\section{General Experimental Details.}

All reagents were sourced from commercial suppliers and were used without further purification. Unless otherwise indicated, all reactions were performed open to air, with reagentgrade solvents and no attempt to exclude water. When necessary, dichloromethane, tetrahydrofuran, diethyl ether and toluene were dried using an Anhydrous Engineering alumina column drying system situated in the University of Bristol School of Chemistry. Deuterated solvents for NMR analysis were purchased from Sigma Aldrich. Flash column chromatography was performed according to the procedures used by Still et al. ${ }^{[1]}$ using silica gel 60 (Fisher Scientific or Aldrich) and a suitable eluent. TLC analysis was performed with aluminium backed silica TLC plates (Merck-Kieselgel $60 \mathrm{~F}_{254}$ ) with a suitable solvent system and was visualised using UV fluorescence (254 \& $366 \mathrm{~nm}$ ) and/or developed with potassium permanganate solution.

Infra-red spectra were recorded on a PerkinElmer Spectrum 100 FTIR with an ATR accessory and frequencies are reported in wavenumbers $\left(\mathrm{cm}^{-1}\right) .{ }^{1} \mathrm{H}$ and ${ }^{13} \mathrm{C}$ NMR spectra were recorded using either a Jeol ECS $400 \mathrm{MHz}$ or Varian $400-\mathrm{MR}(400 \mathrm{MHz})$ spectrometer at ambient temperature. Chemical shifts $(\delta)$ are quoted in parts per million (ppm) and coupling constants $(J)$ are in Hertz $(\mathrm{Hz})$, rounded to $0.5 \mathrm{~Hz}$ intervals. Two-dimensional NMR techniques (HSQC, COSY, HMBC) were used routinely for structural assignment. Residual solvent peaks were used as the internal reference for proton and carbon chemical shifts. High-Resolution Mass Spectrometry was performed on a Bruker microTOF II using Electrospray ionisation. Samples were submitted in $\mathrm{MeOH}$, or $\mathrm{CHCl}_{3}$. Specific rotations $\left([a]_{T}^{D}\right)$ were measured on a Bellingham and Stanley Ltd. ADP220 polarimeter and are quoted in $\left({ }^{\circ} \mathrm{ml}\right)(\mathrm{g} \mathrm{dm})^{-1}$.

High-Performance Liquid Chromatography (HPLC) measurements were performed using an Agilent 1260 Infinity instrument, equipped with a diode array detector, measuring at 254 and $280 \mathrm{~nm}$. A ChiralPak® IA column (Amylose tris (3,5-dimethylphenylcarbamate) was used as the stationary phase, with $10 \% 2$-propanol in $n$-hexane used as eluent. 


\section{Synthetic Procedures}

\section{Synthesis of DichloroMeldrum's Acid 2}

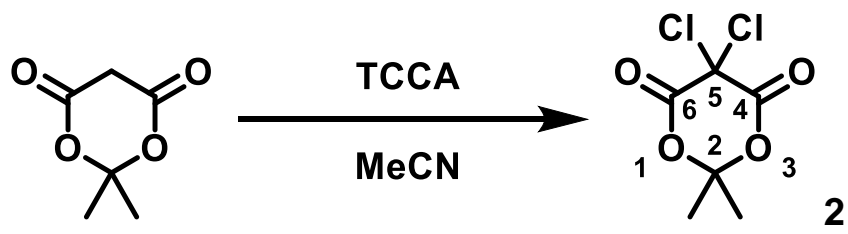

To a stirred solution of meldrum's acid $(10.0 \mathrm{~g}, 69.4 \mathrm{mmol})$ in acetonitrile $(200 \mathrm{~mL})$ at $0{ }^{\circ} \mathrm{C}$ was added trichloroisocyanuric acid (19.4 g, $83.3 \mathrm{mmol}, 1.20$ equiv.) portionwise. After $1 \mathrm{~h}$, the reaction mixture was allowed to warm to room temperature and stirred for $48 \mathrm{~h}$. The reaction mixture was filtered through Celite $\AA$ and concentrated in vacuo. The residue was partitioned between DCM (100 mL) and saturated aqueous $\mathrm{NaHCO}_{3}$ solution $(100 \mathrm{~mL})$. The aqueous phase was extracted with DCM $(2 \times 100 \mathrm{~mL})$, and the combined organic layers dried with $\mathrm{MgSO}_{4}$, filtered, and concentrated in vacuo to yield DichloroMeldrum's acid $\mathbf{2}$ as a colourless oil which crystallises upon standing (13.4 g, 91\%).

DichloroMeldrum's Acid (DiCMA) $\mathbf{2}$ can be stored in an airtight container at room temperature, with no precautions necessary to exclude light. The reagent is stable under these conditions for at least two months, however it is hygroscopic and absorbance of water leads to degradation of the reagent. The toxicity of $\mathbf{2}$ is unknown, and therefore great care should be applied during handling.

${ }^{1} \mathrm{H}$ NMR (400 MHz, $\left.\mathrm{CDCl}_{3}\right) \delta 1.89\left(6 \mathrm{H}, \mathrm{s}, \mathrm{CH}_{3}\right)$

${ }^{13} \mathrm{C}$ NMR (101 MHz, CDCl $) \delta 159.4(\mathrm{C}=\mathrm{O}), 108.1(\mathrm{C} 2), 67.5(\mathrm{C} 5), 28.3\left(\mathrm{CH}_{3}\right)$

These data are consistent with those previously reported. ${ }^{[2]}$ 


\section{Procedure A (Optimisation of reaction conditions)}<smiles>Nc1ccccc1</smiles>

$1 \mathrm{a}$<smiles>CC1(C)OC(=O)C(Cl)(Cl)C(=O)O1</smiles>

2

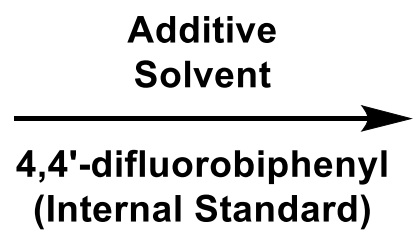

(Internal Standard)<smiles>O=C(Nc1ccc(F)cc1)C(Cl)Cl</smiles>

$3 a$

To a $7 \mathrm{~mL}$ vial equipped with a magnetic stirrer bar was added 4-fluoroaniline 1a $(50.0 \mathrm{mg}$, $0.45 \mathrm{mmol}$ ), DiCMA 2 (1.2 equiv. unless otherwise stated), 4,4'difluorobiphenyl ( $8.5 \mathrm{mg}, 0.045$ $\mathrm{mmol}$ ), solvent ( $2.5 \mathrm{~mL}$ ) and an additive (1.5 equiv., if present). The vial was sealed and stirred at room temperature for $16 \mathrm{~h}$. The resulting solution was then analysed by ${ }^{19} \mathrm{~F}$ NMR. ${ }^{1} \mathrm{NMR}$ yield was determined by comparison of the fluorine signal of the product ( $\delta-118.3 p p m)$ to that of the internal standard ( $\delta-116.5 \mathrm{ppm})$. Mole fraction was determined by ${ }^{19} \mathrm{~F}$ NMR with the following equation:

$$
\text { Mole Fraction } \chi_{3 a}=\frac{\text { Integral of product peak }}{\text { Sum of Integrals of all peaks }}
$$

\footnotetext{
${ }^{1} \mathrm{~A}$ delay time of 2 seconds was used for these ${ }^{19} \mathrm{~F}$ NMR experiments, after it was determined that there was no discernible difference in integral values between when a 2 and a 20 second delay time was applied.
} 


\section{Optimisation of Reaction Time \& Kinetic Analysis}

The reaction was performed according to Procedure $A$, and aliquots removed from the reaction mixture were analysed by ${ }^{19} \mathrm{~F}$ NMR.

$$
[1 a]=[1 a]_{t=0} \times \chi_{1 a}
$$

[1a] vs t.

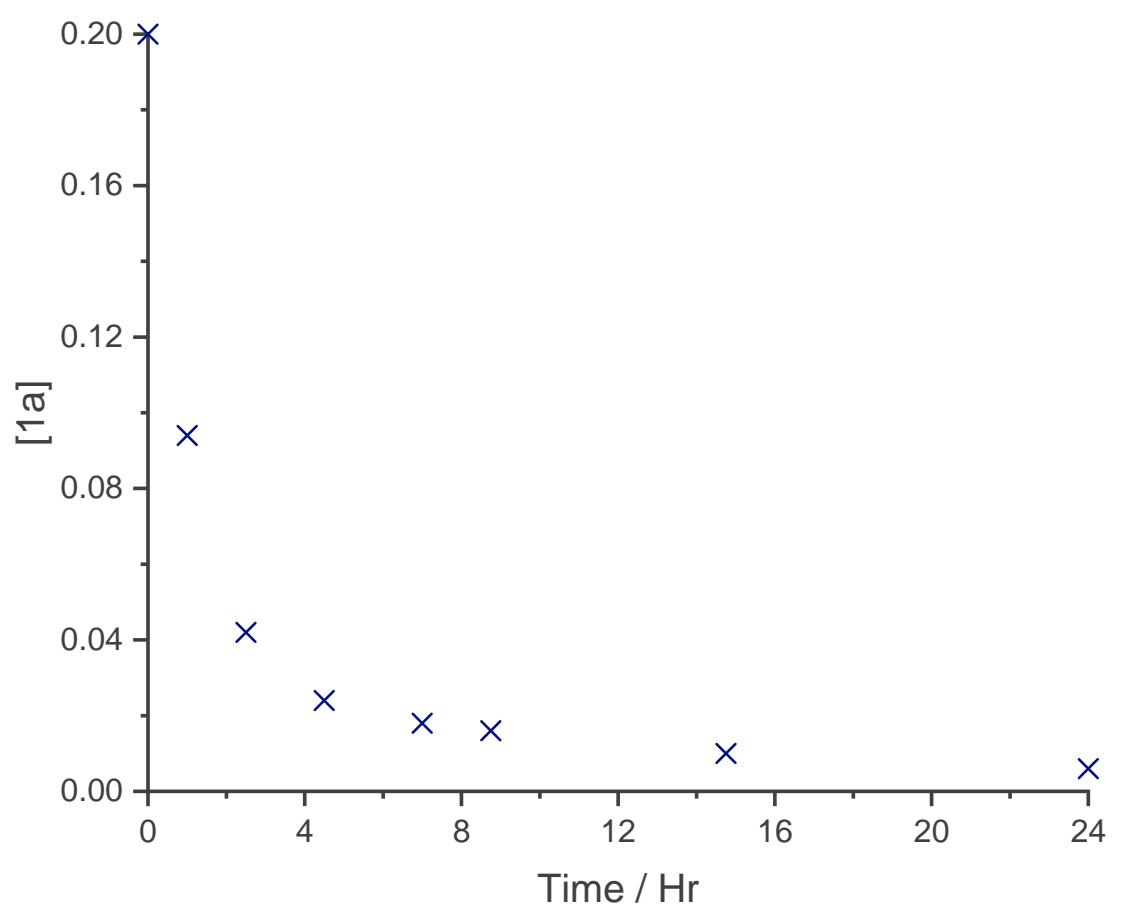


Plot for determination of first order reaction kinetics, In([1a]) vs $t$.

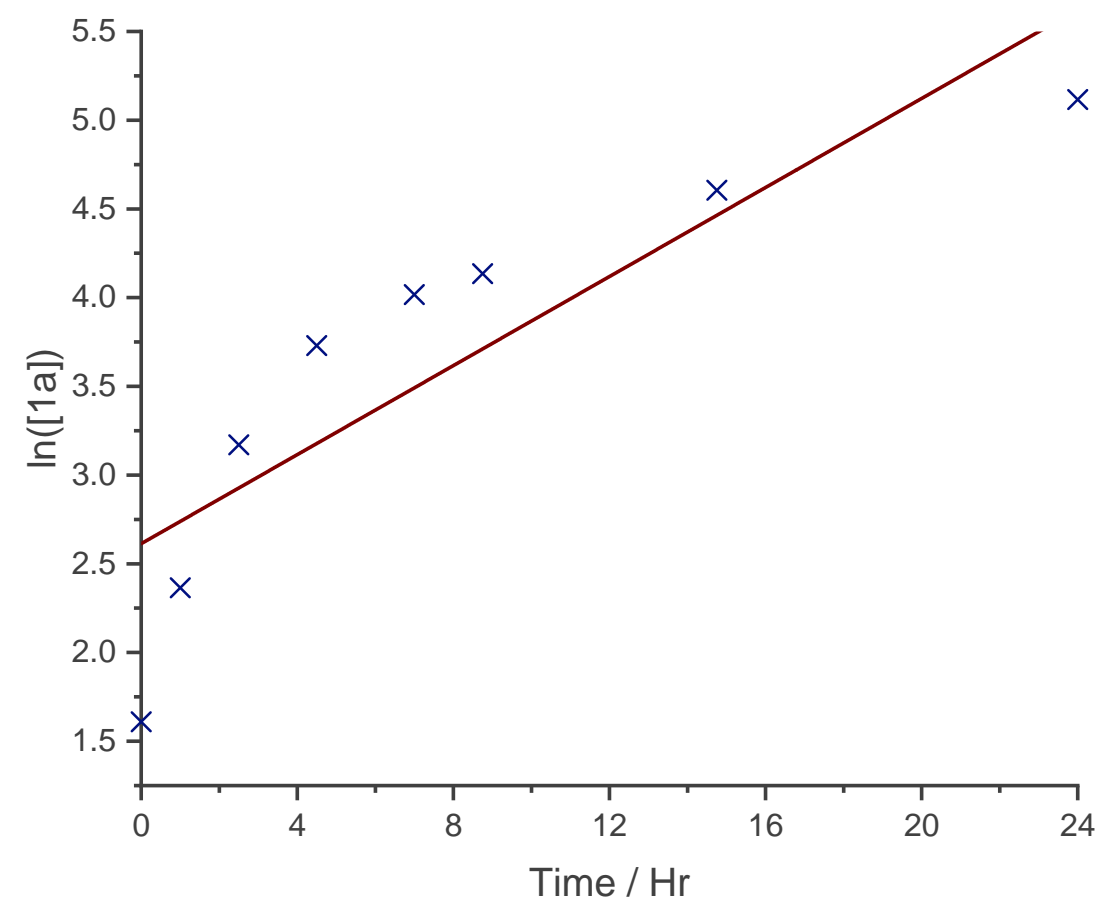

$R^{2}=0.7625$ 
Plot for determination of second order reaction kinetics, 1/[1a] vs $t$.

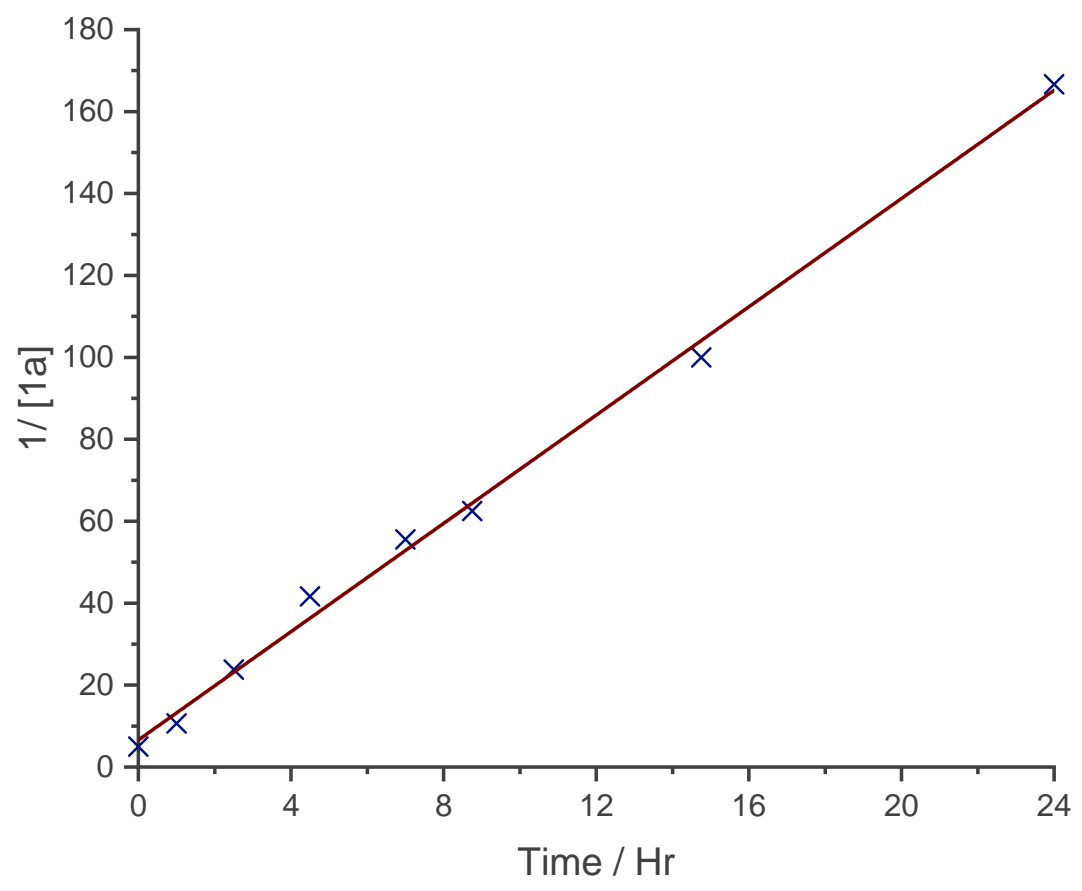

$R^{2}=0.9966$ 


\section{Optimisation of Equivalents of DiCMA}

The reaction was performed according to Procedure A, and the equivalents of DiCMA 2 were varied.

\begin{tabular}{ccc}
\hline Entry & Equivalents & $\chi_{3 a}$ \\
\hline 1 & 1.1 & 0.84 \\
2 & 1.2 & 0.92 \\
3 & 1.5 & 0.97 \\
4 & 2 & 0.99
\end{tabular}

Table S1: Conversion of $1 \mathbf{a}$ to $3 \mathbf{a}$ with different equivalents of DiCMA 2.

\section{Optimisation of Reaction Concentration}

The reaction was performed according to Procedure A, however 2-fluoroaniline $\mathbf{1 g}$ was used as the substrate rather than 4-fluoroaniline 1a, as the conversion was too high with 4fluoroaniline for this to be studied effectively. Whilst very high conversion could be achieved with highly concentrated reaction mixtures, this effect was limited to amines which are liquid at room temperature and dissolve easily in ethyl acetate. Therefore, a concentration of $0.4 \mathrm{M}$ was adopted when studying the substrate scope of the reaction.

\begin{tabular}{ccc}
\hline Entry & $\begin{array}{c}\text { Concentration } \\
/ \mathbf{M}\end{array}$ & $\chi_{\mathbf{3 g}}$ \\
\hline 1 & 0.05 & 0 \\
2 & 0.1 & 0.02 \\
3 & 0.2 & 0.07 \\
4 & 0.5 & 0.46 \\
5 & 1 & 0.82 \\
6 & 2 & 0.95 \\
7 & 4 & 0.98
\end{tabular}

Table S2: Conversion of 1a to $3 \mathbf{a}$ at different concentrations of reaction mixture. 


\section{Procedure B: Optimised conditions for the dichloroacetylation of anilines.}

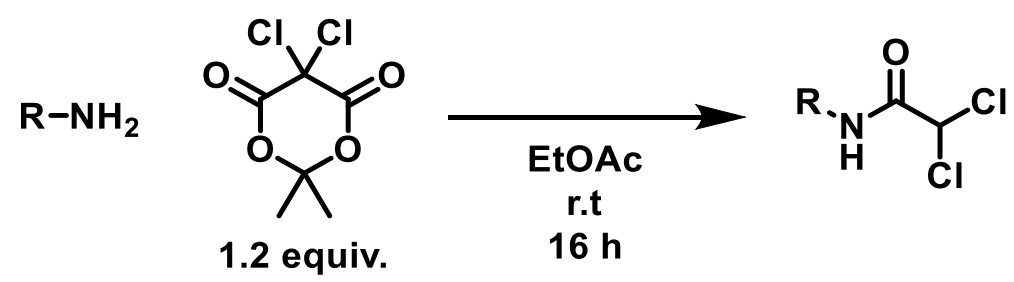

To a $7 \mathrm{~mL}$ vial equipped with a magnetic stirrer bar was added amine $(1.0 \mathrm{mmol})$, ethyl acetate (2.5 mL, $0.40 \mathrm{M}$ ), and then dichloromeldrum's acid (256 mg, $1.20 \mathrm{mmol}, 1.20$ equiv.). The vial was capped and stirred at room temperature for $16 \mathrm{~h}$ and then hydrochloric acid $(2 \mathrm{M}, 10 \mathrm{~mL})$ and diethyl ether $(10 \mathrm{~mL})$ were added. The layers were separated, and the organic layer washed with hydrochloric acid $(2 \mathrm{M}, 10 \mathrm{~mL})$ then saturated aqueous $\mathrm{NaHCO}_{3}(3 \times 10 \mathrm{~mL})$. The organic layer was dried over $\mathrm{MgSO}_{4}$, filtered, and concentrated in vacuo to yield the amide product.

\section{Procedure C: Optimised conditions for the dichloroacetylation of alkyl amines.}<smiles>[R]NN</smiles>

1.2 equiv.<smiles>[R]NC(=O)C(Cl)CCCCC(=O)OCC</smiles>

$16 \mathrm{~h}$

To a $5 \mathrm{~mL}$ round-bottomed flask equipped with a magnetic stirrer bar was added amine (1.0 $\mathrm{mmol}$ ) and ethyl acetate $(1.0 \mathrm{~mL})$. A solution of dichloromeldrum's acid $(256 \mathrm{mg}, 1.20 \mathrm{mmol}$, 1.20 equiv.) in ethyl acetate $(1.0 \mathrm{~mL})$ was then added at a rate of $0.20 \mathrm{~mL} / \mathrm{h}$, controlled by a syringe pump. After the addition was complete, the reaction mixture was stirred at room temperature for $16 \mathrm{~h}$, and then hydrochloric acid $(2.0 \mathrm{M}, 10 \mathrm{~mL})$ and diethyl ether $(10 \mathrm{~mL})$ were added. The layers were separated, and the organic layer washed with hydrochloric acid (2.0 $\mathrm{M}, 10 \mathrm{~mL})$ then saturated aqueous $\mathrm{NaHCO}_{3}(3 \times 10 \mathrm{~mL})$. The organic layer was dried over $\mathrm{MgSO}_{4}$, filtered, and concentrated in vacuo to yield the amide product. 


\section{Characterisation of Products}

\section{2,2-Dichloro- $\mathrm{N}$-(4-fluorophenyl)acetamide 3a}<smiles>O=C(Nc1ccc(F)cc1)C(Cl)Cl</smiles>

Amide 3a was synthesised following Procedure B, from 4-fluoroaniline, to yield a pale brown solid (203 mg, 93\%).

${ }^{1} \mathrm{H}$ NMR (400 MHz, CDCl 3$) \delta 8.13(1 \mathrm{H}, \mathrm{br} \mathrm{s}, \mathrm{NH}), 7.66-7.48(2 \mathrm{H}, \mathrm{m}, \mathrm{Ar}-\mathrm{H}), 7.08(2 \mathrm{H}, \mathrm{t}, J 8.5$ $\mathrm{Hz}, \mathrm{Ar}-\mathrm{H}), 6.04\left(1 \mathrm{H}, \mathrm{s}, \mathrm{CHCl}_{2}\right)$.

${ }^{13} \mathrm{C}$ NMR (101 MHz, CDCl ${ }_{3}$ ) $\delta 162.0$ (C=O), 160.3 (d, J 246.0 Hz, C-F), $158.6(\mathrm{Ar}), 132.3$ (Ar), $122.3(\mathrm{~d}, J 8.0 \mathrm{~Hz}, \mathrm{Ar}), 116.2$ (d, J $23.0 \mathrm{~Hz}, \mathrm{Ar}), 66.1\left(\mathrm{CCl}_{2} \mathrm{H}\right)$.

${ }^{19} \mathrm{~F}\left(376 \mathrm{MHz}, \mathrm{CDCl}_{3}\right) \delta-115.7(\mathrm{Ar}-\mathrm{F})$

These data are consistent with those previously reported. ${ }^{[3]}$

\section{2,2-Dichloro-N-(4-methoxyphenyl)acetamide 3b}<smiles>COc1ccc(NC(=O)C(Cl)Cl)cc1</smiles>

Amide $\mathbf{3 b}$ was synthesised following Procedure $\mathbf{B}$, from $p$-anisidine, to yield a pale brown solid (153 mg, 65\%).

${ }^{1} \mathrm{H}$ NMR (400 MHz, CDCl $)$ ) $8.15(1 \mathrm{H}, \mathrm{br} \mathrm{s}, \mathrm{NH}), 7.45(2 \mathrm{H}, \mathrm{d}, J 9.0 \mathrm{~Hz}, \mathrm{Ar}-\mathrm{H}), 6.89(2 \mathrm{H}, \mathrm{d}, J$ $9.0 \mathrm{~Hz}, \mathrm{Ar}-\mathrm{H}), 6.04\left(1 \mathrm{H}, \mathrm{s}, \mathrm{CHCl}_{2}\right), 3.80(3 \mathrm{H}, \mathrm{s}, \mathrm{OMe})$.

${ }^{13} \mathrm{C}$ NMR (101 MHz, $\left.\mathrm{CDCl}_{3}\right) \delta$ 161.9, 157.5, $129.3(\mathrm{Ar}), 122.3(\mathrm{Ar}), 114.5(\mathrm{Ar}), 67.0\left(\mathrm{CCl}_{2} \mathrm{H}\right.$, $55.6(\mathrm{OMe})$

These data are consistent with those previously reported. ${ }^{[3]}$ 


\section{2,2-Dichloro-N-(4-methylphenyl)acetamide 3c}<smiles>Cc1ccc(NC(=O)C(Cl)Cl)cc1</smiles>

Amide 3c was synthesised following Procedure $\mathbf{B}$, from $p$-toluidine, to yield a brown solid (194 mg, 89\%).

${ }^{1} \mathrm{H}$ NMR $\left(400 \mathrm{MHz}, \mathrm{CDCl}_{3}\right) \delta 8.16(1 \mathrm{H}, \mathrm{s}, \mathrm{NH}), 7.43(2 \mathrm{H}, \mathrm{d}, J 8.5 \mathrm{~Hz}, \mathrm{Ar}-\mathrm{H}), 7.17(2 \mathrm{H}, \mathrm{d}, J 8.5$ $\mathrm{Hz}, \mathrm{Ar}-\mathrm{H}), 6.05\left(1 \mathrm{H}, \mathrm{s}, \mathrm{CHCl}_{2}\right), 2.34(3 \mathrm{H}, \mathrm{s}, \mathrm{Me})$.

${ }^{13} \mathrm{C}$ NMR (101 MHz, $\mathrm{CDCl}_{3}$ ) ठ 161.9 (C=O), 135.7 (Ar), 133.8 (Ar), 129.9 (Ar), $120.4(\mathrm{Ar}), 67.0$ $\left(\mathrm{CHCl}_{2}\right), 21.1(\mathrm{Me})$.

These data are consistent with those previously reported. ${ }^{[4]}$

\section{2,2-Dichloro-N-phenylacetamide 3d}<smiles>O=C(Nc1ccccc1)C(Cl)Cl</smiles>

Amide 3d was synthesised following Procedure B, from aniline, to yield a colourless solid (202 mg, 99\%).

${ }^{1} \mathrm{H}$ NMR (400 MHz, $\left.\mathrm{CDCl}_{3}\right) \delta 8.18(1 \mathrm{H}, \mathrm{s}, \mathrm{NH}), 7.59-7.51(2 \mathrm{H}, \mathrm{m}, \mathrm{Ar}-\mathrm{H}), 7.37(2 \mathrm{H}, \mathrm{dd}, J 8.5$, $7.5 \mathrm{~Hz}, \mathrm{Ar}-\mathrm{H}), 7.20(1 \mathrm{H}, \mathrm{m}, \mathrm{Ar}-\mathrm{H}), 6.05\left(1 \mathrm{H}, \mathrm{s}, \mathrm{CHCl}_{2}\right)$.

${ }^{13} \mathrm{C}$ NMR (101 MHz, $\mathrm{CDCl}_{3}$ ) $\delta 161.9(\mathrm{C}=\mathrm{O}), 136.4(\mathrm{Ar}), 129.4(\mathrm{Ar}), 125.9(\mathrm{Ar}), 120.4(\mathrm{Ar}), 67.0$ $\left(\mathrm{CHCl}_{2}\right)$.

These data are consistent with those previously reported. ${ }^{[3]}$ 


\section{2,2-Dichloro- $N$-(4-trifluoromethylphenyl)acetamide $3 e$}<smiles>O=C(Nc1ccc(C(F)(F)F)cc1)C(Cl)Cl</smiles>

Amide 3e was synthesised following Procedure B, from 4-aminobenzotrifluoride. The product was additionally purified by filtering through a silica plug with $20 \%$ ethyl acetate in hexane, to yield a colourless solid (146 mg, 54\%).

${ }^{1} \mathrm{H}$ NMR (400 MHz, CDCl $)$ $\delta 8.27(1 \mathrm{H}$, br. s, NH), $7.70(2 \mathrm{H}, \mathrm{d}, J 8.5 \mathrm{~Hz}, \mathrm{Ar}-\mathrm{H}), 7.64(2 \mathrm{H}, \mathrm{d}, J$ 8.5, $\mathrm{Ar}-\mathrm{H}), 6.06\left(1 \mathrm{H}, \mathrm{s}, \mathrm{CHCl}_{2}\right)$.

${ }^{13} \mathrm{C}$ NMR (125 MHz, $\mathrm{CDCl}_{3}$ ) $\delta 162.1$ (C=O), 139.4 (q, J $\left.1.5 \mathrm{~Hz}, \mathrm{Ar}\right), 127.7$ (q, J 33.0 Hz, Ar), 126.7 (q, J 4.0 Hz, Ar), 124.0 (q, J272 Hz, CF $)$, 120.1 (Ar), $66.8\left(\mathrm{CHCl}_{2}\right)$.

${ }^{19} \mathrm{~F}\left(376 \mathrm{MHz}, \mathrm{CDCl}_{3}\right) \delta-62.2\left(\mathrm{CF}_{3}\right)$

IR (neat) $\mathbf{V}_{\max } / \mathbf{~ c m}^{-1}:$ 3664, 3245, 2991, 1673, 1605, 1546, 1323, 1109, 1067, 839, 666.

HRMS (APCI) m/z: [M+H+] Calcd for $\mathrm{C}_{9} \mathrm{H}_{6} \mathrm{Cl}_{2} \mathrm{~F}_{3} \mathrm{NO} 271.9851$; Found 271.9851.

N-(4-Benzoylphenyl)-2,2-dichloroacetamide $3 f$<smiles>O=C(c1ccccc1)c1ccc(NC(=O)C(Cl)Cl)cc1</smiles>

Amide 3f was synthesised following Procedure B, from 4-aminobenzophenone and was additionally purified by filtering through a silica plug with $20 \%$ ethyl acetate in hexane, to yield an orange solid (159 mg, 52\%).

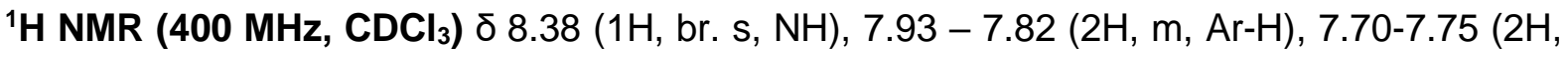
m, Ar-H), $7.72-7.69(2 \mathrm{H}, \mathrm{m}, \mathrm{Ar}-\mathrm{H}), 7.60(1 \mathrm{H}, \mathrm{m}, \mathrm{Ar}-\mathrm{H}), 7.51-7.46(2 \mathrm{H}, \mathrm{m}, \mathrm{ArH}), 6.08(1 \mathrm{H}$, $\left.\mathrm{s}, \mathrm{CHCl}_{2}\right)$.

${ }^{13} \mathrm{C}$ NMR (101 MHz, $\left.\mathrm{CDCl}_{3}\right) \delta 195.7\left(\mathrm{Ph}_{2} \underline{\mathrm{C}}=\mathrm{O}\right), 162.1(\mathrm{C}=\mathrm{O}), 140.2(\mathrm{Ar}), 137.7(\mathrm{Ar}), 134.6(\mathrm{Ar})$, 132.7 (Ar), 131.7 (Ar), 130.1 (Ar), 128.5 (Ar), $119.5(\mathrm{Ar}), 66.9\left(\mathrm{CHCl}_{2}\right)$.

HRMS (ESI) m/z: [M+Na+] Calcd for $\mathrm{C}_{15} \mathrm{H}_{11} \mathrm{Cl}_{2} \mathrm{NNaO}_{2}$ 330.0059; Found 330.0077.

IR (neat) $\mathbf{V}_{\max } / \mathbf{~ c m}^{-1}: 3679,3295,2986,2897,1703,1655,1599,1529,1411,1319,1279$, 1249, 1068, 696. 


\section{2,2-Dichloro- $\mathrm{N}$-(2-fluorophenyl)acetamide $3 \mathrm{~g}$}<smiles>O=C(Nc1ccccc1F)C(Cl)Cl</smiles>

Amide $\mathbf{3 g}$ was synthesised following Procedure $\mathbf{B}$, from 2-fluoroaniline, to yield a pale brown solid (137 mg, 55\%).

${ }^{1} \mathrm{H}$ NMR (400 MHz, $\left.\mathrm{CDCl}_{3}\right) \delta 8.46(1 \mathrm{H}, \mathrm{s}, \mathrm{NH}), 8.22(1 \mathrm{H}, \mathrm{m}, \mathrm{Ar}-\mathrm{H}), 7.22-7.07(3 \mathrm{H}, \mathrm{m}, \mathrm{Ar}-\mathrm{H})$, $6.10\left(1 \mathrm{H}, \mathrm{s}, \mathrm{CHCl}_{2}\right)$.

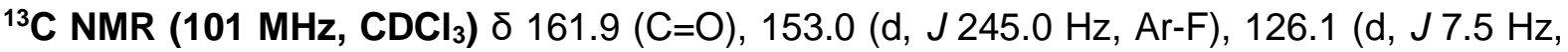
Ar), $125.0(\mathrm{Ar}) 124.8$ (d, J 4.0 Hz, Ar), 121.9 (Ar), 115.2 (d, J 19.0 Hz, Ar), $66.8\left(\mathrm{CHCl}_{2}\right)$.

${ }^{19} \mathrm{~F}\left(376 \mathrm{MHz}, \mathrm{CDCl}_{3}\right) \delta-130.5(\mathrm{Ar}-\mathrm{F})$

These data are consistent with those previously reported. ${ }^{[5]}$

\section{2,2-Dichloro- $\mathrm{N}-(2-c h l o r o p h e n y l) a c e t a m i d e ~ 3 h$}<smiles>O=C(Nc1ccccc1Cl)C(Cl)Cl</smiles>

Amide $3 \mathrm{~h}$ was synthesised following Procedure B, from 2-chloroaniline, to yield a colourless solid (165 mg, 69\%).

${ }^{1} \mathrm{H}$ NMR (400 MHz, CDCl 3$)$ ठ $\left.8.82(1 \mathrm{H}, \mathrm{s}, \mathrm{NH}), 8.30(1 \mathrm{H}, \mathrm{dd}, J 8.0,1.5 \mathrm{~Hz}, \mathrm{Ar}-\mathrm{H})\right), 7.42(1 \mathrm{H}$, dd, J 8.0, 1.5 Hz, Ar-H), 7.32 (1H, ddd, J 8.0, 7.5, 1.5 Hz, Ar-H), 7.13 (1H, ddd, J 8.0, 7.5, 1.5 $\mathrm{Hz}, \mathrm{Ar}-\mathrm{H}), 6.08\left(1 \mathrm{H}, \mathrm{s}, \mathrm{CHCl}_{2}\right)$.

${ }^{13} \mathrm{C}$ NMR (101 MHz, $\left.\mathrm{CDCl}_{3}\right) \delta 161.8(\mathrm{C}=\mathrm{O}), 133.2(\mathrm{Ar}), 129.4(\mathrm{Ar}), 128.0(\mathrm{Ar}), 126.2(\mathrm{Ar}), 123.9$ (Ar), $121.5(\mathrm{Ar}), 67.0\left(\mathrm{CHCl}_{2}\right)$.

These data are consistent with those previously reported. ${ }^{[4]}$ 


\section{2,2-Dichloro- $N$-(2-methoxyphenyl)acetamide $3 i$}<smiles>COc1ccccc1NC(=O)C(Cl)Cl</smiles>

Amide 3i was synthesised following Procedure B, from o-anisidine, to yield a brown solid (222 mg, 95\%).

${ }^{1} \mathrm{H}$ NMR (400 MHz, CDCl 3 ) $\delta 8.89(1 \mathrm{H}, \mathrm{s}, \mathrm{NH}), 8.30(1 \mathrm{H}, \mathrm{dd}, J$ 8.0, $1.5 \mathrm{~Hz}, \mathrm{Ar}-\mathrm{H}), 7.13(1 \mathrm{H}$, ddd, J 8.0, 7.5, 1.5 Hz, Ar-H), 6.99 (1H, td, J 7.5, 1.5 Hz, Ar-H), $6.92(1 \mathrm{H}$, dd, J 8.0, $1.5 \mathrm{~Hz}$, Ar-H), $6.06\left(1 \mathrm{H}, \mathrm{s}, \mathrm{CCl}_{2} \mathrm{H}\right), 3.92(3 \mathrm{H}, \mathrm{s}, \mathrm{OMe})$.

${ }^{13} \mathrm{C}$ NMR (101 MHz, $\mathrm{CDCl}_{3}$ ) $\delta 161.4(\mathrm{C}=\mathrm{O}), 148.5(\mathrm{Ar}), 126.2(\mathrm{Ar}), 125.3(\mathrm{Ar}), 121.2(\mathrm{Ar}), 119.8$ (Ar), 110.3 (Ar), $67.2\left(\mathrm{CHCl}_{2}\right), 56.0(\mathrm{OMe})$.

These data are consistent with those previously reported. ${ }^{[4]}$

\section{2,2-Dichloro- $N$-(2-methylphenyl)acetamide 3j}<smiles>Cc1ccccc1NC(=O)C(Cl)Cl</smiles>

Amide 3j was synthesised following Procedure B, from o-toluidine, to yield a brown solid (168 $\mathrm{mg}, 77 \%)$.

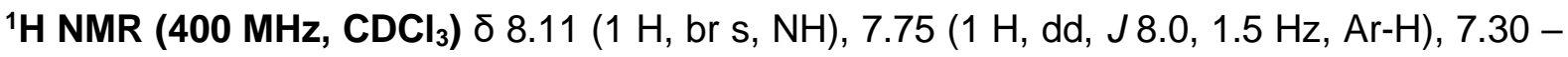
$7.19(2 \mathrm{H}, \mathrm{m}, \mathrm{Ar}-\mathrm{H}), 7.16(1 \mathrm{H}, \mathrm{td}, \mathrm{J} 7.5,1.5 \mathrm{~Hz}, \mathrm{Ar}-\mathrm{H}), 6.07\left(1 \mathrm{H}, \mathrm{s}, \mathrm{CCl}_{2} \mathrm{H}\right), 2.31(3 \mathrm{H}, \mathrm{s}, \mathrm{Me})$ ${ }^{13} \mathrm{C}$ NMR (101 MHz, CDCl 3 ) $\delta 162.8(\mathrm{C}=\mathrm{O}), 139.4(\mathrm{Ar}), 130.9(\mathrm{Ar}), 130.1(\mathrm{Ar}), 127.1(\mathrm{Ar}), 126.6$ (Ar), 123.1 ( $\mathrm{Ar}), 67.1\left(\mathrm{CCl}_{2} \mathrm{H}\right), 19.9(\mathrm{Me})$.

These data are consistent with those previously reported. ${ }^{[4]}$ 


\section{2,2-Dichloro- $N$-(2-ethylphenyl)acetamide 3k}<smiles>CCc1ccccc1NC(=O)C(Cl)Cl</smiles>

Amide 3k was synthesised following Procedure B, from 2-ethylaniline, to yield a colourless solid (171 mg, 74\%).

${ }^{1} \mathrm{H}$ NMR $\left(400 \mathrm{MHz}, \mathrm{CDCl}_{3}\right) \delta 8.18(1 \mathrm{H}, \mathrm{s}, \mathrm{NH}), 7.77(1 \mathrm{H}, \mathrm{d}, J 8.0 \mathrm{~Hz}, \mathrm{Ar}-\mathrm{H}), 7.30-7.14(3 \mathrm{H}, \mathrm{m}$, Ar-H), $6.08\left(1 \mathrm{H}, \mathrm{d}, J 2.0 \mathrm{~Hz}, \mathrm{CHCl}_{2}\right), 2.65\left(2 \mathrm{H}, \mathrm{qd}, J\right.$ 7.5, $\left.2.0 \mathrm{~Hz}, \mathrm{CH}_{2}\right), 1.27(3 \mathrm{H}, \mathrm{td}, J$ 7.5, 2.0 $\left.\mathrm{Hz}, \mathrm{CH}_{3}\right)$.

${ }^{13} \mathrm{C}$ NMR (101 MHz, $\mathrm{CDCl}_{3}$ ) $\delta 162.3$ (C=O), 135.9 (Ar), 133.4 (Ar), 129.0 (Ar), 127.0 (Ar), 126.8 (Ar), $123.5(\mathrm{Ar}), 67.15\left(\mathrm{CHCl}_{2}\right), 24.4\left(\mathrm{CH}_{2}\right), 14.1\left(\mathrm{CH}_{3}\right)$.

HRMS (ESI) m/z: [M+Na+] Calcd for $\mathrm{C}_{10} \mathrm{H}_{11} \mathrm{Cl}_{2} \mathrm{NNaO}$ 254.0110; Found 254.0114.

IR (neat) $\mathbf{V}_{\max } / \mathbf{~ c m}^{-1}: 3675,3253,2971,2901,1671,1588.1546,1452,1337,1250,1066$, $875,809,759$.

\section{2,2-Dichloro- $N$-(2-(4,4,5,5-tetramethyl-1,3,2-dioxaborolan-2-yl)phenyl)acetamide} 3I<smiles>CC1(C)OB(c2ccccc2NC(=O)C(Cl)Cl)OC1(C)C</smiles>

Amide 3I was synthesised following Procedure B, from 2-aminophenylboronic acid pinacol ester, to yield a brown solid (315 mg, 95\%).

${ }^{1} \mathrm{H}$ NMR $\left(400 \mathrm{MHz}, \mathrm{CDCl}_{3}\right) \delta 10.51(1 \mathrm{H}, \mathrm{s}, \mathrm{NH}), 8.46(1 \mathrm{H}, \mathrm{d}, J 8.5 \mathrm{~Hz}, \mathrm{Ar}-\mathrm{H}), 7.82(1 \mathrm{H}, \mathrm{dd}, J$ 7.5, $2.0 \mathrm{~Hz}, \mathrm{Ar}-\mathrm{H})$ ), $7.49(1 \mathrm{H}$, ddd, J 8.5, 7.5, $2.0 \mathrm{~Hz}, \mathrm{Ar}-\mathrm{H}), 7.15(1 \mathrm{H}, \mathrm{t}, J 7.5 \mathrm{~Hz}, \mathrm{Ar}-\mathrm{H}), 6.05$ $\left(1 \mathrm{H}, \mathrm{s}, \mathrm{CHCl}_{2}\right), 1.39(12 \mathrm{H}, \mathrm{s}, 4 \times \mathrm{Me})$.

${ }^{13} \mathrm{C}$ NMR (101 MHz, CDCl 3 ) $\delta 162.2(\mathrm{C}=\mathrm{O}), 143.5(\mathrm{Ar}), 136.3(\mathrm{Ar}), 132.9(\mathrm{Ar}), 124.1(\mathrm{Ar}), 119.2$ (Ar), 117.0 (br, Ar), 84.8 (C-O), $67.6\left(\mathrm{CHCl}_{2}\right), 24.9(4 \times \mathrm{Me})$.

HRMS (ESI) m/z: [M+Na+] Calcd for $\mathrm{C}_{14} \mathrm{H}_{18} \mathrm{BCl}_{2} \mathrm{NNaO}_{3} 352.0652$; Found 352.0660.

IR (neat) $\mathbf{V}_{\max } / \mathbf{~ c m}^{-1}: 3675,3312,2986,2901,1695,1583,1547,1449,1383,1354,1215$, 1074,754 
2,2-Dichloro- $N$-(naphthalen-1-yl)acetamide 3m<smiles>O=C(Nc1cccc2ccccc12)C(Cl)Cl</smiles>

Amide 3m was synthesised following Procedure B, from 1-naphthylamine, to yield a grey solid (215 mg, 85\%).

${ }^{1} \mathrm{H}$ NMR $\left(400 \mathrm{MHz}, \mathrm{CDCl}_{3}\right) \delta 8.57(1 \mathrm{H}, \mathrm{s}, \mathrm{NH}), 7.93-7.85(3 \mathrm{H}, \mathrm{m}, \mathrm{Ar}-\mathrm{H}), 7.79(1 \mathrm{H}, \mathrm{d}, J 8.0 \mathrm{~Hz}$, $\operatorname{Ar}-\mathrm{H}), 7.62-7.51(3 \mathrm{H}, \mathrm{m}, \mathrm{Ar}-\mathrm{H}), 6.18\left(1 \mathrm{H}, \mathrm{s}, \mathrm{CHCl}_{2}\right)$.

${ }^{13} \mathrm{C}$ NMR (101 MHz, $\mathrm{CDCl}_{3}$ ) $\delta 162.7(\mathrm{C}=\mathrm{O}), 134.3(\mathrm{Ar}), 130.6(\mathrm{Ar}), 129.1(\mathrm{Ar}), 127.4(\mathrm{Ar}), 127.3$ (Ar), 127.1 (Ar), 126.5 (Ar), 125.8 (Ar), 121.3 (Ar), 120.3 (Ar), $67.3\left(\mathrm{CHCl}_{2}\right)$.

IR (neat) $\mathbf{V}_{\max } / \mathbf{c m}^{-1}: 3247,3218,1668,1544,1506,1267,1191,802$.

HRMS (ESI) m/z: [M+Na+] Calcd for $\mathrm{C}_{12} \mathrm{H}_{9} \mathrm{Cl}_{2} \mathrm{NNaO} 275.9953$; Found 275.9963.

\section{2,2-Dichloro- $N$-(2-methyl-4-fluorophenyl)acetamide 3n}<smiles>Cc1cc(F)ccc1NC(=O)C(Cl)Cl</smiles>

Amide 3n was synthesised following Procedure B, from 2-methyl-4-fluoroaniline, to yield a purple solid (190 mg, 80\%).

${ }^{1} \mathrm{H}$ NMR (400 MHz, CDCl 3$)$ ס $8.04(1 \mathrm{H}$, br. s, NH), $7.58(1 \mathrm{H}, \mathrm{m}, \mathrm{Ar}-\mathrm{H}), 6.99-6.87(2 \mathrm{H}, \mathrm{m}, \mathrm{Ar}-$ $\mathrm{H}), 6.06\left(1 \mathrm{H}, \mathrm{s}, \mathrm{CHCl}_{2}\right), 2.27\left(3 \mathrm{H}, \mathrm{s}, \mathrm{CH}_{3}\right)$.

${ }^{13}$ C NMR (101 MHz, CDCl ${ }_{3}$ ) $\delta 162.5$ (C=O), 160.9 (d, J 246 Hz, Ar), 133.7 (d, J 8.5 Hz, Ar), 129.9 (d, J 3.0 Hz, Ar), 125.6 (d, J 8.5 Hz, Ar), 117.5 (d, J 22.5 Hz, Ar), 113.7 (d, J 22.5 Hz, Ar), $66.9\left(\mathrm{CHCl}_{2}\right), 17.7\left(\mathrm{CH}_{3}\right)$.

${ }^{19} \mathrm{~F}\left(376 \mathrm{MHz}, \mathrm{CDCl}_{3}\right) \delta-115.2(\mathrm{Ar}-\mathrm{F})$

HRMS (ESI) m/z: [M+Na+] Calcd for $\mathrm{C}_{9} \mathrm{H}_{8} \mathrm{Cl}_{2} \mathrm{FNNaO}$ 257.9859; Found 257.9870.

IR (neat) $\mathbf{V}_{\max } / \mathbf{~ c m}^{-1}: 3676,3258,2988,2901,1676,1548,1497,1407,1212,1066,808,699$, 645, 505. 


\section{2,2-Dichloro- $N$-(2,6-dimethylphenyl)acetamide 30}<smiles>Cc1cccc(C)c1NC(=O)C(Cl)Cl</smiles>

Amide 30 was synthesised following Procedure B, from 2,6-dimethylaniline, to yield a yellow solid (61 mg, 26\%).

${ }^{1} \mathrm{H}$ NMR (400 MHz, $\left.\mathrm{CDCl}_{3}\right) \delta 7.72(1 \mathrm{H}, \mathrm{s}, \mathrm{NH}), 7.17(1 \mathrm{H}, \mathrm{m}, \mathrm{Ar}-\mathrm{H}), 7.12-7.08(2 \mathrm{H}, \mathrm{m}, \mathrm{Ar}-\mathrm{H})$, $6.07\left(1 \mathrm{H}, \mathrm{s}, \mathrm{CHCl}_{2}\right), 2.26(6 \mathrm{H}, \mathrm{s}, 2 \times \mathrm{Me})$.

${ }^{13} \mathrm{C}$ NMR (101 MHz, $\mathrm{CDCl}_{3}$ ) ठ 162.7 (C=O), $135.7(\mathrm{Ar}), 131.9(\mathrm{Ar}), 128.6(\mathrm{Ar}), 128.4(\mathrm{Ar}), 66.9$ $\left(\mathrm{CHCl}_{2}\right), 18.2(\mathrm{Me})$.

These data are consistent with those previously reported. ${ }^{[4]}$

\section{4-(2,2-Dichloroacetamido)benzoic acid 3p}<smiles>O=C(O)c1ccc(NC(=O)C(Cl)Cl)cc1</smiles>

Amide $3 p$ was synthesised following Procedure B, from 4-aminobenzoic acid, to yield a colourless solid (245 mg, 98\%).

${ }^{1} \mathrm{H}$ NMR (400 MHz, CD ${ }_{3}$ OD) $\delta 8.02(2 \mathrm{H}, \mathrm{d}, J 8.5 \mathrm{~Hz}, \mathrm{Ar}-\mathrm{H}), 7.72(2 \mathrm{H}, \mathrm{d}, J 8.5 \mathrm{~Hz}, \mathrm{Ar}-\mathrm{H}), 6.39$ $\left(1 \mathrm{H}, \mathrm{s}, \mathrm{CHCl}_{2}\right)$.

${ }^{13} \mathrm{C}$ NMR (101 MHz, CD $\left.\mathrm{CD}_{3} \mathrm{OD}\right) \mathrm{\delta} 169.2(\mathrm{C}=\mathrm{O}), 164.4(\mathrm{C}=\mathrm{O}), 143.1(\mathrm{Ar}), 131.9(\mathrm{Ar}), 128.1(\mathrm{Ar})$, $120.6(\mathrm{Ar}), 68.1\left(\mathrm{CHCl}_{2}\right)$.

${ }^{1} \mathbf{H}$ NMR (400 MHz, d $\mathbf{d}_{6}$-DMSO) $\delta 12.85\left(1 \mathrm{H}^{2}, \mathrm{br} \mathrm{s}, \mathrm{CO}_{2} \mathrm{H}\right), 10.93(1 \mathrm{H}, \mathrm{s}, \mathrm{NH})$ 7.97-7.93 $(2 \mathrm{H}$, m, Ar-H), 7.74-7.70 (2H, m, Ar-H), $6.61\left(1 \mathrm{H}, \mathrm{s}, \mathrm{CHCl}_{2}\right)$.

${ }^{13} \mathrm{C}$ NMR (101 MHz, $\mathbf{d}_{6}$-DMSO) $\delta 166.8(\mathrm{C}=\mathrm{O}), 162.1(\mathrm{C}=\mathrm{O}), 141.6(\mathrm{Ar}), 130.6(\mathrm{Ar}), 126.6(\mathrm{Ar})$, $119.3(\mathrm{Ar}), 67.3\left(\mathrm{CHCl}_{2}\right)$.

These data are consistent with those previously reported. ${ }^{[6]}$

2 Integrates to $<1 \mathrm{H}$ 


\section{4-(4-(2,2-dichloroacetamido)phenyl)butanoic acid 3q}<smiles>O=C(O)CCCc1ccc(NC(=O)C(Cl)Cl)cc1</smiles>

Amide 3q was synthesised following Procedure B, from 4-(4-aminophenyl)butyric acid, to yield a brown solid (277 mg, 96\%).

${ }^{1} \mathrm{H}$ NMR (400 MHz, CD $\left.{ }_{3} \mathrm{OD}\right) \delta 7.49(2 \mathrm{H}, \mathrm{d}, J 8.5 \mathrm{~Hz}, \mathrm{Ar}-\mathrm{H}), 7.17(2 \mathrm{H}, \mathrm{d}, J 8.5 \mathrm{~Hz}, \mathrm{Ar}-\mathrm{H}), 6.36$ $\left(1 \mathrm{H}, \mathrm{s}, \mathrm{CHCl}_{2}\right), 2.61\left(2 \mathrm{H}, \mathrm{m}, \mathrm{CH}_{2}\right), 2.28\left(2 \mathrm{H}, \mathrm{m}, \mathrm{CH}_{2}\right), 1.88\left(2 \mathrm{H}, \mathrm{m}, \mathrm{CH}_{2}\right)$.

${ }^{13} \mathrm{C}$ NMR (101 MHz, CD $\mathrm{CD}_{3}$ ) $\delta 177.3(\mathrm{C}=\mathrm{O}), 164.2(\mathrm{C}=\mathrm{O}), 140.1(\mathrm{Ar}), 136.4(\mathrm{Ar}), 123.0(\mathrm{Ar})$, $121.6(\mathrm{Ar}), 68.1\left(\mathrm{CHCl}_{2}\right), 35.4\left(\mathrm{CH}_{2}\right), 34.1\left(\mathrm{CH}_{2}\right), 27.7\left(\mathrm{CH}_{2}\right)$.

HRMS (ESI) m/z: [M-H] Calcd for $\mathrm{C}_{12} \mathrm{H}_{12} \mathrm{Cl}_{2} \mathrm{NO}_{3} 288.0194$; Found 288.0190.

IR (neat) $\mathbf{V}_{\max } / \mathbf{~ c m}^{-1}:$ 3673, 3320, 2972, 2900, 1707, 1413, 1084, 1065, 1046, 880.

\section{N-(4-Methoxybenzyl)-2,2-dichloroacetamide 3r}<smiles>COc1ccc(CNC(=O)C(Cl)Cl)cc1</smiles>

Amide $\mathbf{3 r}$ was synthesised following Procedure C, from 4-methoxybenzylamine, to yield a brown solid (152 mg, 61\%).

${ }^{1} \mathrm{H}$ NMR $\left(400 \mathrm{MHz}, \mathrm{CDCl}_{3}\right) \delta 7.20(2 \mathrm{H}, \mathrm{d}, J 9.0 \mathrm{~Hz}, \mathrm{Ar}-\mathrm{H}), 6.97(1 \mathrm{H}, \mathrm{s}, \mathrm{NH}), 6.87(2 \mathrm{H}, \mathrm{d}, J 9.0$ $\mathrm{Hz}, \mathrm{Ar}-\mathrm{H}), 5.94\left(1 \mathrm{H}, \mathrm{s}, \mathrm{CHCl}_{2}\right), 4.40\left(2 \mathrm{H}, \mathrm{d}, \mathrm{J} 5.5 \mathrm{~Hz}, \mathrm{CH}_{2}\right), 3.78\left(3 \mathrm{H}, \mathrm{s}, \mathrm{OCH}_{3}\right)$.

${ }^{13} \mathrm{C}$ NMR (101 MHz, $\mathrm{CDCl}_{3}$ ) ठ $164.2(\mathrm{C}=\mathrm{O}), 159.4(\mathrm{Ar}), 129.2(\mathrm{Ar}), 128.9(\mathrm{Ar}), 114.3(\mathrm{Ar}), 66.5$ $\left(\mathrm{CHCl}_{2}\right), 55.4\left(\mathrm{OCH}_{3}\right), 43.8\left(\mathrm{CH}_{2}\right)$.

HRMS (ESI) m/z: [M+Na+] Calcd for $\mathrm{C}_{10} \mathrm{H}_{11} \mathrm{Cl}_{2} \mathrm{NNaO}_{2}$ 270.0059; Found 270.0064.

IR (neat) $\mathbf{v}_{\max } / \mathbf{~ c m}^{-1}: 3682,3264,3077,2999,1664,1555,1513,1250,1031,810,655,521$. 
$\mathrm{N}$-(4-Methylbenzyl)-2,2-dichloroacetamide 3s<smiles>Cc1ccc(CNC(=O)C(Cl)Cl)cc1</smiles>

Amide 3s was synthesised following Procedure C, from 4-methylbenzylamine, to yield a colourless solid (178 mg, 77\%).

${ }^{1} \mathrm{H}$ NMR $\left(400 \mathrm{MHz}, \mathrm{CDCl}_{3}\right) \delta 7.17(4 \mathrm{H}, \mathrm{s}, \mathrm{Ar}-\mathrm{H}), 6.99(1 \mathrm{H}, \mathrm{s}, \mathrm{NH}), 5.94\left(1 \mathrm{H}, \mathrm{s}, \mathrm{CHCl}_{2}\right), 4.44$ $\left(2 \mathrm{H}, \mathrm{d}, J 5.5 \mathrm{~Hz}, \mathrm{CH}_{2}\right), 2.35\left(3 \mathrm{H}, \mathrm{s}, \mathrm{CH}_{3}\right)$.

${ }^{13} \mathrm{C}$ NMR (101 MHz, CDCl $)$ ) 164.3 (C=O), $137.8(\mathrm{Ar}), 133.7$ (Ar), 129.6 (Ar), 127.8 (Ar), 66.5 $\left(\mathrm{CHCl}_{2}\right), 44.1\left(\mathrm{CH}_{2}\right), 21.2\left(\mathrm{CH}_{3}\right)$.

HRMS (ESI) m/z: [M+Na+] Calcd for $\mathrm{C}_{10} \mathrm{H}_{11} \mathrm{Cl}_{2} \mathrm{NNaO}$ 254.0110; Found 254.0122.

IR (neat) $\mathbf{V}_{\max } / \mathbf{~ c m}^{-1}:$ 3259, 2994, 1667, 1554, 1207, 1057, 811, 802, 657, 544.

\section{$\mathrm{N}$-(4-Trifluoromethylbenzyl)-2,2-dichloroacetamide 3t}<smiles>O=C(NCc1ccc(C(F)(F)F)cc1)C(Cl)Cl</smiles>

Amide $\mathbf{3 t}$ was synthesised following Procedure $\mathbf{C}$, from 4-trifluoromethylbenzylamine, to yield a brown solid (201 mg, 70\%).

${ }^{1} \mathrm{H}$ NMR $\left(400 \mathrm{MHz}, \mathrm{CDCl}_{3}\right)$ ठ $7.58(2 \mathrm{H}, \mathrm{d}, J 8.0 \mathrm{~Hz}, \mathrm{Ar}-\mathrm{H}), 7.37(2 \mathrm{H}, \mathrm{d}, J 8.0 \mathrm{~Hz}, \mathrm{Ar}-\mathrm{H}), 7.27$ $(1 \mathrm{H}, \mathrm{s}, \mathrm{NH}), 5.98\left(1 \mathrm{H}, \mathrm{s}, \mathrm{CHCl}_{2}\right), 4.52\left(2 \mathrm{H}, \mathrm{d}, J 6.1, \mathrm{CH}_{2}\right)$.

${ }^{13} \mathrm{C}$ NMR (101 MHz, CDCl 3 ) $\delta 164.7$ (C=O), 140.1 (Ar), 130.23 (q, J 33.0 Hz, Ar), 127.8 (Ar), 125.9 (q, J $4.0 \mathrm{~Hz}, \mathrm{Ar}), 124.1$ (q, J $\left.272.0 \mathrm{~Hz}, \mathrm{CF}_{3}\right), 66.4\left(\mathrm{CHCl}_{2}\right), 43.7\left(\mathrm{CH}_{2}\right)$.

${ }^{19} \mathrm{~F}\left(376 \mathrm{MHz}, \mathrm{CDCl}_{3}\right) \delta-62.5\left(\mathrm{CF}_{3}\right)$.

HRMS (ESI) m/z: [M+Na+] Calcd for $\mathrm{C}_{10} \mathrm{H}_{8} \mathrm{Cl}_{2} \mathrm{~F}_{3} \mathrm{NNaO} 307.9827$; Found 307.9838 .

IR (neat) $\mathbf{V}_{\max } / \mathbf{~ c m}^{-1}: 3682,2986,1679,1521,1325,1264,1124,1066,738$. 
N-Benzyl-2,2-dichloroacetamide 3u<smiles>O=C(NCc1ccccc1)C(Cl)Cl</smiles>

Amide $\mathbf{3 u}$ was synthesised following Procedure $\mathbf{C}$, from benzylamine, to yield a colourless solid (167 mg, 77\%).

${ }^{1} \mathrm{H}$ NMR $\left(400 \mathrm{MHz}, \mathrm{CDCl}_{3}\right) \delta$ 7.39-7.25 (5H, m, Ar-H), $5.96\left(1 \mathrm{H}, \mathrm{s}, \mathrm{CHCl}_{2}\right), 4.47(2 \mathrm{H}, \mathrm{d}, J 6.0$ $\left.\mathrm{Hz}, \mathrm{CH}_{2}\right)$.

${ }^{13} \mathrm{C}$ NMR (101 MHz, $\mathrm{CDCl}_{3}$ ) ठ 164.3 (C=O), 136.8 (Ar), $129.0(\mathrm{Ar}), 128.0(\mathrm{Ar}), 127.7(\mathrm{Ar}), 66.5$ $\left(\mathrm{CHCl}_{2}\right), 44.3\left(\mathrm{CH}_{2}\right)$.

These data are consistent with those previously reported. ${ }^{[7]}$

\section{$N$-(4-Bromobenzyl)-2,2-dichloroacetamide 3v}<smiles>O=C(NCc1ccc(Br)cc1)C(Cl)Cl</smiles>

Amide $\mathbf{3 v}$ was synthesised following Procedure $\mathbf{C}$, from 4-bromobenzylamine. The product was additionally purified by filtering through a silica plug with pentane then dichloromethane, to yield a colourless solid (197 mg, 66\%).

${ }^{1} \mathrm{H}$ NMR (400 MHz, $\left.\mathbf{C D C l}_{3}\right) \delta 7.50-7.40(2 \mathrm{H}, \mathrm{m}, \mathrm{Ar}-\mathrm{H}), 7.16(2 \mathrm{H}, \mathrm{d}, J 8.5 \mathrm{~Hz}, \mathrm{Ar}-\mathrm{H}), 6.89$ $(1 \mathrm{H}, \mathrm{s}, \mathrm{NH}), 5.96\left(1 \mathrm{H}, \mathrm{s}, \mathrm{CHCl}_{2}\right), 4.45\left(2 \mathrm{H}, \mathrm{d}, \mathrm{J} 6.0 \mathrm{~Hz}, \mathrm{CH}_{2}\right)$.

${ }^{13} \mathrm{C}$ NMR (101 MHz, $\mathbf{C D C l}_{3}$ ) $\delta 164.4$ (C=O), 135.9 (Ar), 132.2 (Ar), 129.5 (Ar), 122.0 (Ar), 66.4 $\left(\mathrm{CHCl}_{2}\right), 43.7\left(\mathrm{CH}_{2}\right)$.

HRMS (ESI) m/z: [M+Na+] Calcd for $\mathrm{C}_{9} \mathrm{H}_{8} \mathrm{BrCl}_{2} \mathrm{NNaO}$ 317.9059; Found 317.9072.

IR (neat) $\mathbf{v}_{\max } / \mathbf{c m}^{-1}: 3679,3263,2993,1662,1538,1488,1406,1201,1070,1011,811,720$, 652. 
$N$-(4-Fluorobenzyl)-2,2-dichloroacetamide 3w<smiles>O=C(NCc1ccc(F)cc1)C(Cl)Cl</smiles>

Amide 3w was synthesised following Procedure C, from 4-fluorobenzylamine, to yield a colourless solid (232 mg, 98\%).

${ }^{1} \mathrm{H}$ NMR (400 MHz, CDCl 3$)$ ठ 7.37-7.20 (2H, m, Ar-H), $7.01(2 \mathrm{H}, \mathrm{t}, J 8.5 \mathrm{~Hz}, \mathrm{Ar}-\mathrm{H}), 5.94(1 \mathrm{H}$, $\left.\mathrm{s}, \mathrm{CHCl}_{2}\right), 4.44\left(2 \mathrm{H}, \mathrm{d}, J 5.5 \mathrm{~Hz}, \mathrm{CH}_{2}\right)$.

${ }^{13} \mathrm{C}$ NMR (101 MHz, CDCl $)$ ) 164.3 (C=O), 162.6 (d, J $\left.246.5 \mathrm{~Hz}, \mathrm{Ar}-\mathrm{F}\right), 132.7$ (d, J $3.5 \mathrm{~Hz}$, Ar), 129.6 (d, J 8.0 Hz, Ar-H), 115.9 (d, J21.5, Ar-H), $66.5\left(\mathrm{CHCl}_{2}\right), 43.6\left(\mathrm{CH}_{2}\right)$.

${ }^{19} \mathrm{~F}\left(376 \mathrm{MHz}, \mathrm{CDCl}_{3}\right) \delta-114.0(\mathrm{C}-\mathrm{F})$

HRMS (ESI) m/z: [M+Na+] Calcd for $\mathrm{C}_{9} \mathrm{H}_{8} \mathrm{Cl}_{2} \mathrm{FNNaO}$ 257.9859; Found 257.9867.

IR (neat) $\mathbf{v}_{\max } / \mathbf{c m}^{-1}: 3272,3010,1661,1547,1509,1218,1158,810,784,646$.

\section{2,2-Dichloro- $N$-(3,5-dimethoxybenzyl)acetamide $3 x$}<smiles>COc1cc(CNC(=O)C(Cl)Cl)cc(OC)c1</smiles>

Amide 3x was synthesised following Procedure $\mathbf{C}$, from 3,5-dimethoxybenzylamine, to yield a colourless solid (226 mg, 81\%).

${ }^{1} \mathrm{H}$ NMR (400 MHz, CDCl $\left.{ }_{3}\right) \delta 6.93(1 \mathrm{H}, \mathrm{br} \mathrm{s}, \mathrm{NH}), 6.42-6.40(2 \mathrm{H}, \mathrm{m}, \mathrm{Ar}-\mathrm{H}), 6.38(1 \mathrm{H}, \mathrm{t}, J 2.5$ $\mathrm{Hz}, \mathrm{Ar}-\mathrm{H}), 5.96\left(1 \mathrm{H}, \mathrm{s}, \mathrm{CHCl}_{2}\right), 4.42\left(2 \mathrm{H}, \mathrm{d}, J 6.0 \mathrm{~Hz}, \mathrm{CH}_{2}\right), 3.76(6 \mathrm{H}, \mathrm{s}, 2 \times \mathrm{OMe})$.

${ }^{13} \mathrm{C}$ NMR (101 MHz, $\mathrm{CDCl}_{3}$ ) $\delta 164.3(\mathrm{C}=\mathrm{O}), 161.3(\mathrm{Ar}), 139.2(\mathrm{Ar}), 105.6(\mathrm{Ar}), 99.8(\mathrm{Ar}), 66.5$ $\left(\mathrm{CHCl}_{2}\right), 55.5(\mathrm{OMe}), 44.3\left(\mathrm{CH}_{2}\right)$.

HRMS (ESI) m/z: [M+Na+] Calcd for $\mathrm{C}_{11} \mathrm{H}_{13} \mathrm{Cl}_{2} \mathrm{NNaO}_{3} 300.0165$; Found 300.0173 .

IR (neat) $\mathbf{V}_{\max } / \mathbf{~ c m}^{-1}:$ : 3678, 3259, 3008, 2941, 2645, 1660, 1593, 1547, 1460, 1330, 1196, 1153, 1070, 932, 811, 769, 655 


\section{2,2-dichloro- $N$-(naphthalen-1-ylmethyl)acetamide 3y}<smiles>O=C(NCc1cccc2ccccc12)C(Cl)Cl</smiles>

Amide 3y was synthesised following Procedure $\mathbf{C}$, from naphthylmethylamine and was additionally purified by filtering through a silica plug with $20 \%$ ethyl acetate in hexane, to yield a colourless solid (146 mg, 55\%).

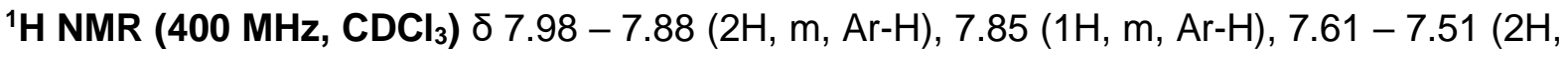
m, Ar-H), 7.46-7.44 (2H, m, Ar-H), $6.84(1 \mathrm{H}, \mathrm{s}, \mathrm{NH}), 5.97\left(1 \mathrm{H}, \mathrm{s}, \mathrm{CHCl}_{2}\right), 4.93(2 \mathrm{H}, \mathrm{d}, J 5.5 \mathrm{~Hz}$, $\mathrm{CH}_{2}$ ).

${ }^{13} \mathrm{C}$ NMR (101 MHz, CDCl 3 ) ס $164.0(\mathrm{C}=\mathrm{O}), 134.0(\mathrm{Ar}), 132.0(\mathrm{Ar}), 131.4(\mathrm{Ar}), 129.2(\mathrm{Ar}), 129.0$ (Ar), $127.0(\mathrm{Ar}), 126.9(\mathrm{Ar}), 126.3(\mathrm{Ar}), 125.5(\mathrm{Ar}), 123.2(\mathrm{Ar}), 66.5\left(\mathrm{CHCl}_{2}\right), 42.6\left(\mathrm{CH}_{2}\right)$.

HRMS (ESI) m/z: [M+Na+] Calcd for $\mathrm{C}_{13} \mathrm{H}_{11} \mathrm{Cl}_{2} \mathrm{NNaO} 290.0110$; Found 290.0116.

IR (neat) $\mathbf{V}_{\max } / \mathbf{~ c m}^{-1}: 3672,3271,3001,1663,1547,1205,1059,813,776,659$.

\section{2,2-Dichloro- $N$-cyclobutylacetamide $3 z$}<smiles>O=C(NC1CCC1)C(Cl)Cl</smiles>

Amide $\mathbf{3 z}$ was synthesised following Procedure $\mathbf{C}$, from cyclobutylamine, to yield a brown solid (102 mg, 56\%).

${ }^{1} \mathrm{H}$ NMR (400 MHz, CDCl $)$ ס $6.76(1 \mathrm{H}, \mathrm{s}, \mathrm{NH}), 5.90\left(1 \mathrm{H}, \mathrm{s}, \mathrm{CHCl}_{2}\right), 4.35(1 \mathrm{H}, \mathrm{m}, \mathrm{CH}), 2.43-$ $2.29(2 \mathrm{H}, \mathrm{m}, 2 \times \mathrm{CH}), 2.05-1.88(2 \mathrm{H}, \mathrm{m}, 2 \times \mathrm{CH}), 1.78-1.65\left(2 \mathrm{H}, \mathrm{m}, \mathrm{CH}_{2}\right)$.

${ }^{13} \mathrm{C}$ NMR (101 MHz, $\left.\mathbf{C D C l}_{3}\right) \delta 163.3(\mathrm{C}=\mathrm{O}), 66.5\left(\mathrm{CHCl}_{2}\right), 45.6(\mathrm{CH}), 30.7\left(2 \times \mathrm{CH}_{2}\right), 15.2$ $\left(\mathrm{CH}_{2}\right)$.

HRMS (ESI) m/z: [M+Na+] Calcd for $\mathrm{C}_{6} \mathrm{H}_{9} \mathrm{Cl}_{2} \mathrm{NNaO}$ 203.9953; Found 203.9955.

IR (neat) $\mathbf{V}_{\max } / \mathbf{c m}^{-1}: 3256,3075,2971,1661,1556,1339,1245,1218,908,810,649$. 


\section{2,2-Dichloro-N-cyclopentylacetamide 3aa}<smiles>O=C(NC1CCCC1)C(Cl)Cl</smiles>

Amide 3aa was synthesised following Procedure $\mathbf{C}$, from cyclopentylamine, to yield a colourless solid (122 mg, 62\%).

${ }^{1} \mathrm{H}$ NMR (400 MHz, $\left.\mathrm{CDCl}_{3}\right) \delta 6.53\left(1 \mathrm{H}\right.$, br. s, NH), $5.91\left(1 \mathrm{H}, \mathrm{s}, \mathrm{CHCl}_{2}\right), 4.17(1 \mathrm{H}, \mathrm{m}, \mathrm{C}-\mathrm{H}), 2.09$ $-1.93(2 \mathrm{H}, \mathrm{m}, 2 \times \mathrm{CH}), 1.79-1.60\left(4 \mathrm{H}, \mathrm{m}, 2 \times \mathrm{CH}_{2}\right), 1.54-1.39(2 \mathrm{H}, \mathrm{m}, 2 \times \mathrm{CH})$.

${ }^{13} \mathrm{C}$ NMR (101 MHz, $\left.\mathrm{CDCl}_{3}\right) \delta 163.8(\mathrm{C}=\mathrm{O}), 66.6\left(\mathrm{CHCl}_{2}\right), 55.3(\mathrm{CH}), 32.8\left(\mathrm{CH}_{2}\right), 23.8\left(\mathrm{CH}_{2}\right)$. HRMS (ESI) m/z: [M+Na+] Calcd for $\mathrm{C}_{7} \mathrm{H}_{11} \mathrm{Cl}_{2} \mathrm{NNaO} 218.0110$; Found 218.0113.

IR (neat) $\mathbf{v}_{\max } / \mathbf{~ c m}^{-1}: 3679,3262,2930,2860,1683,1664,1557,1526,1448,1354,1208$, $1053,812,658$.

\section{2,2-Dichloro- $N$-cyclohexylacetamide 3ab}<smiles>O=C(NC1CCCCC1)C(Cl)Cl</smiles>

Amide 3ab was synthesised following Procedure $\mathbf{C}$, from cyclohexylamine, to yield a colourless solid (109 mg, 52\%).

${ }^{1} \mathrm{H}$ NMR (400 MHz, $\left.\mathrm{CDCl}_{3}\right) \delta 6.52\left(1 \mathrm{H}\right.$, br. s, NH), $5.91\left(1 \mathrm{H}, \mathrm{s}, \mathrm{CHCl}_{2}\right), 3.73(1 \mathrm{H}, \mathrm{m}, \mathrm{CH}), 1.96-$ $1.88(2 \mathrm{H}, \mathrm{m}, 2 \times \mathrm{CHH}) .1 .75-1.66(2 \mathrm{H}, \mathrm{m}, 2 \times \mathrm{CH}), 1.61(1 \mathrm{H}, \mathrm{m}, \mathrm{CHH}), 1.41-1.29(2 \mathrm{H}, \mathrm{m}, 2$ $\times \mathrm{CHH}), 1.27-1.14(3 \mathrm{H}, \mathrm{m}, 3 \times \mathrm{CH})$.

${ }^{13} \mathrm{C}$ NMR (101 MHz, CDCl 3$) \delta 163.3(\mathrm{C}=\mathrm{O}), 66.7\left(\mathrm{CHCl}_{2}\right), 49.4(\mathrm{CH}), 32.5\left(\mathrm{CH}_{2}\right), 25.4\left(\mathrm{CH}_{2}\right)$, $24.7\left(\mathrm{CH}_{2}\right)$.

HRMS (ESI) m/z: [M+Na+] Calcd for $\mathrm{C}_{8} \mathrm{H}_{13} \mathrm{Cl}_{2} \mathrm{NNaO}$ 232.0266; Found 232.0269.

These data are consistent with those previously reported. ${ }^{[8]}$ 


\section{2,2-Dichloro-N-cycloheptylacetamide 3ac}<smiles>O=C(NC1CCCCCC1)C(Cl)Cl</smiles>

Amide 3ac was synthesised following Procedure $\mathbf{C}$, from cycloheptylamine, to yield a brown solid (122 mg, 55\%).

${ }^{1} \mathrm{H}$ NMR $\left(400 \mathrm{MHz}, \mathrm{CDCl}_{3}\right) \delta 6.56(1 \mathrm{H}, \mathrm{s}, \mathrm{NH}), 5.91\left(1 \mathrm{H}, \mathrm{s}, \mathrm{CHCl}_{2}\right), 3.91(1 \mathrm{H}, \mathrm{m}, \mathrm{CH}), 1.96-$ $1.87(2 \mathrm{H}, \mathrm{m}, 2 \times \mathrm{CH}), 1.79-0.87\left(10 \mathrm{H}, \mathrm{m}, 2 \times \mathrm{CH}, 4 \times \mathrm{CH}_{2}\right)$.

${ }^{13} \mathrm{C}$ NMR (101 MHz, CDCl $\left.)_{3}\right) 163.0(\mathrm{C}=\mathrm{O}), 66.7\left(\mathrm{CHCl}_{2}\right), 51.6(\mathrm{CH}), 34.5\left(\mathrm{CH}_{2}\right), 27.9\left(\mathrm{CH}_{2}\right)$, $24.0\left(\mathrm{CH}_{2}\right)$.

HRMS (ESI) m/z: [M+Na+] Calcd for $\mathrm{C}_{9} \mathrm{H}_{15} \mathrm{Cl}_{2} \mathrm{NNaO} 246.0423$; Found 246.0418.

IR (neat) $\mathbf{v}_{\max } / \mathbf{~ c m}^{-1}: 3668,3326,3285,2961,2874,1686,1665,1551,1532,1453,1210$, $811,642$.

\section{2,2-Dichloro- $N$-(pentan-3-yl)acetamide 3ad}<smiles>CCC(CC)NC(=O)C(Cl)Cl</smiles>

Amide $\mathbf{3 a d}$ was synthesised following Procedure $\mathbf{C}$, from 3-aminopentane, to yield an orange solid (85 mg, 43\%).

${ }^{1} \mathrm{H}$ NMR $\left(400 \mathrm{MHz}, \mathrm{CDCl}_{3}\right) \delta 6.29(1 \mathrm{H}, \mathrm{s}, \mathrm{NH}), 5.94\left(1 \mathrm{H}, \mathrm{s}, \mathrm{CHCl}_{2}\right), 3.81-3.67(1 \mathrm{H}, \mathrm{m}, \mathrm{CH})$, 1.68-1.54 (2H, m, $\left.\mathrm{CH}_{2}\right), 1.52-1.35\left(2 \mathrm{H}, \mathrm{m}, \mathrm{CH}_{2}\right), 0.94-0.87\left(3 \mathrm{H}, \mathrm{m}, \mathrm{CH}_{3}\right)$.

${ }^{13} \mathrm{C}$ NMR (101 MHz, CDCl 3$) \delta 164.3(\mathrm{C}=\mathrm{O}), 66.9\left(\mathrm{CHCl}_{2}\right), 53.4(\mathrm{CH}), 27.4\left(\mathrm{CH}_{2}\right), 27.3\left(\mathrm{CH}_{2}\right)$, $10.2\left(\mathrm{CH}_{3}\right)$.

HRMS (ESI) m/z: [M+Na+] Calcd for $\mathrm{C}_{7} \mathrm{H}_{13} \mathrm{Cl}_{2} \mathrm{NNaO}$ 220.02664; Found 220.0269.

IR (neat) $\mathbf{V}_{\max } / \mathbf{~ c m}^{-1}:$ 3679, 3273, 2970, 2901, 1670, 1566, 1406, 1394, 1225, 1056, 813, 663 


\section{2,2-Dichloro- $N$-butylacetamide 3ae}<smiles>CCCCNC(=O)C(Cl)Cl</smiles>

Amide 3ae was synthesised following Procedure $\mathbf{C}$, from 1-butylamine, to yield an orange solid (95 mg, 52\%).

${ }^{1} \mathrm{H}$ NMR $\left(400 \mathrm{MHz}, \mathrm{CDCl}_{3}\right) \delta 6.74(1 \mathrm{H}, \mathrm{s}, \mathrm{NH}), 5.94\left(1 \mathrm{H}, \mathrm{s}, \mathrm{CHCl}_{2}\right), 3.31(2 \mathrm{H}, \mathrm{td}, J 7.0,6.0 \mathrm{~Hz}$, $\left.\mathrm{NHCH}_{2}\right), 1.62-1.48\left(2 \mathrm{H}, \mathrm{m}, \mathrm{CH}_{2}\right), 1.42-1.29\left(2 \mathrm{H}, \mathrm{m}, \mathrm{CH}_{2}\right), 0.92\left(3 \mathrm{H}, \mathrm{t}, J 7.5 \mathrm{~Hz}, \mathrm{CH}_{3}\right)$.

${ }^{13} \mathrm{C}$ NMR (101 MHz, $\left.\mathrm{CDCl}_{3}\right) \delta 164.3(\mathrm{C}=\mathrm{O}), 66.6\left(\mathrm{CHCl}_{2}\right), 40.2\left(\mathrm{NHCH}_{2}\right), 31.2\left(\mathrm{CH}_{2}\right), 20.0$ $\left(\mathrm{CH}_{2}\right), 13.7\left(\mathrm{CH}_{3}\right)$.

HRMS (ESI) m/z: [M+Na+] Calcd for $\mathrm{C}_{6} \mathrm{H}_{11} \mathrm{Cl}_{2} \mathrm{NNaO} 206.0110$; Found 206.0115.

IR (neat) $\mathbf{V}_{\max } / \mathbf{~ c m}^{-1}:$ 3669, 3253, 3085, 2960, 2934, 2873, 1666, 1561, 1461, 1346, 1211 , $814,735,661$

\section{2,2-Dichloro- $N$-octylacetamide 3af}<smiles>CCCCCCCCNC(=O)C(Cl)Cl</smiles>

Amide 3af was synthesised following Procedure $\mathbf{C}$, from 1-octylamine, to yield an orange solid (155 mg, 65\%).

${ }^{1} \mathrm{H}$ NMR $\left(400 \mathrm{MHz}, \mathrm{CDCl}_{3}\right) \delta 6.85\left(1 \mathrm{H}\right.$, br. s, NH), $5.92\left(1 \mathrm{H}, \mathrm{s}, \mathrm{CHCl}_{2}\right), 3.31(2 \mathrm{H}, \mathrm{q}, J 7.0 \mathrm{~Hz}$, $\left.\mathrm{CH}_{2}\right), 1.60-1.52\left(2 \mathrm{H}, \mathrm{m}, \mathrm{CH}_{2}\right), 1.35-1.21\left(10 \mathrm{H}, \mathrm{m}, 5 \times \mathrm{CH}_{2}\right), 0.87\left(3 \mathrm{H}, \mathrm{t}, J 6.5 \mathrm{~Hz}, \mathrm{CH}_{3}\right)$.

${ }^{13} \mathrm{C} \mathrm{NMR}\left(101 \mathrm{MHz}, \mathrm{CDCl}_{3}\right) \delta 164.2(\mathrm{C}=\mathrm{O}), 66.7\left(\mathrm{CHCl}_{2}\right), 40.5\left(\mathrm{CH}_{2}\right), 31.9\left(\mathrm{CH}_{2}\right), 29.3\left(\mathrm{CH}_{2}\right)$, $29.2\left(\mathrm{CH}_{2}\right), 29.2\left(\mathrm{CH}_{2}\right), 26.8\left(\mathrm{CH}_{2}\right), 22.7\left(\mathrm{CH}_{2}\right), 14.2\left(\mathrm{CH}_{3}\right)$.

HRMS (ESI) m/z: [M+Na+] Calcd for $\mathrm{C}_{10} \mathrm{H}_{19} \mathrm{Cl}_{2} \mathrm{NNaO}$ 262.0736; Found 262.0746.

IR (neat) $\mathbf{V}_{\max } / \mathbf{c m}^{-1}: 3264,2923,2854,1668,1561,1549,1206,813,658,404$. 


\section{2,2-Dichloro-N-(2-phenylethyl)acetamide 3ag}<smiles>O=C(NCCc1ccccc1)C(Cl)Cl</smiles>

Amide 3ag was synthesised following Procedure C, from 2-phenyl-1-ethylamine, to yield an orange solid (151 mg, 65\%).

${ }^{1} \mathrm{H}$ NMR (400 MHz, $\left.\mathrm{CDCl}_{3}\right)$ ठ 7.38-7.28 (2H, m, ArH), $7.26-7.17(3 \mathrm{H}, \mathrm{m}, \mathrm{ArH}), 6.77(1 \mathrm{H}, \mathrm{s}$, $\mathrm{NH}), 5.90\left(1 \mathrm{H}, \mathrm{s}, \mathrm{CHCl}_{2}\right), 3.57\left(2 \mathrm{H}, \mathrm{m}, \mathrm{NHCH}_{2}\right), 2.88\left(2 \mathrm{H}, \mathrm{t}, J 7.0 \mathrm{~Hz}, \mathrm{PhCH}_{2}\right)$.

${ }^{13} \mathrm{C}$ NMR (101 MHz, $\mathrm{CDCl}_{3}$ ) ठ $164.2(\mathrm{C}=\mathrm{O}), 138.2(\mathrm{Ar}), 128.9(\mathrm{Ar}), 128.8(\mathrm{Ar}), 126.8(\mathrm{Ar}), 66.5$ $\left(\mathrm{CHCl}_{2}\right), 41.6\left(\mathrm{NHCH}_{2}\right), 35.2\left(\mathrm{PHCH}_{2}\right)$.

HRMS (ESI) m/z: [M+Na+] Calcd for ${ }_{10} \mathrm{H}_{11} \mathrm{Cl}_{2} \mathrm{NNaO} 254.0110$; Found 254.0113.

IR (neat) $\mathbf{v}_{\max } / \mathbf{~ c m}^{-1}: 3676,3228,3080,2988,2952,1668,1579,1493,1440,1344,1199$, 1039, 909, 810, 702, 657, 495, 457.

These data are consistent with those previously reported. ${ }^{[9]}$

\section{2,2-Dichloro-N-(3-phenylpropyl)acetamide 3ah}<smiles>O=C(NCCCc1ccccc1)C(Cl)Cl</smiles>

Amide 3ah was synthesised following Procedure C, from 3-phenyl-1-propylamine, to yield a colourless solid (202 mg, 82\%).

${ }^{1} \mathrm{H}$ NMR (400 MHz, CDCl $)$ ס $7.35-7.26(2 \mathrm{H}, \mathrm{m}, \mathrm{Ar}-\mathrm{H}), 7.21-7.15(3 \mathrm{H}, \mathrm{m}, \mathrm{Ar}-\mathrm{H}), 6.97(1 \mathrm{H}$, s, $\mathrm{NH}), 5.98\left(1 \mathrm{H}, \mathrm{s}, \mathrm{CHCl}_{2}\right), 3.36\left(2 \mathrm{H}, \mathrm{td}, J 7.0,6.0 \mathrm{~Hz}, \mathrm{CH}_{2}\right), 2.73-2.64\left(2 \mathrm{H}, \mathrm{m}, \mathrm{CH}_{2}\right), 1.93$ $\left(2 \mathrm{H}, \mathrm{tt}, J 7.5,6.5 \mathrm{~Hz}, \mathrm{CH}_{2}\right)$.

${ }^{13} \mathrm{C}$ NMR (101 MHz, $\mathrm{CDCl}_{3}$ ) $\delta 164.4(\mathrm{C}=\mathrm{O}), 141.0(\mathrm{Ar}), 128.6(\mathrm{Ar}), 128.4(\mathrm{Ar}), 126.2(\mathrm{Ar}), 66.6$ $\left(\mathrm{CHCl}_{2}\right), 40.0\left(\mathrm{CH}_{2}\right), 33.1\left(\mathrm{CH}_{2}\right), 30.5\left(\mathrm{CH}_{2}\right)$.

HRMS (ESI) m/z: [M+Na+] Calcd for $\mathrm{C}_{11} \mathrm{H}_{13} \mathrm{Cl}_{2} \mathrm{NNaO}$ 268.0266; Found 268.0269.

IR (neat) $\mathbf{v}_{\max } / \mathbf{~ c m}^{-1}:$ 3264, 3085, 2936, 1666, 1558, 1454, 1216, 813, 746, 699, 661. 
Ethyl (2,2-dichloroacetyl)-L-phenylalaninate 3ai<smiles>CCOC(=O)[C@H](Cc1ccccc1)NC(=O)C(Cl)Cl</smiles>

Amide 3ai was synthesised following Procedure C, from L-phenylalanine ethyl ester, to yield a colourless solid (266 mg, 88\%).

${ }^{1} \mathrm{H}$ NMR (400 MHz, $\left.\mathrm{CDCl}_{3}\right) \delta 7.32-7.19(3 \mathrm{H}, \mathrm{m}, \mathrm{Ar}-\mathrm{H}), 7.16-7.07(3 \mathrm{H}, \mathrm{m}, 2 \times \mathrm{Ar}-\mathrm{H}, \mathrm{NH})$, $5.92\left(1 \mathrm{H}, \mathrm{s}, \mathrm{CHCl}_{2}\right), 4.80(1 \mathrm{H}, \mathrm{dt}, J 7.8,5.8, \mathrm{C} \underline{\mathrm{HNH}}), 4.19\left(2 \mathrm{H}, \mathrm{q}, J 7.2, \mathrm{OCH}_{2}\right), 3.19(1 \mathrm{H}, \mathrm{dd}$, J 13.9, 5.8, $\mathrm{PhCHH}$ ), $3.13(1 \mathrm{H}, \mathrm{dd}, \mathrm{J} 13.9,5.8, \mathrm{PhCHH}), 1.25\left(3 \mathrm{H}, \mathrm{t}, \mathrm{J} 7.0 \mathrm{~Hz}, \mathrm{CH}_{3}\right)$.

${ }^{13} \mathrm{C}$ NMR (101 MHz, CDCl $)$ ) 170.5 (OOt), 163.6 (C=O), 135.2 (Ar), 129.4 (Ar), 128.6 (Ar), $127.3(\mathrm{Ar}), 66.1\left(\mathrm{CHCl}_{2}\right), 61.9\left(\mathrm{OCH}_{2}\right), 53.9(\mathrm{CH}), 37.4\left(\mathrm{CH}_{2}\right), 14.1\left(\mathrm{CH}_{3}\right)$.

HRMS (ESI) m/z: [M+Na+] Calcd for $\mathrm{C}_{13} \mathrm{H}_{15} \mathrm{Cl}_{2} \mathrm{NNaO}_{3} 326.0321$; Found 326.0331 .

IR (neat) $\mathbf{V}_{\max } / \mathbf{~ c m}^{-1}: 3307,3006,1732,1668,1535,1375,1229,1202,813,699,626$

Optical Rotation: $[\alpha]_{\mathrm{D}}^{22}+52\left(c 1.0, \mathrm{CHCl}_{3}\right)$

\section{Methyl (S)-3-(4-(tert-butoxy)phenyl)-2-(2,2-dichloroacetamido)propanoate 3aj}<smiles>CCOc1ccc(C[C@H](NC(=O)C(Cl)C(Cl)Cl)C(=O)OC)cc1</smiles>

Amide 3aj was synthesised following Procedure C, from O-tert-Butyl-L-tyrosine methyl ester, to yield a yellow solid (272 $\mathrm{mg}, 75 \%)$.

${ }^{1} \mathrm{H}$ NMR (400 MHz, $\left.\mathrm{CDCl}_{3}\right) \delta 7.05-6.95(3 \mathrm{H}, \mathrm{m}, 2 \times \mathrm{Ar}-\mathrm{H}, \mathrm{NH}), 6.93-6.85(2 \mathrm{H}, \mathrm{m}, \mathrm{Ar}-\mathrm{H})$, $5.91\left(1 \mathrm{H}, \mathrm{s}, \mathrm{CHCl}_{2}\right), 4.77(1 \mathrm{H}, \mathrm{dt}, J 8.0,6.0 \mathrm{~Hz}, \mathrm{CHNH}), 3.71\left(3 \mathrm{H}, \mathrm{s}, \mathrm{OCH}_{3}\right), 3.15(1 \mathrm{H}, \mathrm{dd}, J$ 14.0, $6.0 \mathrm{~Hz}, \mathrm{CHH}), 3.09(1 \mathrm{H}, \mathrm{dd}, J$ 14.0, $6.0 \mathrm{~Hz}, \mathrm{CH} H) 1.30\left(9 \mathrm{H}, \mathrm{s}, \mathrm{OC}\left(\mathrm{CH}_{3}\right)\right)$.

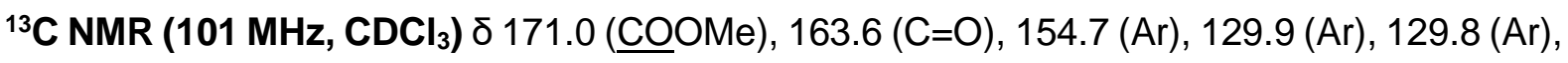
$\left.124.4(\mathrm{Ar}), 78.5(\mathrm{O} \underline{\mathrm{CMe}})_{3}\right), 66.1\left(\mathrm{CHCl}_{2}\right), 53.9(\mathrm{CHNH}), 52.6\left(\mathrm{OCH}_{3}\right), 36.9\left(\mathrm{CH}_{2}\right), 28.9\left(\mathrm{C}\left(\underline{\mathrm{C}} \mathrm{H}_{3}\right)\right)$.

HRMS (ESI) m/z: [M+Na+] Calcd for $\mathrm{C}_{16} \mathrm{H}_{21} \mathrm{Cl}_{2} \mathrm{NNaO}_{4}$ ) 384.0740; Found 384.0757. 
IR (neat) $\mathbf{V}_{\max } / \mathbf{~ c m}^{-1}: 3302,2978,2157,1741,1505,1364,1161,909,734,456$

Optical Rotation: $[\alpha]_{\mathrm{D}}^{22}+56\left(c 1.0, \mathrm{CHCl}_{3}\right)$

\section{2,2-dichloro-1-(piperidin-1-yl)ethan-1-one 3ak}<smiles>O=C(C(Cl)Cl)N1CCCCC1</smiles>

Amide 3ak was synthesised from piperidine following Procedure $\mathbf{C}$, stirring the reaction mixture for an additional $72 \mathrm{~h}$ at room temperature, to yield a waxy yellow solid (74 mg, 37\%).

${ }^{1} \mathrm{H}$ NMR (400 MHz, $\left.\mathrm{CDCl}_{3}\right) \delta 6.22\left(1 \mathrm{H}, \mathrm{s}, \mathrm{CHCl}_{2}\right), 3.77-3.37\left(4 \mathrm{H}, \mathrm{m}, \mathrm{CH}_{2} \mathrm{NCH}_{2}\right), 1.72-1.55$ $\left(6 \mathrm{H}, \mathrm{m}, 3 \times \mathrm{CH}_{2}\right)$.

${ }^{13} \mathrm{C}$ NMR (101 MHz, $\left.\mathrm{CDCl}_{3}\right) \delta 162.0(\mathrm{C}=\mathrm{O}), 65.9\left(\mathrm{CHCl}_{2}\right), 47.6\left(\mathrm{CH}_{2}\right), 44.6\left(\mathrm{CH}_{2}\right), 26.0\left(\mathrm{CH}_{2}\right)$, $25.5\left(\mathrm{CH}_{2}\right), 24.2\left(\mathrm{CH}_{2}\right)$.

These data are consistent with those previously reported. ${ }^{[10]}$ 


\section{Preparation of 2,2-dichloro- $N$-(4-fluorophenyl)acetamide $3 a$ on 5 mmol scale}<smiles>Nc1ccc(F)cc1</smiles><smiles>CC1(C)OC(=O)C(Cl)(Cl)C(=O)O1</smiles>

1.2 equiv.

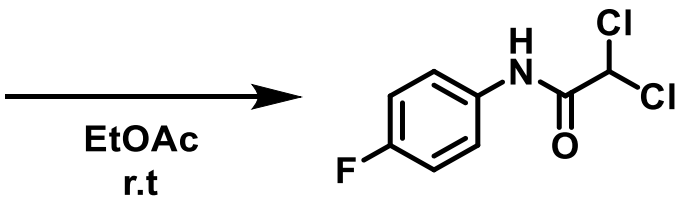

$16 \mathrm{~h}$

To a $25 \mathrm{~mL}$ round-bottomed flask equipped with a magnetic stirrer bar was added dichloromeldrum's acid (1.28 g, $6.00 \mathrm{mmol}, 1.20$ equiv.) and ethyl acetate ( $12.5 \mathrm{~mL})$, followed by addition of 4 -fluoroaniline (556 mg, $474 \mu \mathrm{L}, 1.00$ equiv.) The reaction mixture was stirred at room temperature for $16 \mathrm{~h}$ and then hydrochloric acid $(2.0 \mathrm{M}, 30 \mathrm{~mL})$ and diethyl ether (30 $\mathrm{mL}$ ) were added. The layers were separated, and the organic layer washed with hydrochloric acid $(2.0 \mathrm{M}, 30 \mathrm{~mL})$ then saturated aqueous $\mathrm{NaHCO}_{3}(3 \times 30 \mathrm{~mL})$. The organic layer was dried over $\mathrm{MgSO}_{4}$, filtered, and concentrated in vacuo to yield the amide 3a as a brown solid (1.110 g, 99\%). NMR spectra as previously reported for $\mathbf{3 a}$.

\section{Investigations into the conversion of $\mathbf{N}$-(4-methylbenzyl)-2,2- dichloroacetamide to $N$-(4-methylbenzyl)-2,2,2-trichloroacetamide}<smiles>Cc1ccc(CNC(=O)C(Cl)Cl)cc1</smiles><smiles>CC1(C)OC(=O)C(Cl)(Cl)C(=O)O1</smiles>

1.2 equiv.

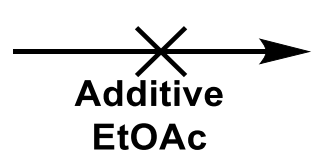

r.t, $16 \mathrm{~h}$<smiles>Cc1ccc(CNC(=O)C(Cl)(Cl)Cl)cc1</smiles>

O

To a $2 \mathrm{~mL}$ vial equipped with a magnetic stirrer bar was added N-(4-methylbenzyl)-2,2dichloroacetamide $3 \mathrm{~s}$ (20mg, $0.09 \mathrm{mmol})$, ethyl acetate $(0.5 \mathrm{~mL})$, an additive (1.5 equivalents, if used), and dichloromeldrum's acid 2 (22 $\mathrm{mg}, 0.10 \mathrm{mmol}, 1.2$ equiv.). The reaction mixture was stirred at room temperature for $16 \mathrm{~h}$ and then hydrochloric acid (2.0 M, $5 \mathrm{~mL})$ and diethyl ether $(5 \mathrm{~mL})$ were added. The layers were separated, and the organic layer washed with hydrochloric acid $(2.0 \mathrm{M}, 5 \mathrm{~mL})$ then saturated aqueous $\mathrm{NaHCO}_{3}(3 \times 5 \mathrm{~mL})$. The organic layer was dried over $\mathrm{MgSO}_{4}$, filtered, and concentrated in vacuo.

Reaction was performed in the absence of an additive, with triethylamine $(18 \mu \mathrm{L}, 0.13 \mathrm{mmol}$, 1.5 equiv.), and with formic acid ( $5 \mu \mathrm{L}, 0.13 \mathrm{mmol}, 1.5$ equiv.). In all cases only starting material 3s was returned. 


\section{Preparation of 2,2-dichloro- $N$-phenylacetamide by amidation with dichloroacetic acid}

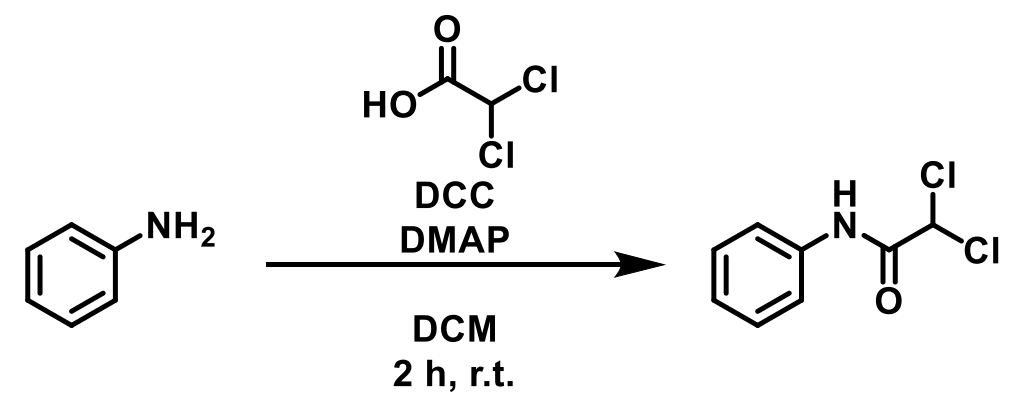

Following a literature procedure, ${ }^{[11]}$ to a solution of aniline $(93 \mathrm{mg}, 79 \mu \mathrm{L}, 1.0 \mathrm{mmol}$ ) in dry dichloromethane $(20 \mathrm{~mL})$ was added DMAP $(20 \mathrm{mg}, 0.16 \mathrm{mmol}, 0.16$ equiv.) and dichloroacetic acid (155 mg, $99.0 \mu \mathrm{L}, 1.20 \mathrm{mmol}, 1.20$ equiv.). After stirring at room temperature for $0.5 \mathrm{~h}$, dicyclohexylcarbodiimide (DCC) (248 mg, $1.20 \mathrm{mmol}, 1.20$ equiv.) was added. The reaction was monitored by TLC, and after $2 \mathrm{~h}$ the reaction mixture was concentrated in vacuo and purified by silica gel flash column chromatography $(5-15 \%$ EtOAc:Pentane) to yield 2,2-dichloro- $N$-phenylacetamide $\mathbf{3 d}$ as a colourless solid (194 $\mathrm{mg}$, 95\%).

NMR spectra as reported for $\mathbf{3 d}$. 


\section{Analysis of Enantiomeric Excess of 3ai}<smiles>NC(Cc1ccccc1)C(=O)O</smiles>

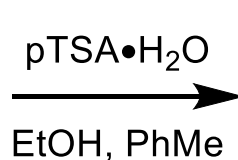

$\mathrm{EtOH}, \mathrm{PhMe}$<smiles>CCOC(=O)C(N)Cc1ccccc1</smiles>

rac-1ai<smiles>CCOC(=O)C(Cc1ccccc1)NC(=O)C(Cl)Cl</smiles>

rac-3ai

Following a literature procedure, ${ }^{[12]}$ to a suspension of DL-phenylalanine $(2.00 \mathrm{~g}, 12.1 \mathrm{mmol})$ in toluene $(20 \mathrm{~mL})$ was added $p$-toluenesulfonic acid monohydrate $(2.76 \mathrm{~g}, 14.5 \mathrm{mmol}$, 1.20 equiv.) and ethanol (670 mg, $850 \mu \mathrm{L}, 14.5 \mathrm{mmol}, 1.20$ equiv.). The suspension was heated to $80^{\circ} \mathrm{C}$ for $16 \mathrm{~h}$, then allowed to cool to room temperature. The crude $p$ TSA salt was obtained by concentrating the reaction mixture in vacuo, which was then dissolved in saturated aqueous sodium carbonate solution $(30 \mathrm{~mL})$ and dichloromethane $(30 \mathrm{~mL})$. The layers were separated, and the aqueous layer further extracted with dichloromethane $(2 \times 30 \mathrm{~mL})$. The combined organic layers were then washed with water $(3 \times 30 \mathrm{~mL})$, dried over $\mathrm{MgSO}_{4}$, and concentrated in vacuo to yield DL-phenylalanine ethyl ester rac-1ai as a pale yellow oil (1.94 g, 83\%).

${ }^{1} \mathrm{H}$ NMR (400 MHz, CDCl $\left.{ }_{3}\right) \delta 7.30-7.23(2 \mathrm{H}, \mathrm{m}, \mathrm{Ar}-\mathrm{H}), 7.23-7.14(3 \mathrm{H}, \mathrm{m}, \mathrm{Ar}-\mathrm{H}), 4.12(2 \mathrm{H}$, qd, J 7.0, $1.5 \mathrm{~Hz}, \mathrm{OCH}_{2}$ ), 3.67 (1H, ddd, J7.5, 5.5, $2.0 \mathrm{~Hz}, \mathrm{CHNH}_{2}$ ), 3.04 (1H, ddd, J 13.5, 5.5, $2.0 \mathrm{~Hz}, \mathrm{CH}), 2.83(1 \mathrm{H}$, ddd, J 13.5, 7.5, $1.5 \mathrm{~Hz}, \mathrm{CH} H), 1.49-1.44\left(2 \mathrm{H}, \mathrm{m}, \mathrm{NH}_{2}\right), 1.20(3 \mathrm{H}, \mathrm{td}$, J 7.0, $\left.1.5 \mathrm{~Hz}, \mathrm{CH}_{3}\right)$.

${ }^{13} \mathrm{C}$ NMR (101 MHz, $\mathrm{CDCl}_{3}$ ) $\delta 175.0(\mathrm{C}=\mathrm{O}), 137.3(\mathrm{Ar}), 129.3(\mathrm{Ar}), 128.5(\mathrm{Ar}), 126.7(\mathrm{Ar}), 60.9$ $\left(\mathrm{OCH}_{2}\right), 55.8\left(\mathrm{CHNH}_{2}\right), 41.1\left(\mathrm{CH}_{2}\right), 14.1\left(\mathrm{CH}_{3}\right)$.

These data are consistent with those previously reported. ${ }^{[12]}$

Amide rac-3ai was synthesised following Procedure $\mathbf{C}$, from DL-phenylalanine ethyl ester rac-1ai, to yield a colourless solid (233 mg, 77\%). NMR spectra as reported for 3ai.

HPLC analysis was then performed of 3ai and rac-3ai. 
HPLC analysis, see general experimental for conditions used.

Analytical HPLC trace of rac-3ai:

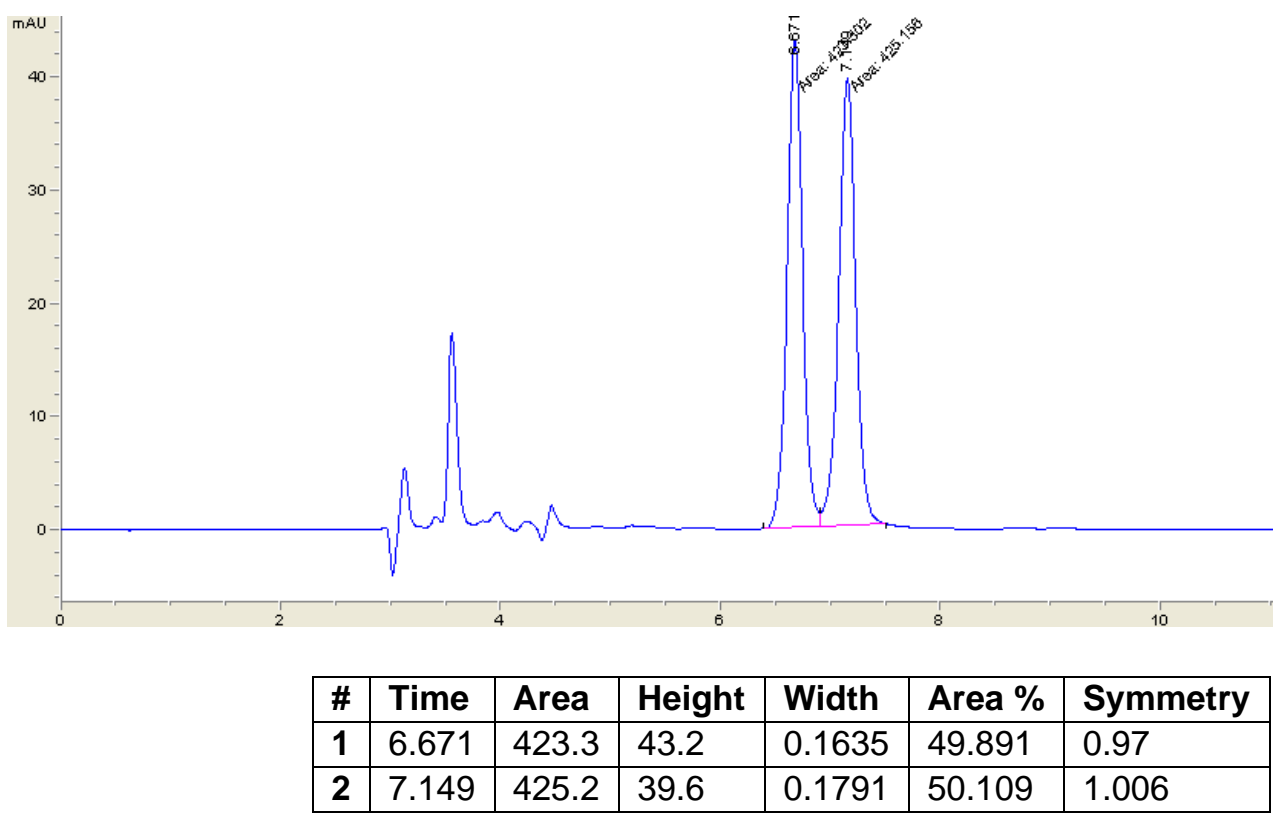

Analytical HPLC trace of 3ai:

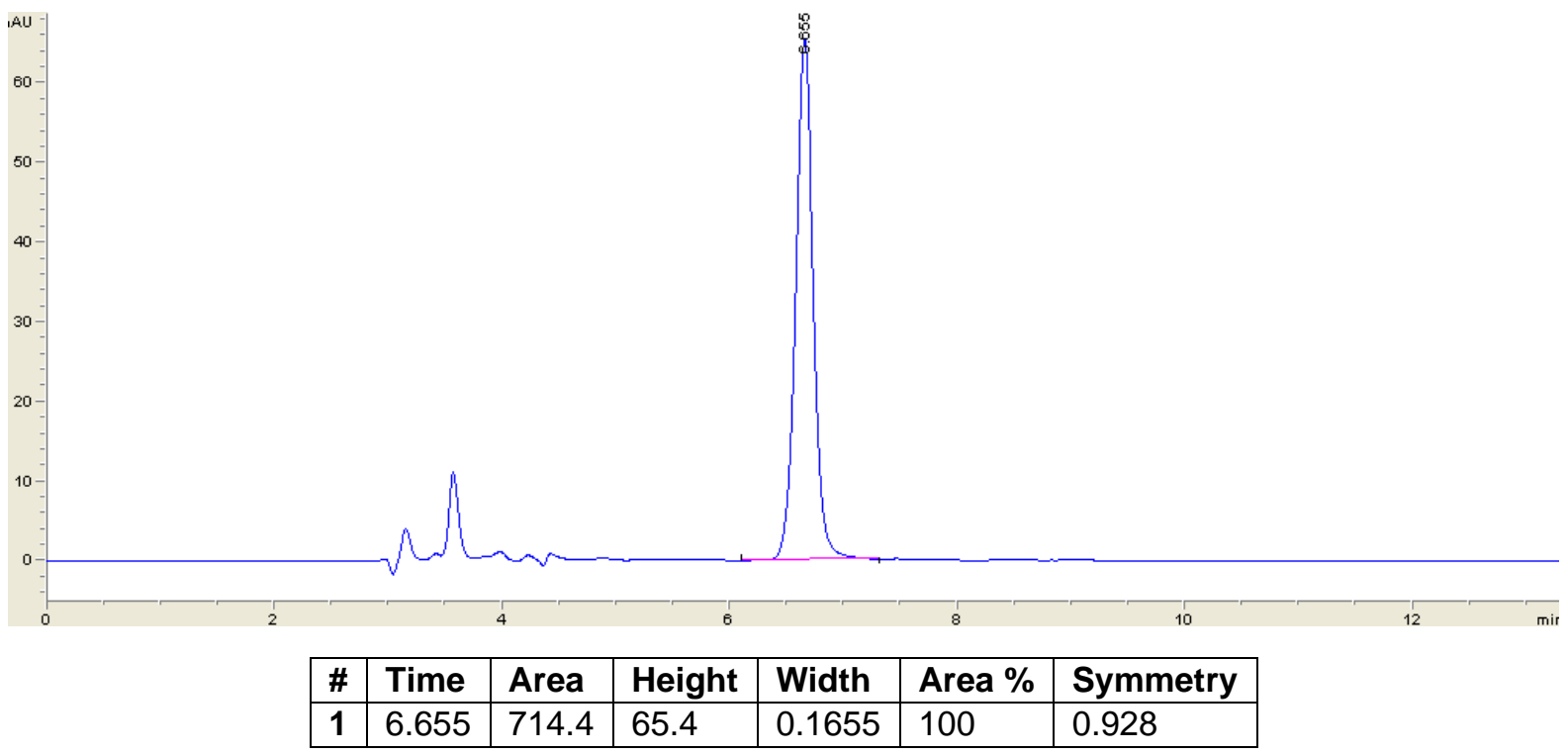

No peak at 7.149 min in the analysis of 3ai, demonstrating no erosion of ee in the dichloroacetylation of 1ai. 


\section{Unsuccessful Substrates}

Problematic substrates either presented bulky substituents adjacent to the amine or contained reactive functional groups such as alcohols or basic moieties (previously identified as problematic during reaction optimisation). In addition, substrates that were poorly soluble in ethyl acetate could not be used in this transformation.<smiles>CC(C)c1cccc(C(C)C)c1N</smiles><smiles>CC(=O)c1ccccc1N</smiles><smiles>Nc1ccccc1CCO</smiles><smiles>CC(C)(C)N</smiles><smiles>CSCCC(N)C(=O)O</smiles><smiles>Nc1ncc[nH]1</smiles><smiles>Nc1cc[nH]n1</smiles><smiles>Nc1ccccn1</smiles><smiles>Nc1ccccc1O</smiles><smiles>NCC(=O)O</smiles> 


\section{Comparison of dichloroacetylation with DiCMA versus existing literature conditions, by atom economy and complete E-factor}

Six existing literature approaches to the dichloroacetylation of amines were identified, and it was found that aniline was the common substrate to all approaches. Therefore, the reaction conditions applied to the dichloroacetylation of aniline were used for the purposes of this calculation. Where two-step approaches were considered, the amount of reagent required for the first step was scaled so as to form the exact amount of intermediate compound required for the second step. The synthesis of the reagents employed in these syntheses, for example dicyclohexylurea, dichloroacetyl chloride, and zwitterionic catalyst 5 , has not been included in the analysis. DichloroMeldrum's acid 2 could be considered either a reagent or intermediate, and so it's application to the synthesis of 2,2-dichloro- $N$-phenylacetamide $\mathbf{3 d}$ has been considered from both perspectives. Yields are calculated based on aniline as the limiting reagent.

For approaches proceeding through the intermediate 3-oxo- $N$-phenylbutanamide, formation of this through the transacetoacetylation reaction of tert-butyl acetoacetate with aniline was chosen as a representative procedure. ${ }^{[13]}$ This reaction is performed at high concentration (4 M) with no additional reagents required, and results in a good yield of 3-oxo- $N$-phenylbutanamide $(83 \%)$, with tert-butanol the only by-product formed. This therefore has a low impact on the atom economy and E-factor of the two-step approach to dichloroacetylation examined.

\section{Atom economy is calculated as:}

$$
\text { Atom Economy }=\frac{\text { Molecular Weight of Desired Product }}{\text { Molecular Weight of all Products }} \times 100 \%
$$

Catalysts are assumed to be recoverable and therefore do not contribute to the atom economy of a reaction.

\section{Complete E-Factor is calculated as:}

$$
\text { Complete E Factor }=\frac{\text { Mass of Waste Produced }}{\text { Mass of Product formed }}
$$

Water is excluded from this calculation, as per the recommended practice for the calculation of complete E-factors. ${ }^{[14]}$ Incomplete data is available for the quantity of solvents, silica, used during purification, and so purification techniques are omitted from this analysis. Chromatographic techniques required are instead noted next to each reaction below.

The data used to calculate the metrics as given in Table 2 is detailed below. 


\section{Entry 1:}

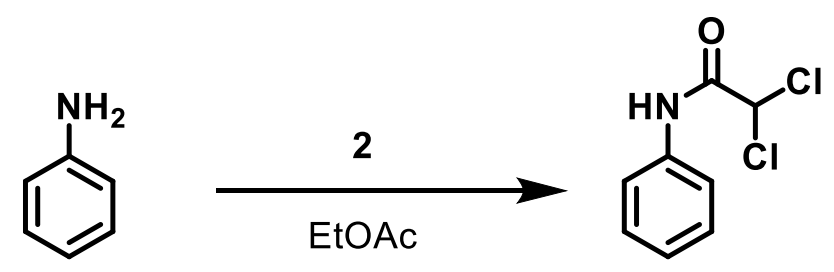

\begin{tabular}{|l|l|l|l|l|l|}
\hline Name & Aniline & $\mathbf{2}$ & Ethyl Acetate & & 3d \\
\hline Role & Starting material & Reagent & Solvent & & Product \\
\hline Mol. Wt. & 93.1 & 213.0 & 88.1 & & 204.1 \\
\hline Amount / mmol & 1.0 & 1.2 & & & 0.99 \\
\hline Amount / mg & 93.1 & 256 & 2255 & & 202 \\
\hline
\end{tabular}

Yield: 99\%

Atom Economy: $67 \%$

Complete E-factor: 12 (No chromatographic separations)

\section{Entry S1:}

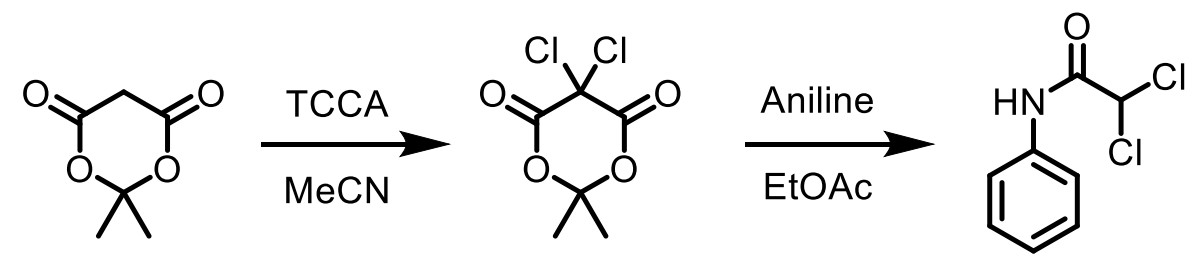

\begin{tabular}{|l|l|l|l|l|l|l|}
\hline Name & $\begin{array}{l}\text { Meldrum's } \\
\text { Acid }\end{array}$ & TCCA & Acetonitrile & Aniline & $\begin{array}{l}\text { Ethyl } \\
\text { Acetate }\end{array}$ & 3d \\
\hline Role & $\begin{array}{l}\text { Starting } \\
\text { material }\end{array}$ & Reagent & Solvent & $\begin{array}{l}\text { Starting } \\
\text { material }\end{array}$ & Solvent & Product \\
\hline Mol. Wt. & 144.1 & 232.4 & 41.1 & 93.1 & 88.1 & 204.1 \\
\hline Amount / mmol & 1.32 & 1.44 & & 1.0 & & 0.99 \\
\hline Amount / mg & 190.0 & 369 & 2987 & 93.1 & 2255 & 202 \\
\hline
\end{tabular}

Yield: 99\%

Atom Economy: $43 \%$

Complete E-factor: 28 (No chromatographic separations) 


\section{Entry 2:}

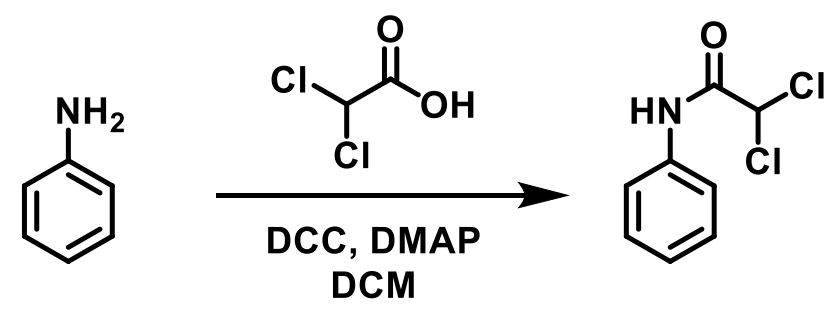

Column Chromatography used

\begin{tabular}{|l|l|l|l|l|l|l|}
\hline Name & Aniline & $\begin{array}{l}\text { Dichloroacetic } \\
\text { acid }\end{array}$ & DMAP & DCC & DCM & 3d \\
\hline Role & $\begin{array}{l}\text { Starting } \\
\text { material }\end{array}$ & Reagent & Catalyst & Reagent & Solvent & Product \\
\hline Mol. Wt. & 93.1 & 128.9 & 122.2 & 206.3 & 84.9 & 204.1 \\
\hline Amount / mmol & 1.0 & 1.2 & 0.16 & 1.2 & - & 0.95 \\
\hline Amount / mg & 93.1 & 155 & 19.6 & 247.6 & 26500 & 194 \\
\hline
\end{tabular}

Yield: 95\%

Atom Economy: $48 \%$

Complete E-factor: 138 (Excluding column chromatography)

\section{Entry 3:}

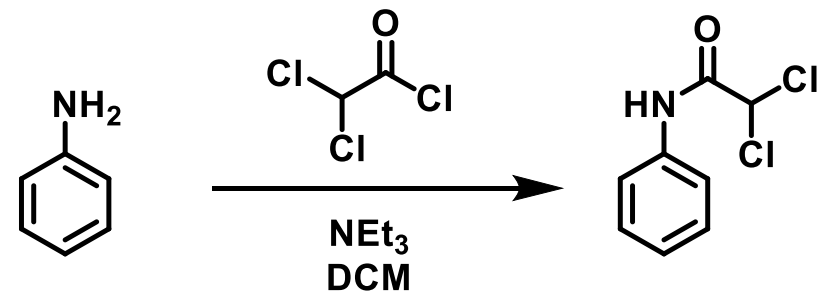

Column Chromatography used

\begin{tabular}{|l|l|l|l|l|l|l|}
\hline Name & Aniline & $\begin{array}{l}\text { Dichloroacetyl } \\
\text { chloride }\end{array}$ & Triethylamine & & DCM & 3d \\
\hline Role & $\begin{array}{l}\text { Starting } \\
\text { material }\end{array}$ & Reagent & Reagent & & Solvent & Product \\
\hline Mol. Wt. & 93.1 & 128.9 & 101.2 & & 84.9 & 204.1 \\
\hline Amount / mmol & 10 & 11 & 12 & & - & 8.6 \\
\hline Amount / mg & 930 & 1621 & 1214 & & 26500 & 1750 \\
\hline
\end{tabular}

Yield: $86 \%$

Atom Economy: 60\%

Complete E-factor: 16 (Excluding column chromatography) 


\section{Entry 4:}

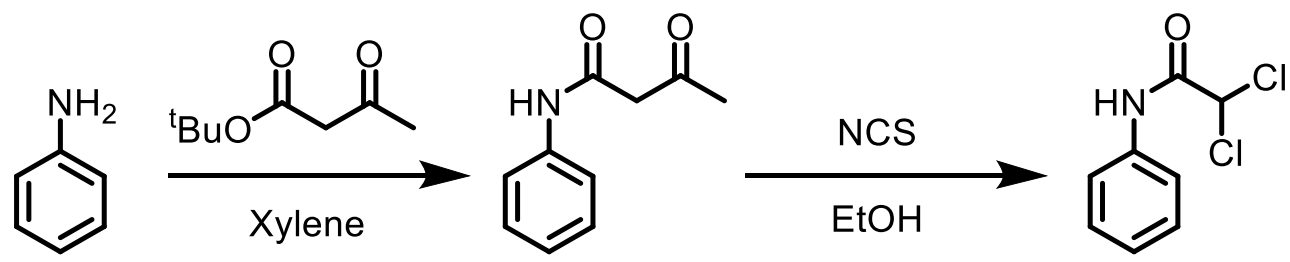

\begin{tabular}{|l|l|l|l|l|l|l|}
\hline Name & Aniline & 'BuAcAc & Xylene & $\begin{array}{l}\text { N- } \\
\text { Chlorosuccinimide }\end{array}$ & Ethanol & 3d \\
\hline Role & $\begin{array}{l}\text { Starting } \\
\text { Material }\end{array}$ & Reagent & Solvent & Reagent & Solvent & Product \\
\hline Mol. Wt. & 93.1 & 158.2 & 106.2 & 133.5 & 46.1 & 204.1 \\
\hline Amount $/ \mathbf{~ m m o l ~}$ & 1.2 & 1.22 & & 2.2 & & 0.83 \\
\hline Amount $/ \mathbf{m g}$ & 112 & 192 & 241 & 294 & 15780 & 169 \\
\hline
\end{tabular}

Yield: $69 \%$

Atom Economy: 53\%

Complete E-factor: 97 (No chromatographic separations)

\section{Entry 5:}<smiles>Nc1ccccc1</smiles>

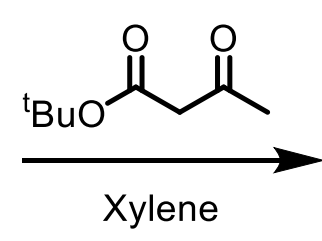<smiles>CC(=O)CC(=O)Nc1ccccc1</smiles><smiles>O=C(Nc1ccccc1)C(Cl)Cl</smiles>

Column Chromatography used

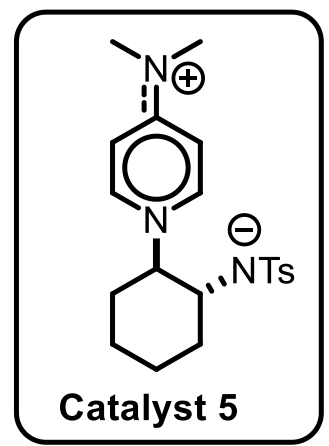

\begin{tabular}{|l|l|l|l|l|l|l|l|}
\hline Name & Aniline & tBuAcAc & Xylene & $\begin{array}{l}\text { N- } \\
\text { Chlorosuccinimide }\end{array}$ & $\begin{array}{c}\text { Catalyst } \\
\mathbf{5}\end{array}$ & Methanol & 3d \\
\hline Role & $\begin{array}{l}\text { Starting } \\
\text { Material }\end{array}$ & Reagent & Solvent & Reagent & Catalyst & Solvent & Product \\
\hline Mol. Wt. & 93.1 & 158.2 & 106.2 & 133.5 & 373.5 & 32.0 & 204.1 \\
\hline $\begin{array}{l}\text { Amount } \\
\text { / mmol }\end{array}$ & 0.36 & 0.36 & & 0.63 & 0.015 & & 0.27 \\
\hline Amount / mg & 33.6 & 57.7 & 72.2 & 84.1 & 5.6 & 4746 & 55 \\
\hline
\end{tabular}

Yield: 75\%

Atom Economy: 53\%

Complete E-factor: 90 (Excluding column chromatography) 


\section{Entry 6:}<smiles>CC(=O)CC(=O)Nc1ccccc1NC(=O)CC(C)COC(=O)CC(C)=O</smiles>

Preparative TLC used

\begin{tabular}{|l|l|l|l|l|l|l|l|}
\hline Name & Aniline & 'BuAcAc & Xylene & $\begin{array}{l}\text { Zinc(II) } \\
\text { Chloride }\end{array}$ & Phl(OAc) & Dioxane & 3d \\
\hline Role & $\begin{array}{l}\text { Starting } \\
\text { Material }\end{array}$ & Reagent & Solvent & Reagent & Reagent & Solvent & Product \\
\hline Mol. Wt. & 93.1 & 158.2 & 106.2 & 136.3 & 322.1 & 88.1 & 204.1 \\
\hline Amount / mmol & 1.2 & 1.22 & & 1.5 & 1.3 & & 0.84 \\
\hline Amount / mg & 112 & 192 & 241 & 204 & 419 & 2068 & 171 \\
\hline
\end{tabular}

Yield: $70 \%$

Atom Economy: 29\%

Complete E-factor: 18 (Excluding preparative TLC)

\section{Entry 7:}

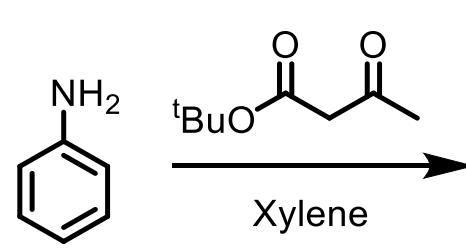<smiles>CC(=O)CC(=O)Nc1ccccc1</smiles>

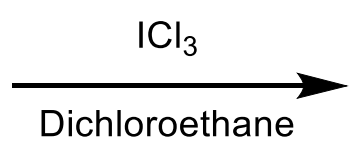<smiles>O=C(Nc1ccccc1)C(Cl)Cl</smiles>

Preparative TLC used

\begin{tabular}{|l|l|l|l|l|l|l|}
\hline Name & Aniline & tBuAcAc & Xylene & $\begin{array}{l}\text { lodine } \\
\text { Trichloride }\end{array}$ & Dichloroethane & 3d \\
\hline Role & $\begin{array}{l}\text { Starting } \\
\text { Material }\end{array}$ & Reagent & Solvent & Reagent & Solvent & Product \\
\hline Mol. Wt. & 93.1 & 158.2 & 106.2 & 233.3 & 99.0 & 204.1 \\
\hline Amount / mmol & 1.2 & 1.22 & & 1.3 & & 0.84 \\
\hline Amount / mg & 112 & 192 & 241 & 303 & 2512 & 171 \\
\hline
\end{tabular}

Yield: $72 \%$

Atom Economy: $42 \%$

Complete E-factor: 18 (Excluding preparative TLC) 


\section{NMR Spectra}

DichloroMeldrum's Acid 2

${ }^{1} \mathrm{H}$ NMR (400 MHz, $\mathrm{CDCl}_{3}$ )

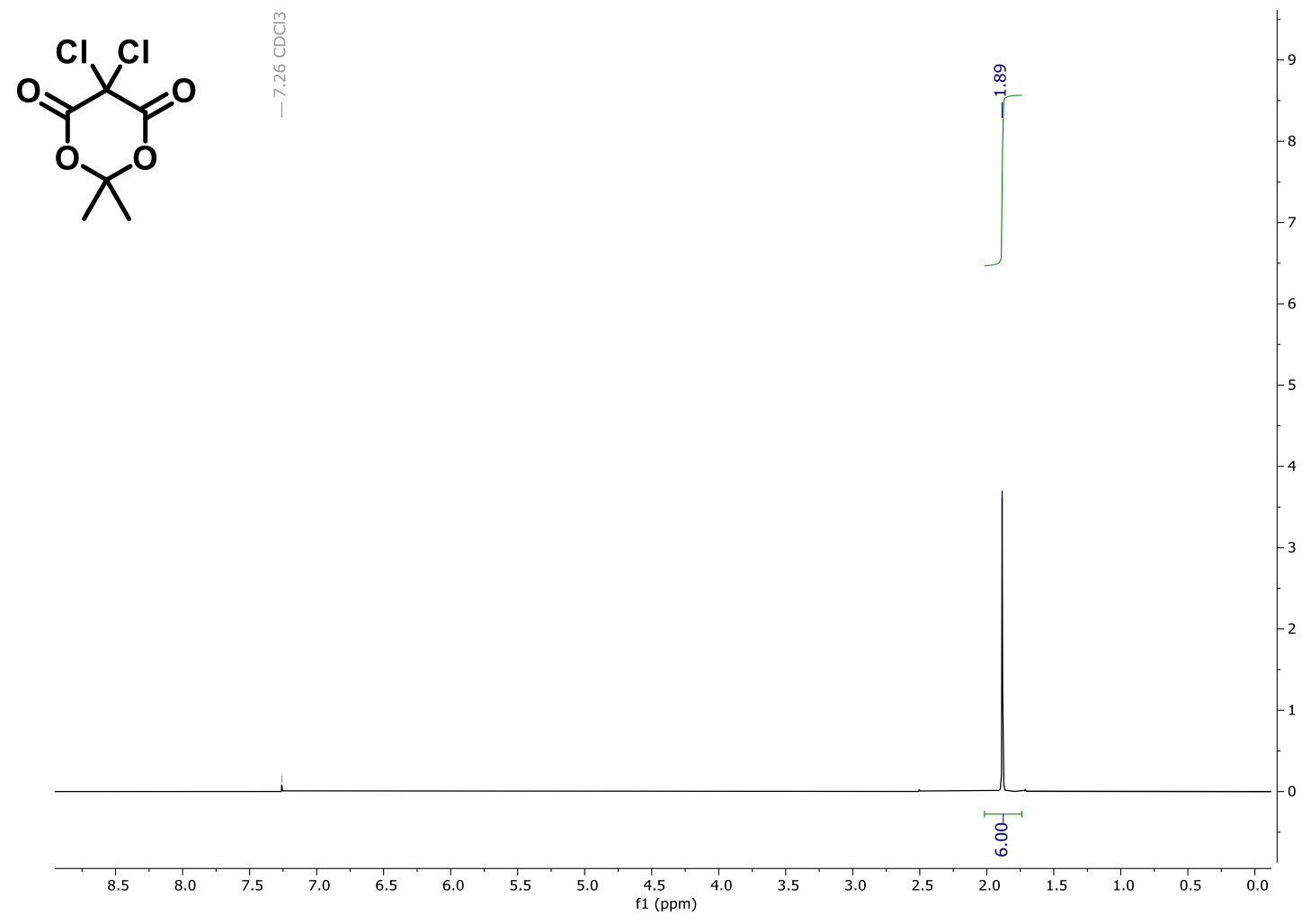


${ }^{13} \mathrm{C}$ NMR (101 MHz, $\mathrm{CDCl}_{3}$ )

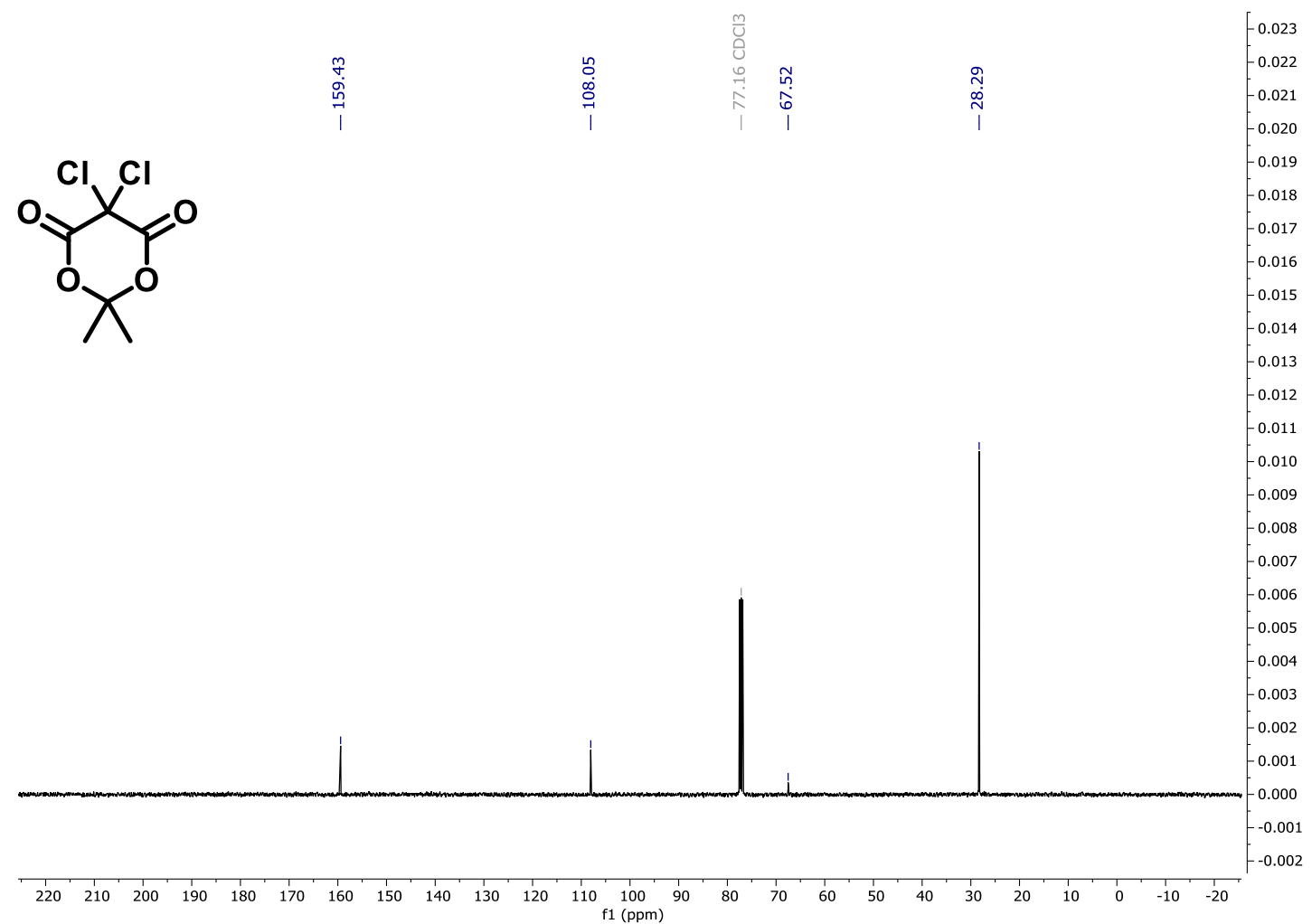


2,2-Dichloro- $N$-(4-fluorophenyl)acetamide 3a

${ }^{1} \mathrm{H}$ NMR (400 MHz, $\mathrm{CDCl}_{3}$ )

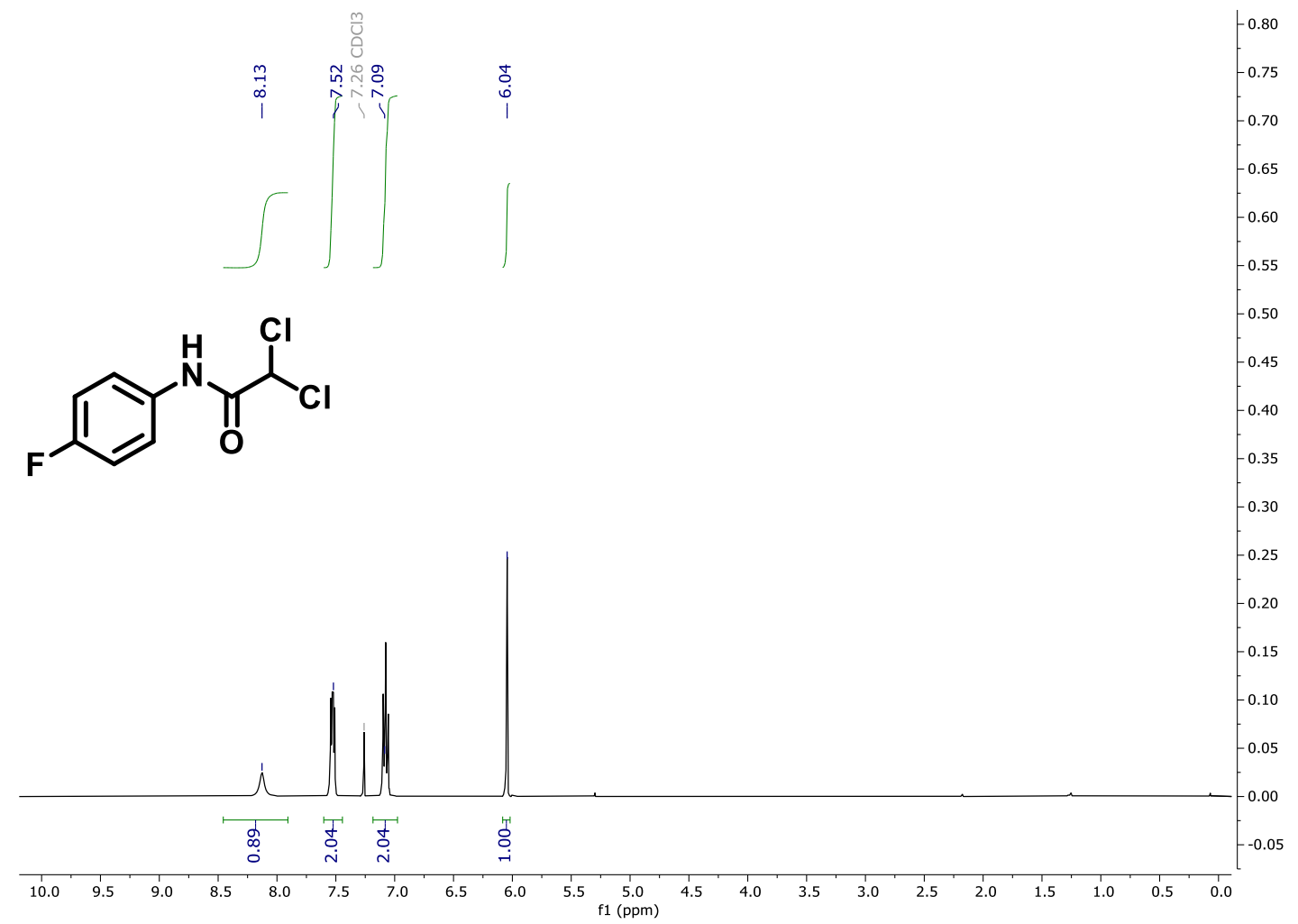


${ }^{13} \mathrm{C}$ NMR (101 MHz, $\mathrm{CDCl}_{3}$ )

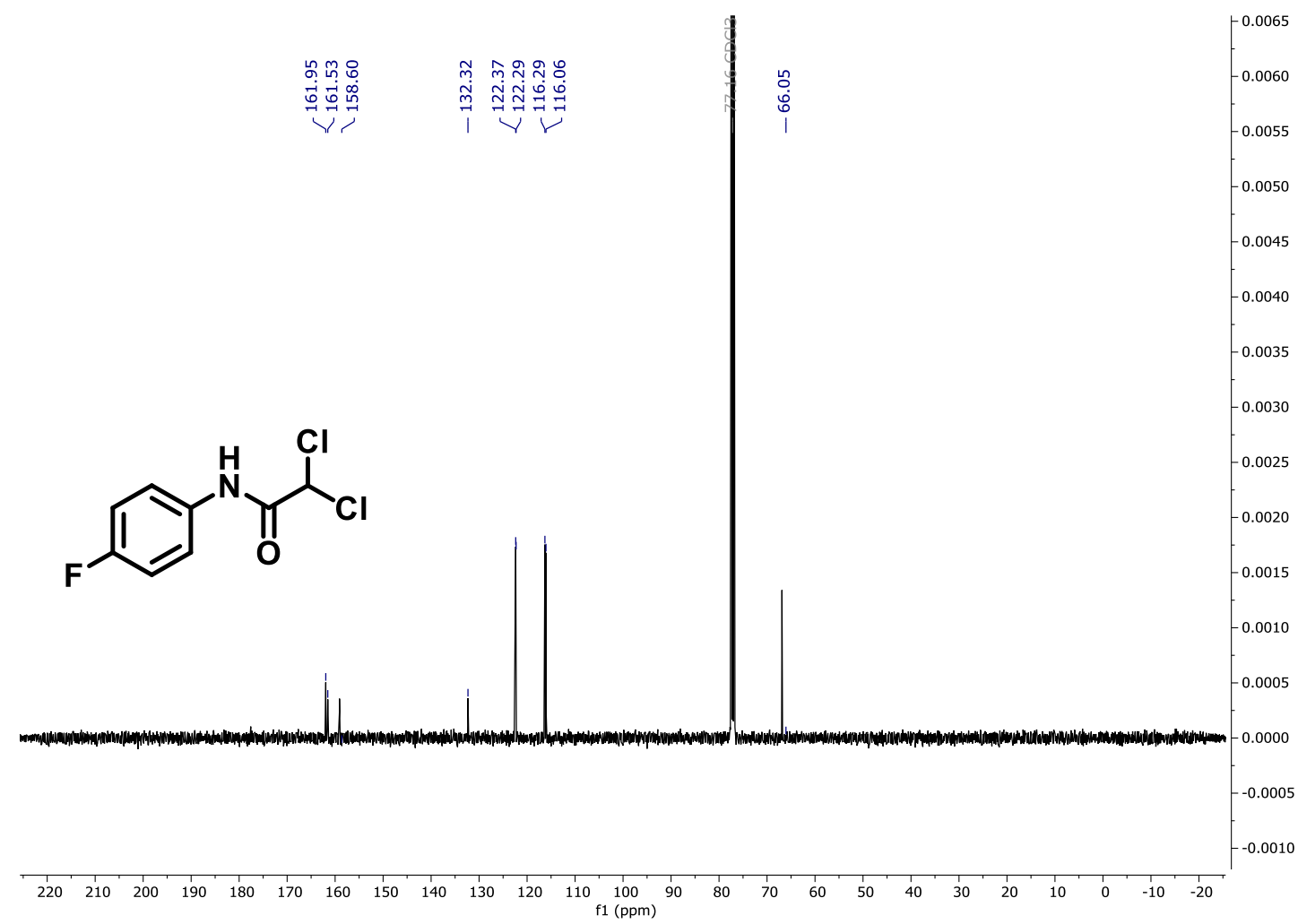


${ }^{19} \mathrm{~F} \mathrm{NMR} \mathrm{(376} \mathrm{MHz,} \mathrm{CDCl}_{3}$ )

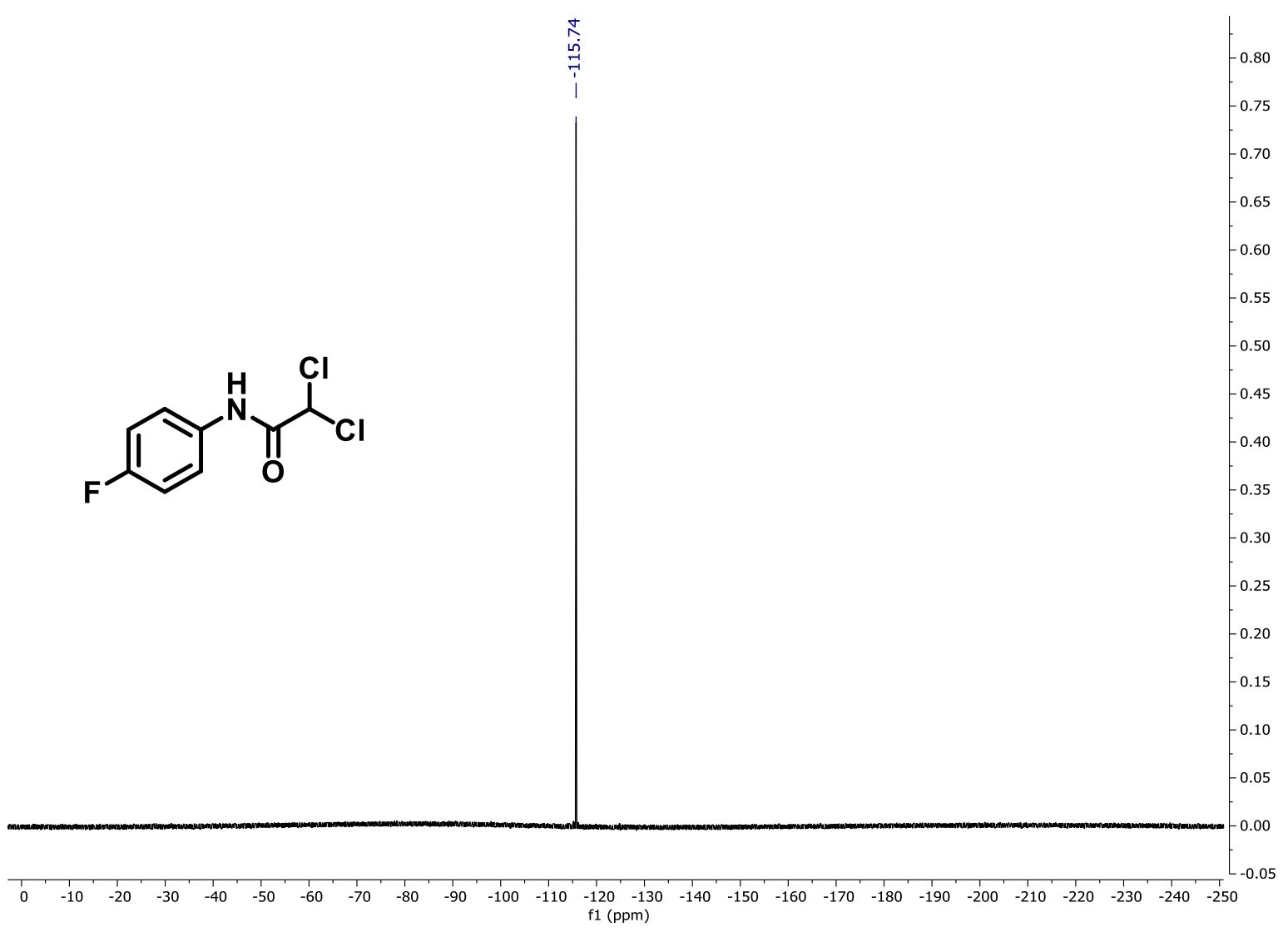


2,2-Dichloro- $N$-(4-methoxyphenyl)acetamide 3b

${ }^{1} \mathrm{H}$ NMR $\left(400 \mathrm{MHz}, \mathrm{CDCl}_{3}\right)$

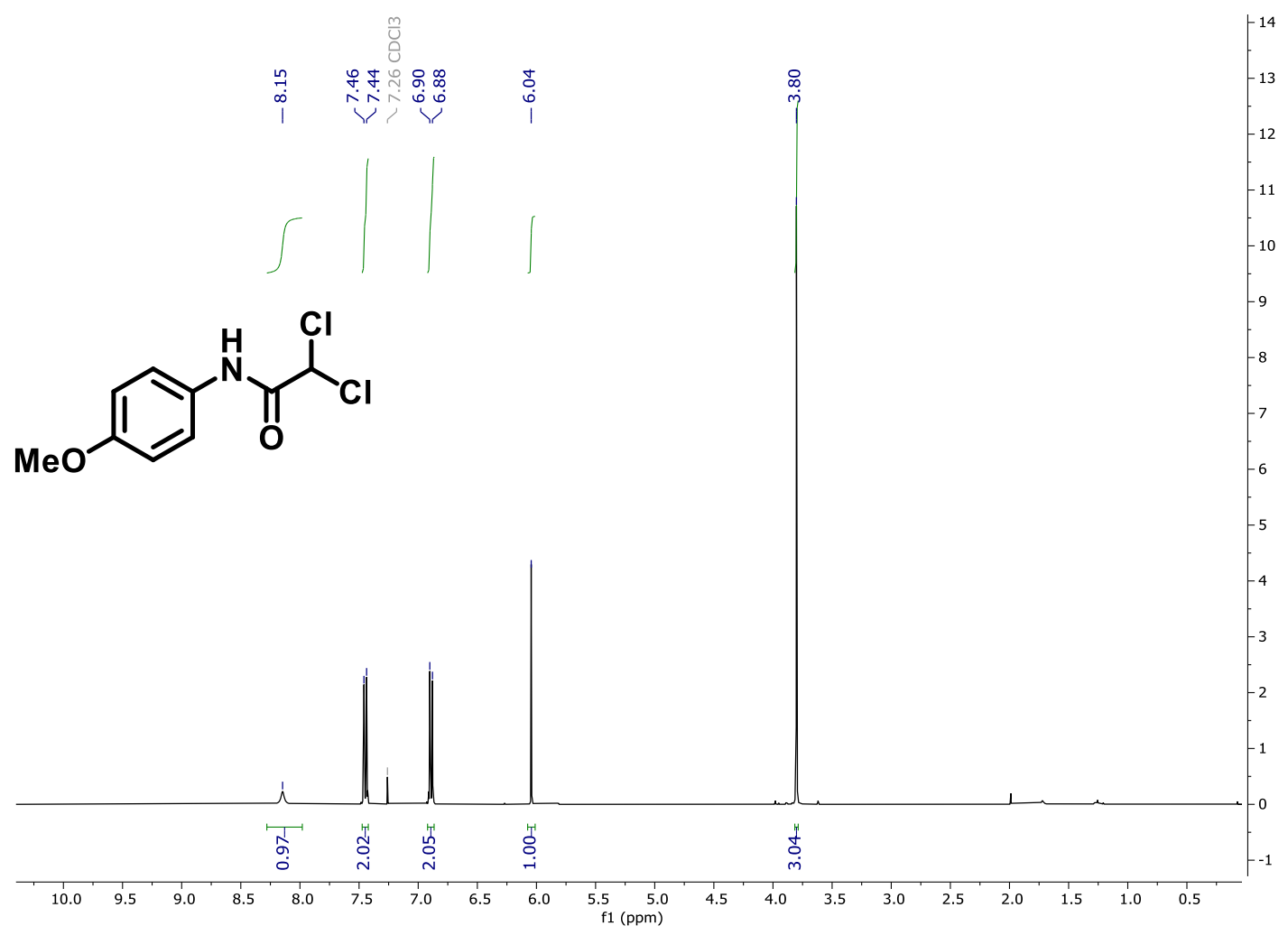


${ }^{13} \mathrm{C}$ NMR (101 MHz, $\mathrm{CDCl}_{3}$ )

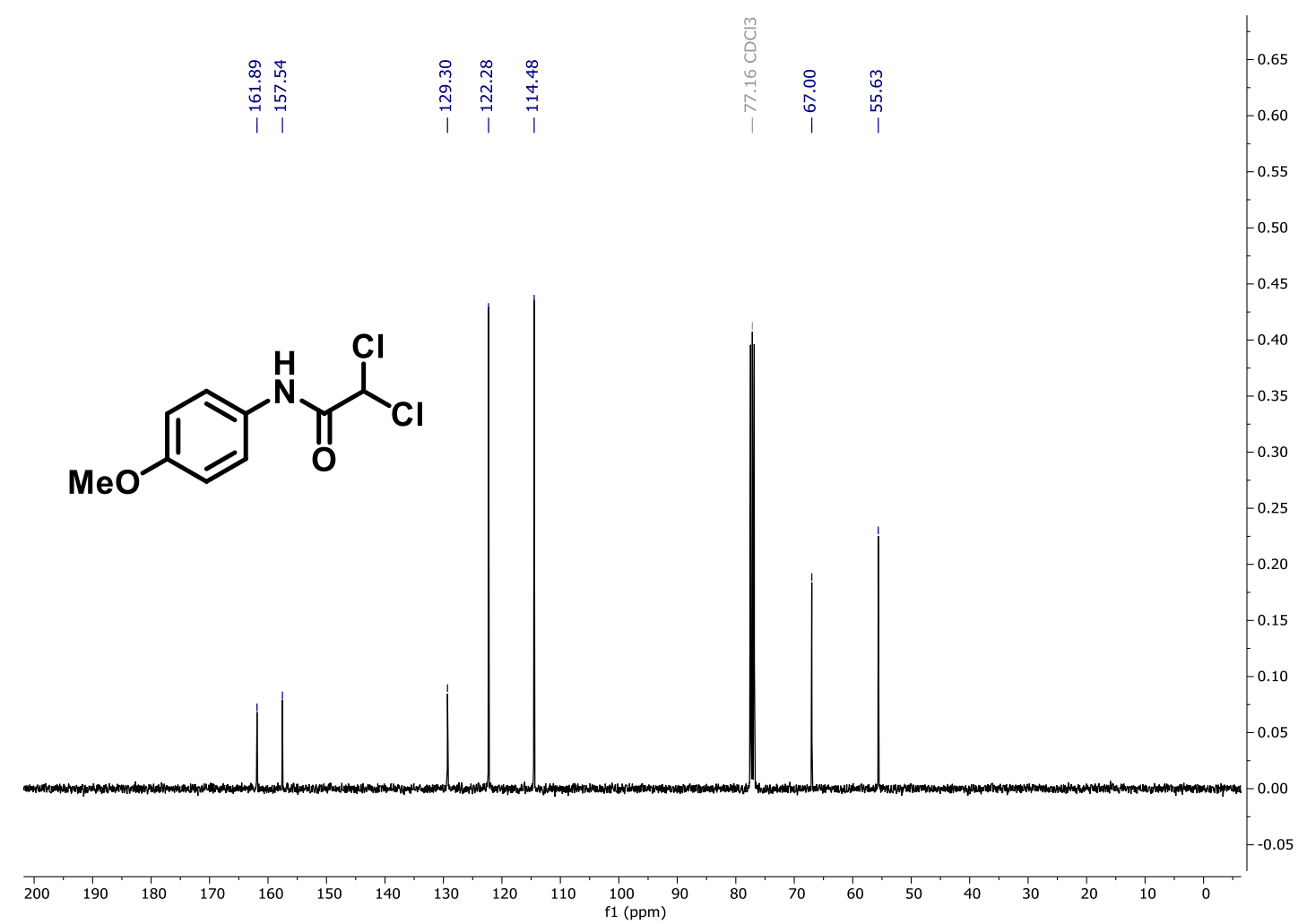


2,2-Dichloro- $N$-(4-methylphenyl)acetamide 3c

${ }^{1} \mathrm{H}$ NMR (400 MHz, $\mathrm{CDCl}_{3}$ )

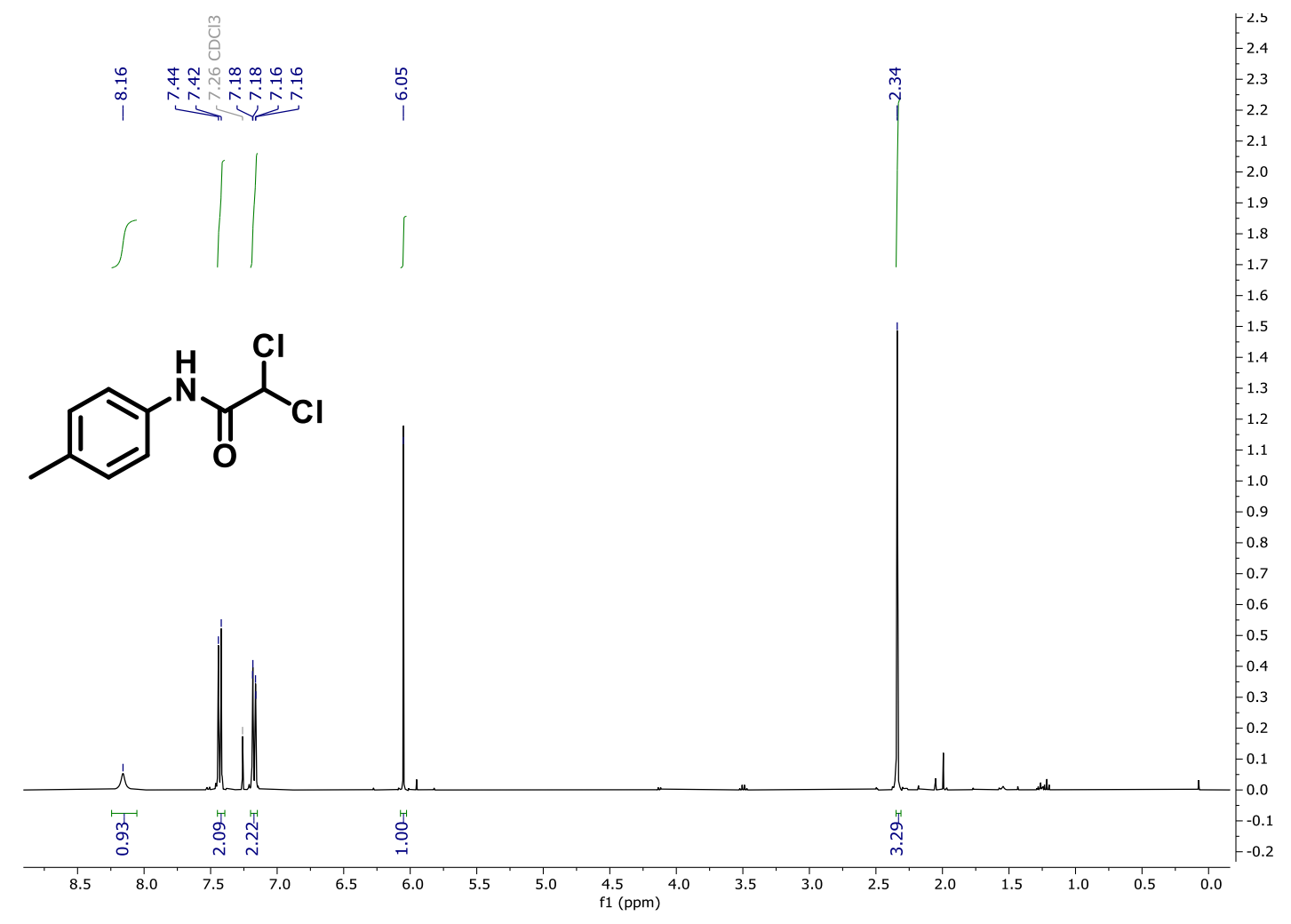


${ }^{13} \mathrm{C}$ NMR (101 MHz, $\mathrm{CDCl}_{3}$ )

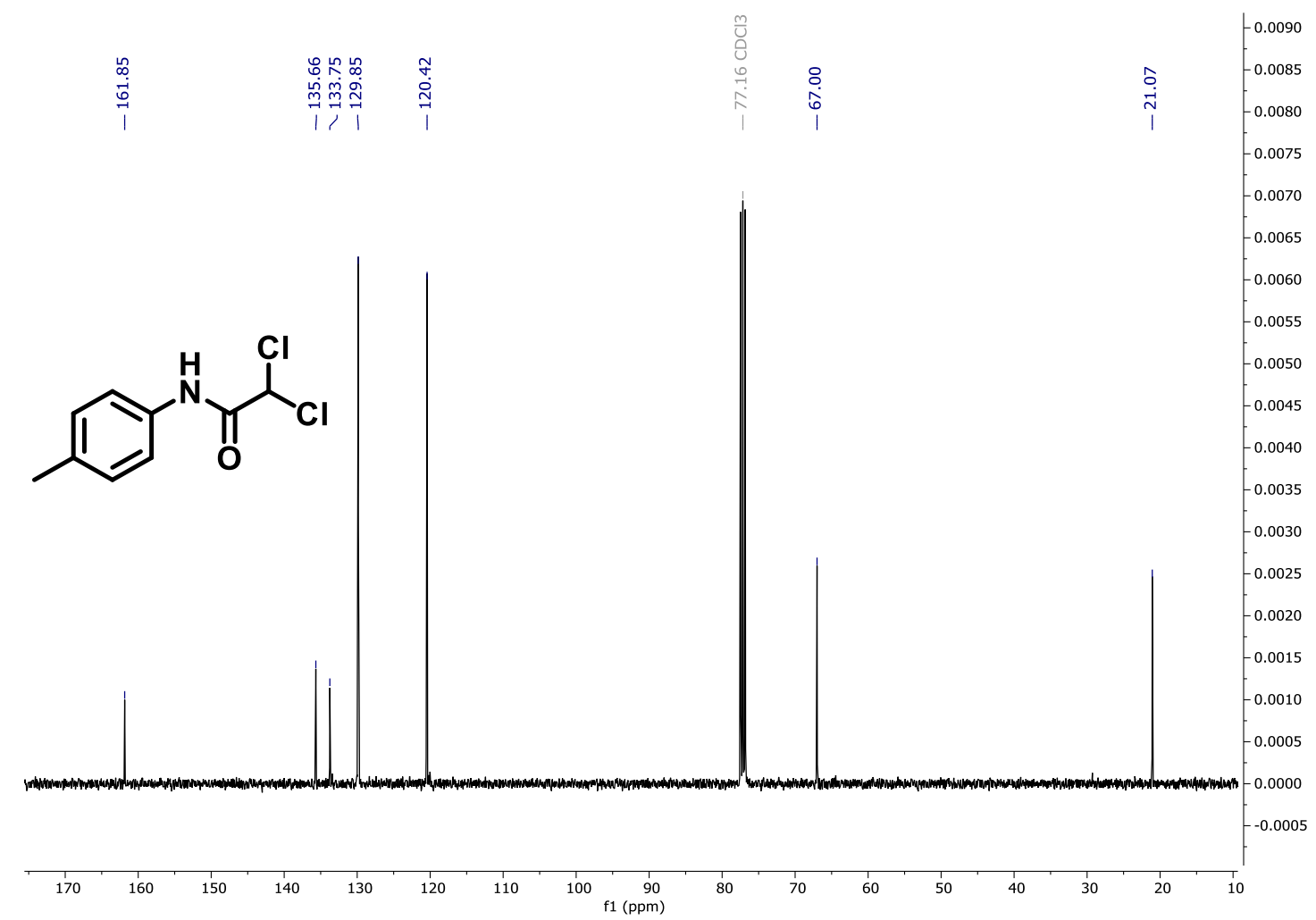


2,2-Dichloro- $N$-phenylacetamide 3d

${ }^{1} \mathrm{H}$ NMR (400 MHz, $\mathrm{CDCl}_{3}$ )

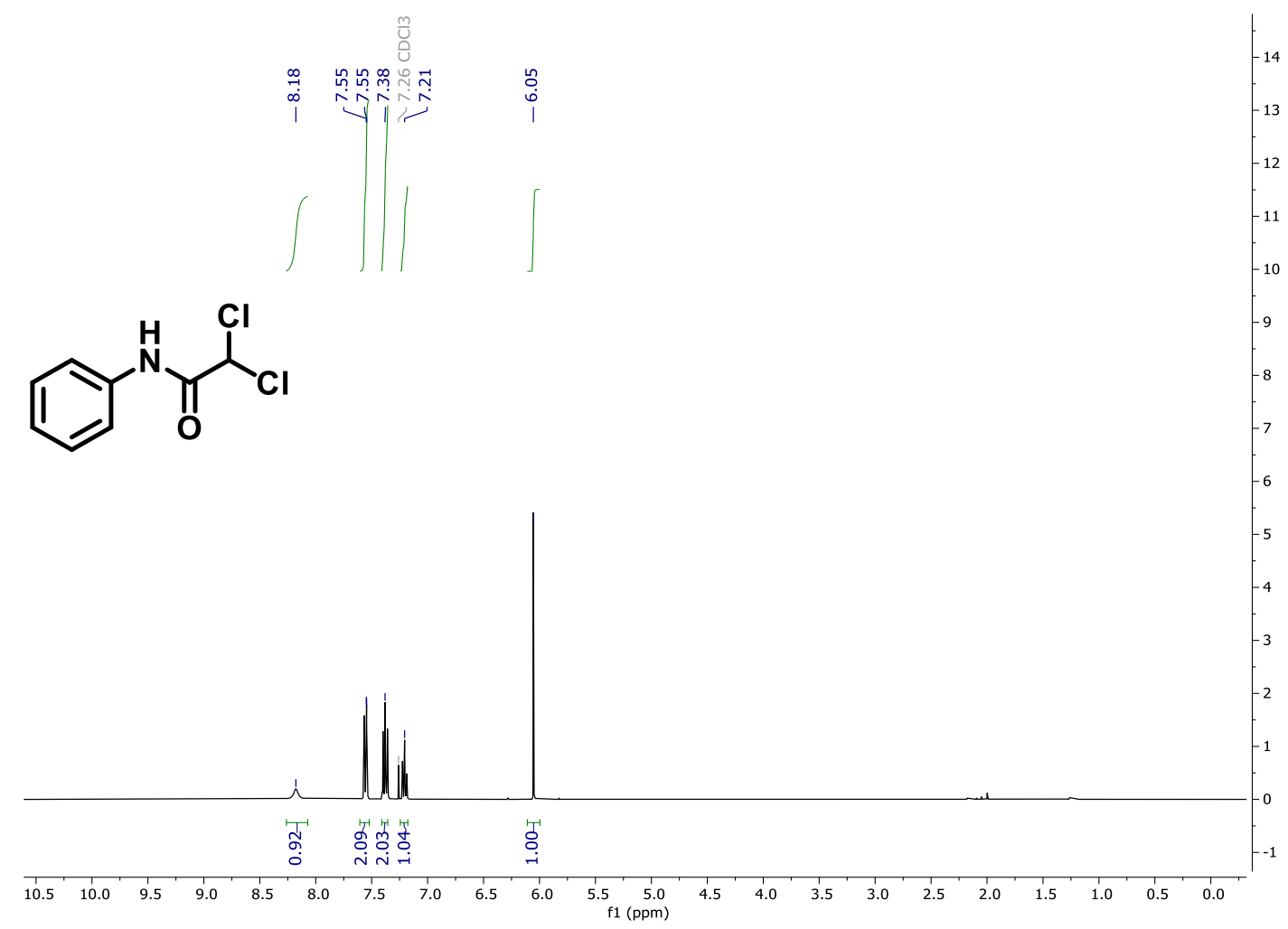


${ }^{13} \mathrm{C}$ NMR (101 MHz, $\mathrm{CDCl}_{3}$ )

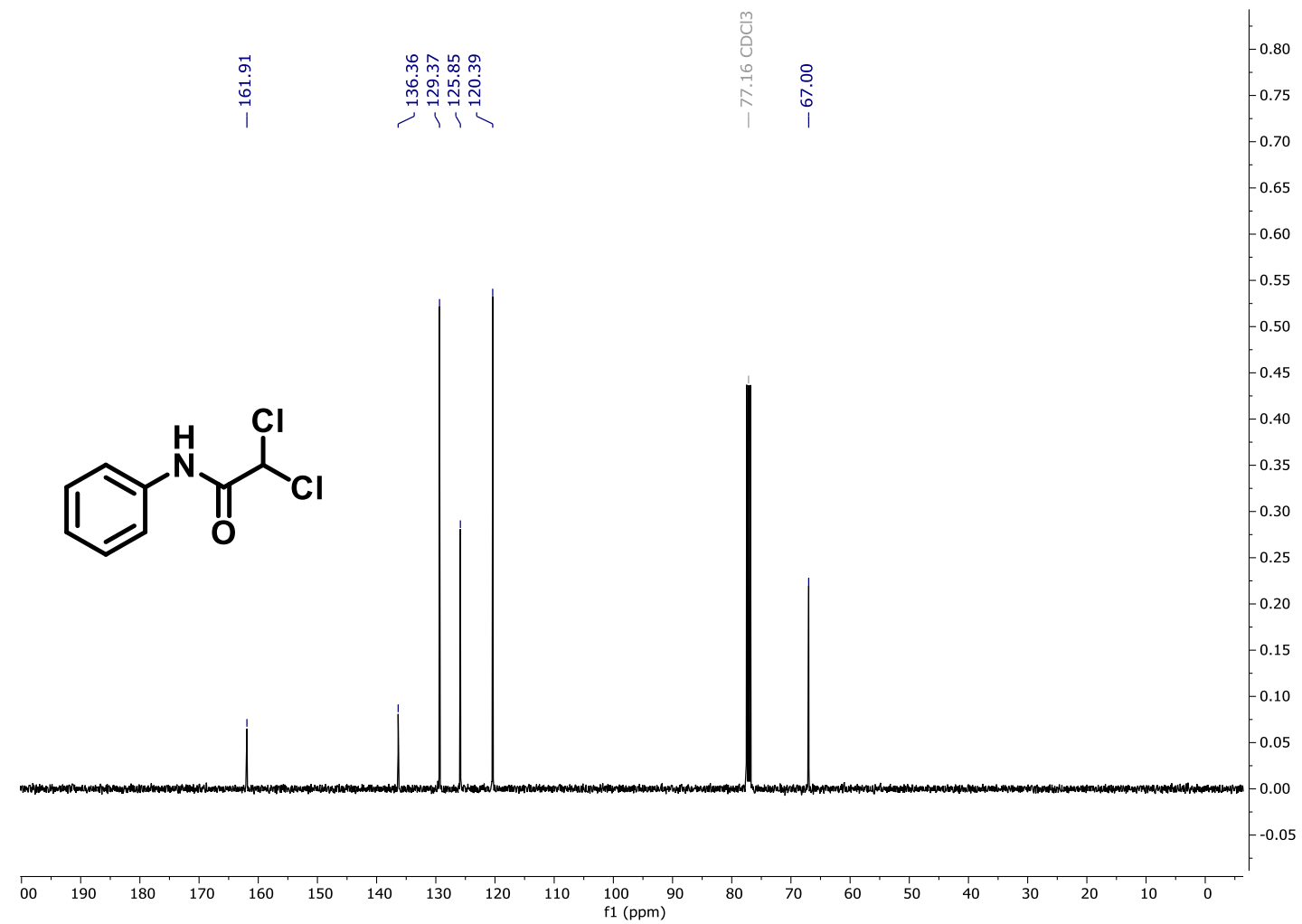


2,2-Dichloro- $N$-(4-trifluoromethylphenyl)acetamide $3 e$ ${ }^{1} \mathrm{H}$ NMR (400 MHz, $\left.\mathrm{CDCl}_{3}\right)$

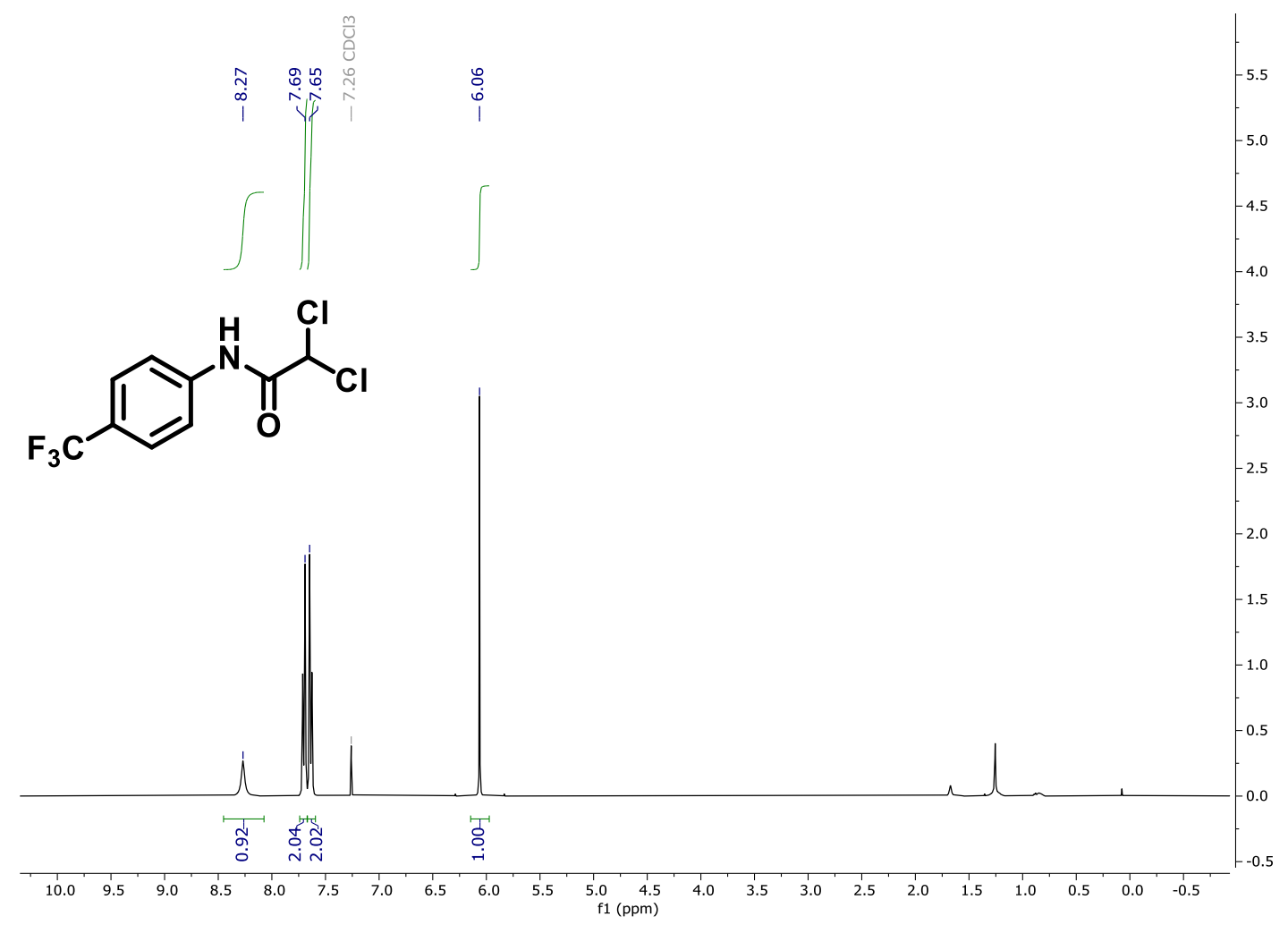


${ }^{13} \mathrm{C}$ NMR (101 MHz, $\left.\mathrm{CDCl}_{3}\right)$

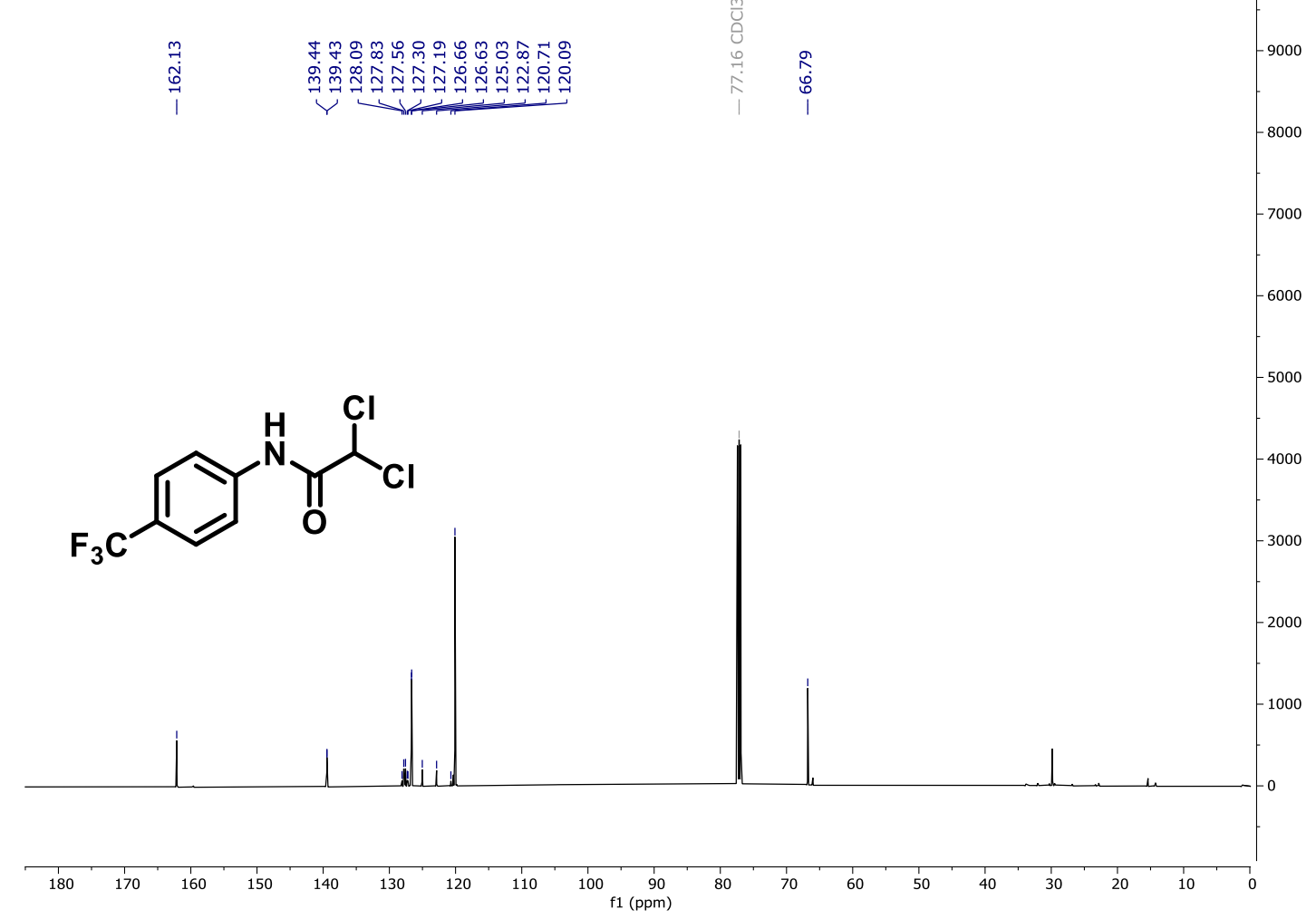


${ }^{19} \mathrm{~F}$ NMR (376 MHz, $\mathrm{CDCl}_{3}$ )

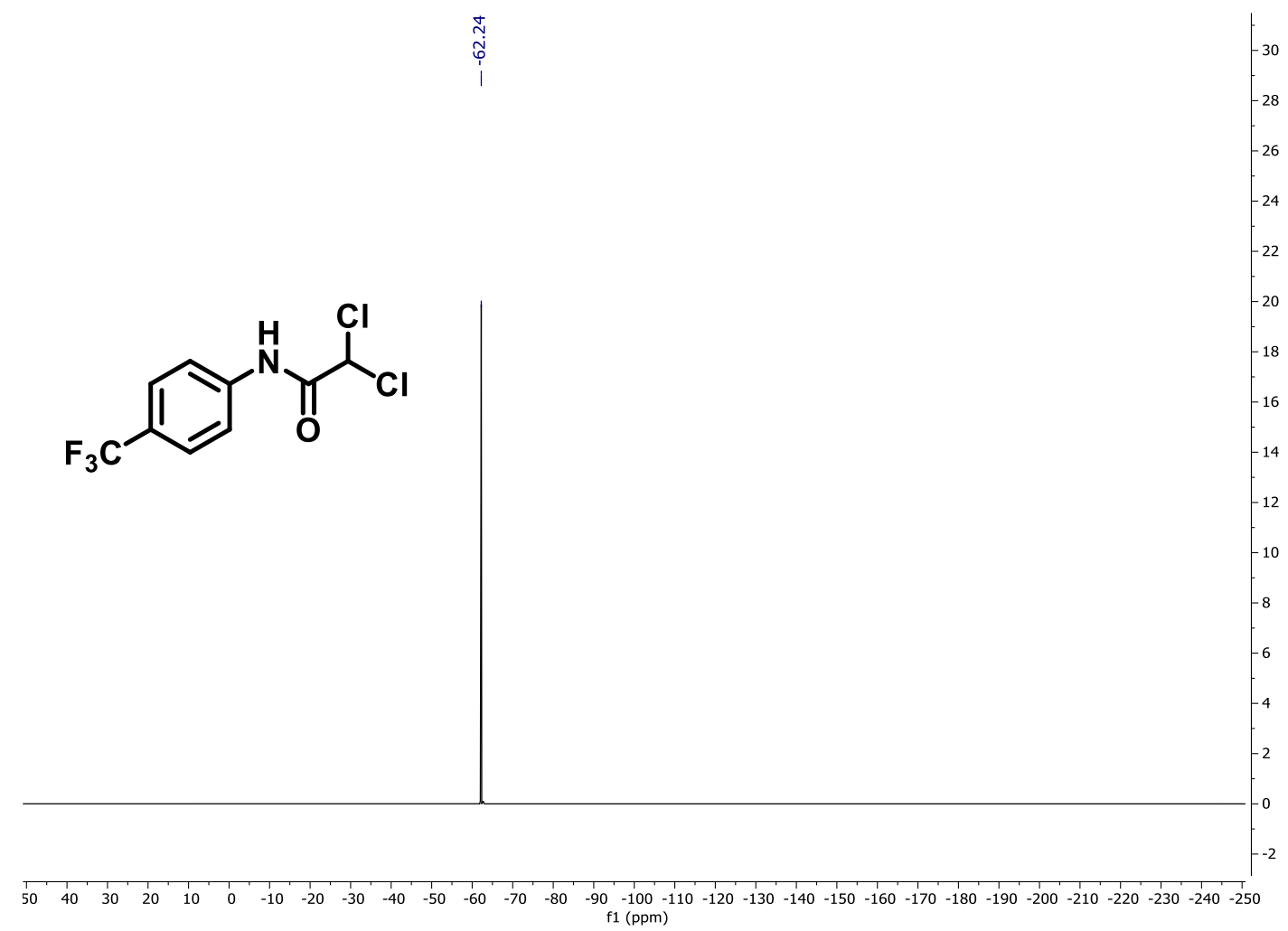


$\mathrm{N}$-(4-Benzoylphenyl)-2,2-dichloroacetamide $3 f$

${ }^{1} \mathrm{H}$ NMR (400 MHz, $\left.\mathrm{CDCl}_{3}\right)$

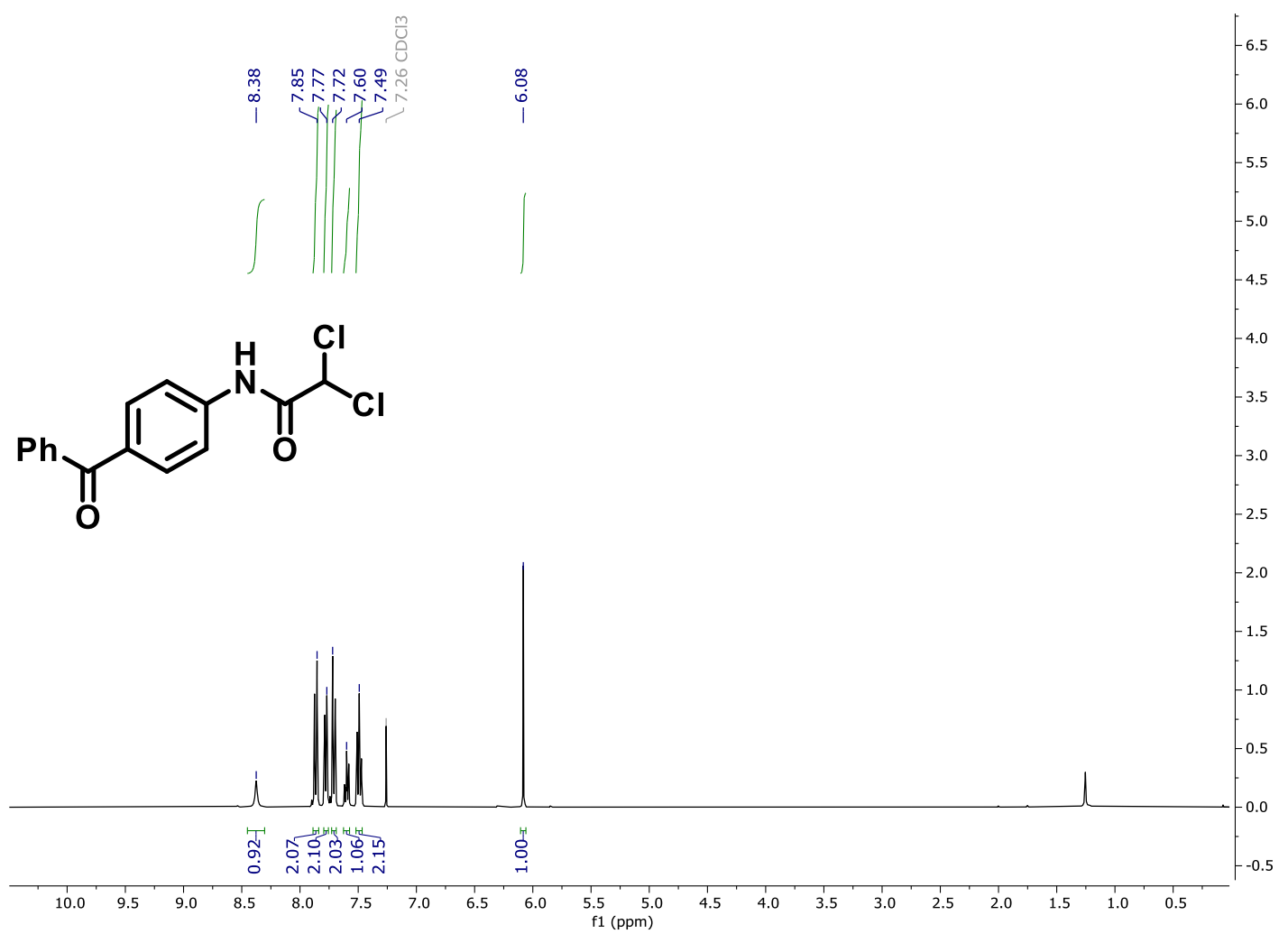


${ }^{13} \mathrm{C}$ NMR (101 MHz, $\left.\mathrm{CDCl}_{3}\right)$

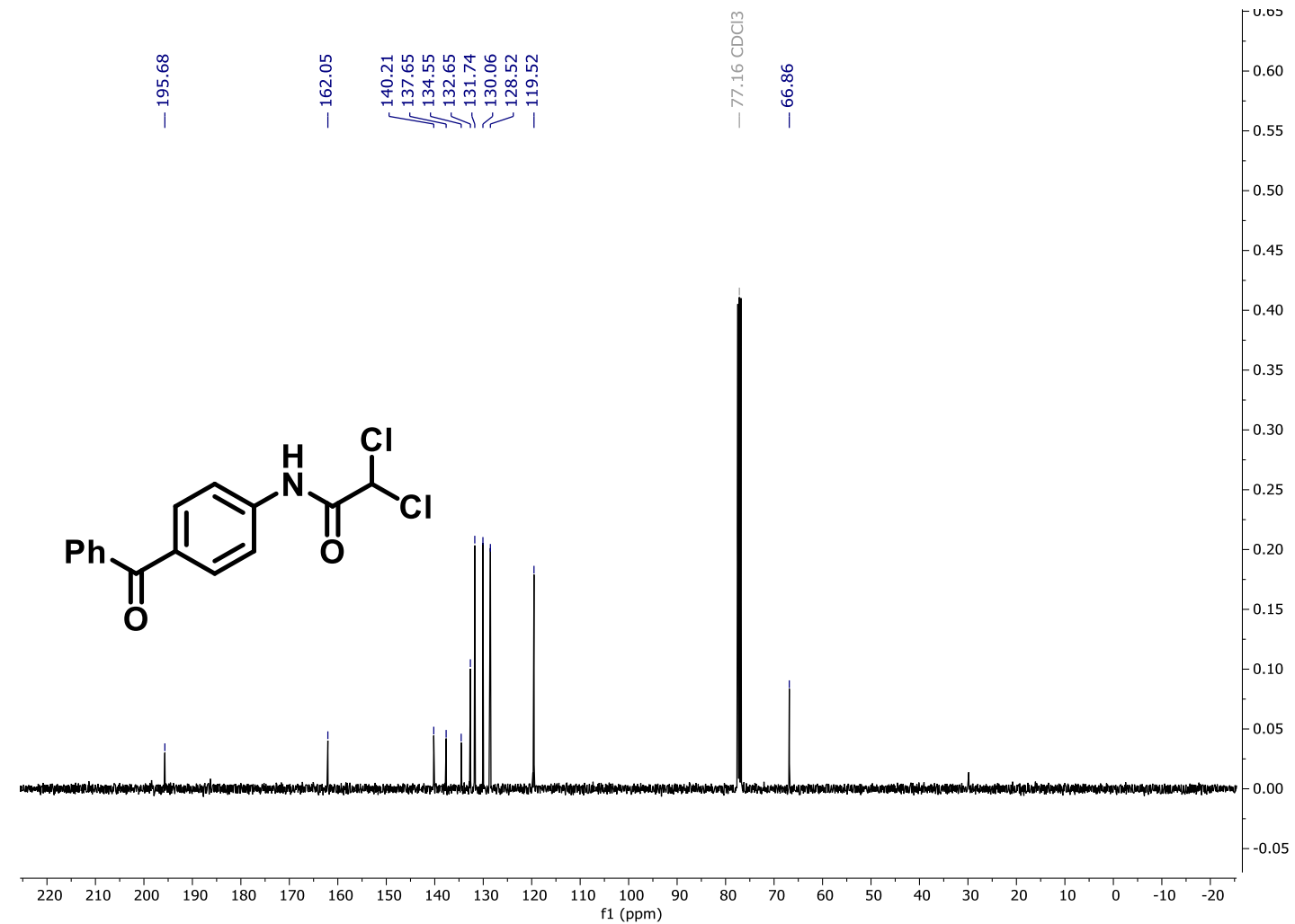


2,2-Dichloro- $N$-(2-fluorophenyl)acetamide $3 g$

${ }^{1} \mathrm{H}$ NMR (400 MHz, $\mathrm{CDCl}_{3}$ )

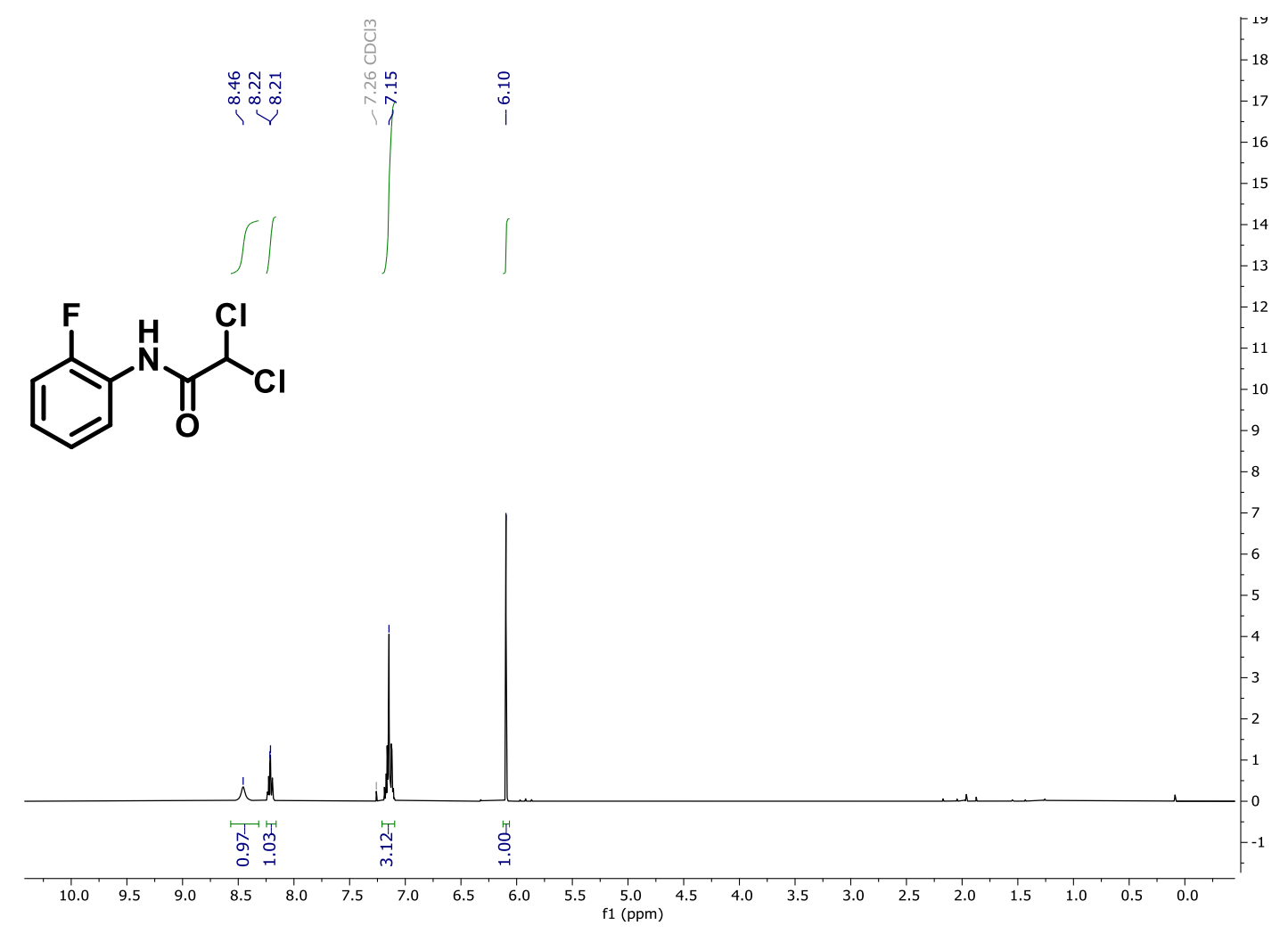


${ }^{13} \mathrm{C}$ NMR (101 MHz, $\left.\mathrm{CDCl}_{3}\right)$

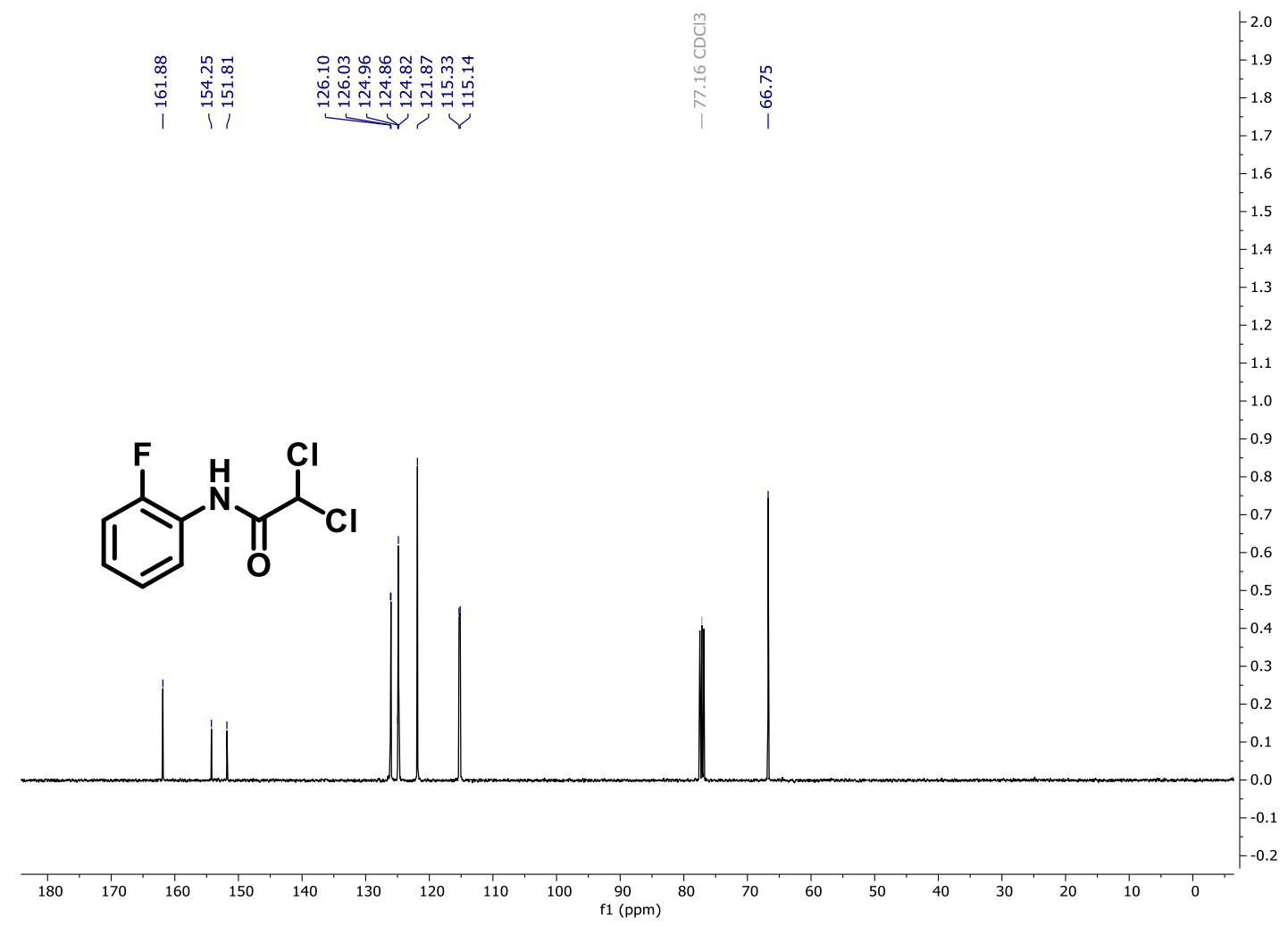


${ }^{19} \mathrm{~F}$ NMR (376 MHz, $\mathrm{CDCl}_{3}$ )

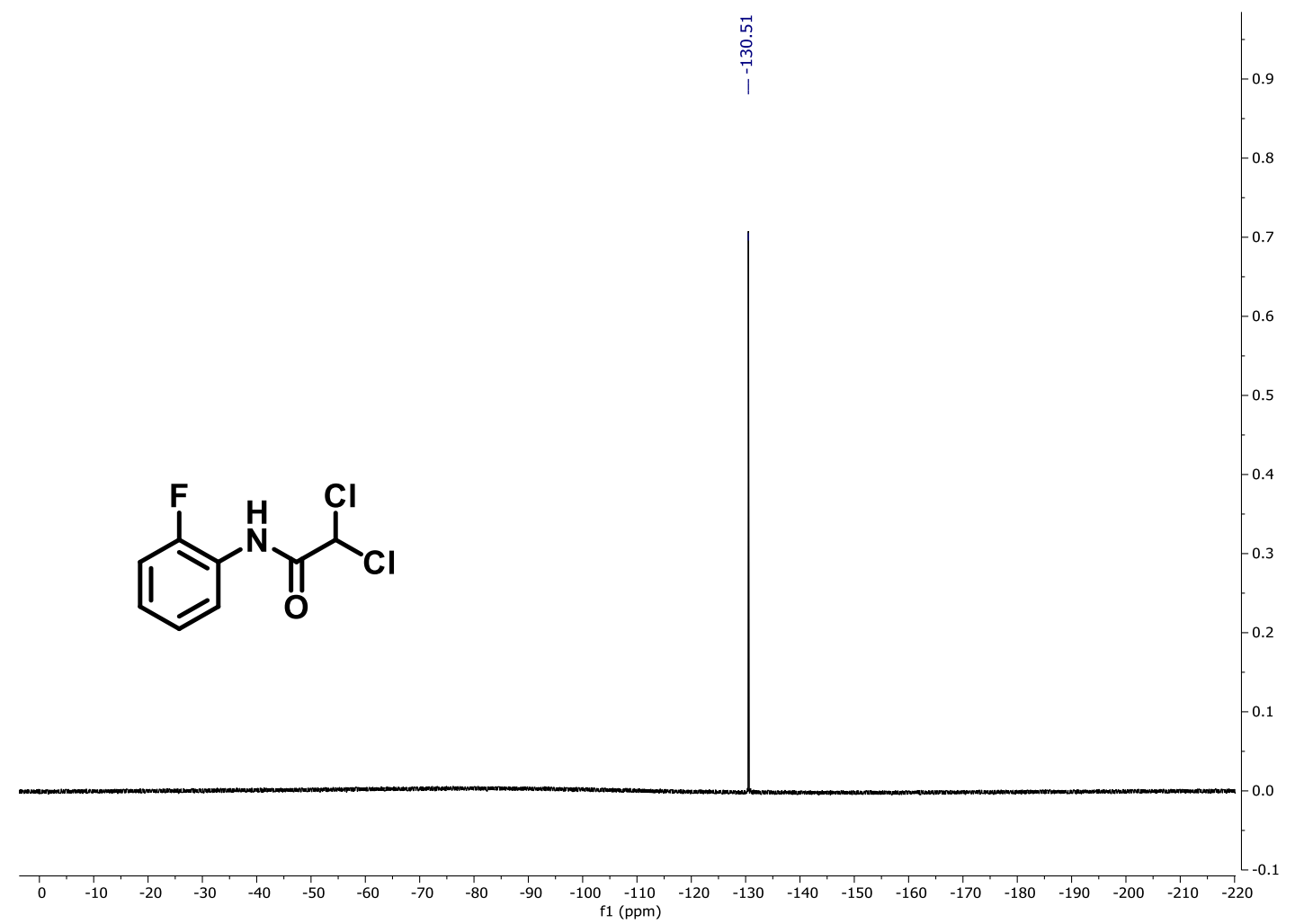


2,2-Dichloro- $\mathrm{N}$-(2-chlorophenyl)acetamide $3 \mathrm{~h}$

${ }^{1} \mathrm{H} \mathrm{NMR}\left(400 \mathrm{MHz}, \mathrm{CDCl}_{3}\right)$

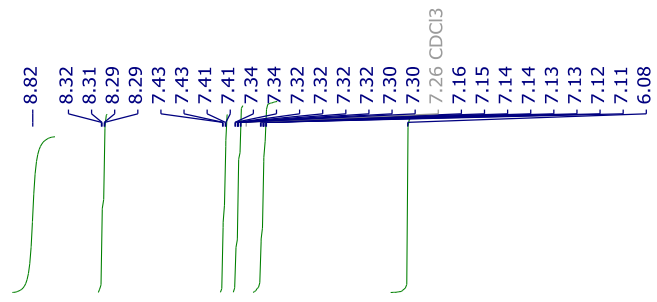

$\underbrace{C l}_{0}$

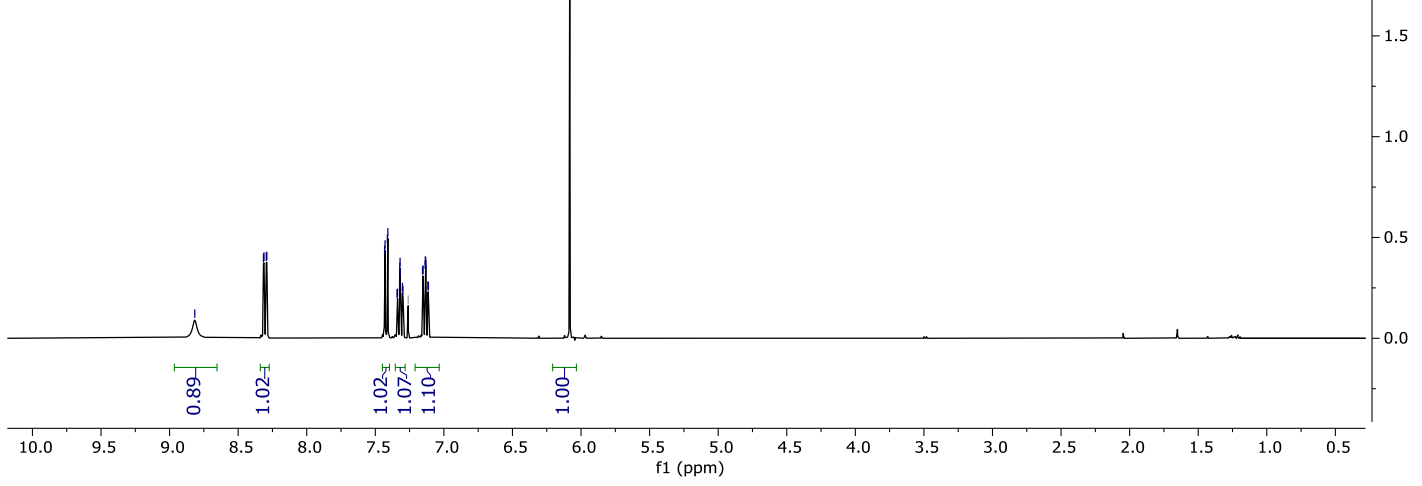


${ }^{13} \mathrm{C}$ NMR (101 MHz, $\left.\mathrm{CDCl}_{3}\right)$

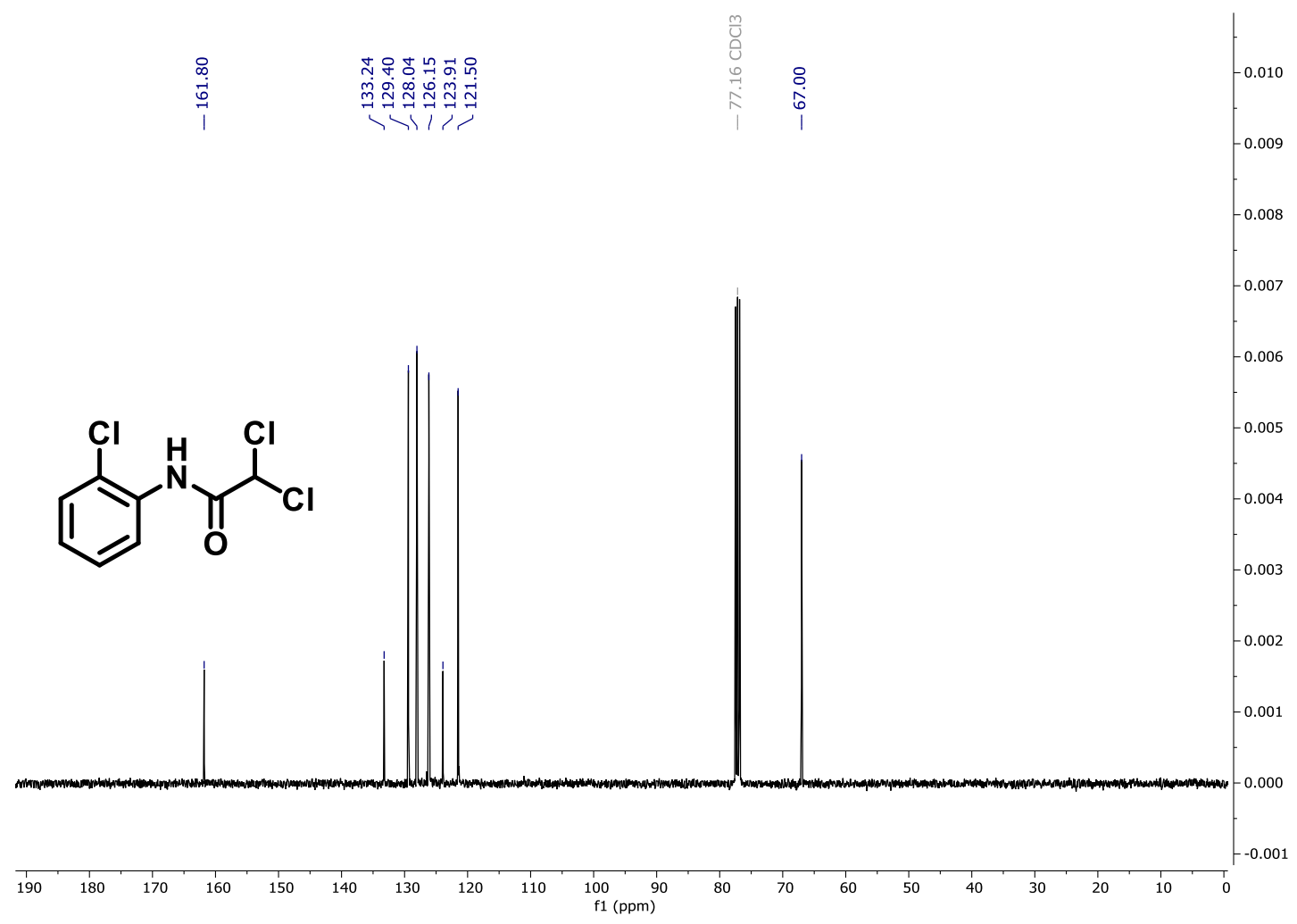

S59 
2,2-Dichloro- $N$-(2-methoxyphenyl)acetamide 3i

${ }^{1} \mathrm{H}$ NMR (400 MHz, $\mathrm{CDCl}_{3}$ )

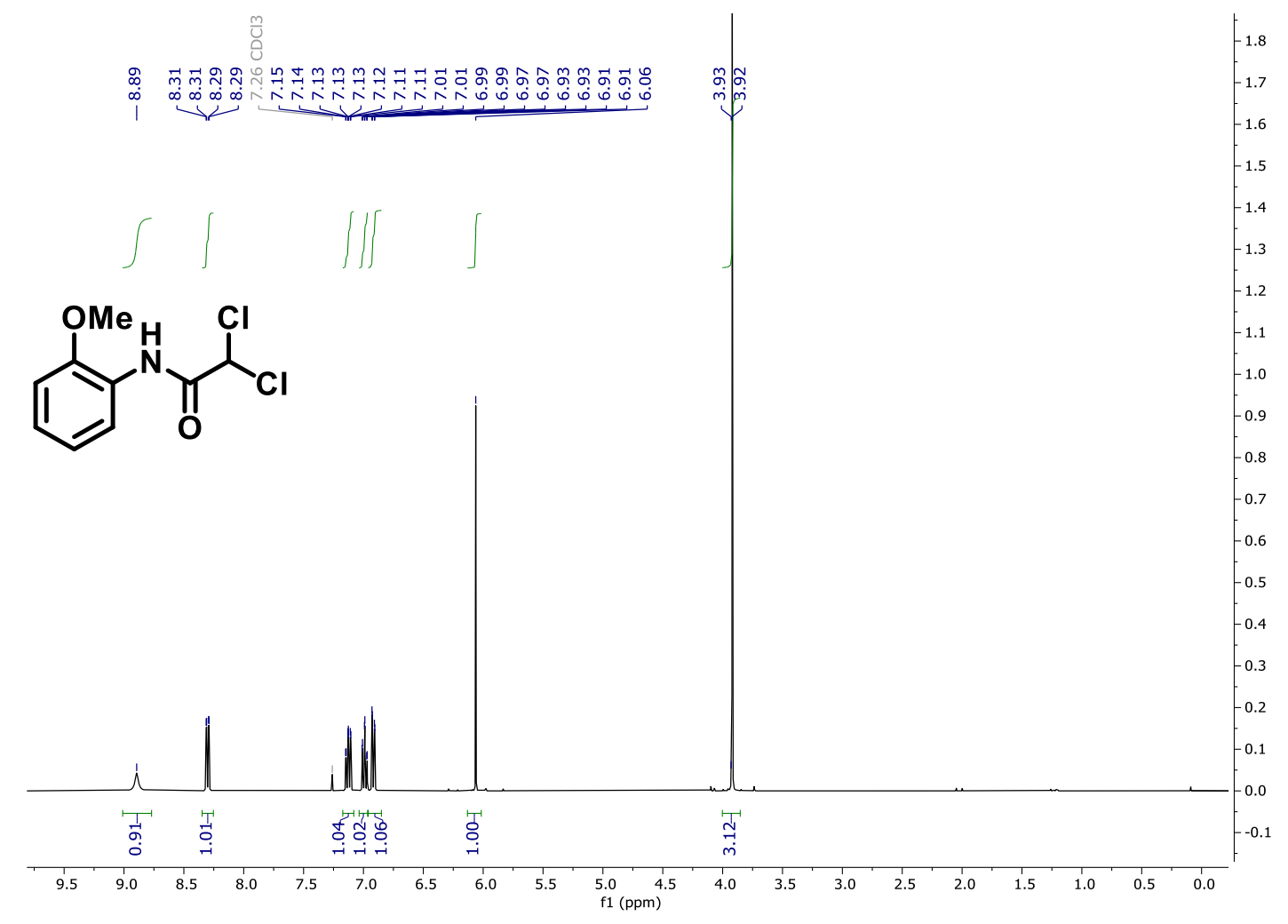


${ }^{13} \mathrm{C}$ NMR (101 MHz, $\left.\mathrm{CDCl}_{3}\right)$

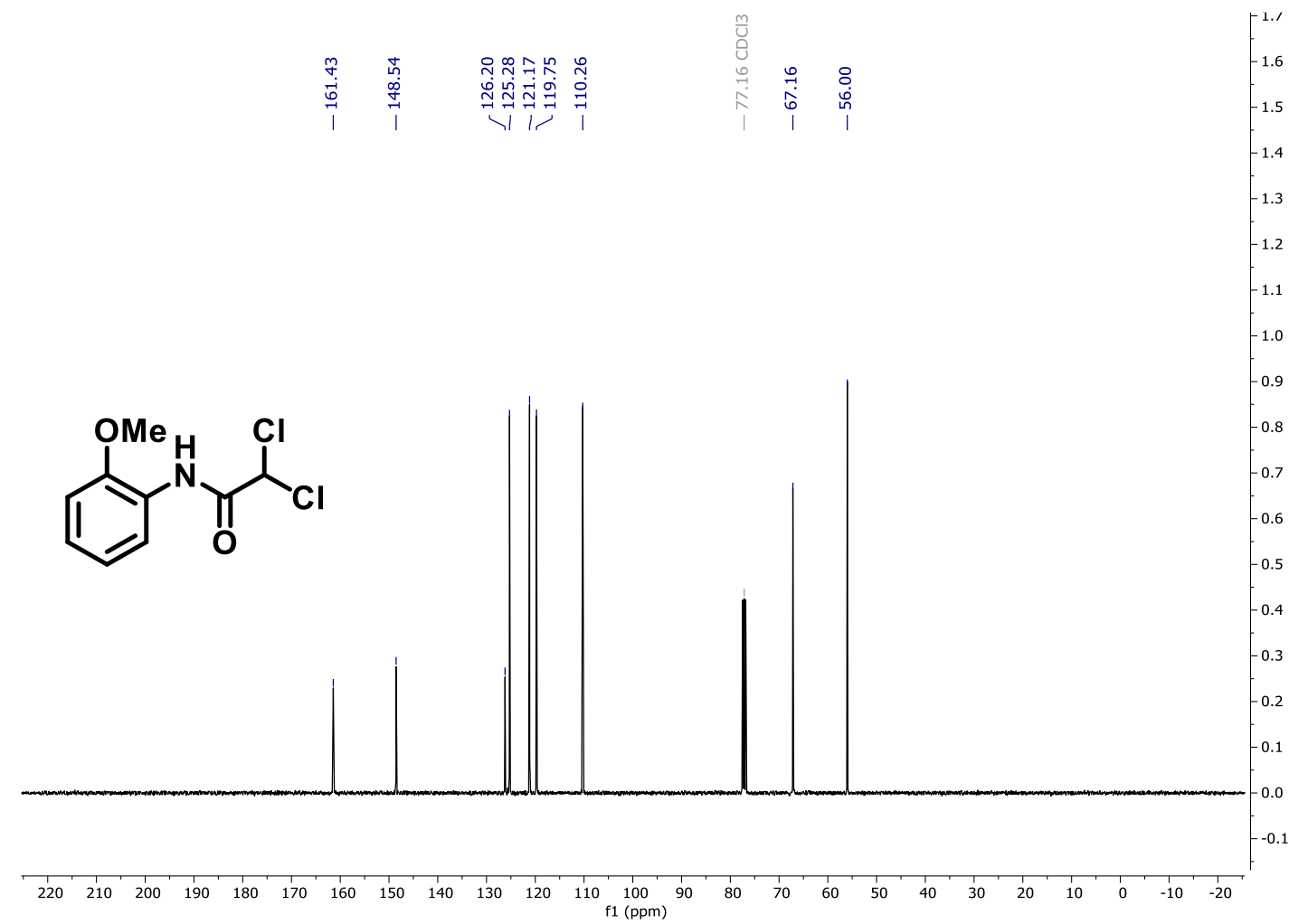


2,2-Dichloro- $N$-(2-methylphenyl)acetamide 3j

${ }^{1} \mathrm{H} \mathrm{NMR}\left(400 \mathrm{MHz}, \mathrm{CDCl}_{3}\right)$

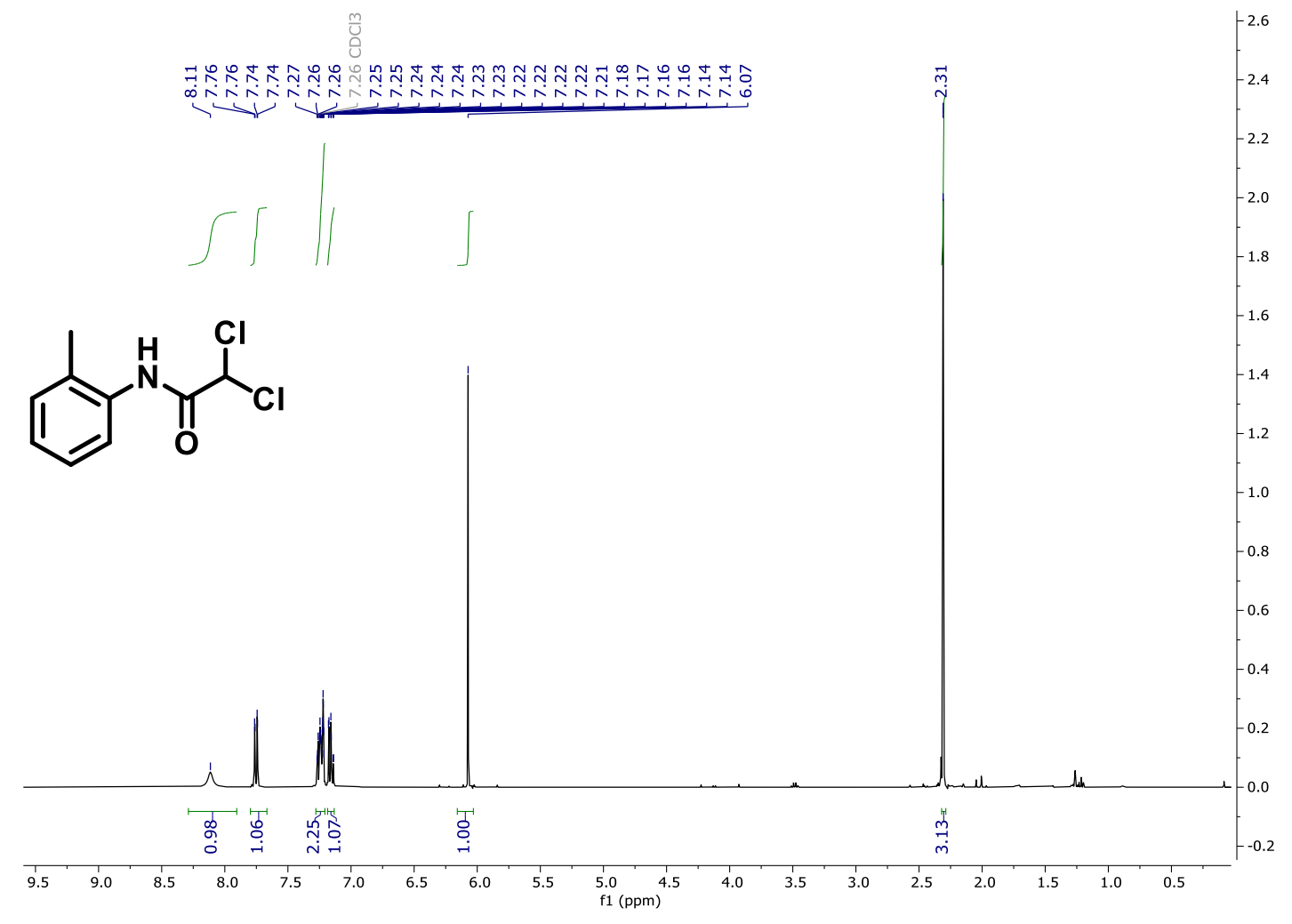


${ }^{13} \mathrm{C}$ NMR (101 MHz, $\mathrm{CDCl}_{3}$ )

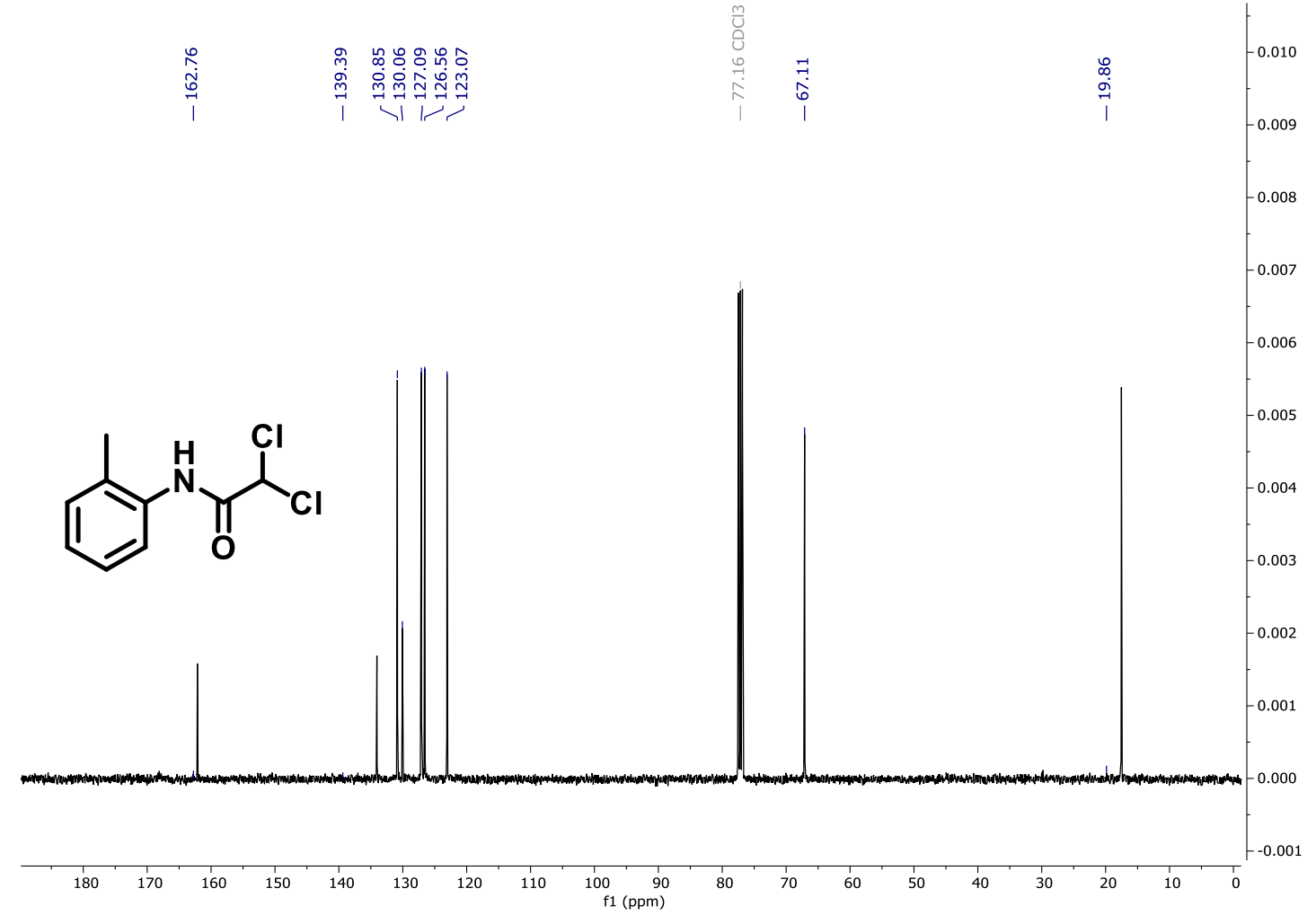


2,2-Dichloro- $N$-(2-ethylphenyl)acetamide 3k

${ }^{1} \mathrm{H}$ NMR (400 MHz, $\mathrm{CDCl}_{3}$ )

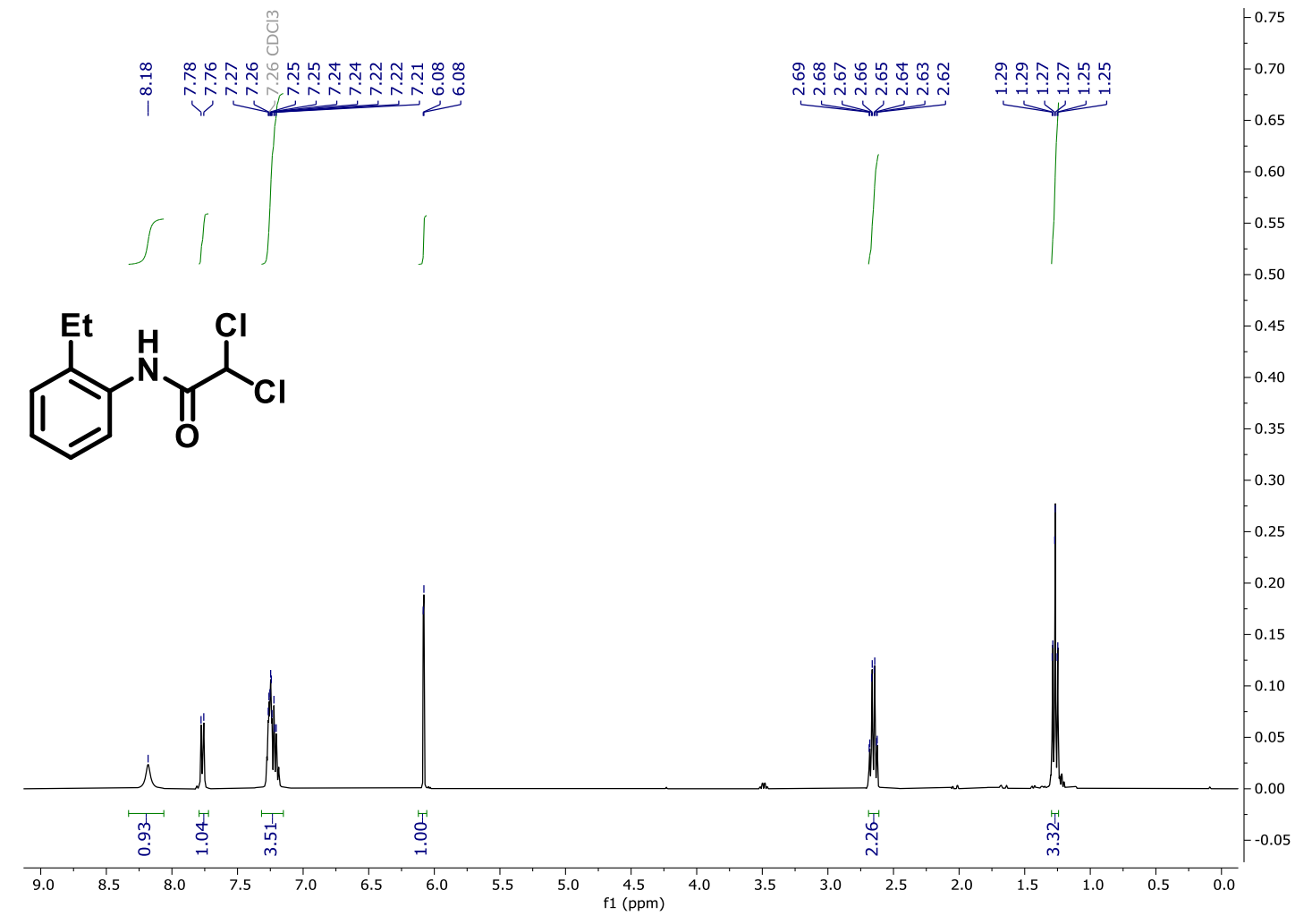


${ }^{13} \mathrm{C}$ NMR (101 MHz, $\left.\mathrm{CDCl}_{3}\right)$

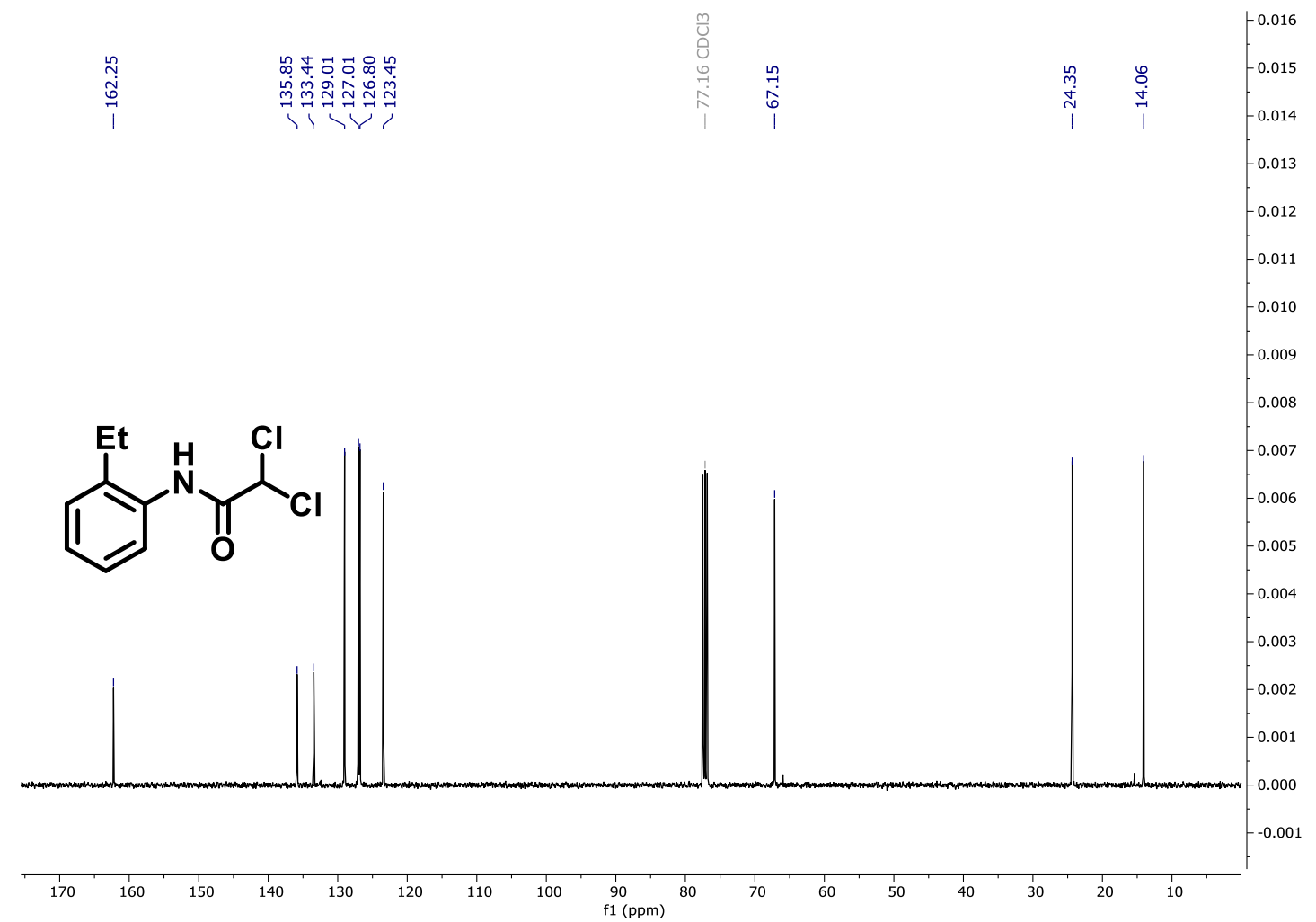


2,2-Dichloro-N-(2-(4,4,5,5-tetramethyl-1,3,2-dioxaborolan-2-yl)phenyl)acetamide 3I

${ }^{1} \mathrm{H}$ NMR (400 MHz, $\mathrm{CDCl}_{3}$ )

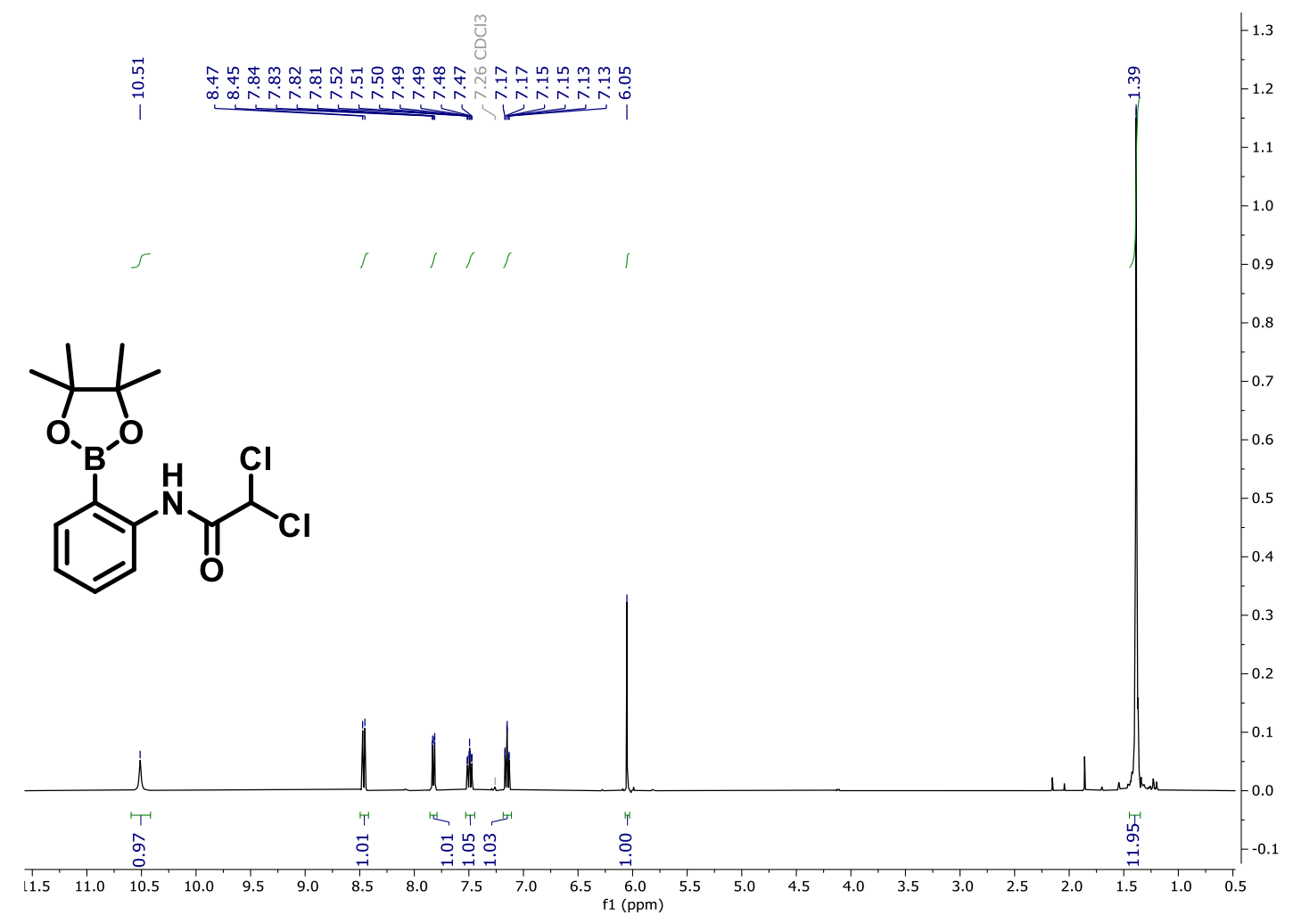


${ }^{13} \mathrm{C}$ NMR (101 MHz, $\left.\mathrm{CDCl}_{3}\right)$

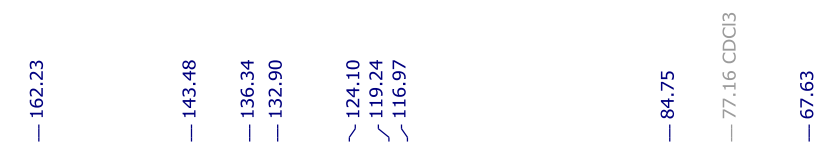

$\prod_{0.0}^{1}$

$\underbrace{\text { I }}_{0}$

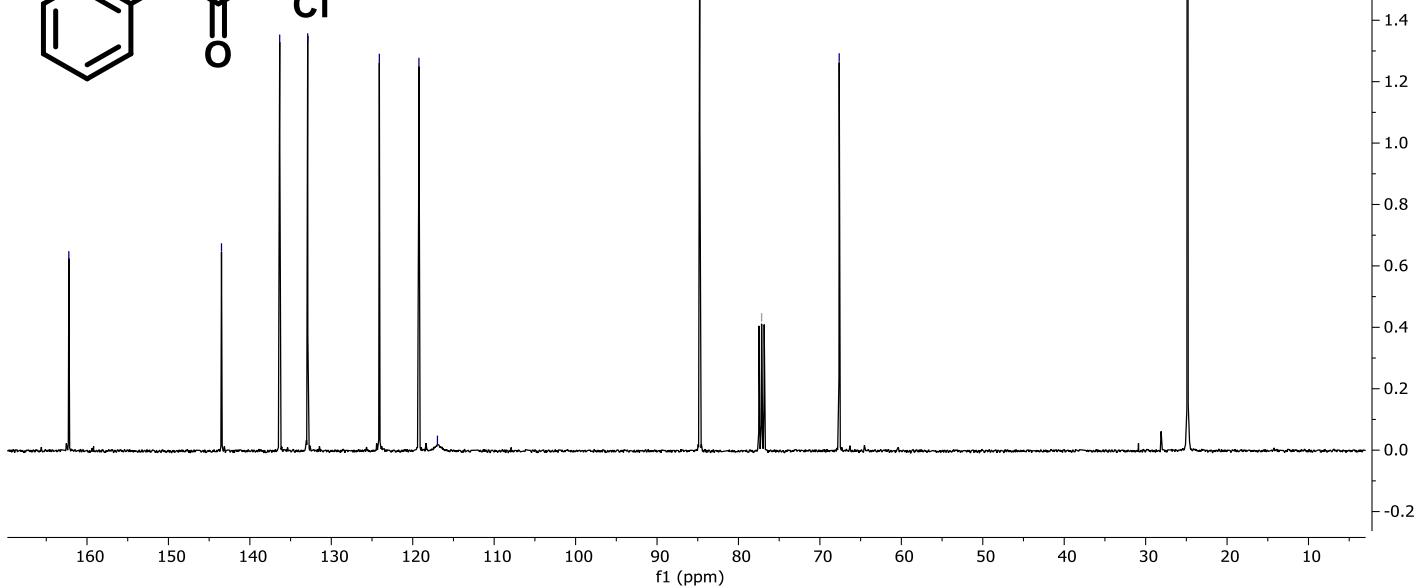


2,2-Dichloro- $N$-(naphthalen-1-yl)acetamide 3m

${ }^{1} \mathrm{H}$ NMR (400 MHz, $\left.\mathrm{CDCl}_{3}\right)$

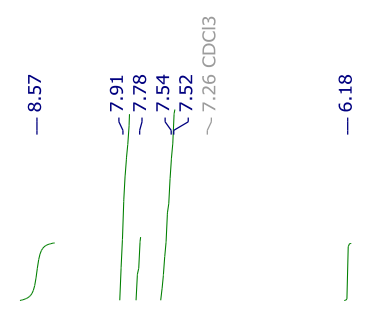

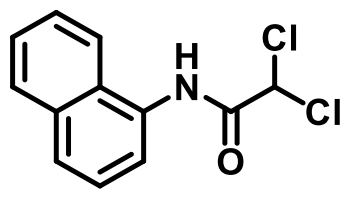

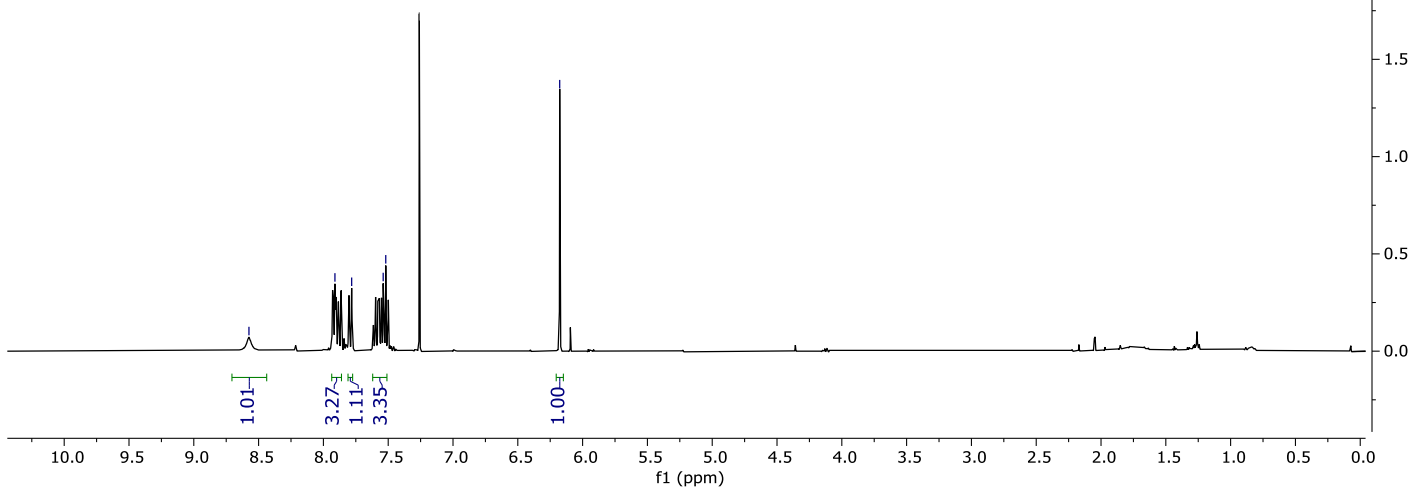


${ }^{13} \mathrm{C}$ NMR (101 MHz, $\left.\mathrm{CDCl}_{3}\right)$

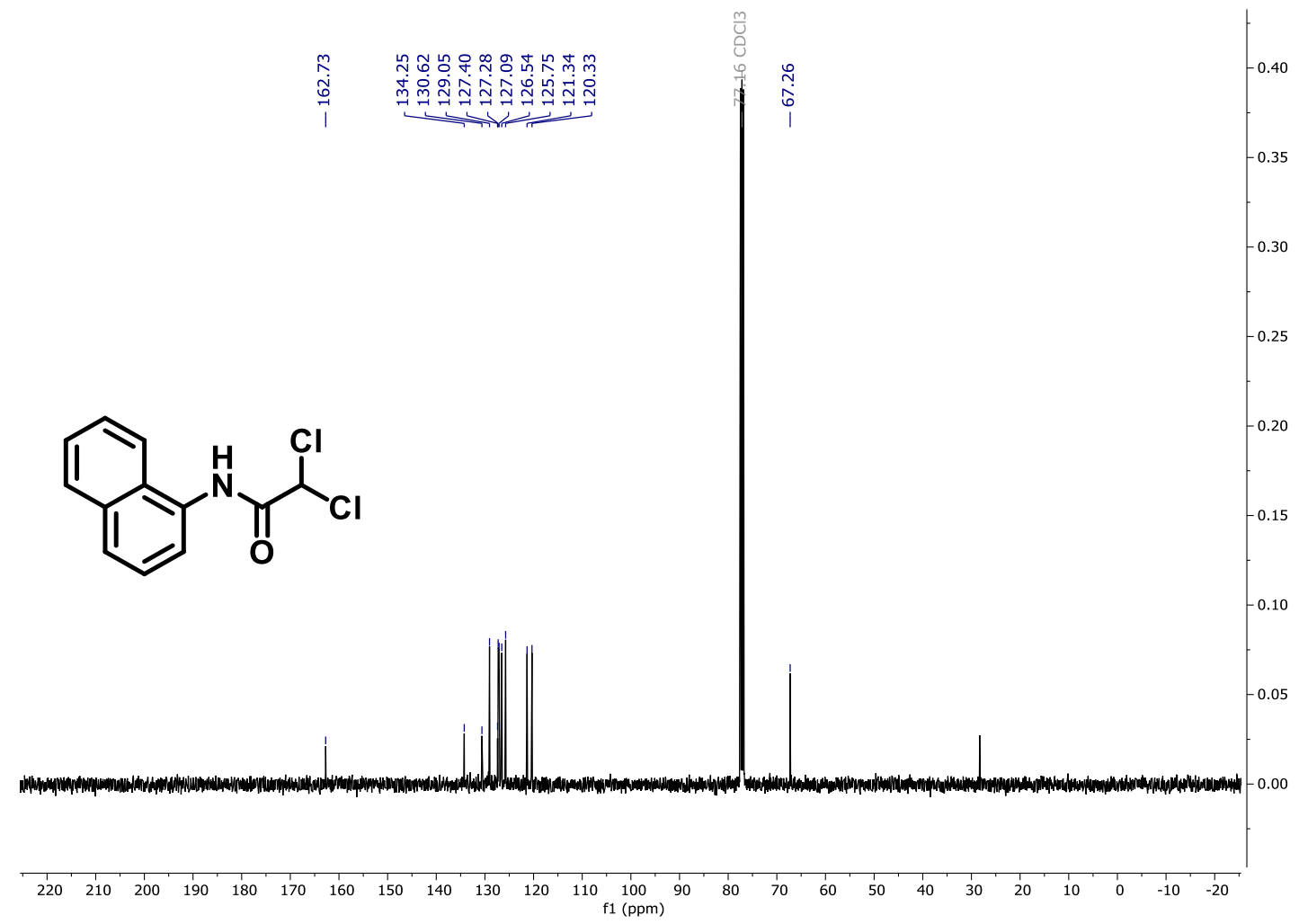


2,2-Dichloro-N-(2-methyl-4-fluorophenyl)acetamide 3n

${ }^{1} \mathrm{H}$ NMR $\left(400 \mathrm{MHz}, \mathrm{CDCl}_{3}\right)$

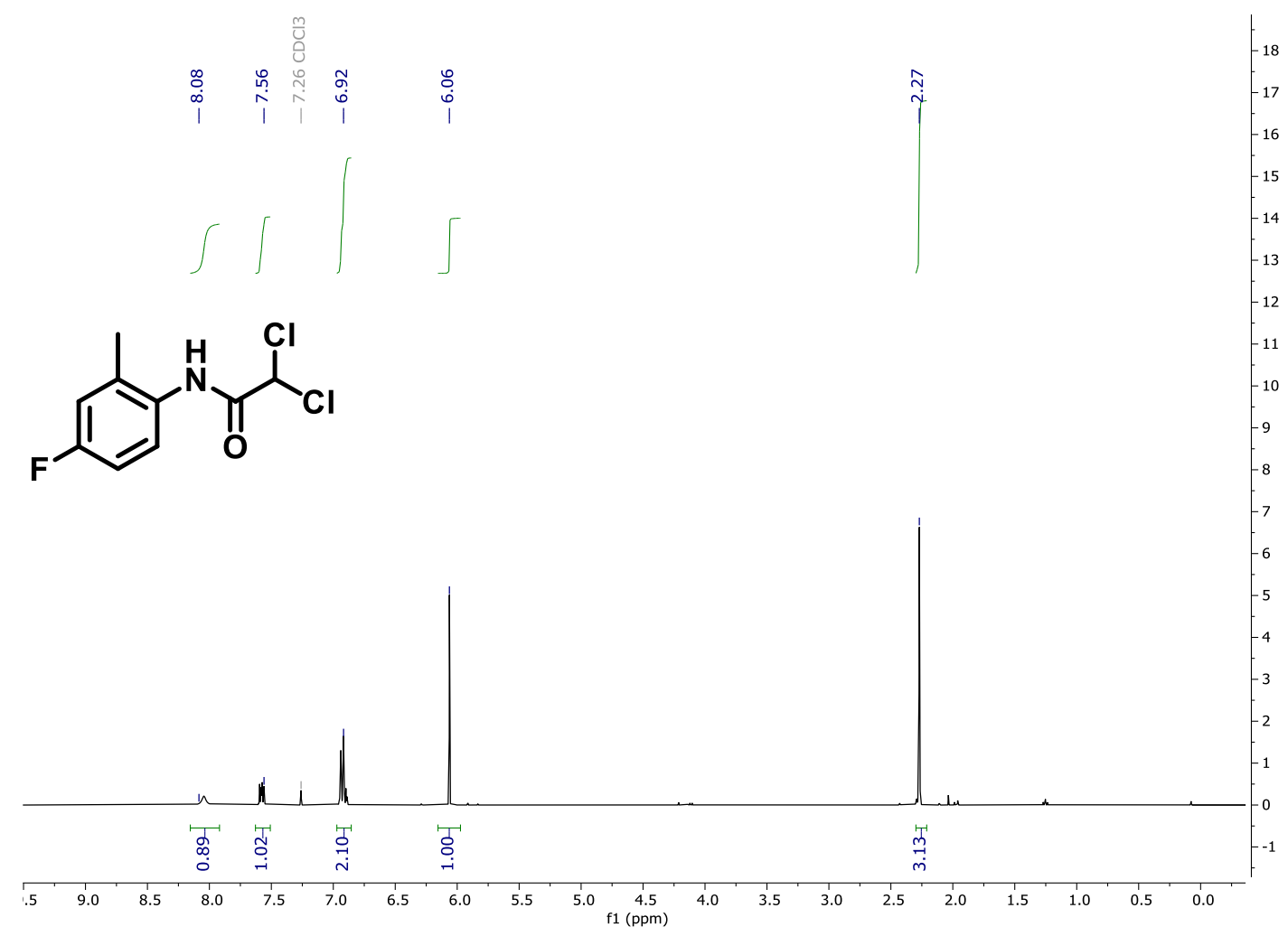


${ }^{13} \mathrm{C}$ NMR (101 MHz, $\left.\mathrm{CDCl}_{3}\right)$

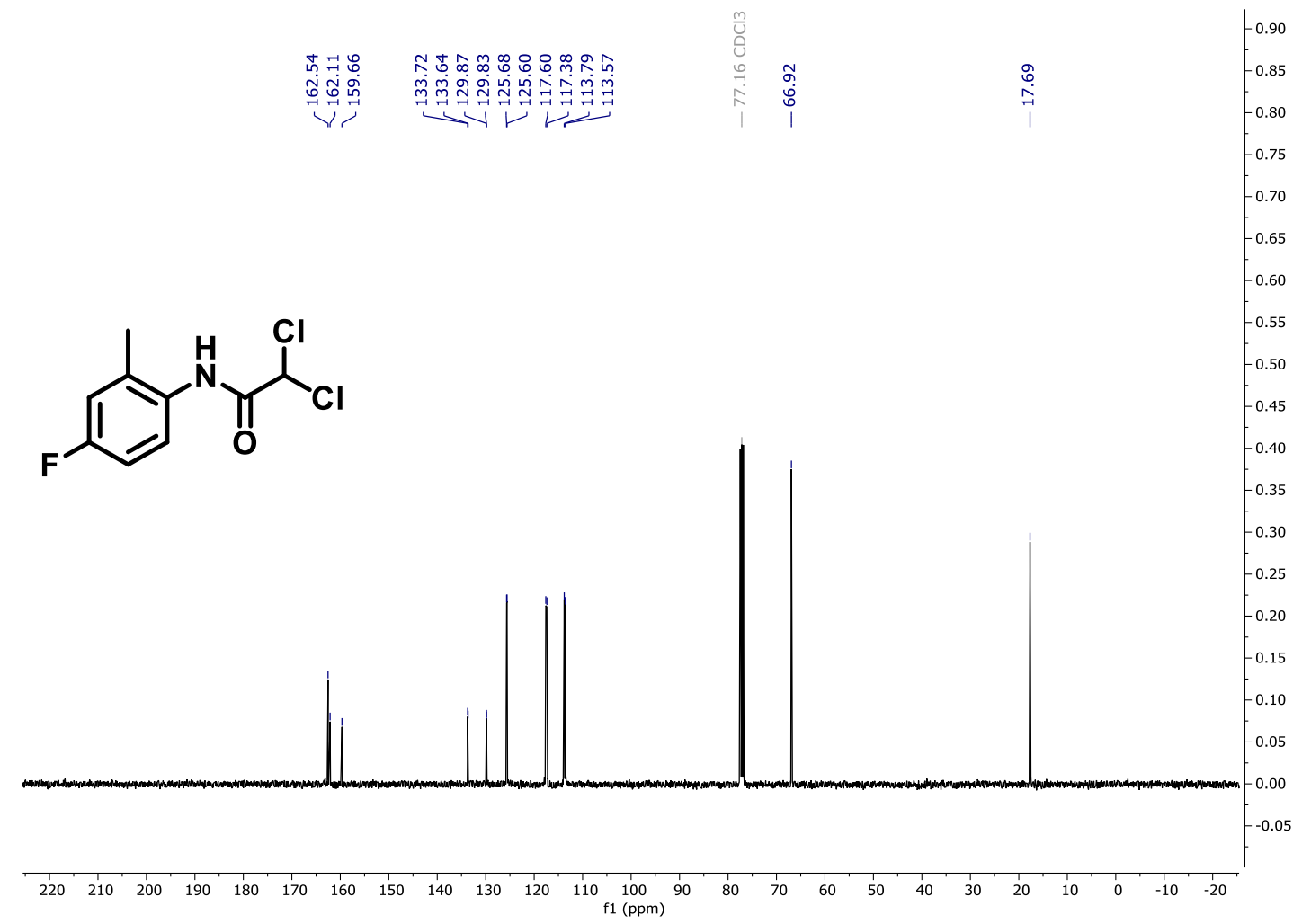


${ }^{19} \mathrm{~F}$ NMR (376 MHz, $\mathrm{CDCl}_{3}$ )

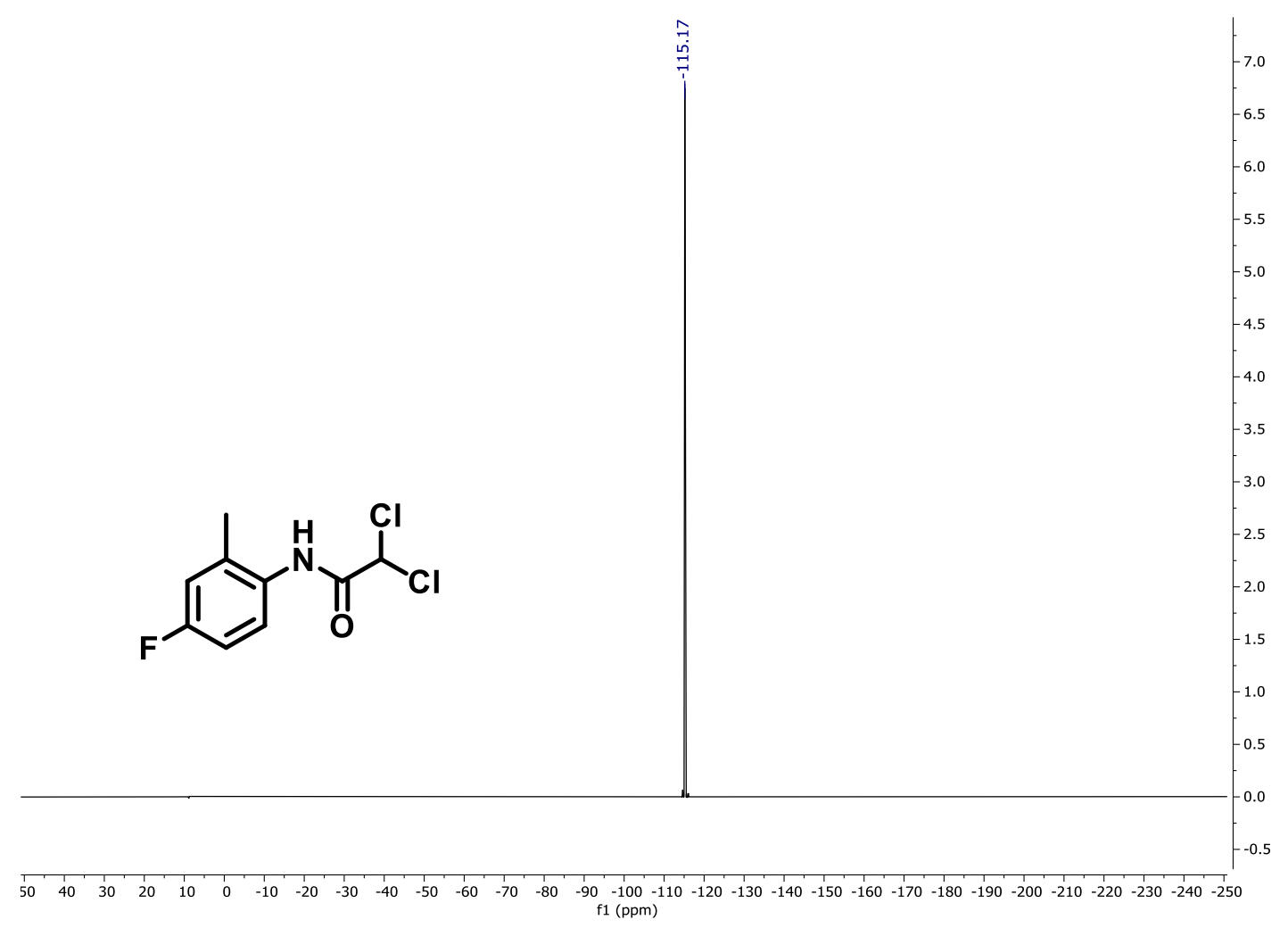


2,2-Dichloro- $N$-(2,6-dimethylphenyl)acetamide 30

${ }^{1} \mathrm{H}$ NMR (400 MHz, $\mathrm{CDCl}_{3}$ )

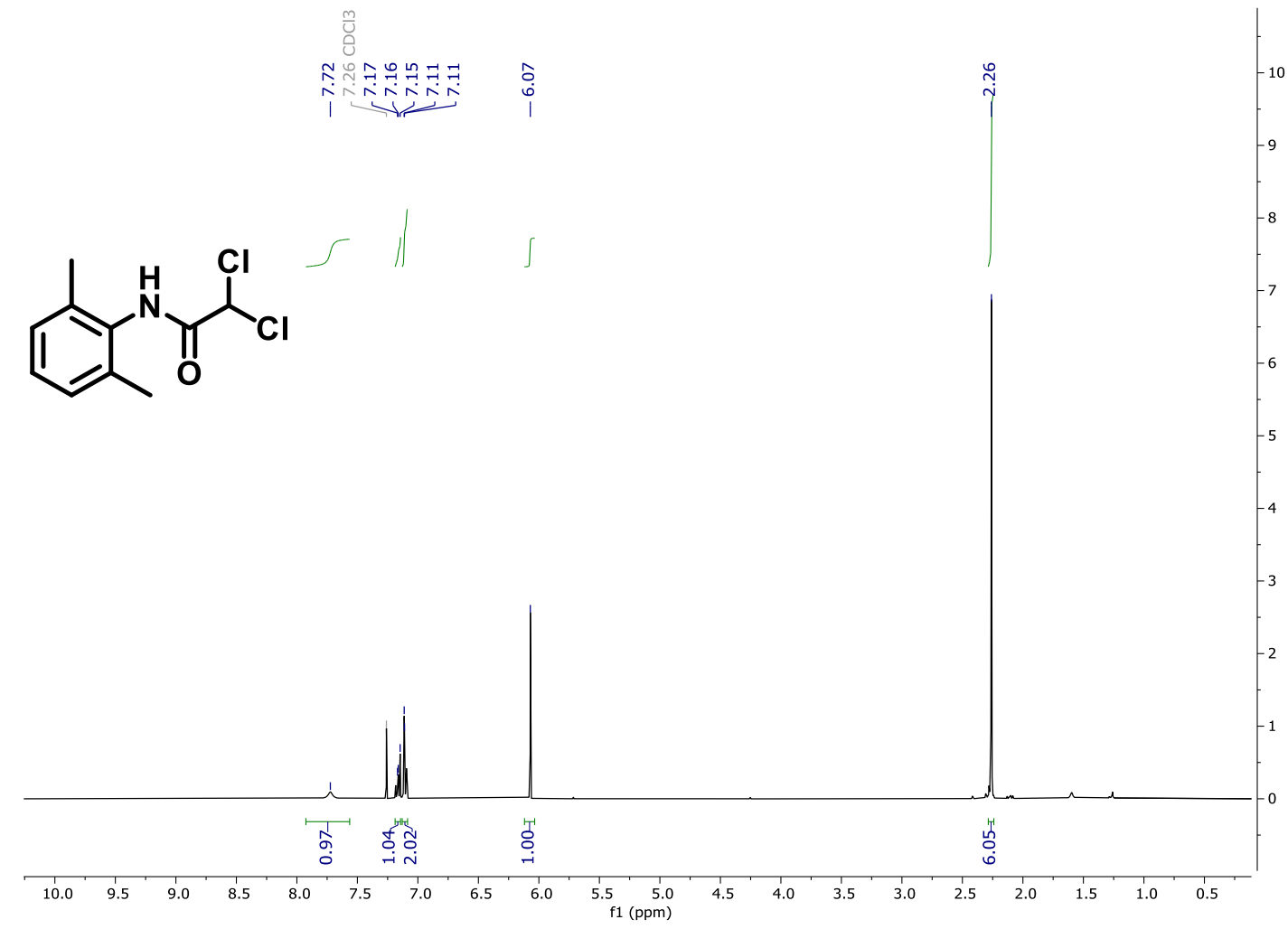


${ }^{13} \mathrm{C}$ NMR (101 MHz, $\mathrm{CDCl}_{3}$ )

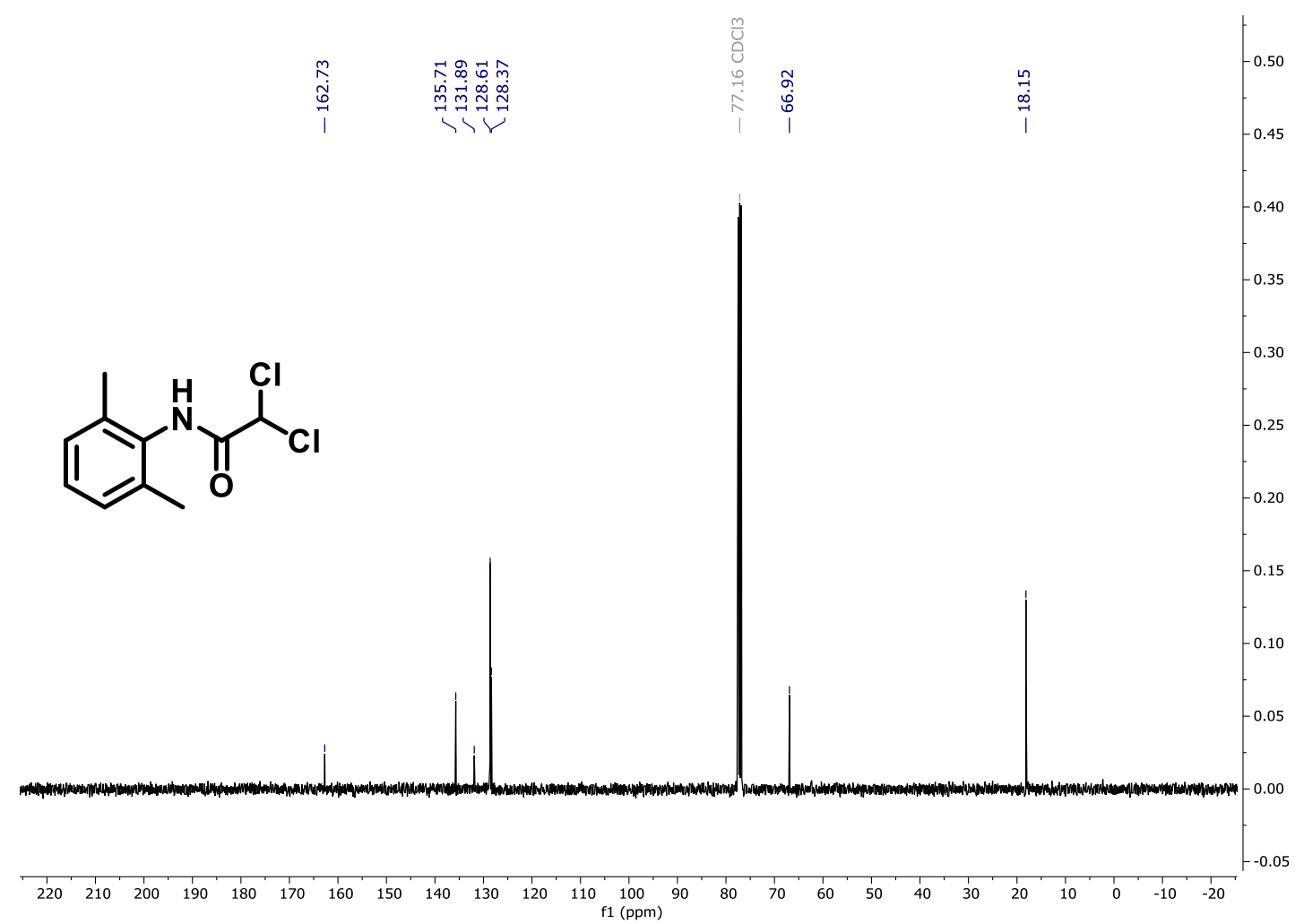


4-(2,2-Dichloroacetamido)benzoic acid 3p

${ }^{1} \mathrm{H}$ NMR $\left(400 \mathrm{MHz}, \mathrm{CD}_{3} \mathrm{OD}\right)$

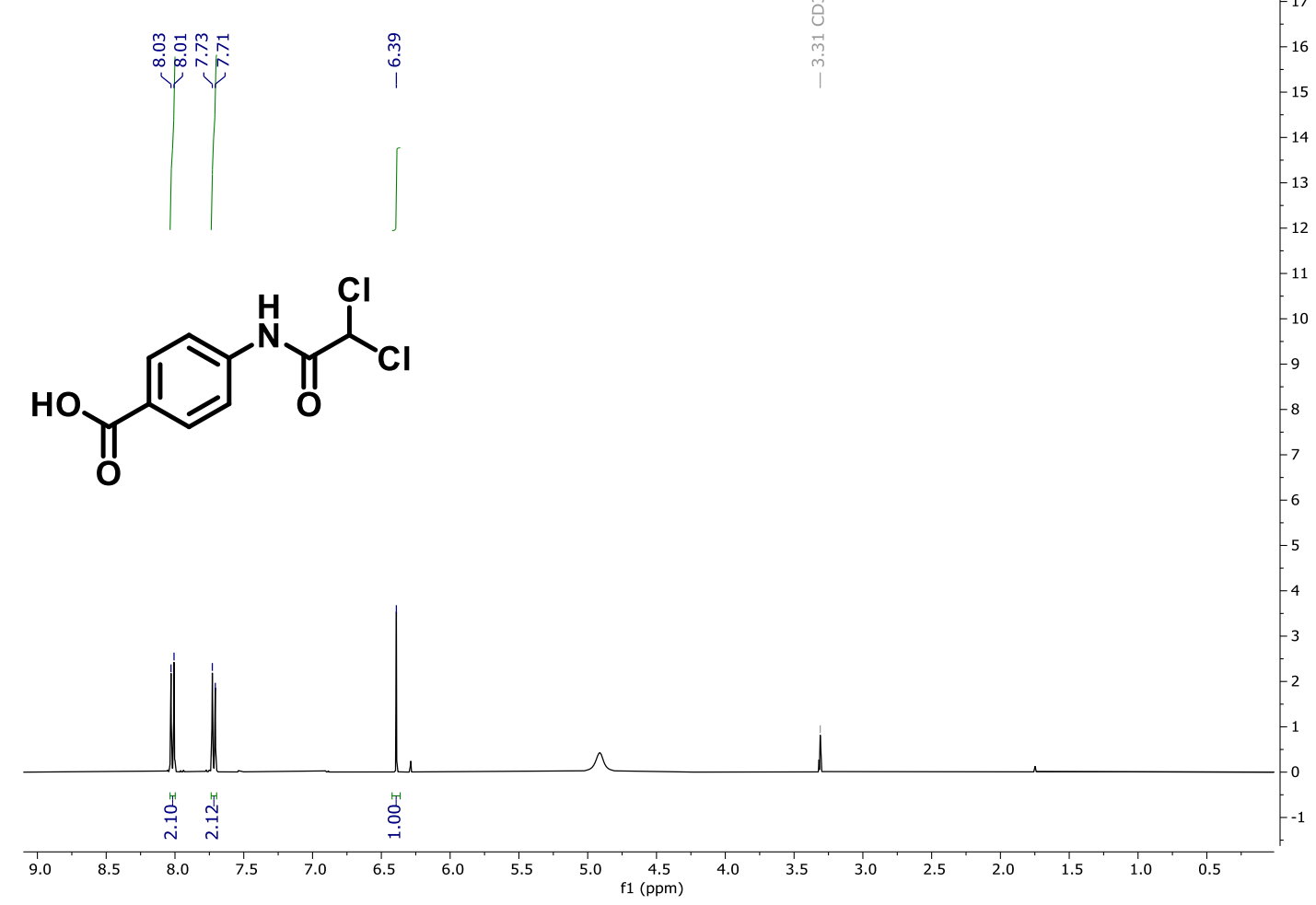


${ }^{13} \mathrm{C}$ NMR (101 MHz, CD ${ }_{3} \mathrm{OD}$ )

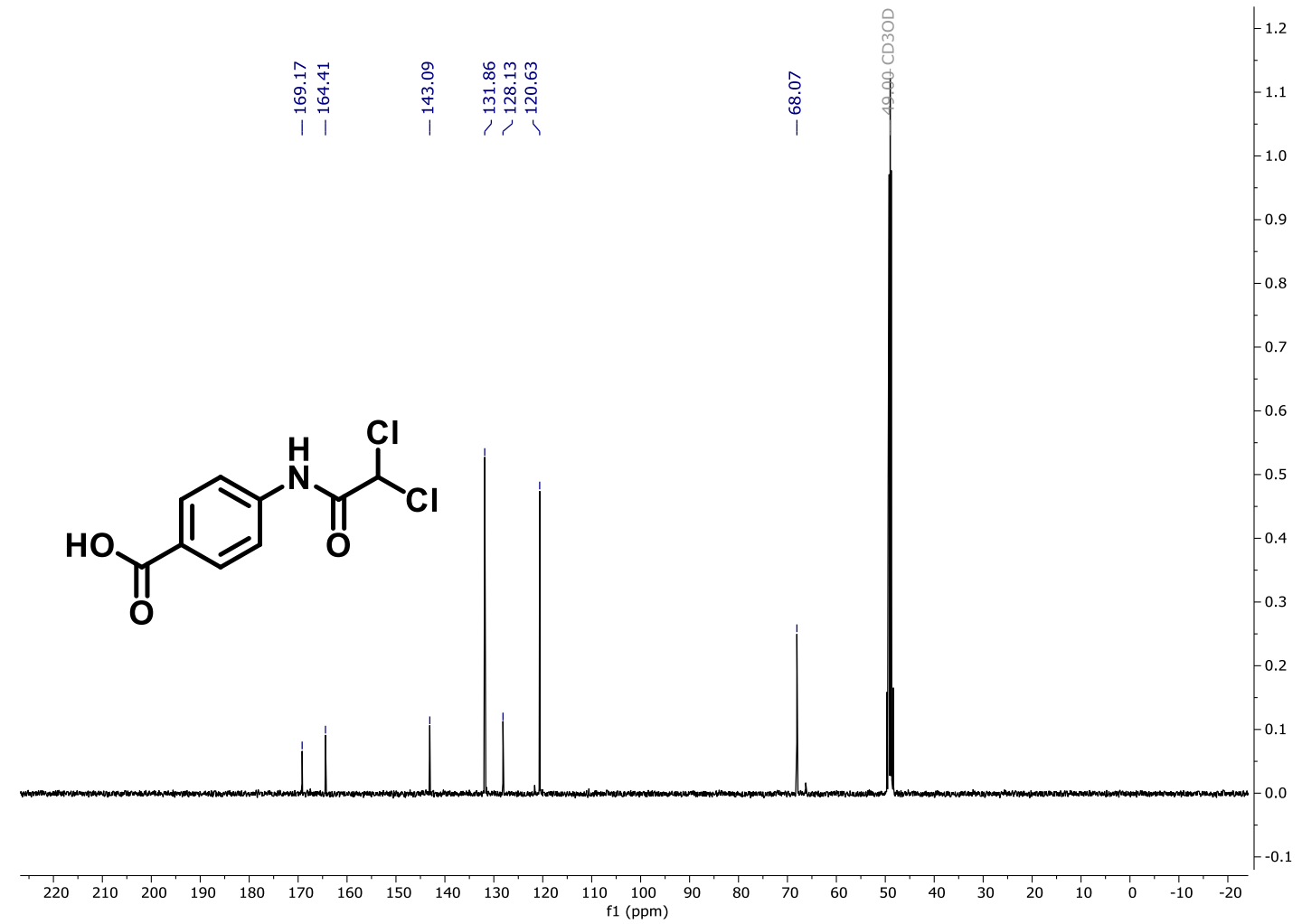


${ }^{1} \mathrm{H}$ NMR $\left(400 \mathrm{MHz},\left(\mathrm{CD}_{3}\right)_{2} \mathrm{SO}\right)$

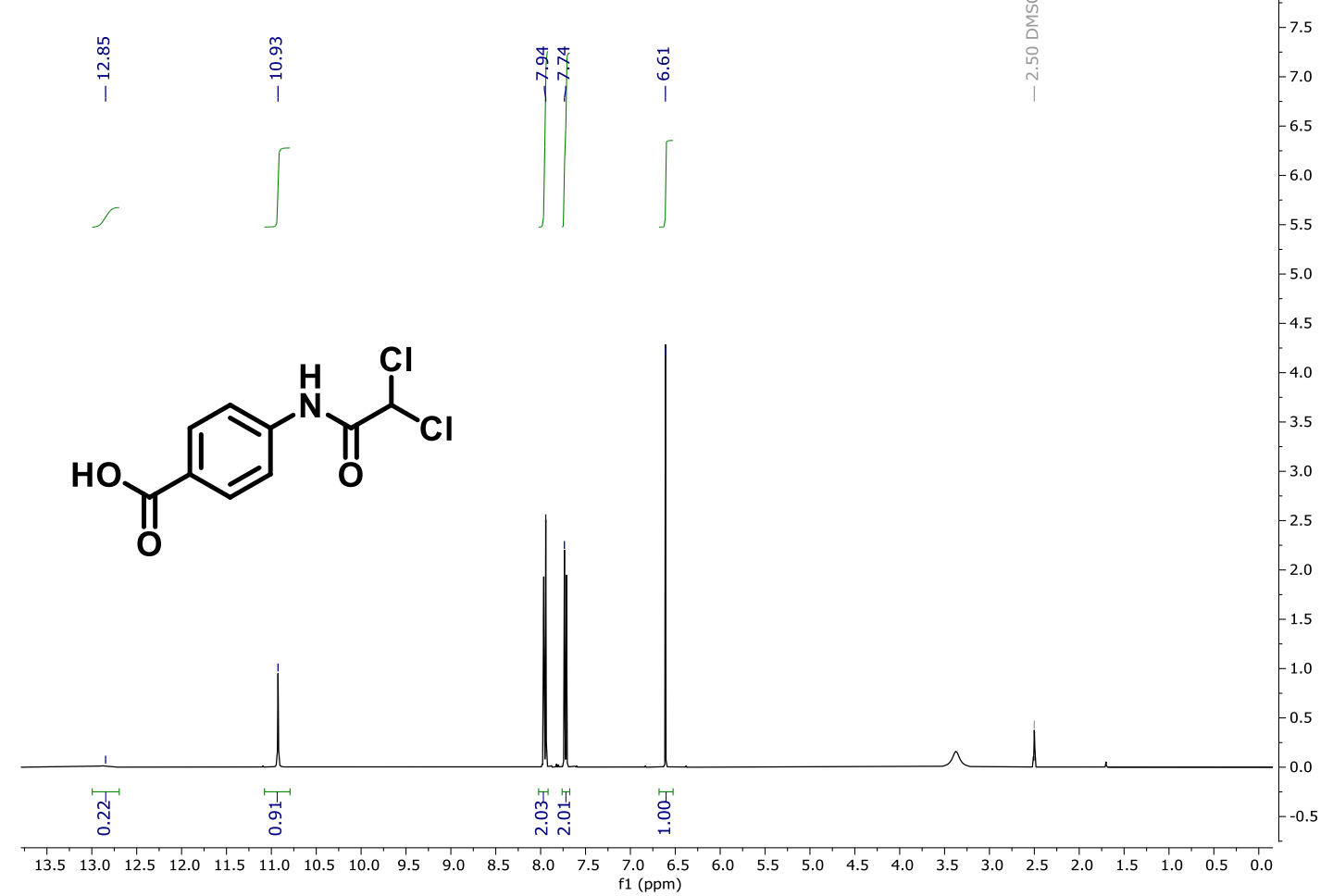


${ }^{13} \mathrm{C}$ NMR (101 MHz, $\left.\left(\mathrm{CD}_{3}\right)_{2} \mathrm{SO}\right)$

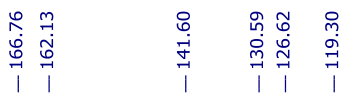

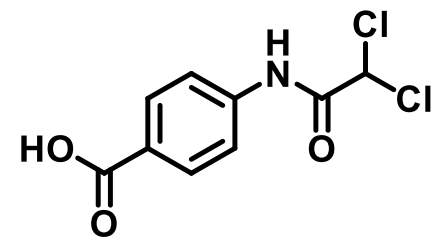

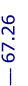

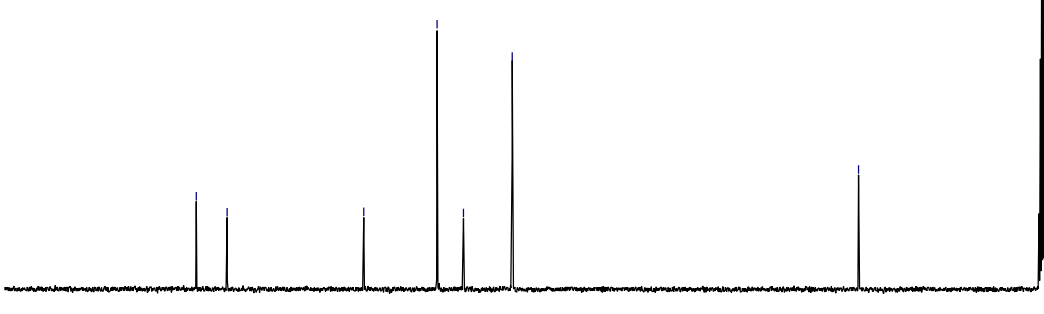

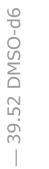

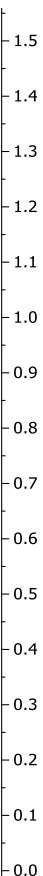

$\begin{array}{lllllllllll}190 & 180 & 170 & 160 & 150 & 140 & 130 & 120 & 110 & \begin{array}{c}100 \\ \mathrm{f} 1(\mathrm{ppm})\end{array}\end{array}$ 
4-(4-(2,2-Dichloroacetamido)phenyl)butanoic acid 3q

${ }^{1} \mathrm{H}$ NMR (400 MHz, $\left.\mathrm{CD}_{3} \mathrm{OD}\right)$

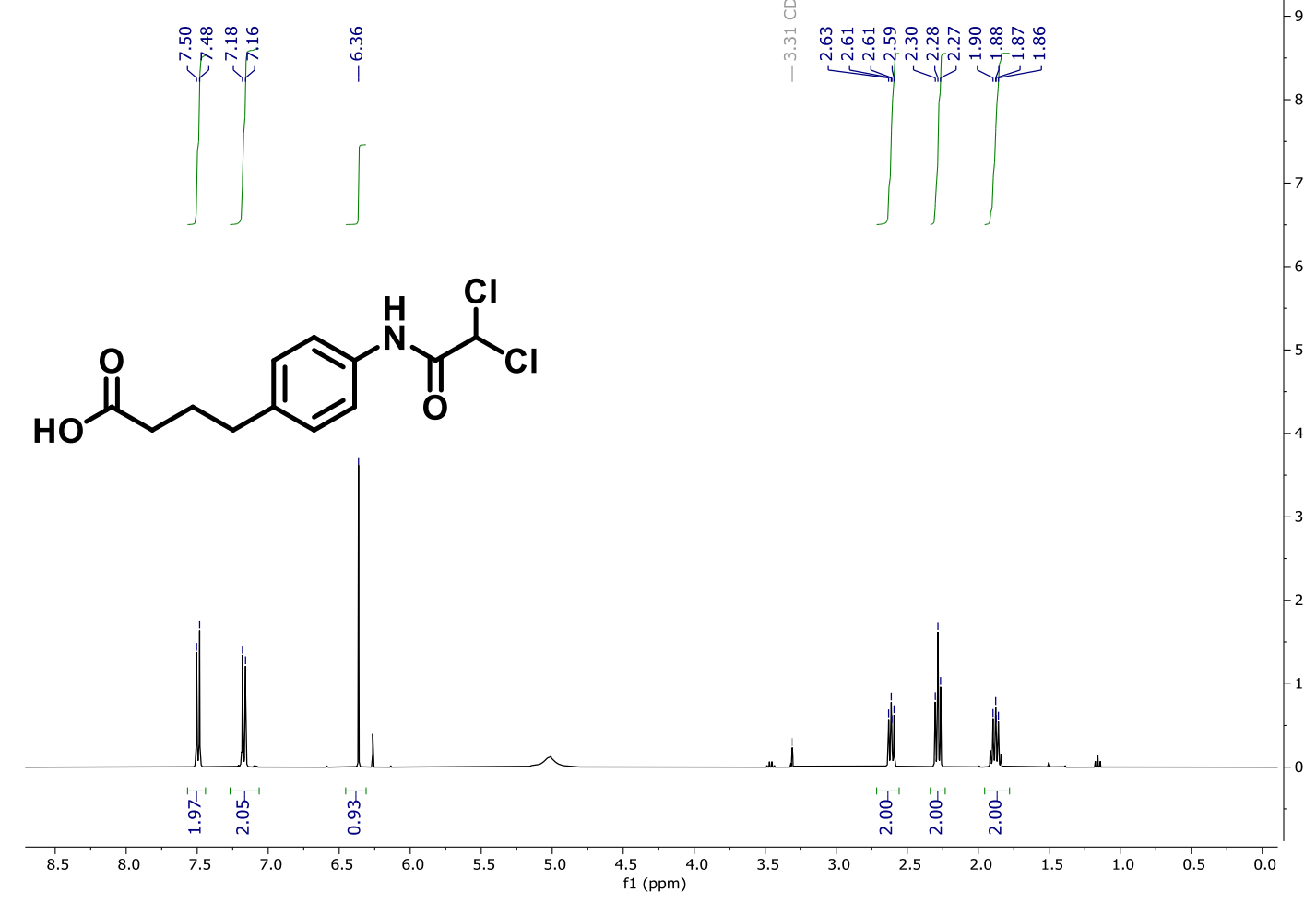


${ }^{13} \mathrm{C}$ NMR (101 MHz, $\left.\mathrm{CD}_{3} \mathrm{OD}\right)$

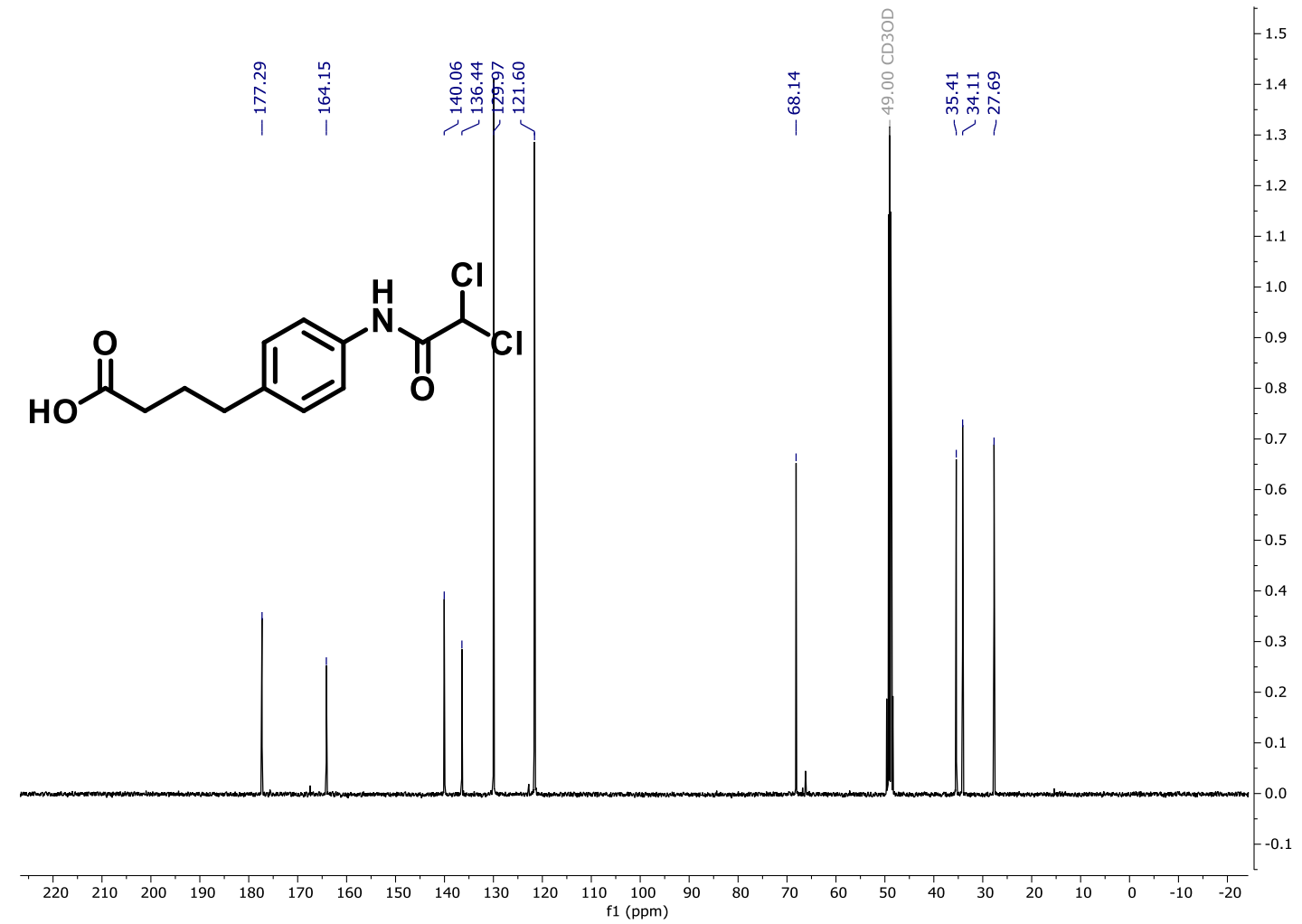


$\mathrm{N}$-(4-Methoxybenzyl)-2,2-dichloroacetamide 3r

${ }^{1} \mathrm{H}$ NMR (400 MHz, $\left.\mathrm{CDCl}_{3}\right)$

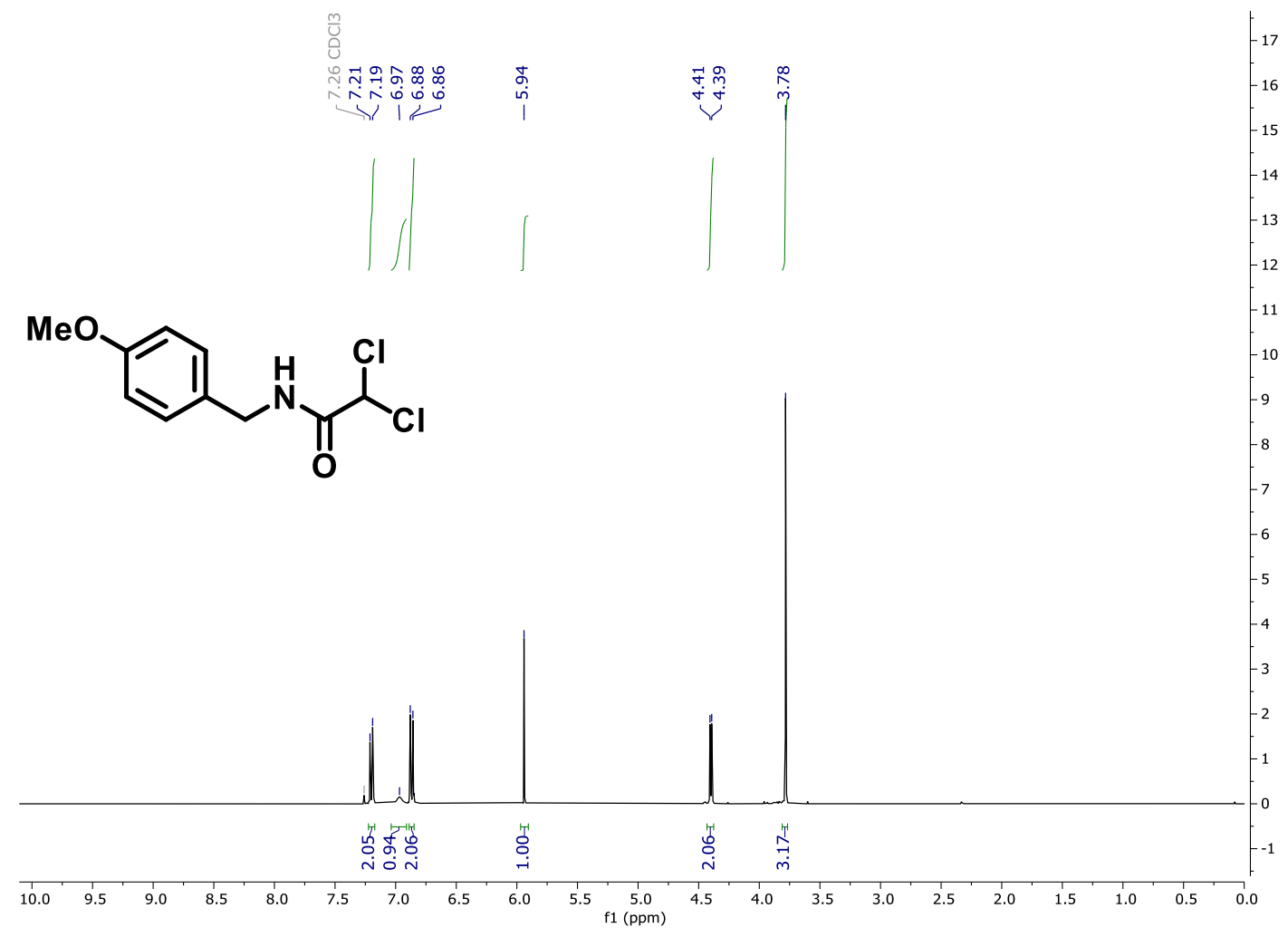


${ }^{13} \mathrm{C}$ NMR (101 MHz, $\left.\mathrm{CDCl}_{3}\right)$

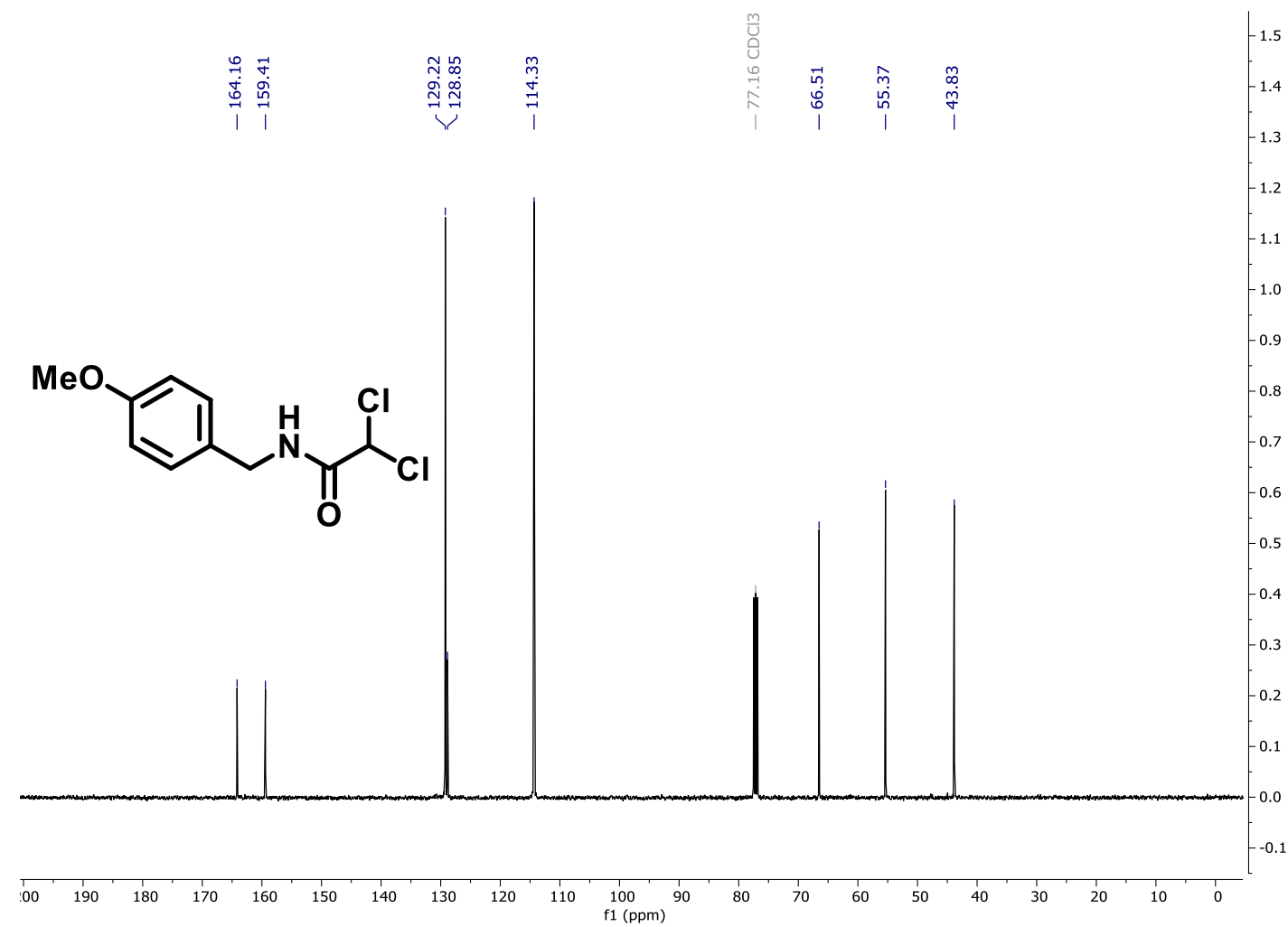


$\mathrm{N}$-(4-Methylbenzyl)-2,2-dichloroacetamide 3s

${ }^{1} \mathrm{H}$ NMR $\left(400 \mathrm{MHz}, \mathrm{CDCl}_{3}\right)$

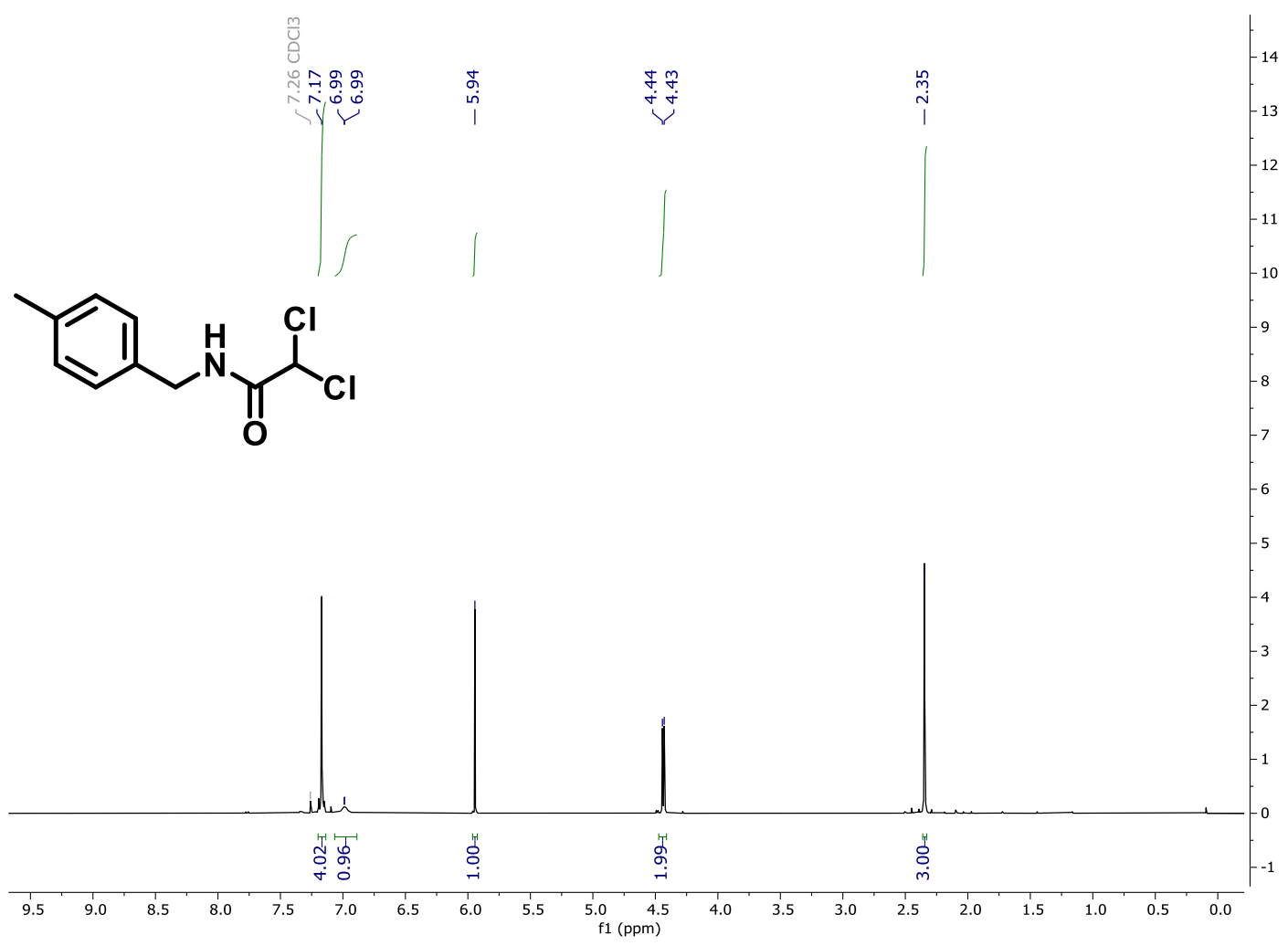


${ }^{13} \mathrm{C}$ NMR (101 MHz, $\left.\mathrm{CDCl}_{3}\right)$

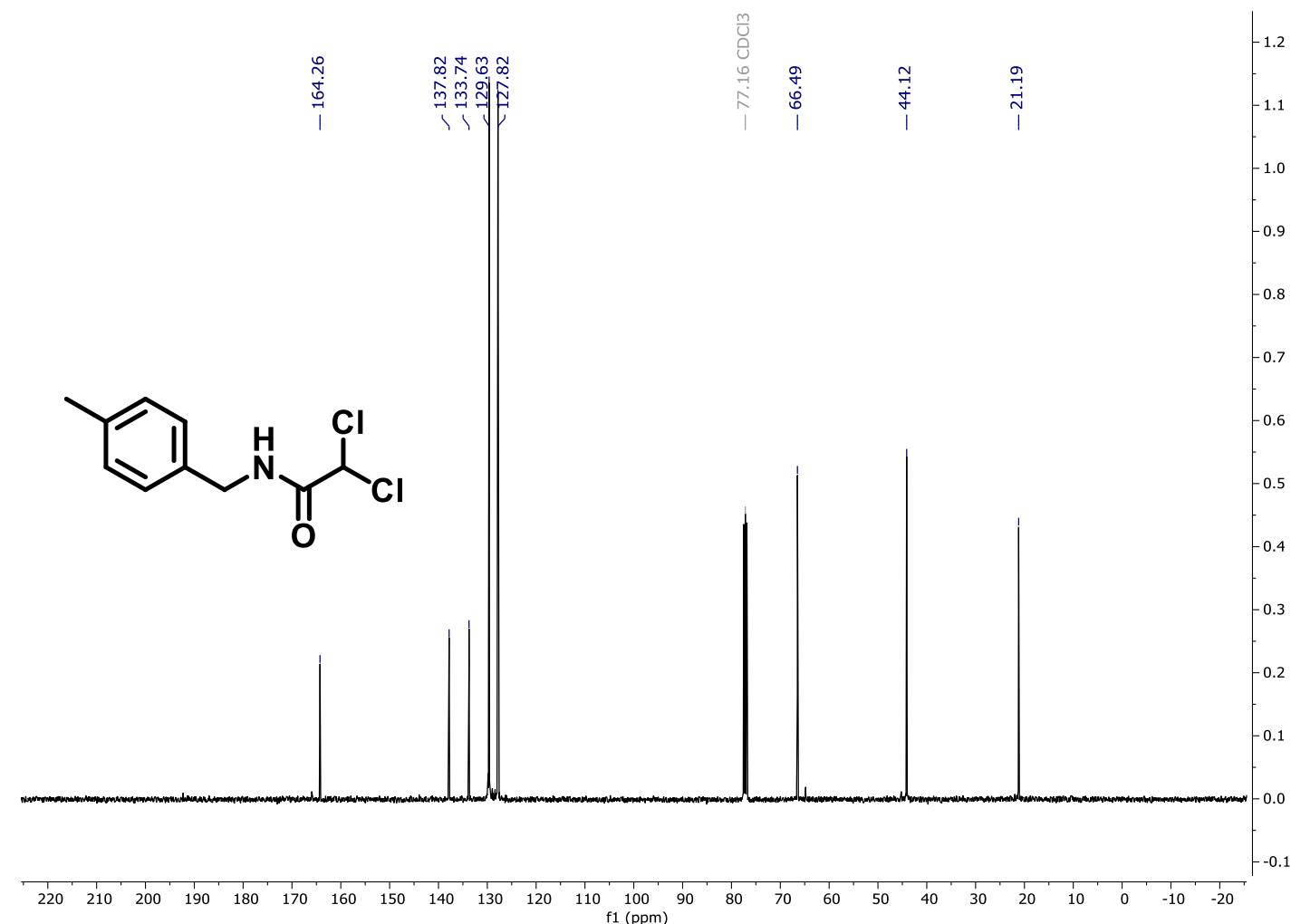


$\mathrm{N}$-(4-Trifluoromethylbenzyl)-2,2-dichloroacetamide 3t

${ }^{1} \mathrm{H} \mathrm{NMR}\left(400 \mathrm{MHz}, \mathrm{CDCl}_{3}\right)$

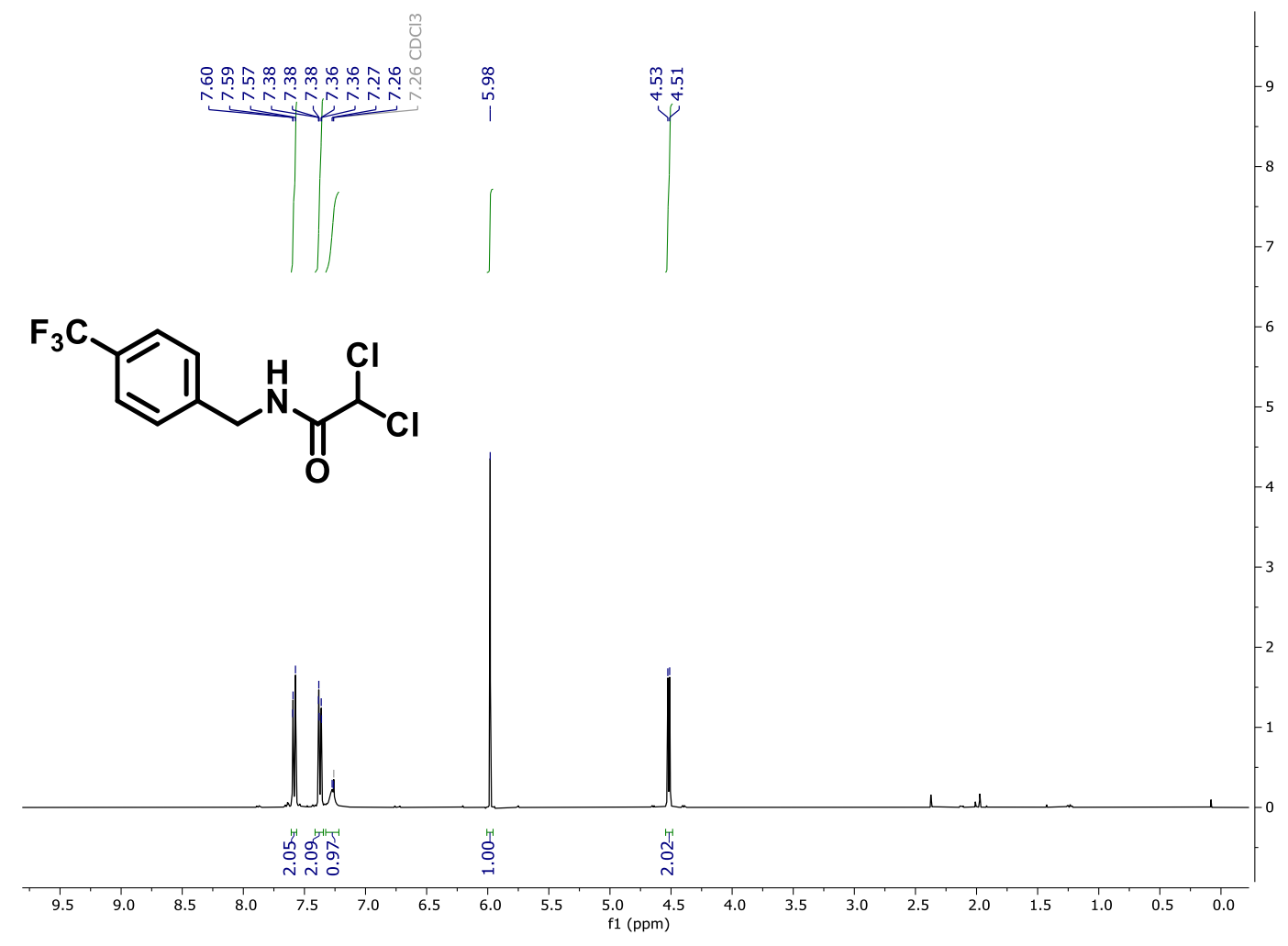


${ }^{13} \mathrm{C}$ NMR (101 MHz, $\mathrm{CDCl}_{3}$ )

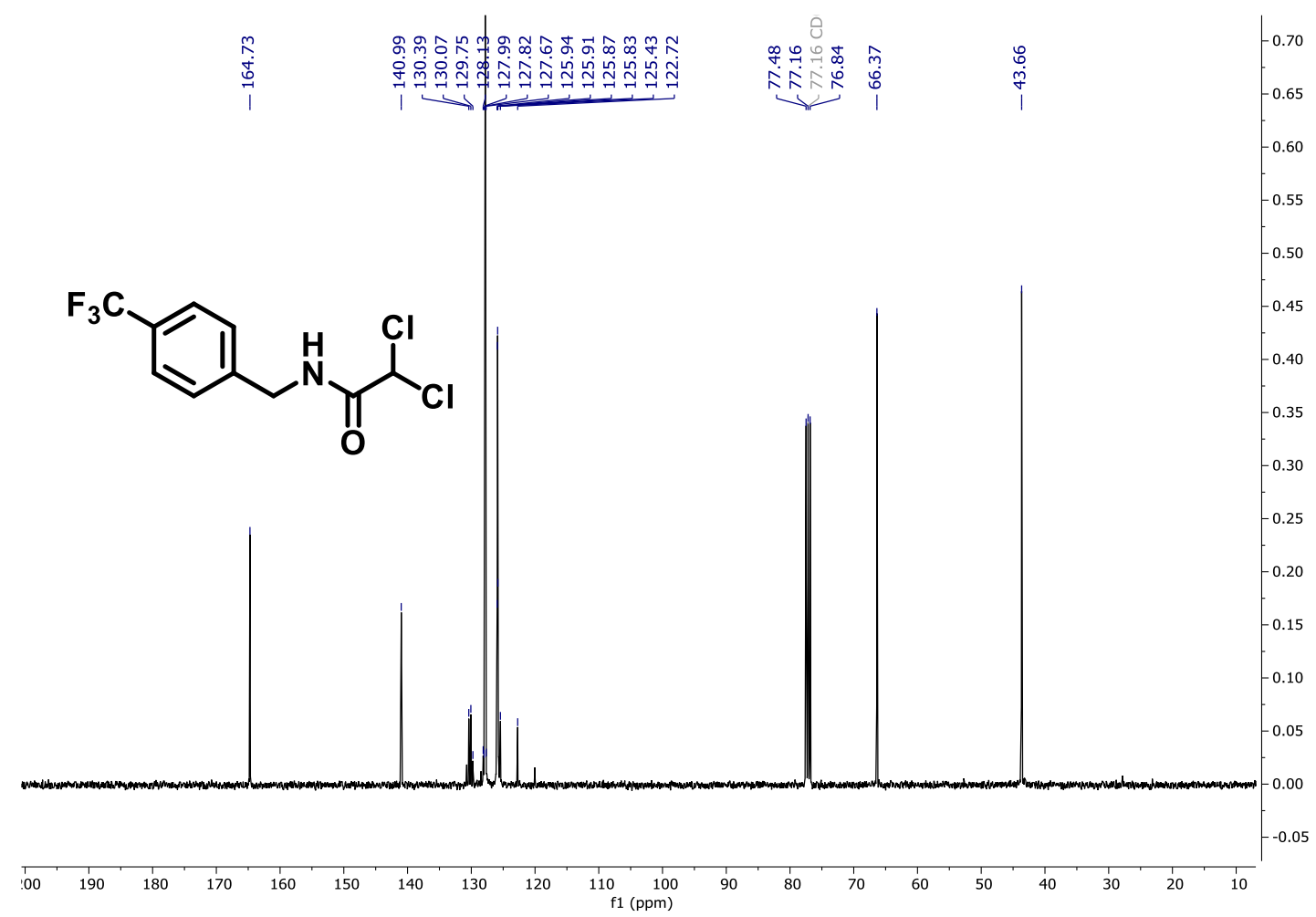


${ }^{19} \mathrm{~F}$ NMR (376 MHz, $\mathrm{CDCl}_{3}$ )

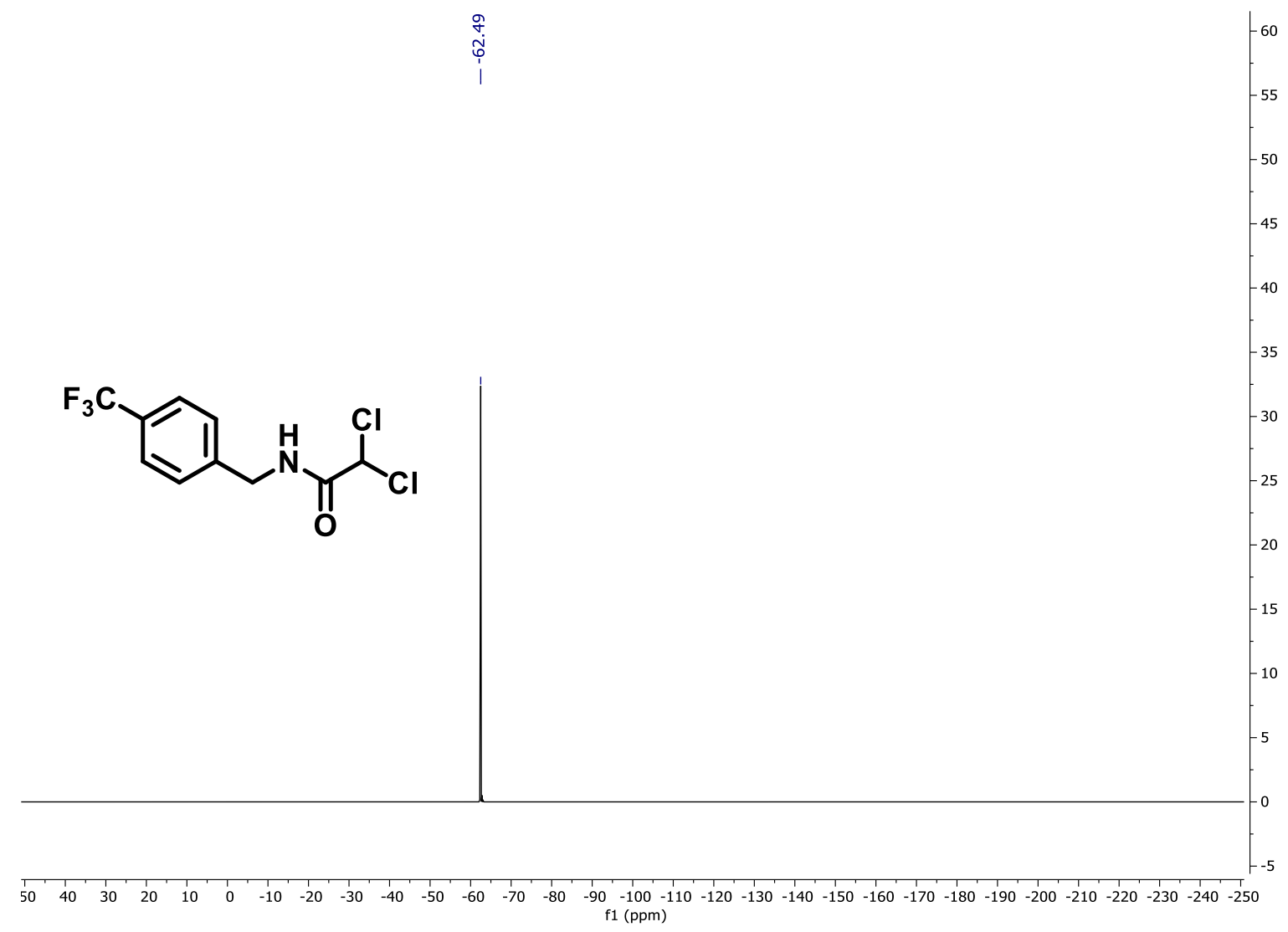


N-Benzyl-2,2-dichloroacetamide 3u

${ }^{1} \mathrm{H}$ NMR (400 MHz, $\mathrm{CDCl}_{3}$ )<smiles>O=C(NCc1ccccc1)C(Cl)Cl</smiles>
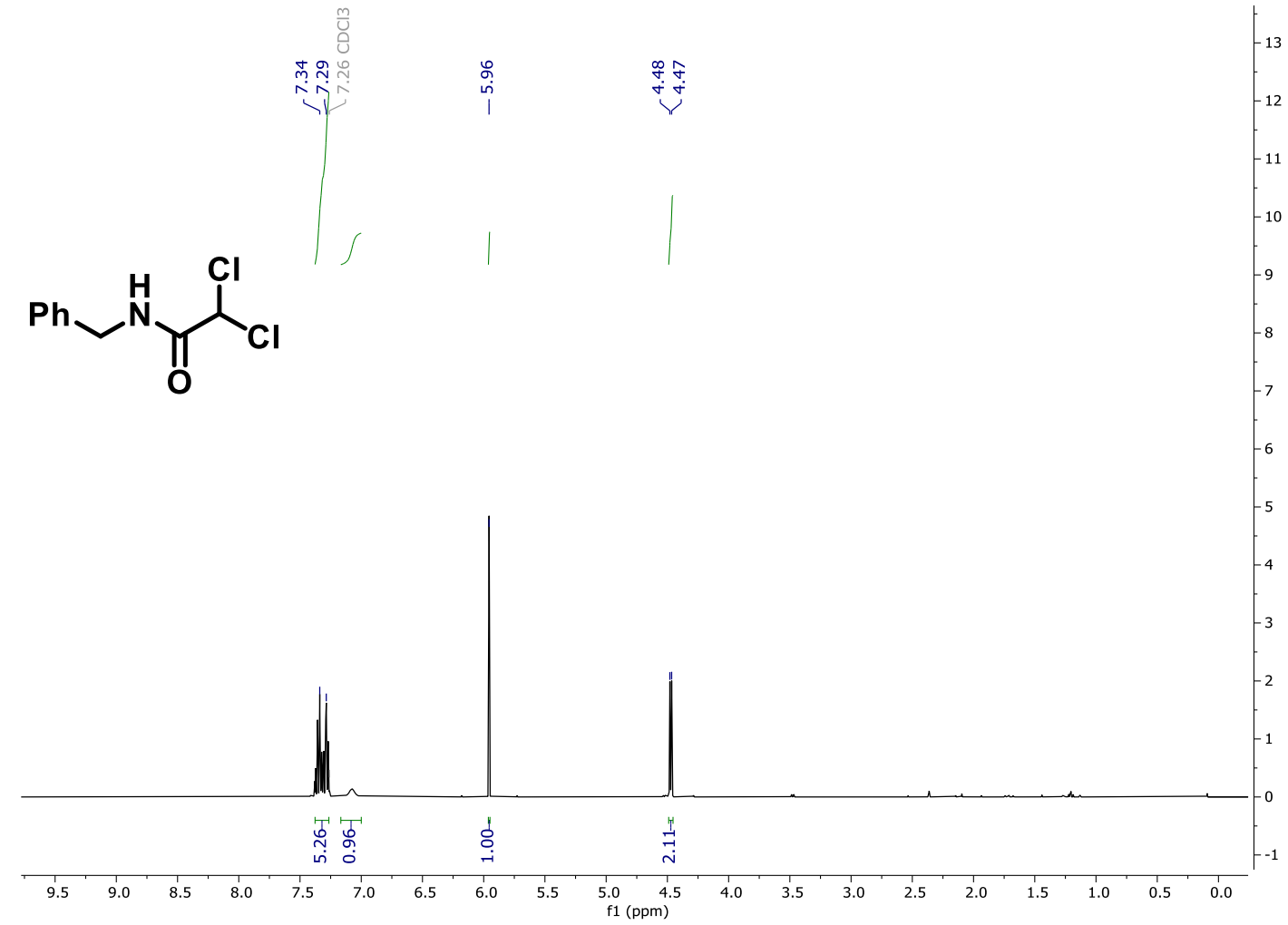
${ }^{13} \mathrm{C}$ NMR (101 MHz, $\left.\mathrm{CDCl}_{3}\right)$

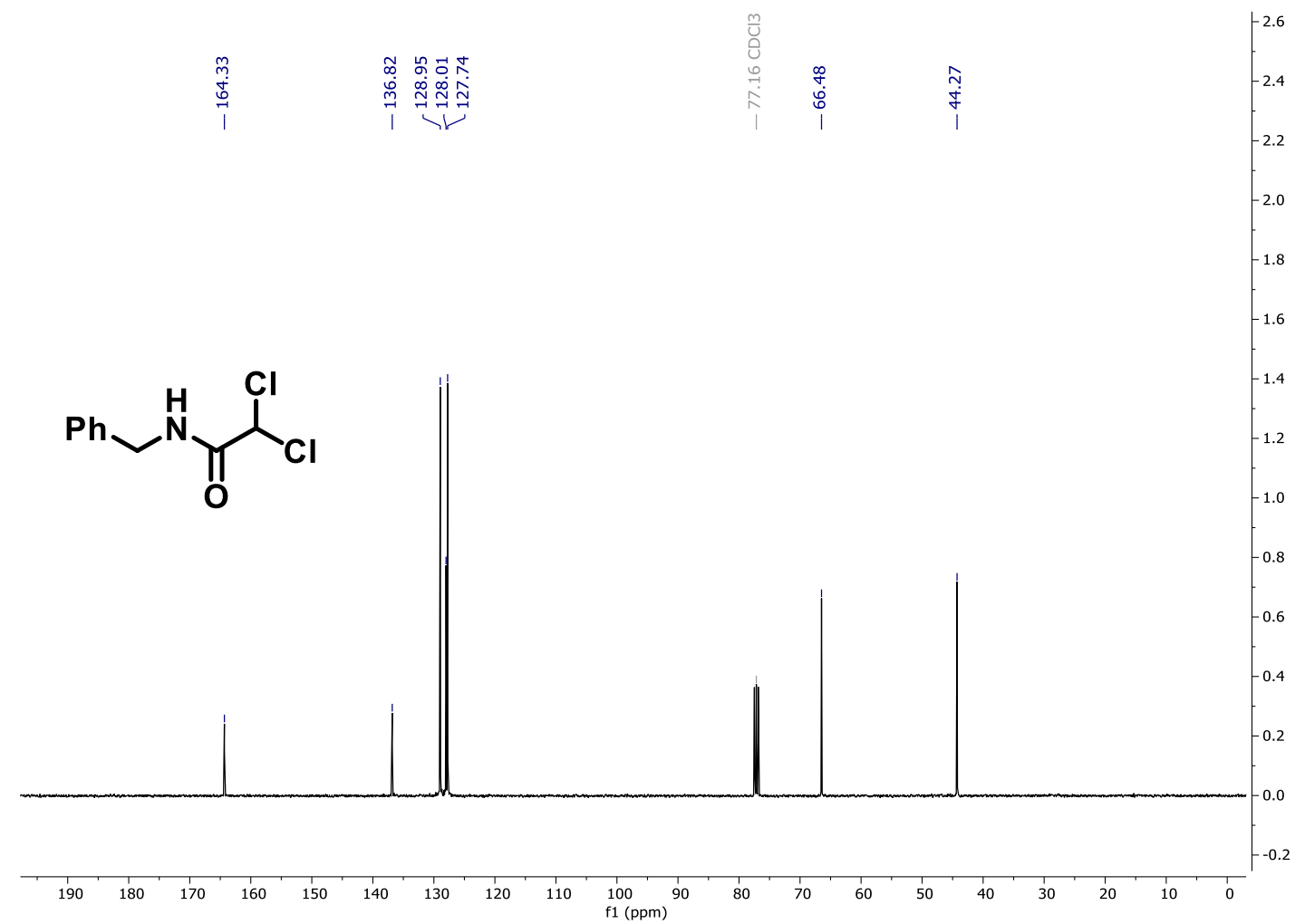


$\mathrm{N}$-(4-Bromobenzyl)-2,2-dichloroacetamide 3v

${ }^{1} \mathrm{H}$ NMR (400 MHz, $\mathrm{CDCl}_{3}$ )

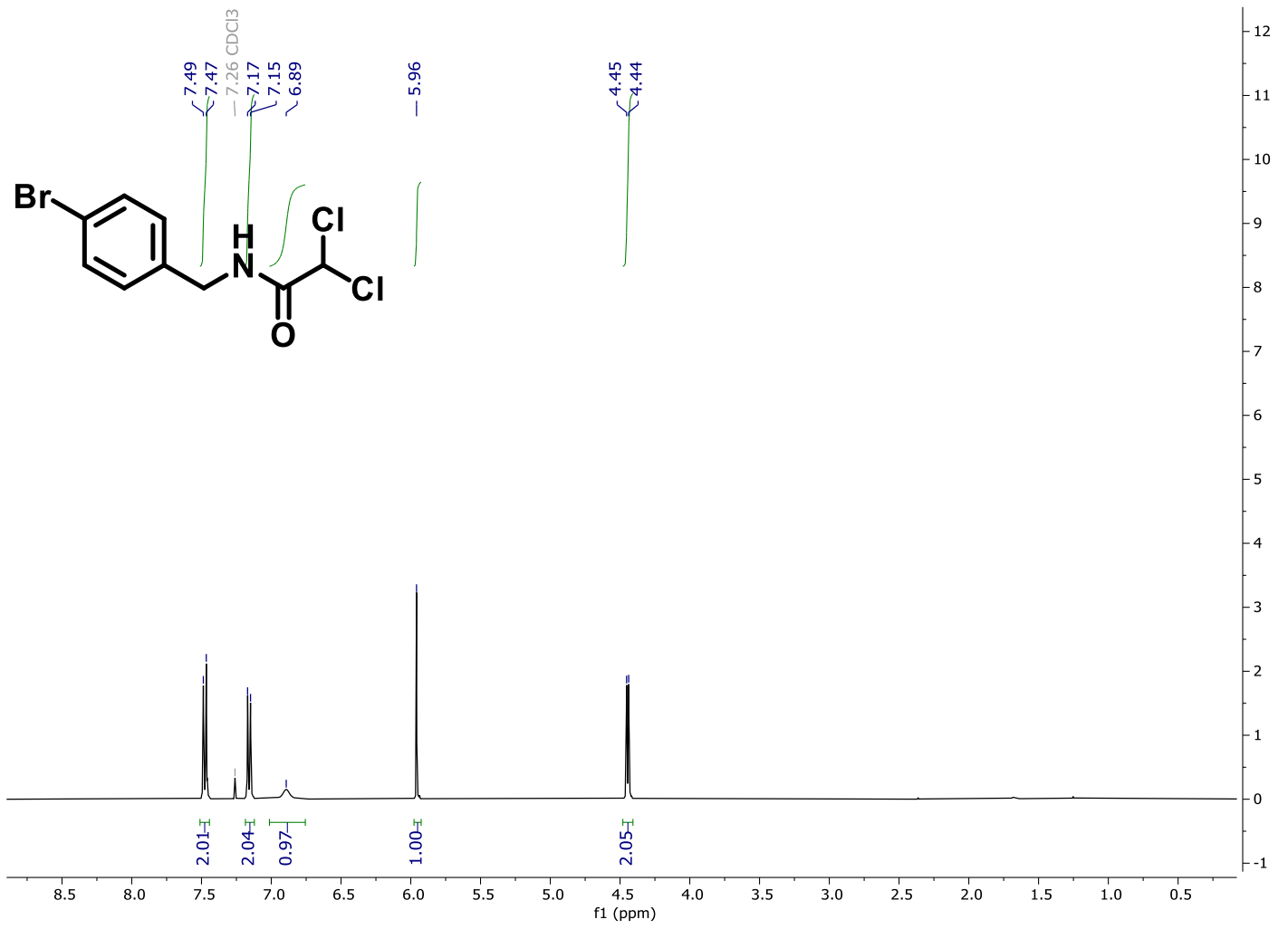


${ }^{13} \mathrm{C}$ NMR (101 MHz, $\mathrm{CDCl}_{3}$ )

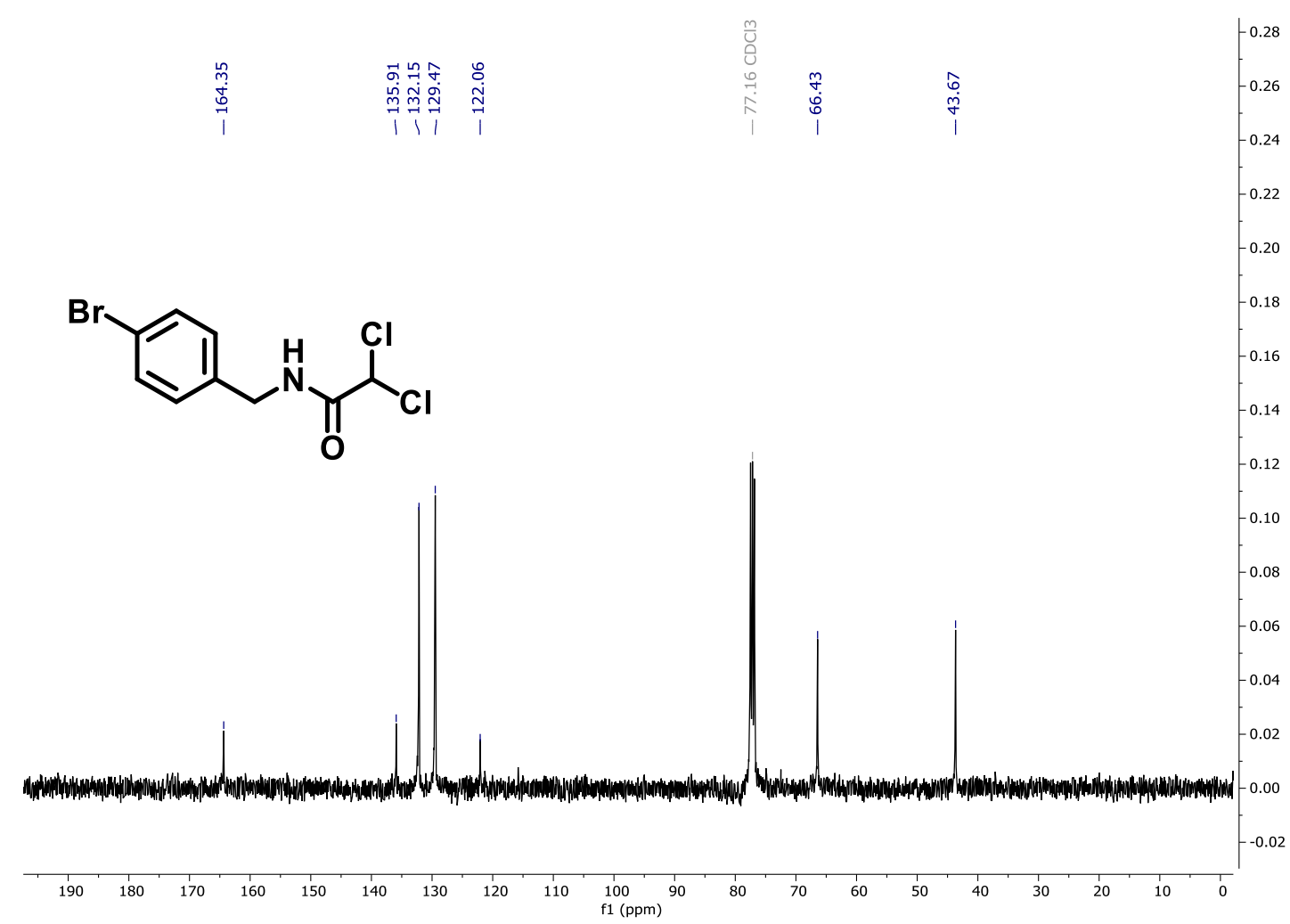


$\mathrm{N}$-(4-Fluorobenzyl)-2,2-dichloroacetamide 3w

${ }^{1} \mathrm{H}$ NMR (400 MHz, $\mathrm{CDCl}_{3}$ )

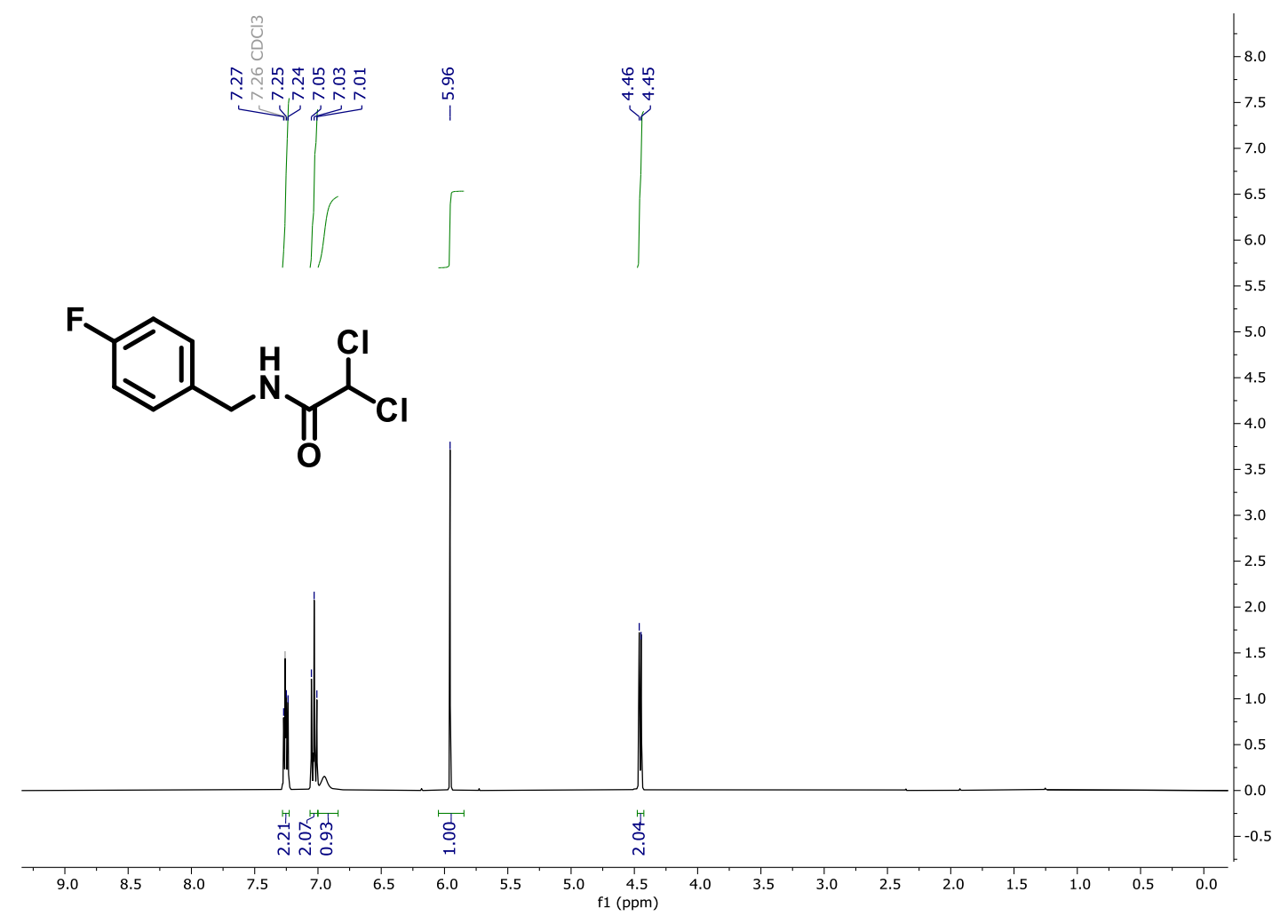


${ }^{13} \mathrm{C}$ NMR (101 MHz, $\left.\mathrm{CDCl}_{3}\right)$

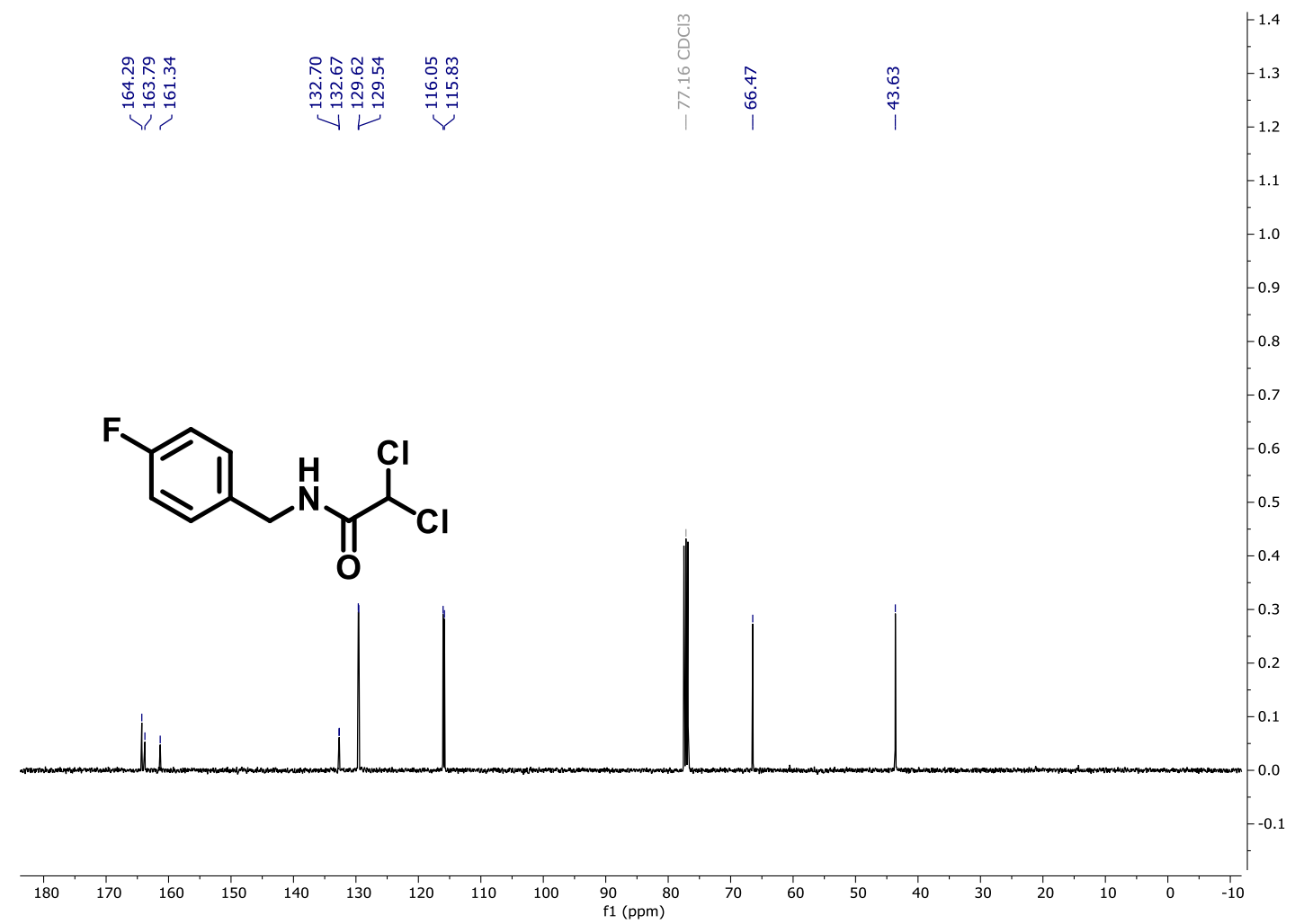


${ }^{19} \mathrm{~F}$ NMR (376 MHz, $\mathrm{CDCl}_{3}$ )

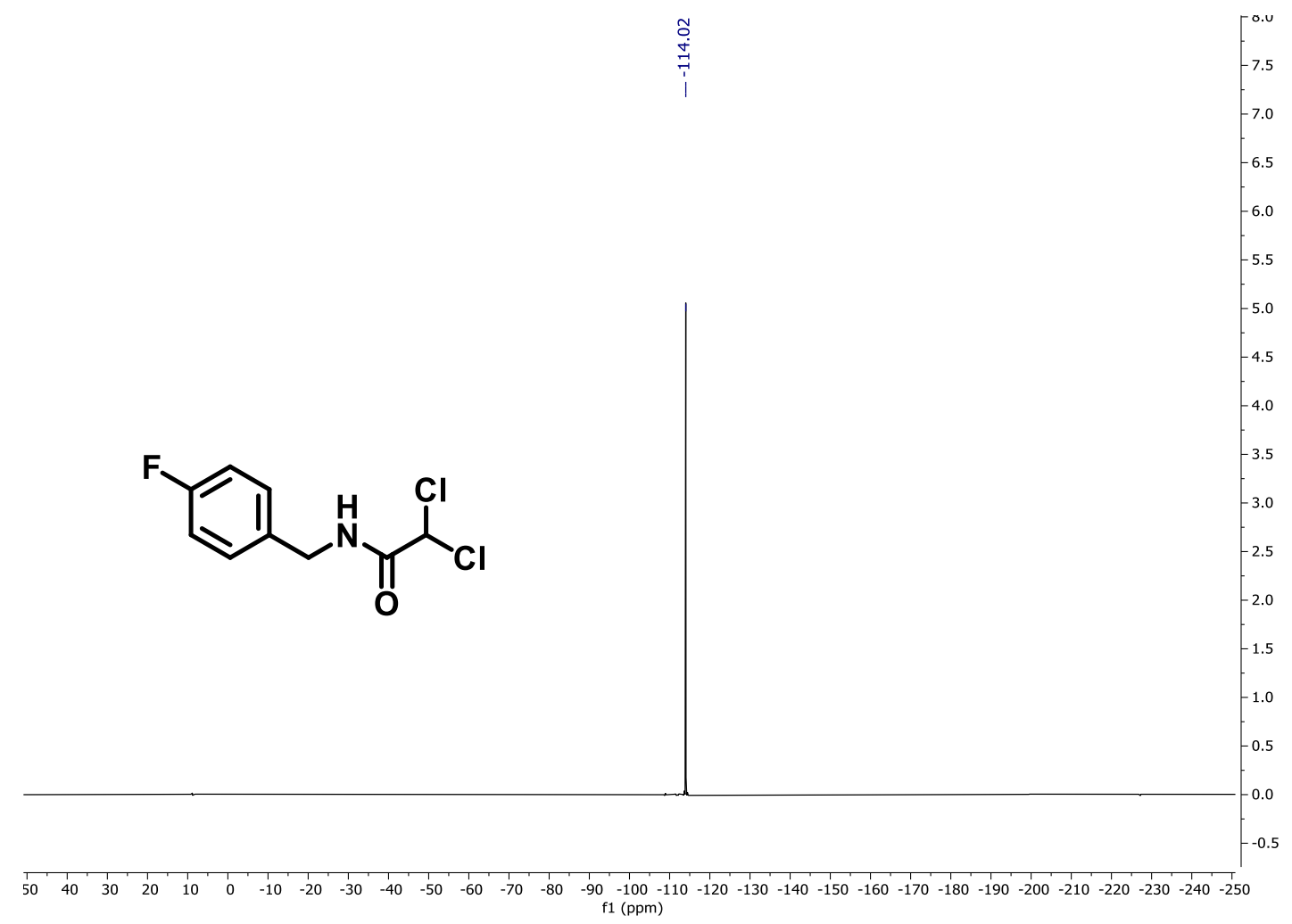


2,2-Dichloro- $N$-(3,5-dimethoxybenzyl)acetamide $3 x$

${ }^{1} \mathrm{H}$ NMR (400 MHz, $\mathrm{CDCl}_{3}$ )

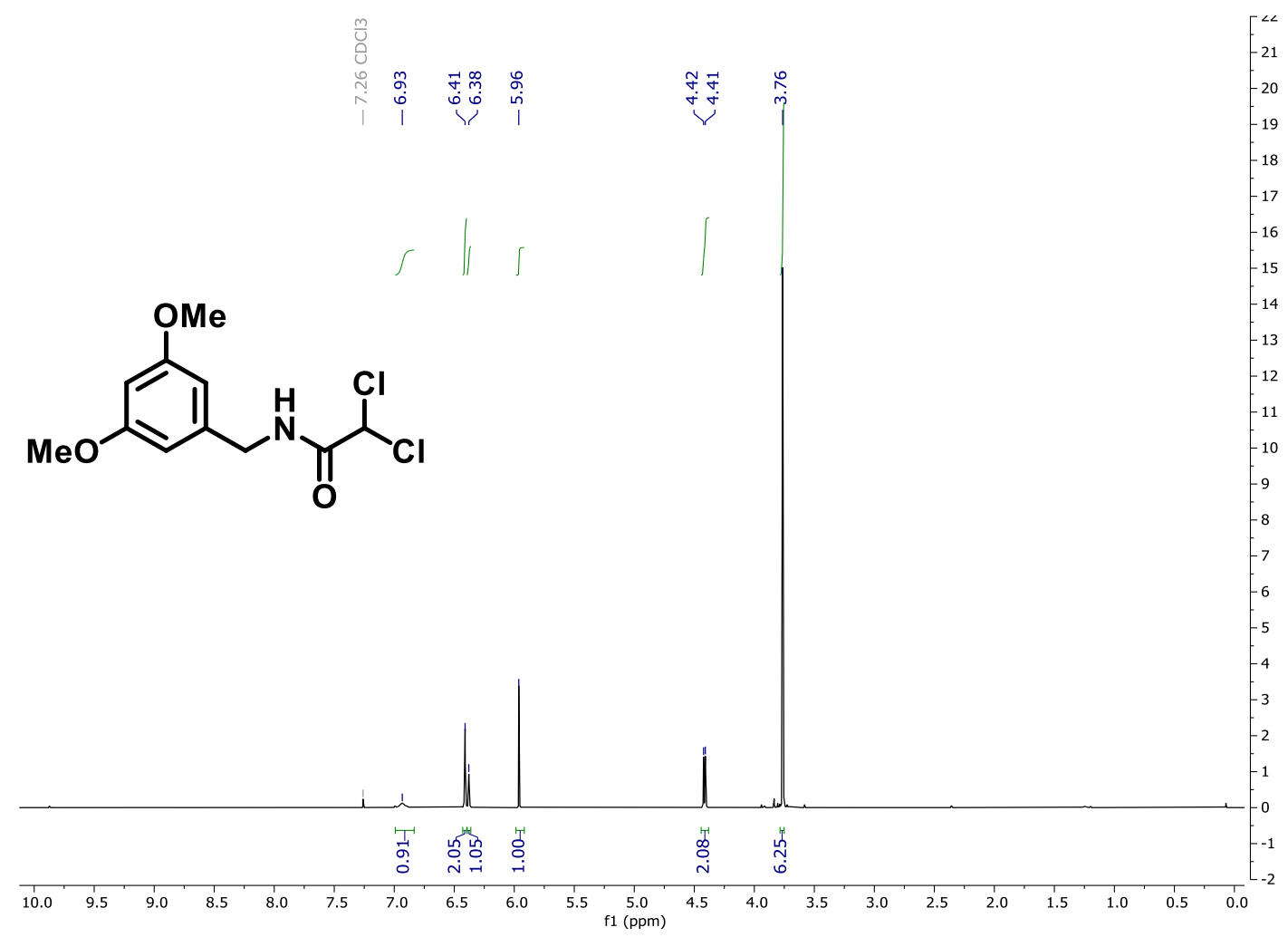


${ }^{13} \mathrm{C}$ NMR (101 MHz, $\left.\mathrm{CDCl}_{3}\right)$

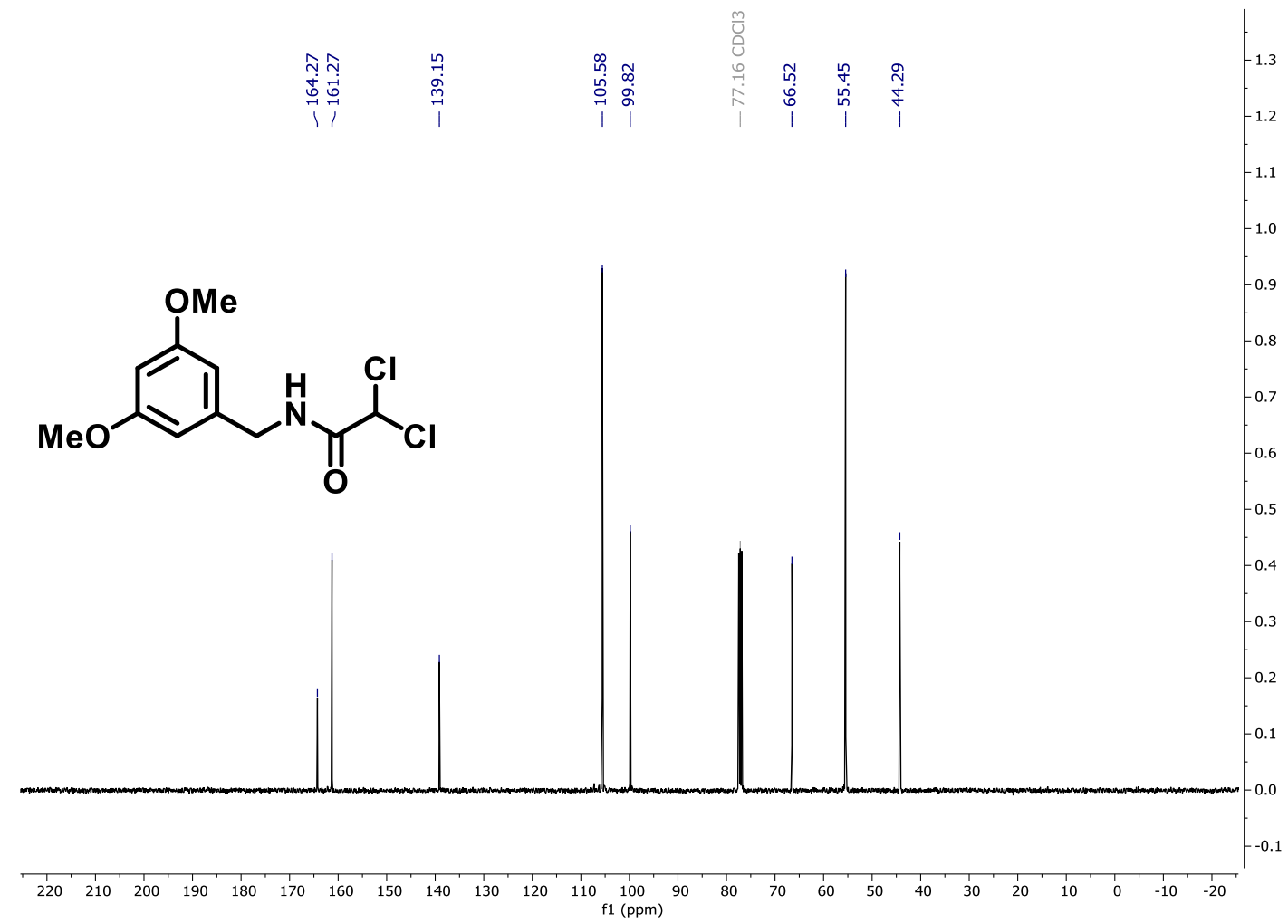


2,2-Dichloro- $N$-(naphthalen-1-ylmethyl)acetamide 3y

${ }^{1} \mathrm{H}$ NMR (400 MHz, $\mathrm{CDCl}_{3}$ )

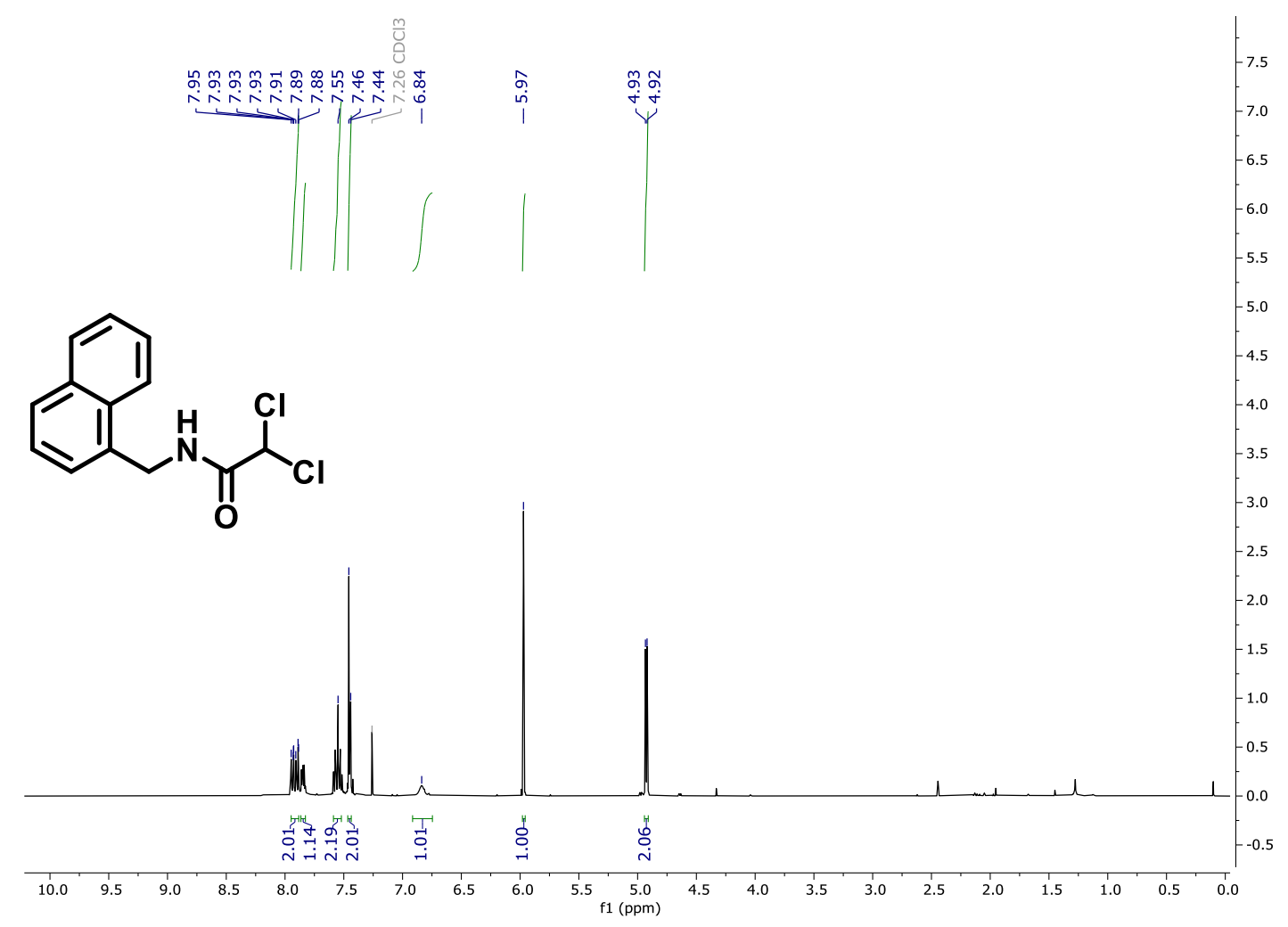


${ }^{13} \mathrm{C}$ NMR (101 MHz, $\left.\mathrm{CDCl}_{3}\right)$

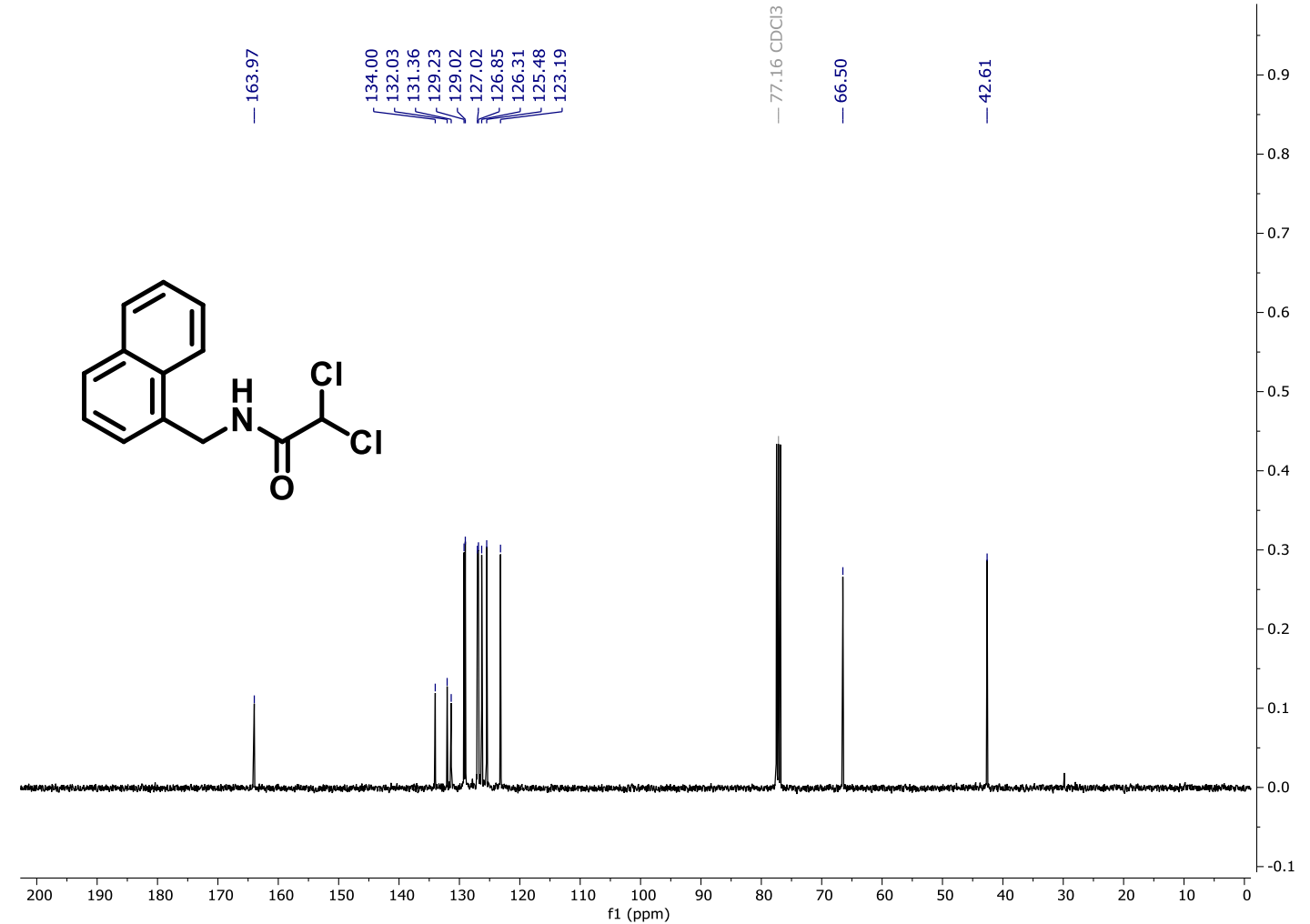


2,2-Dichloro- $N$-cyclobutylacetamide $3 z$

${ }^{1} \mathrm{H}$ NMR (400 MHz, $\mathrm{CDCl}_{3}$ )

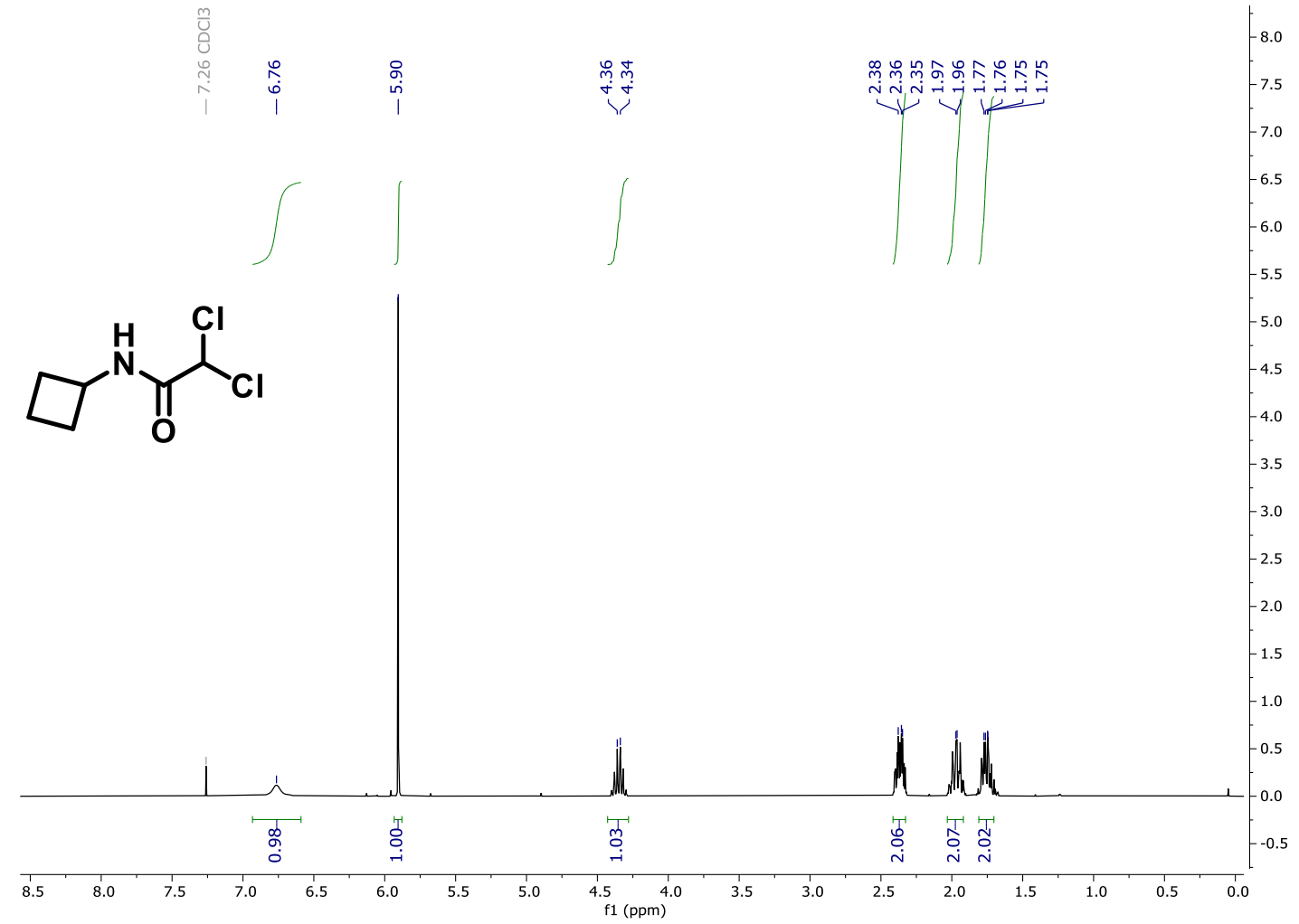


${ }^{13} \mathrm{C}$ NMR (101 MHz, $\left.\mathrm{CDCl}_{3}\right)$

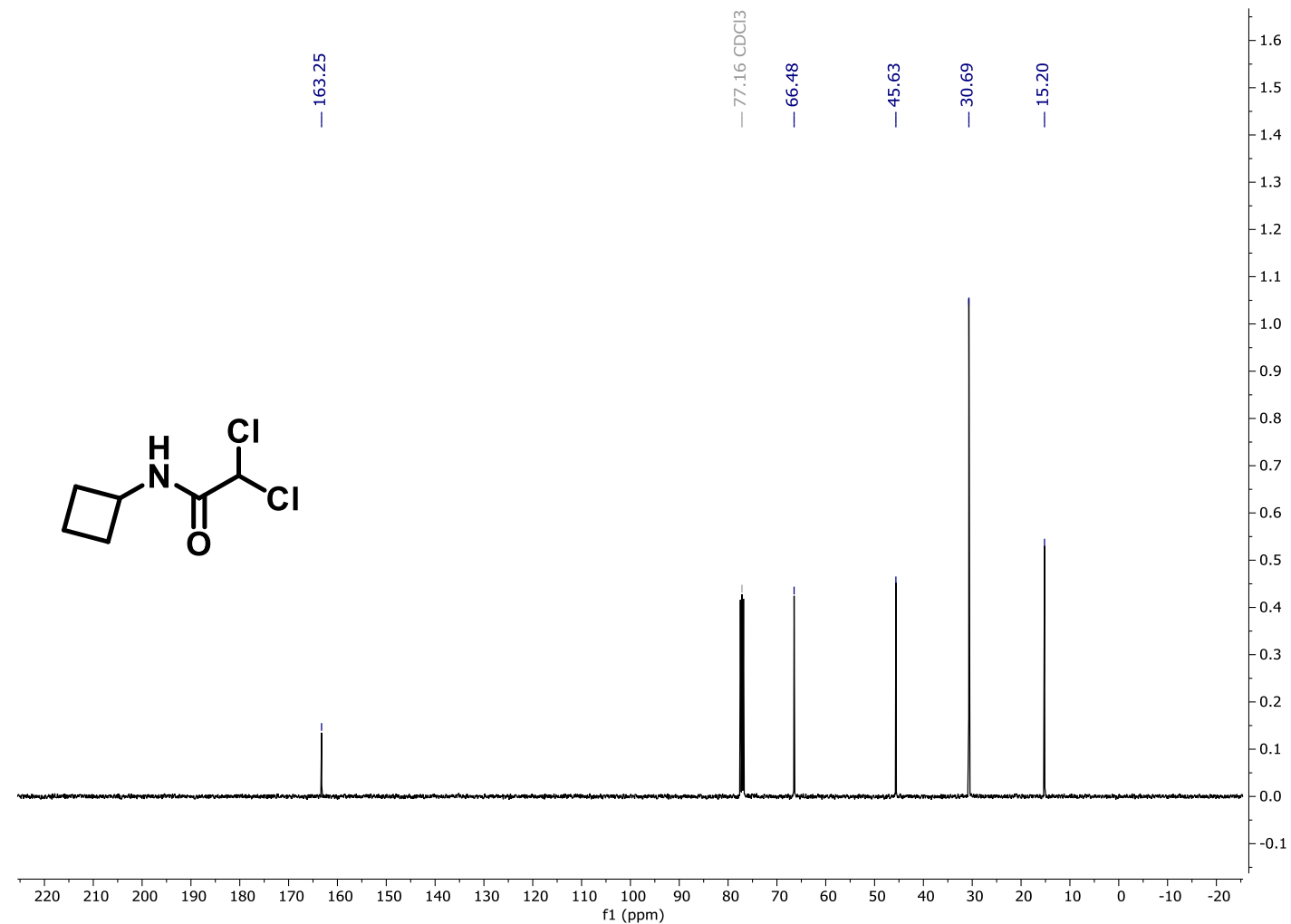


2,2-Dichloro- $N$-cyclopentylacetamide 3aa

${ }^{1} \mathrm{H}$ NMR (400 MHz, $\mathrm{CDCl}_{3}$ )

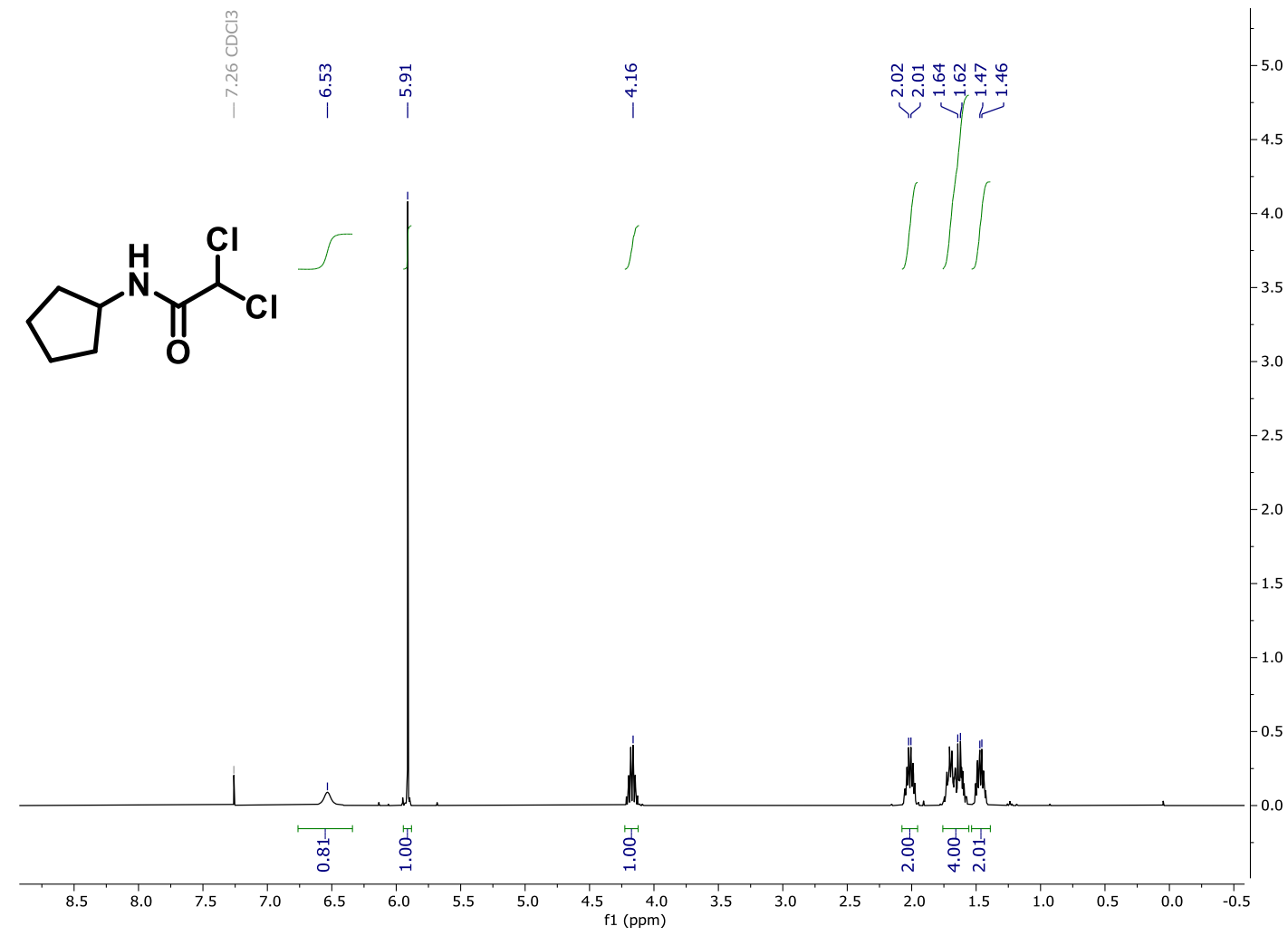


${ }^{13} \mathrm{C}$ NMR (101 MHz, $\left.\mathrm{CDCl}_{3}\right)$

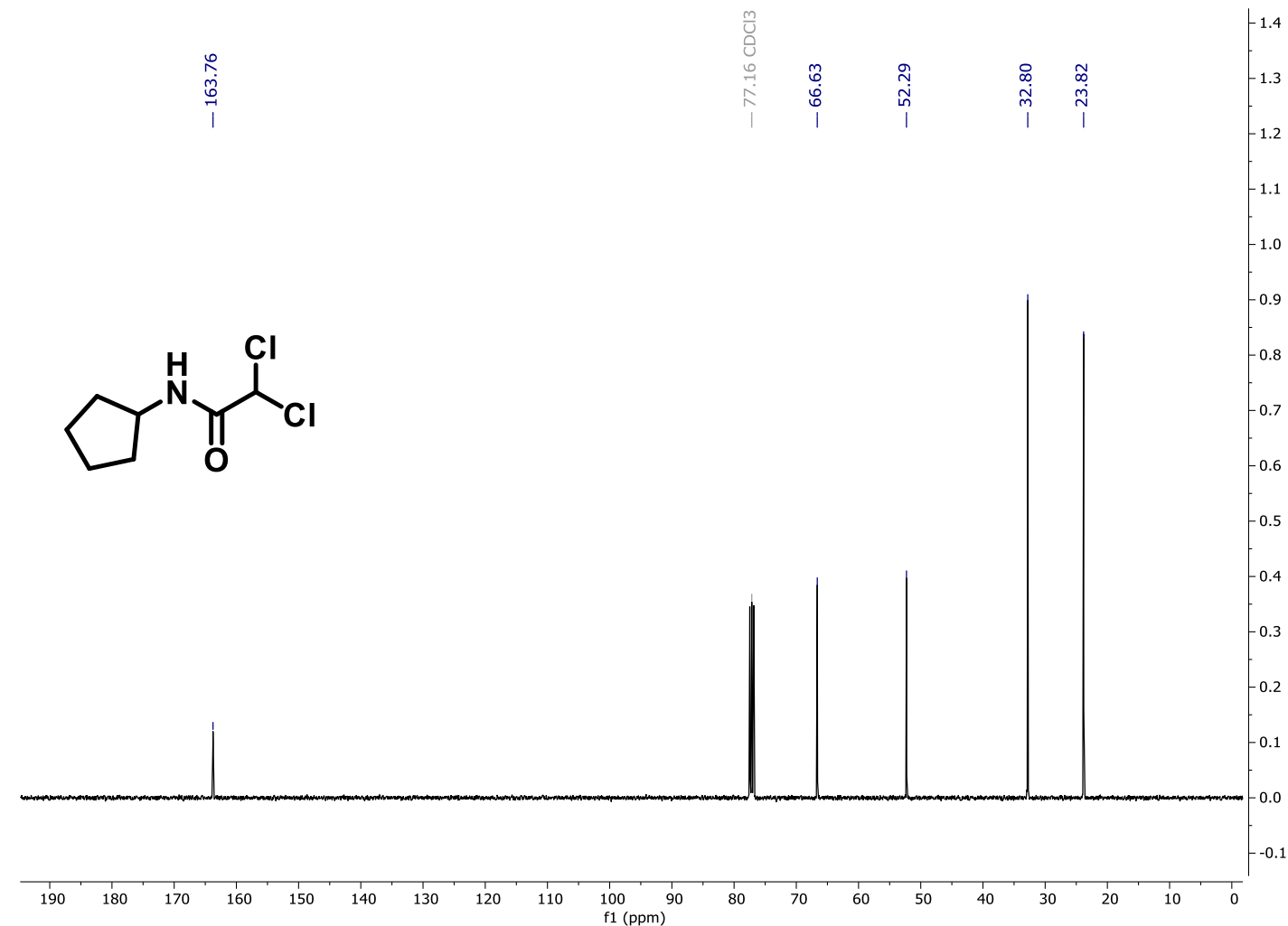


2,2-Dichloro- $N$-cyclohexylacetamide 3ab

${ }^{1} \mathrm{H}$ NMR (400 MHz, $\mathrm{CDCl}_{3}$ )

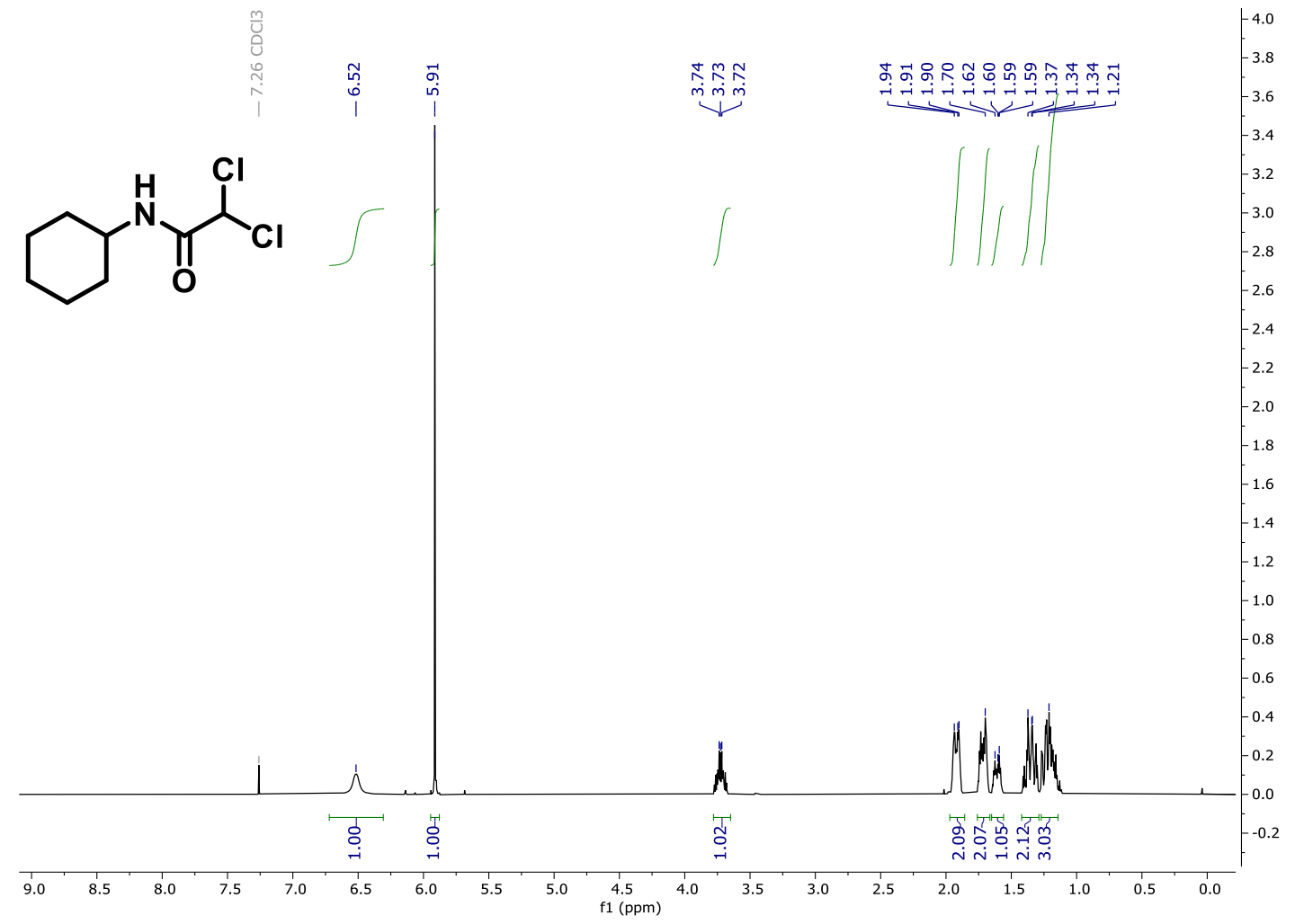


${ }^{13} \mathrm{C}$ NMR (101 MHz, $\mathrm{CDCl}_{3}$ )<smiles>O=C(NC1CCCCC1)C(Cl)Cl</smiles>

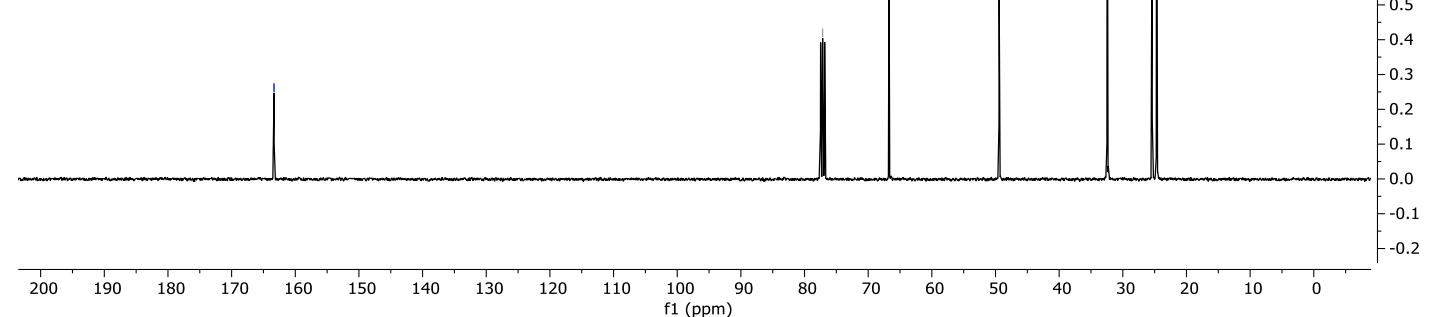


2,2-Dichloro-N-cycloheptylacetamide 3ac

${ }^{1} \mathrm{H}$ NMR (400 MHz, $\mathrm{CDCl}_{3}$ )

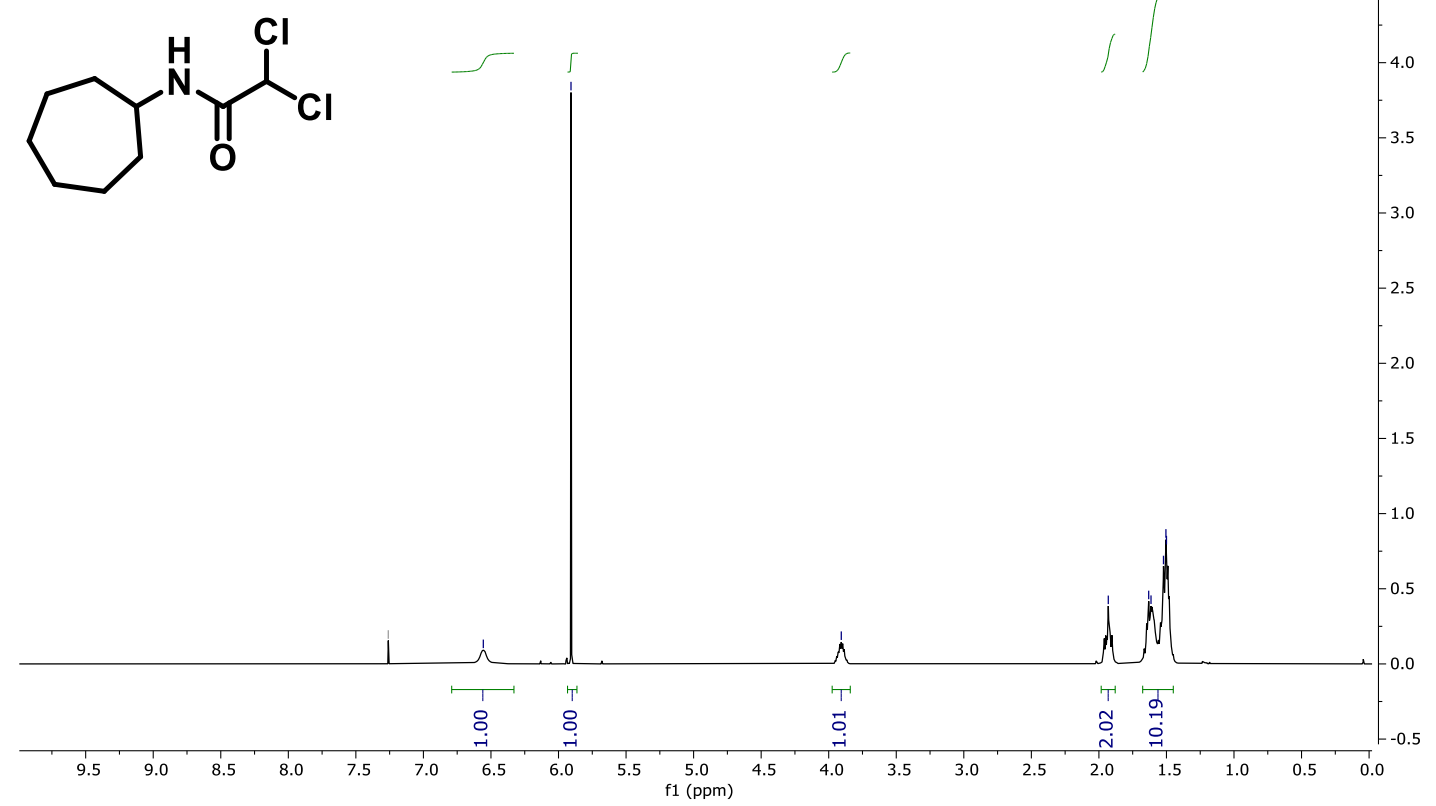


${ }^{13} \mathrm{C}$ NMR (101 MHz, $\left.\mathrm{CDCl}_{3}\right)$

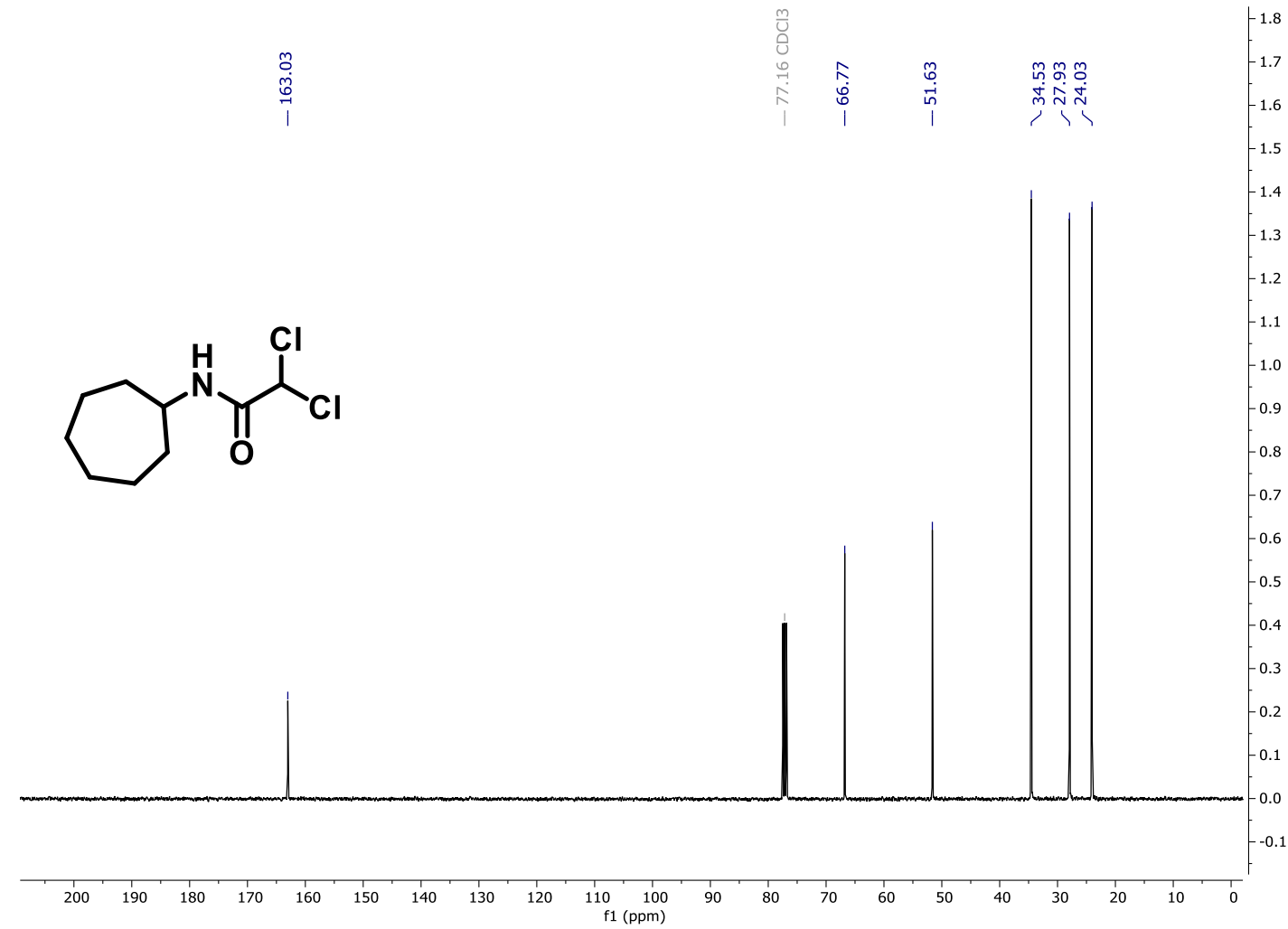


2,2-Dichloro- $N$-(pentan-3-yl)acetamide 3ad

${ }^{1} \mathrm{H} \mathrm{NMR}\left(400 \mathrm{MHz}, \mathrm{CDCl}_{3}\right)$

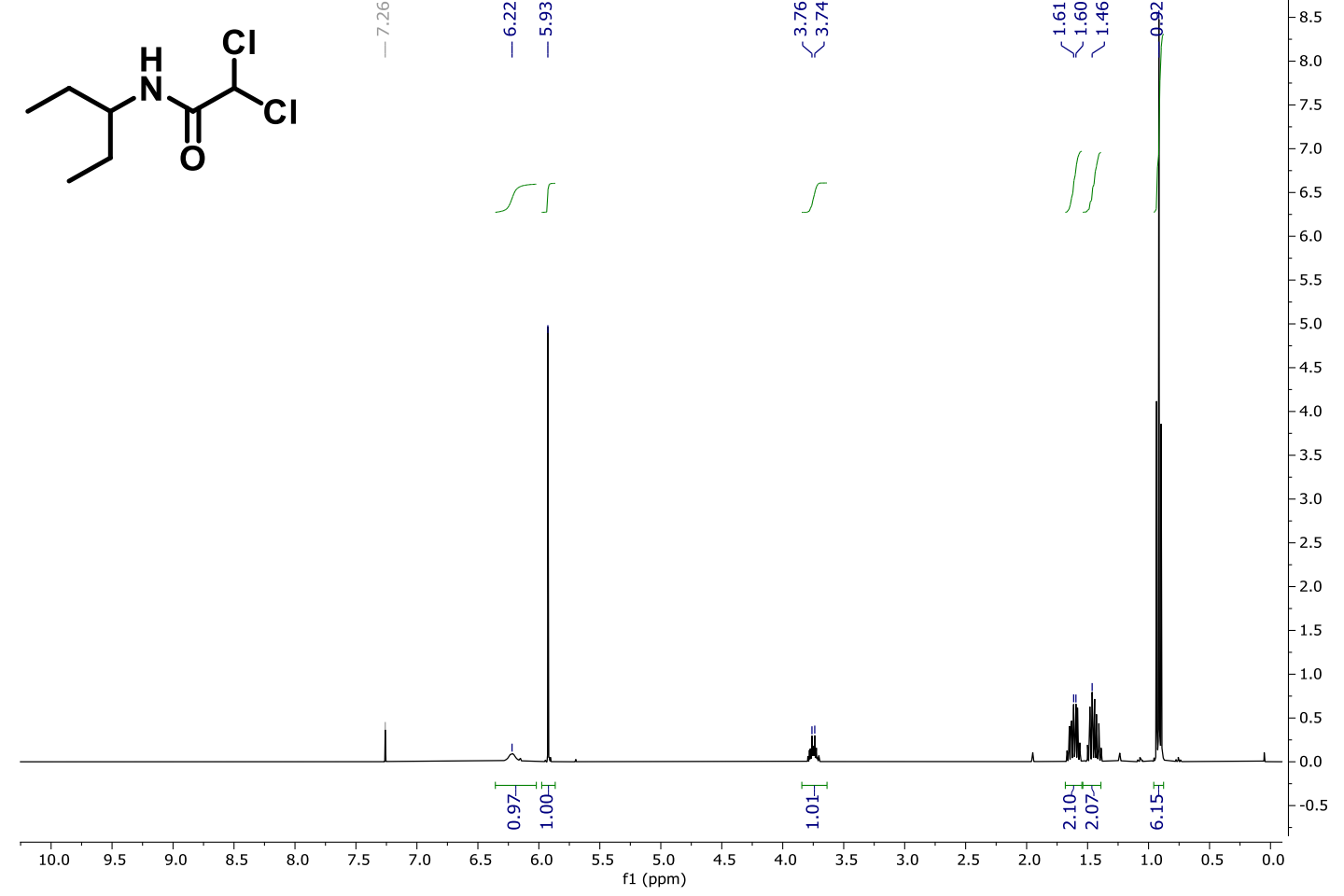


${ }^{13} \mathrm{C}$ NMR (101 MHz, $\left.\mathrm{CDCl}_{3}\right)$

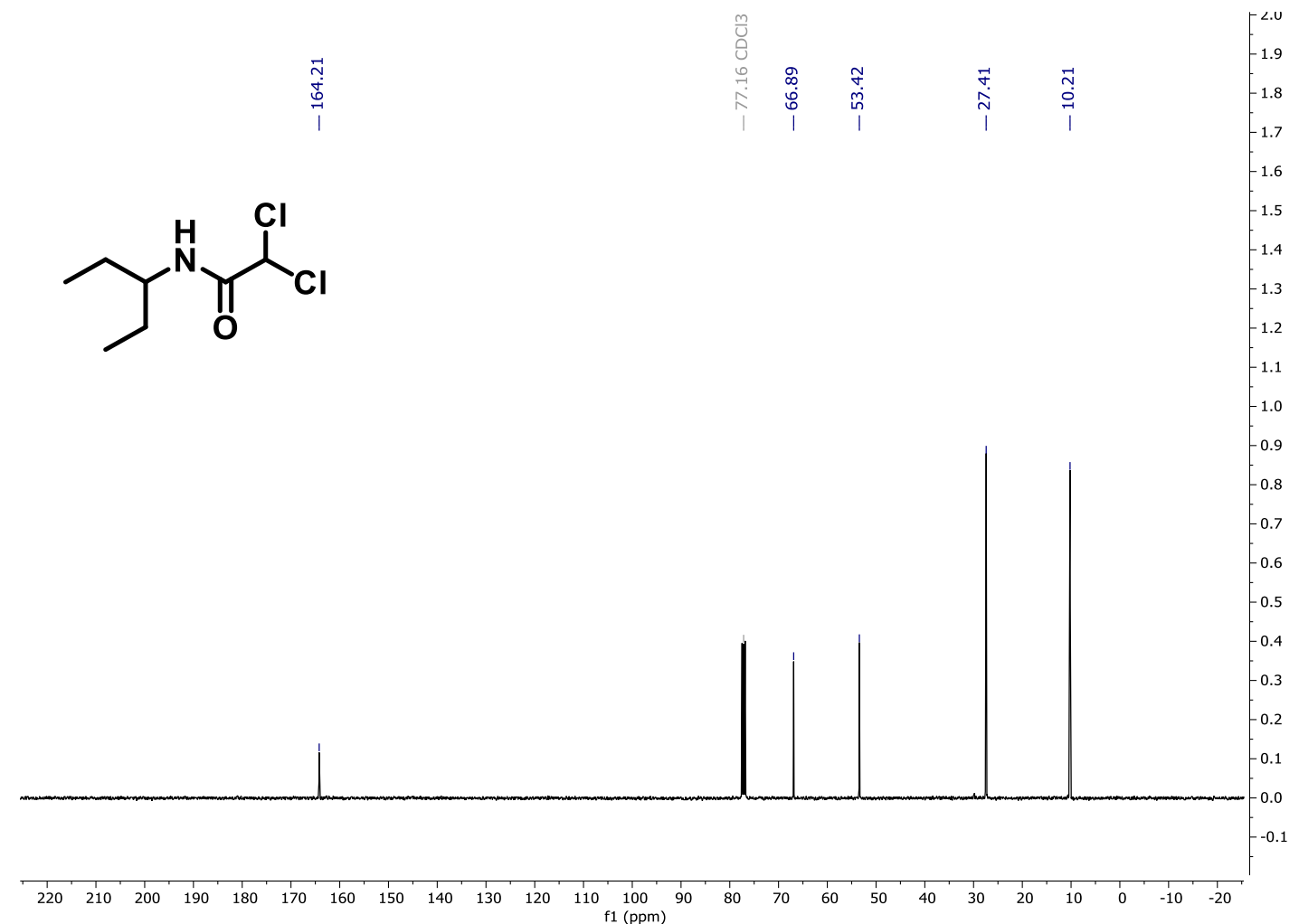


2,2-Dichloro- $N$-butylacetamide 3ae

${ }^{1} \mathrm{H}$ NMR (400 MHz, $\mathrm{CDCl}_{3}$ )

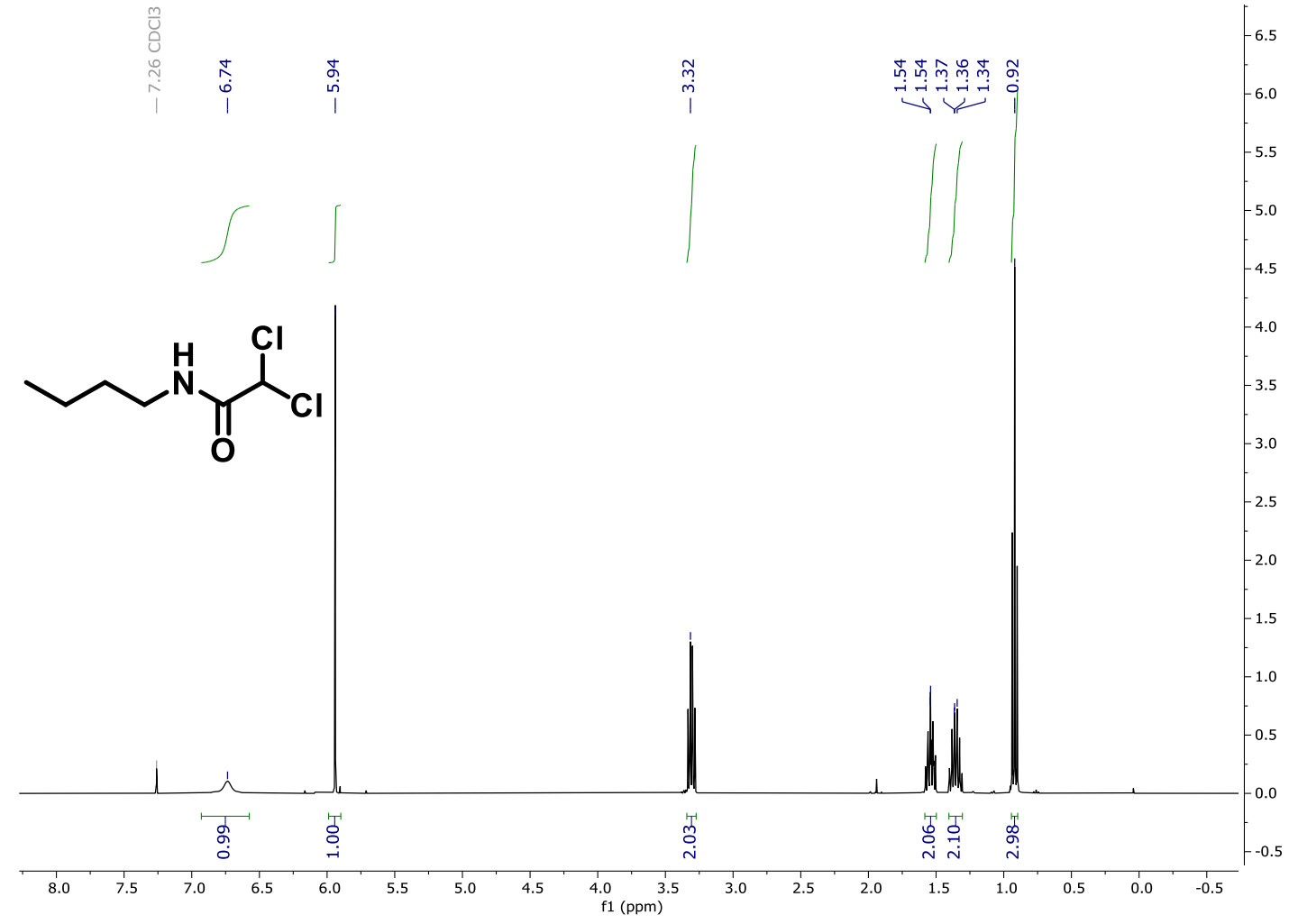


${ }^{13} \mathrm{C}$ NMR (101 MHz, $\left.\mathrm{CDCl}_{3}\right)$

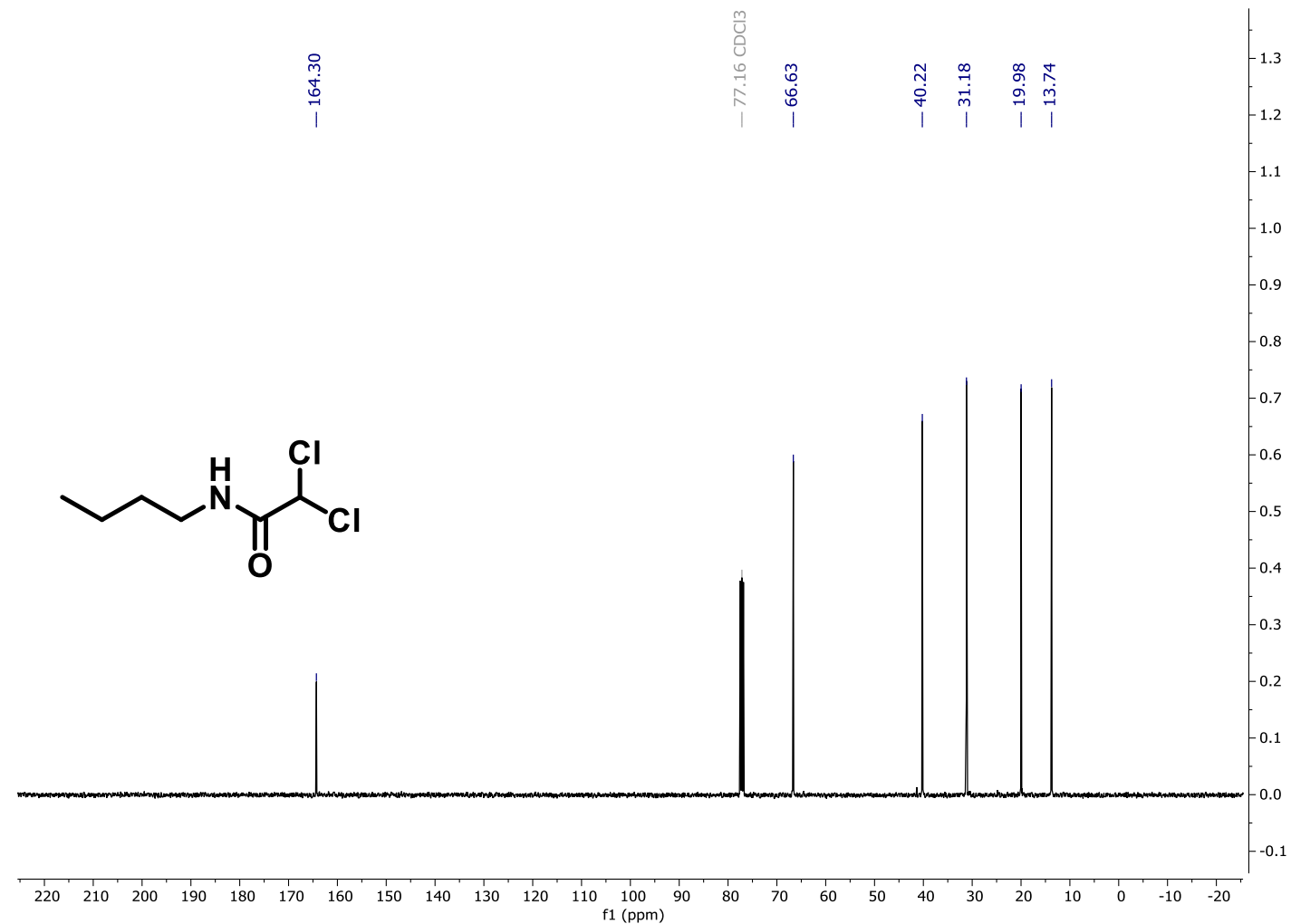


2,2-Dichloro- $N$-octylacetamide 3af

${ }^{1} \mathrm{H}$ NMR (400 MHz, $\mathrm{CDCl}_{3}$ )

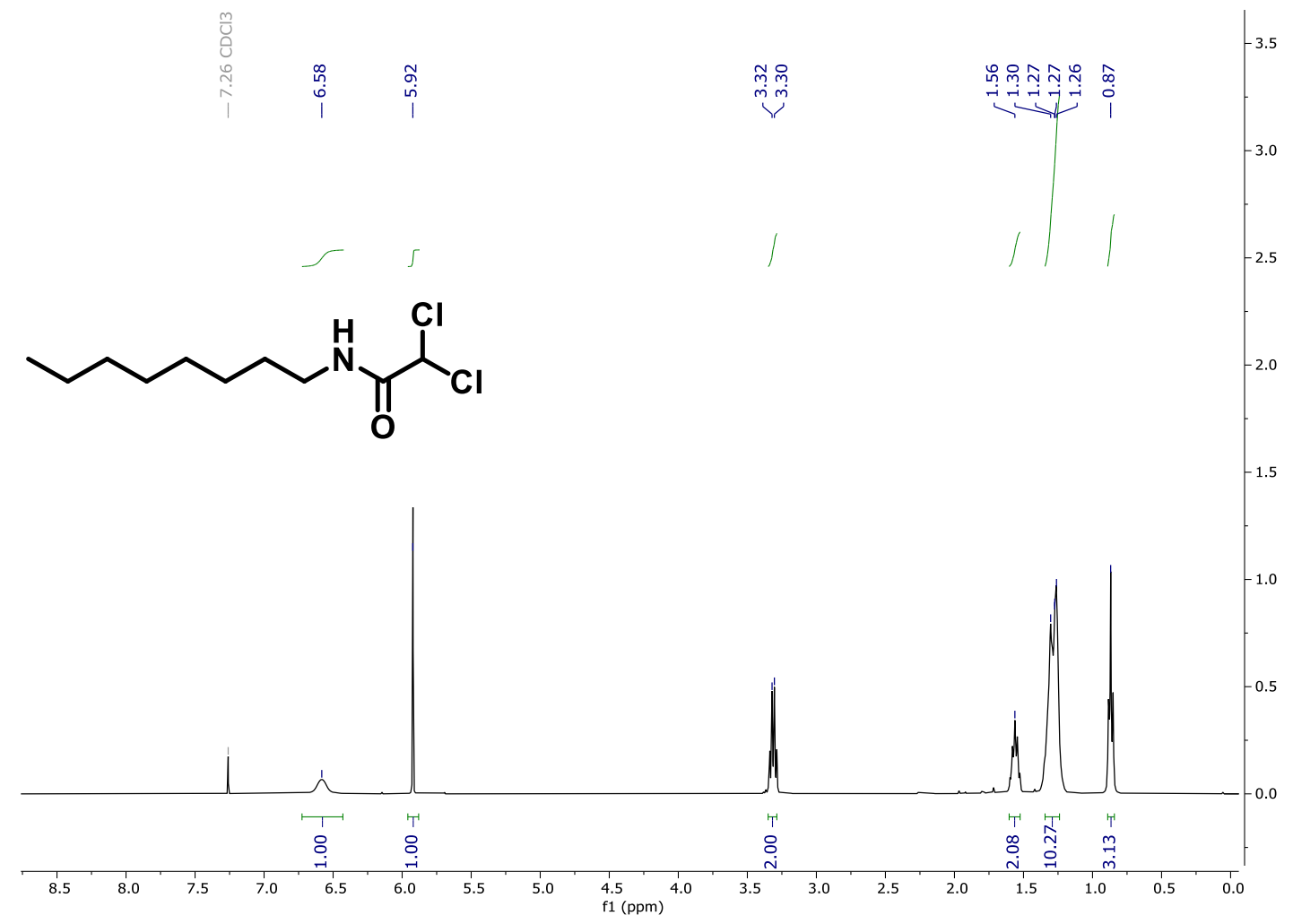


${ }^{13} \mathrm{C}$ NMR (101 MHz, $\left.\mathrm{CDCl}_{3}\right)$

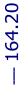

$\mathscr{0}$
$\dot{0}$
$\dot{0}$
1

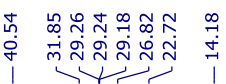

$\sim_{\prod_{0}^{N}}^{C l}$

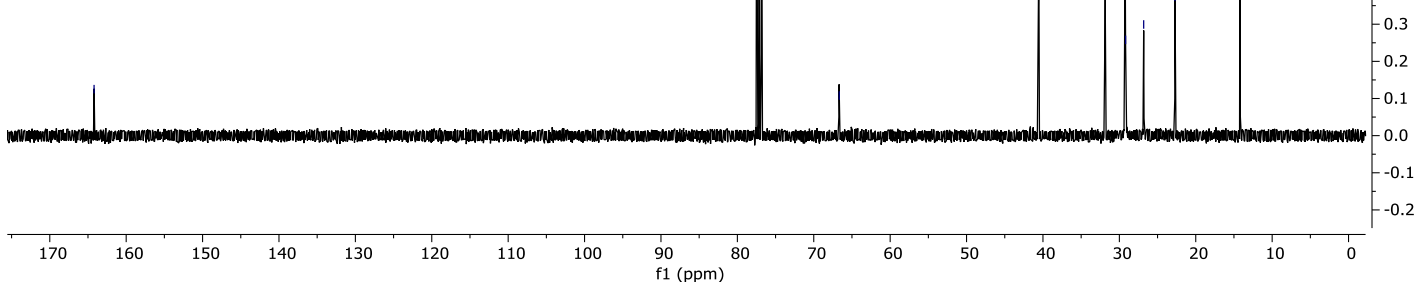


2,2-Dichloro- $N$-(2-phenylethyl)acetamide 3ag

${ }^{1} \mathrm{H}$ NMR (400 MHz, $\mathrm{CDCl}_{3}$ )

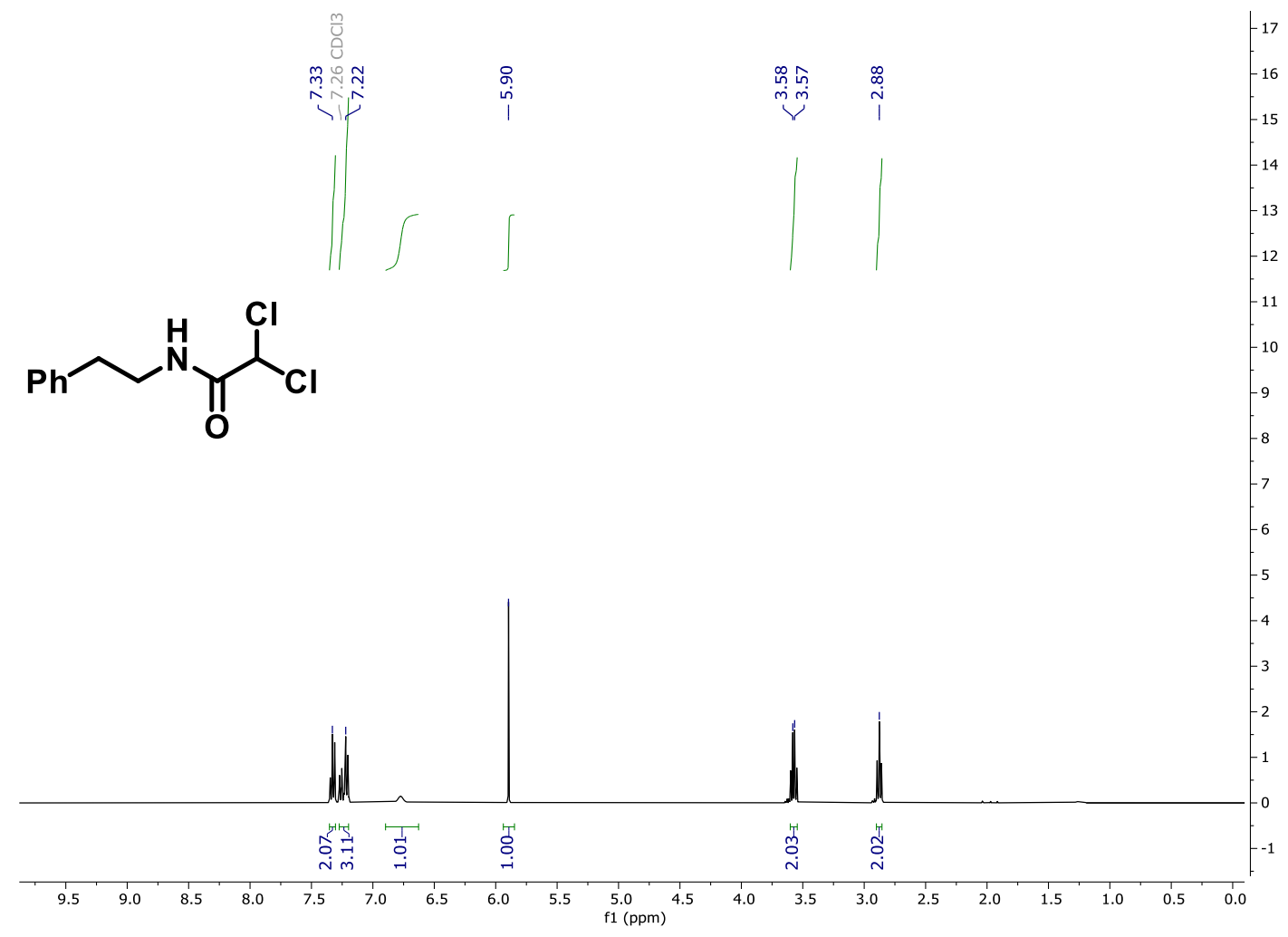


${ }^{13} \mathrm{C}$ NMR (101 MHz, $\mathrm{CDCl}_{3}$ )

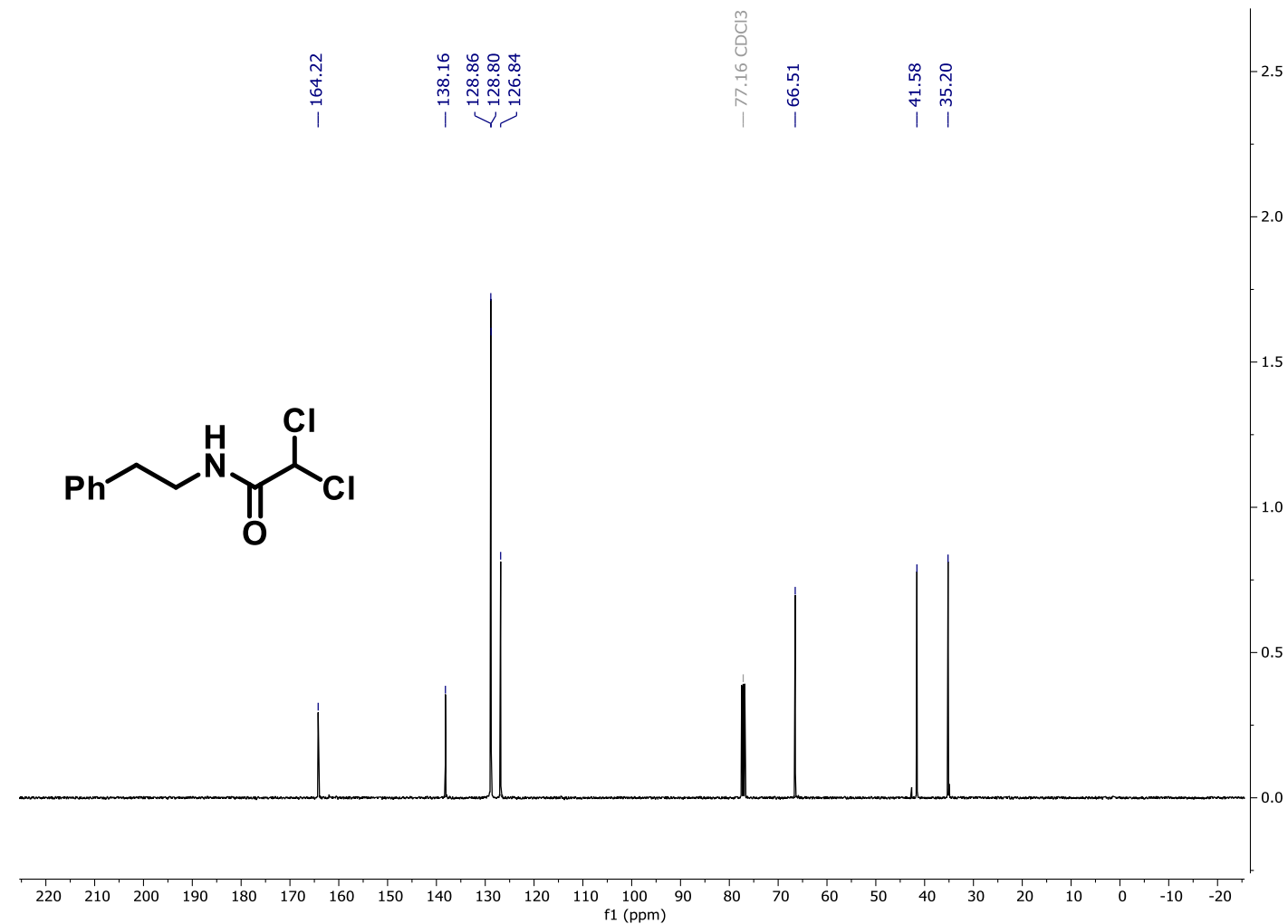


2,2-Dichloro- $N$-(3-phenylpropyl)acetamide 3ah

${ }^{1} \mathrm{H}$ NMR $\left(400 \mathrm{MHz}, \mathrm{CDCl}_{3}\right)$

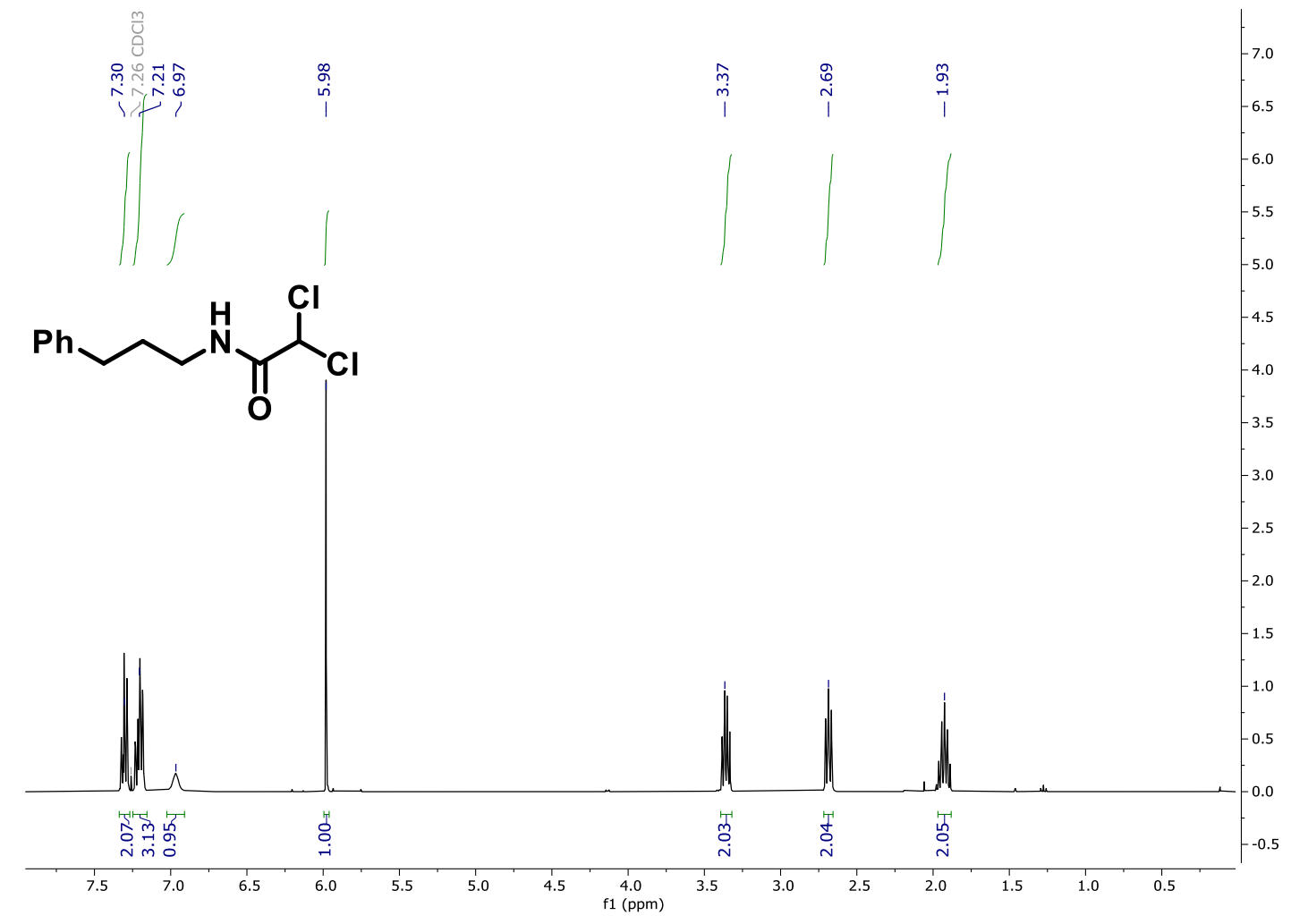

S115 
${ }^{13} \mathrm{C}$ NMR (101 MHz, $\mathrm{CDCl}_{3}$ )

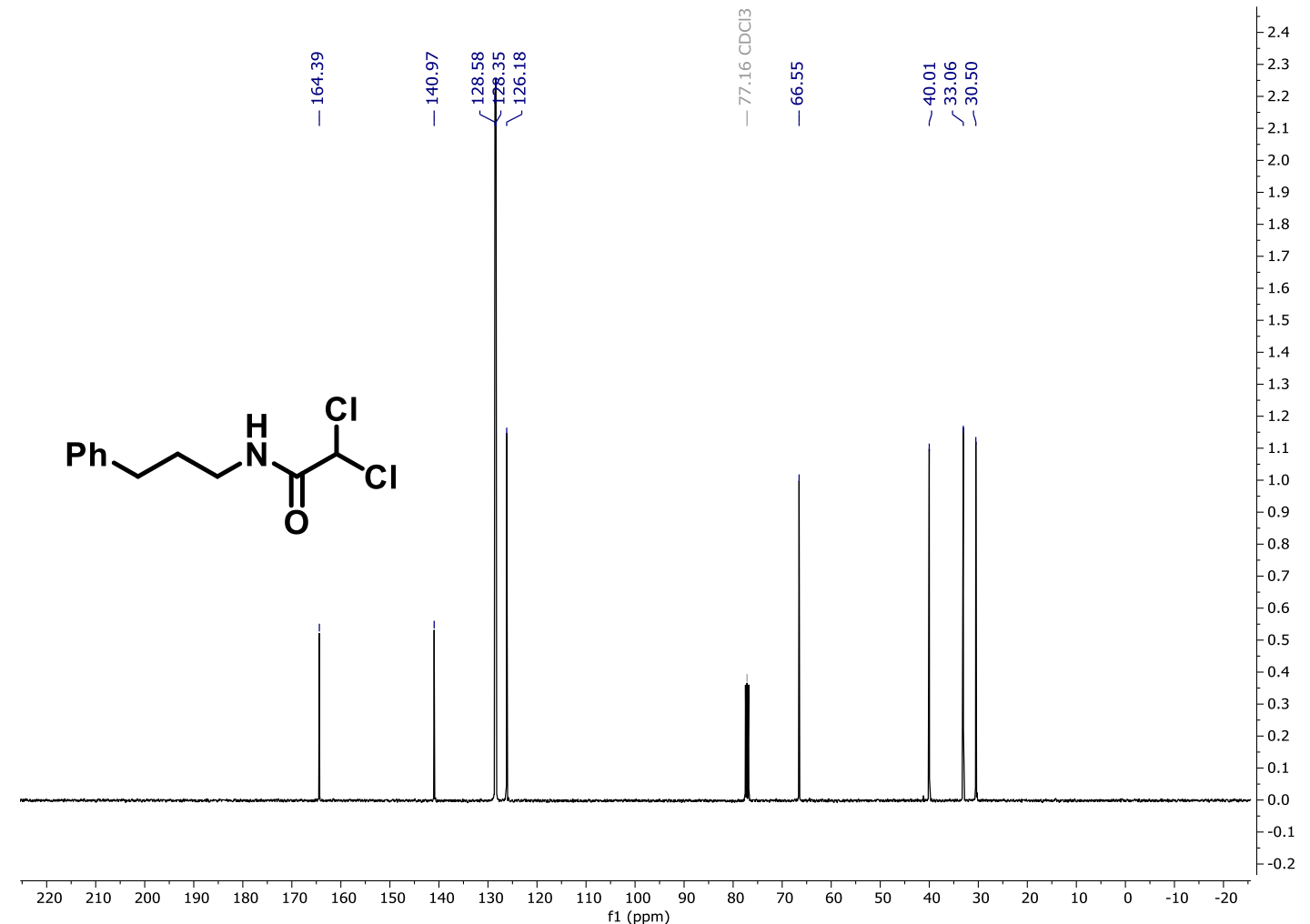


Ethyl (2,2-dichloroacetyl)phenylalaninate 3ai

${ }^{1} \mathrm{H}$ NMR (400 MHz, $\mathrm{CDCl}_{3}$ )

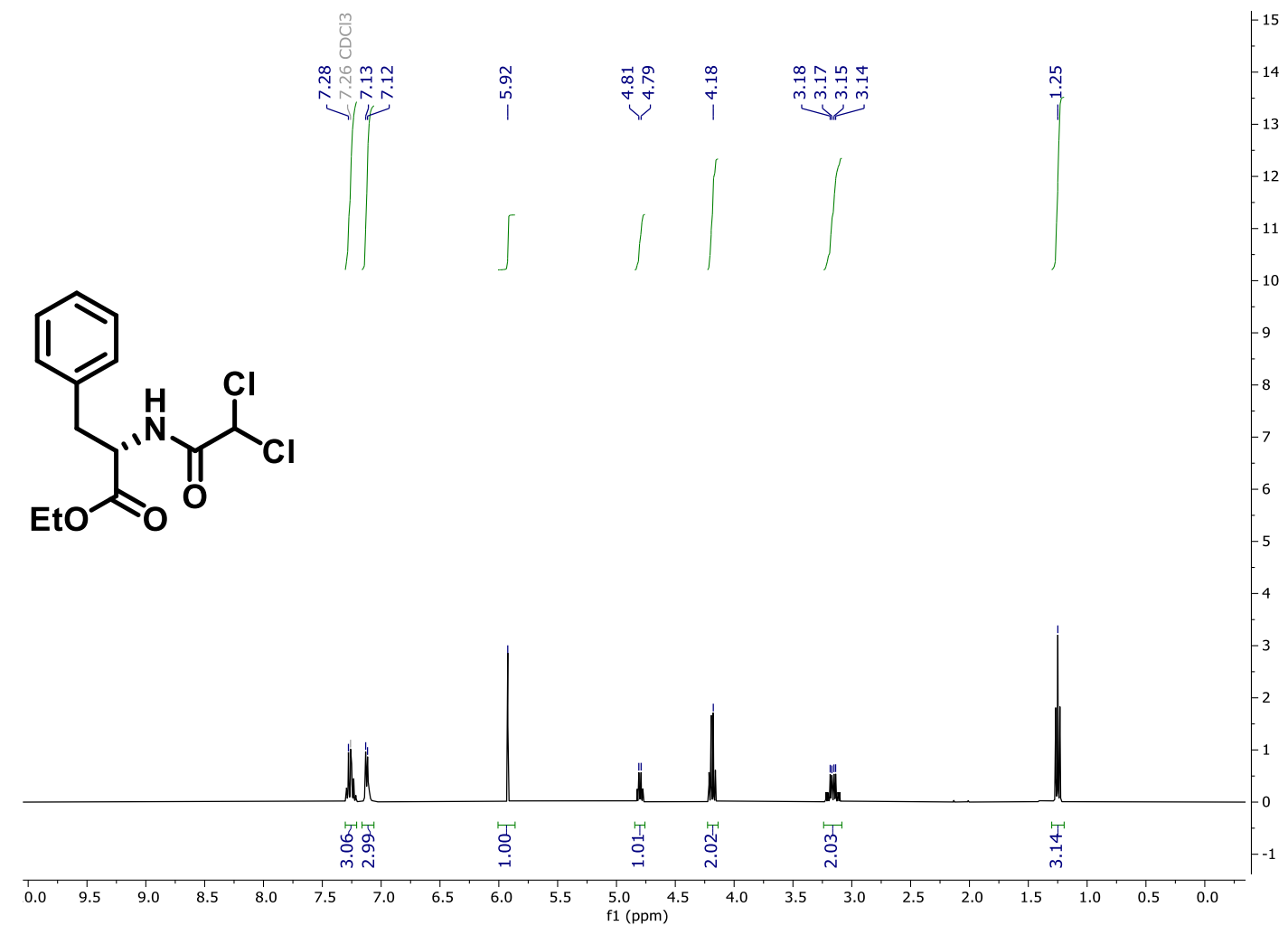


${ }^{13} \mathrm{C}$ NMR (101 MHz, $\left.\mathrm{CDCl}_{3}\right)$

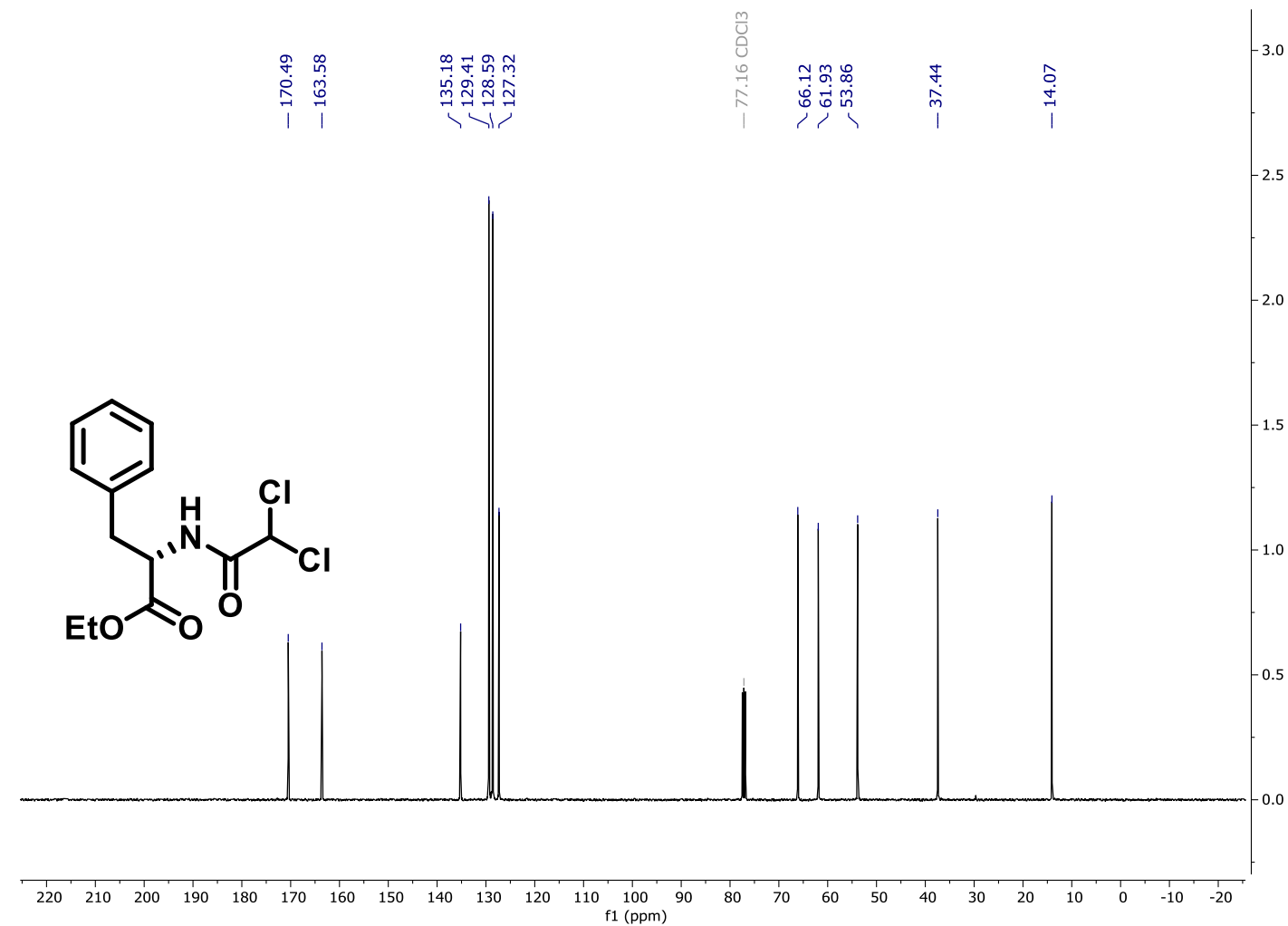


Methyl (S)-3-(4-(tert-butoxy)phenyl)-2-(2,2-dichloroacetamido)propanoate 3aj ${ }^{1} \mathrm{H}$ NMR $\left(400 \mathrm{MHz}, \mathrm{CDCl}_{3}\right)$

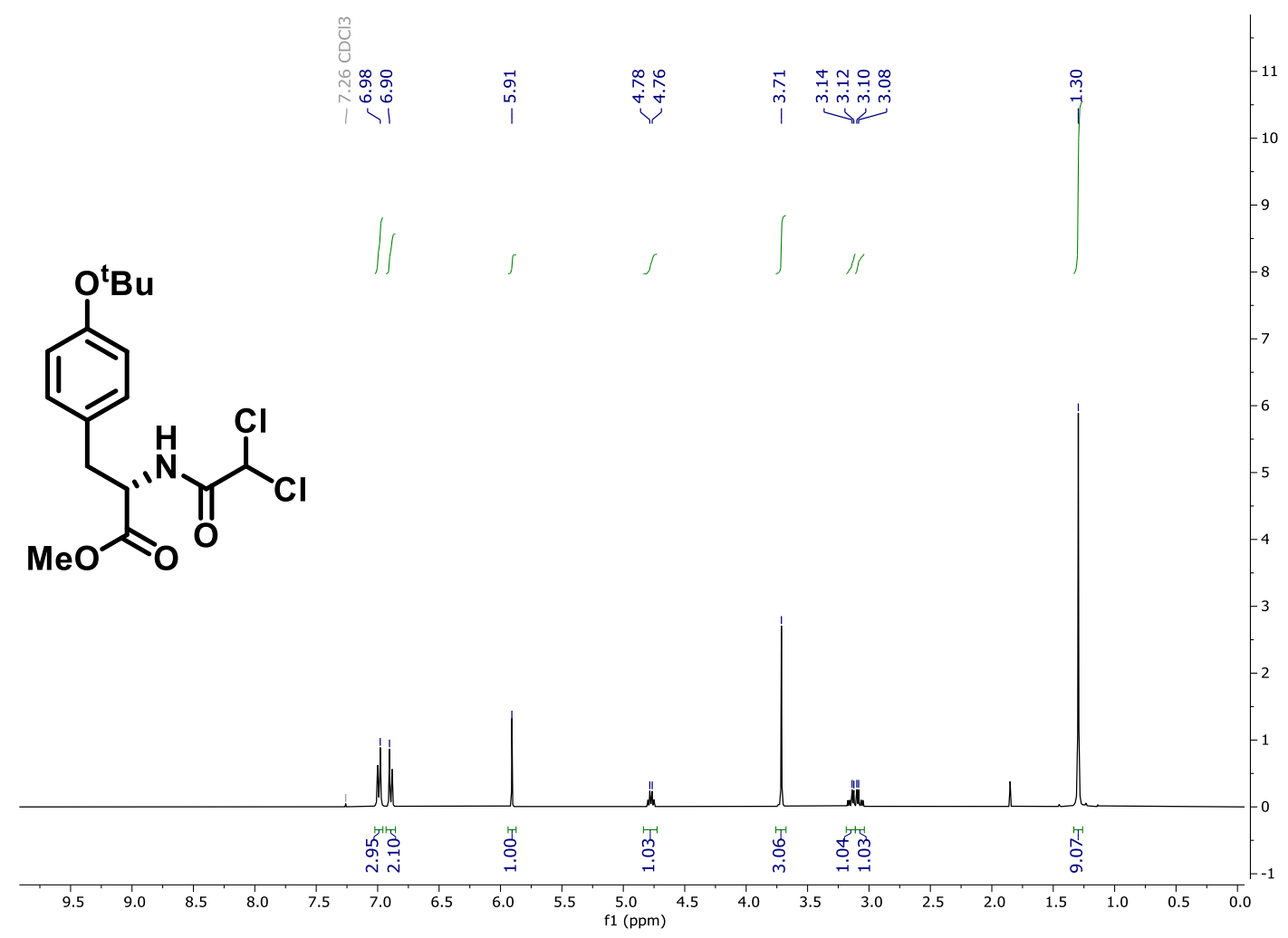


${ }^{13} \mathrm{C}$ NMR (101 MHz, $\left.\mathrm{CDCl}_{3}\right)$

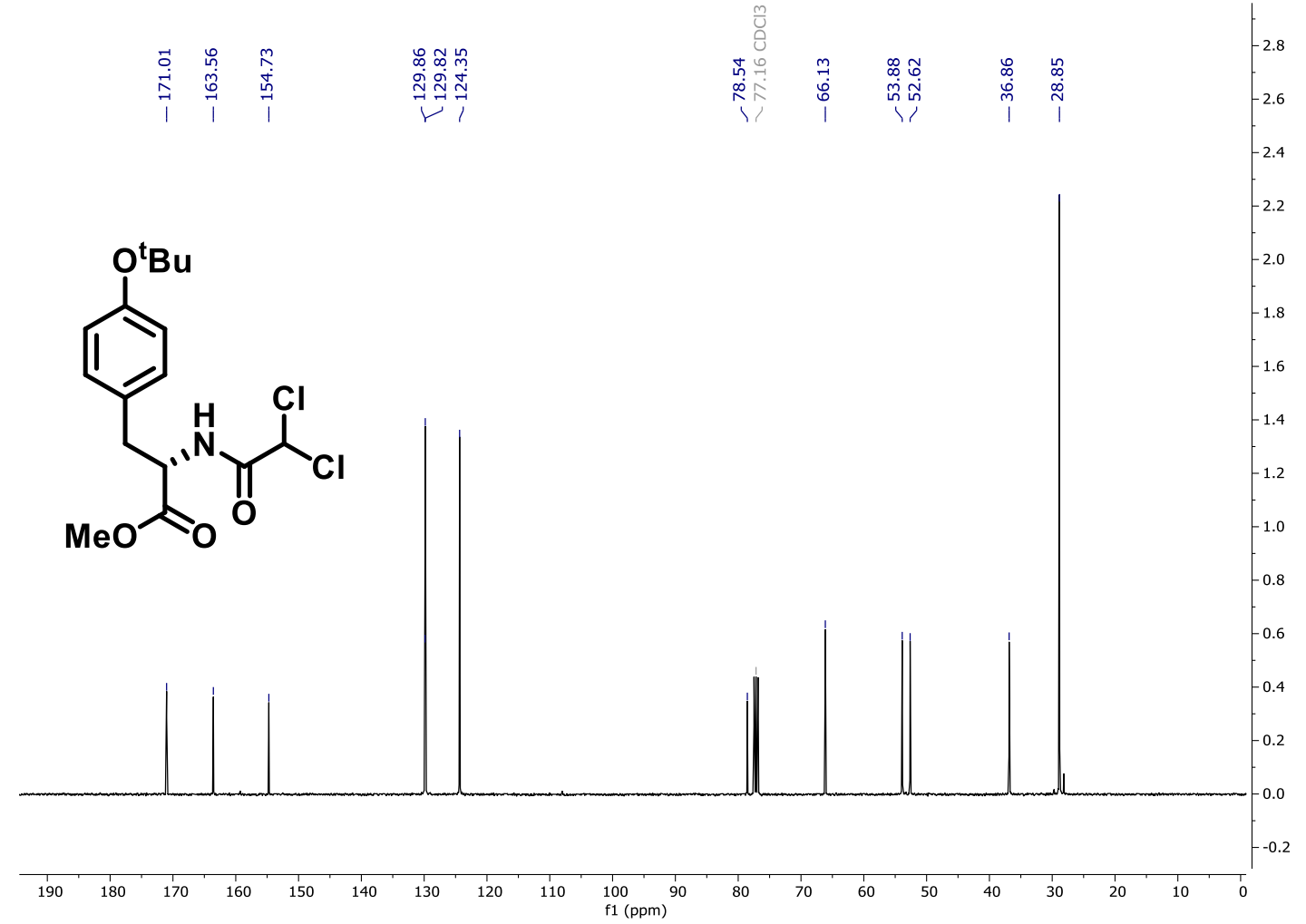


2,2-Dichloro-1-(piperidin-1-yl)ethan-1-one 3ak

${ }^{1} \mathrm{H}$ NMR (400 MHz, $\left.\mathrm{CDCl}_{3}\right)$

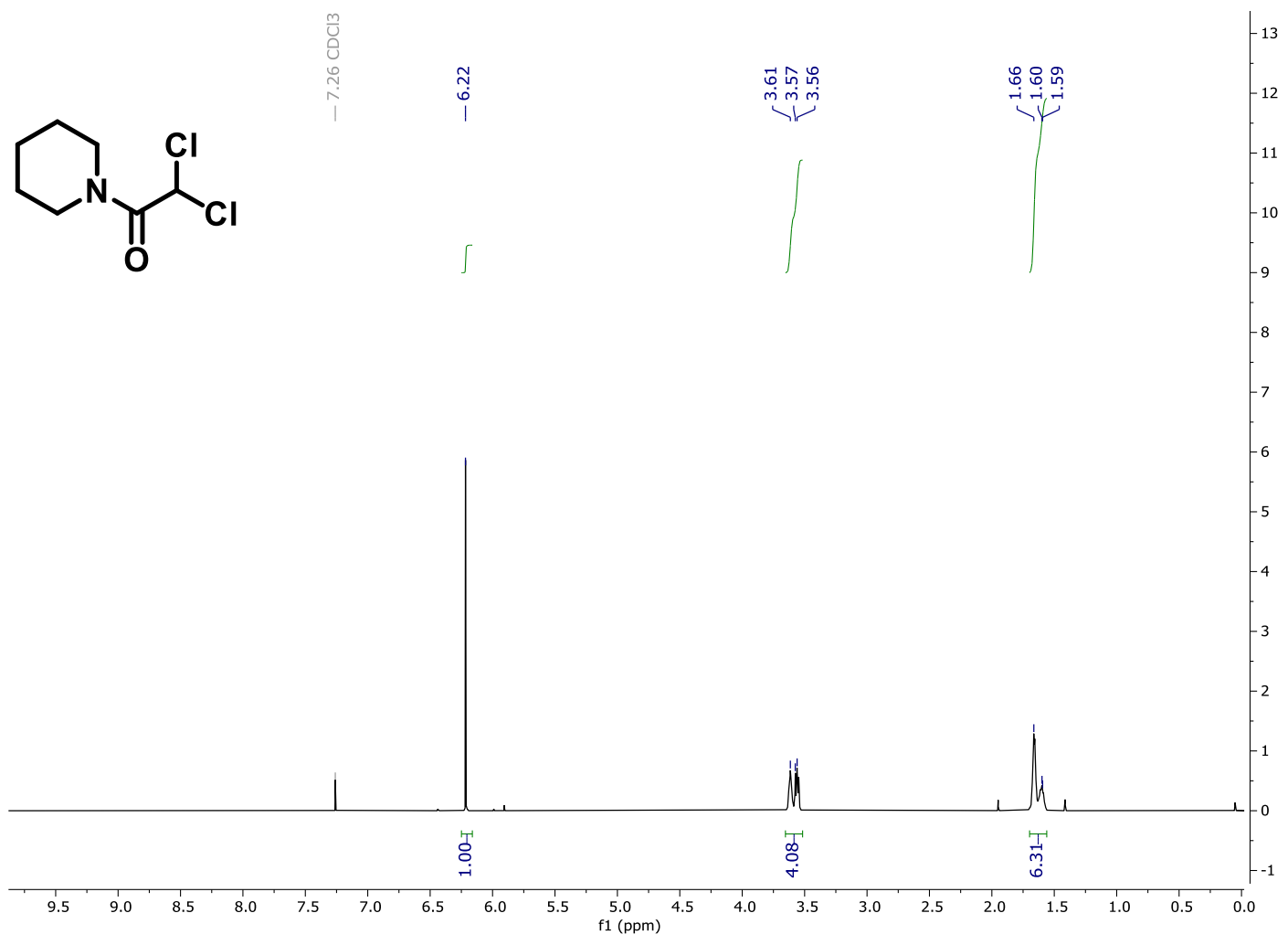


${ }^{13} \mathrm{C}$ NMR (101 MHz, $\left.\mathrm{CDCl}_{3}\right)$

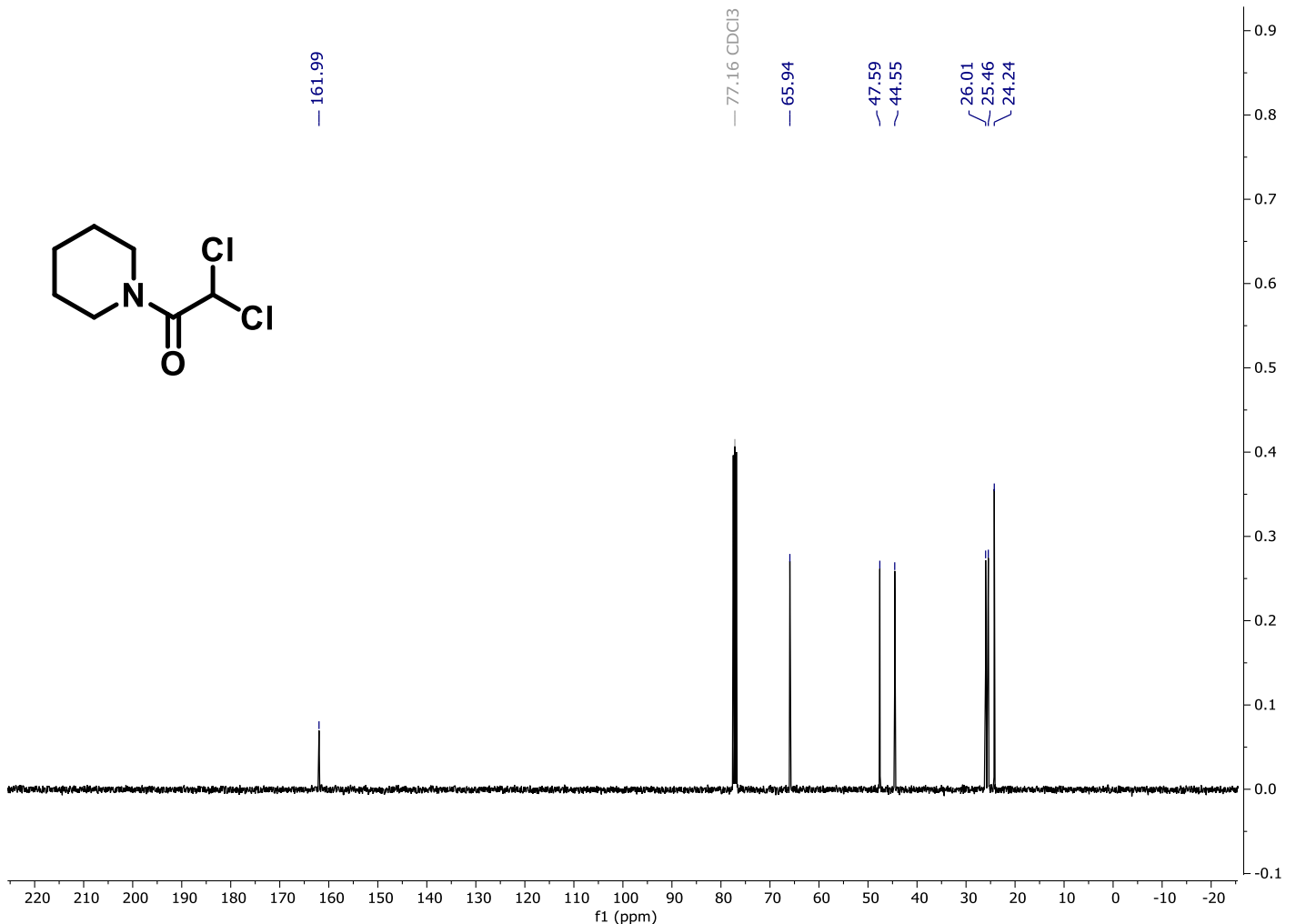


DL-phenylalanine ethyl ester rac-1ai

${ }^{1} \mathrm{H}$ NMR (400 MHz, $\mathrm{CDCl}_{3}$ )

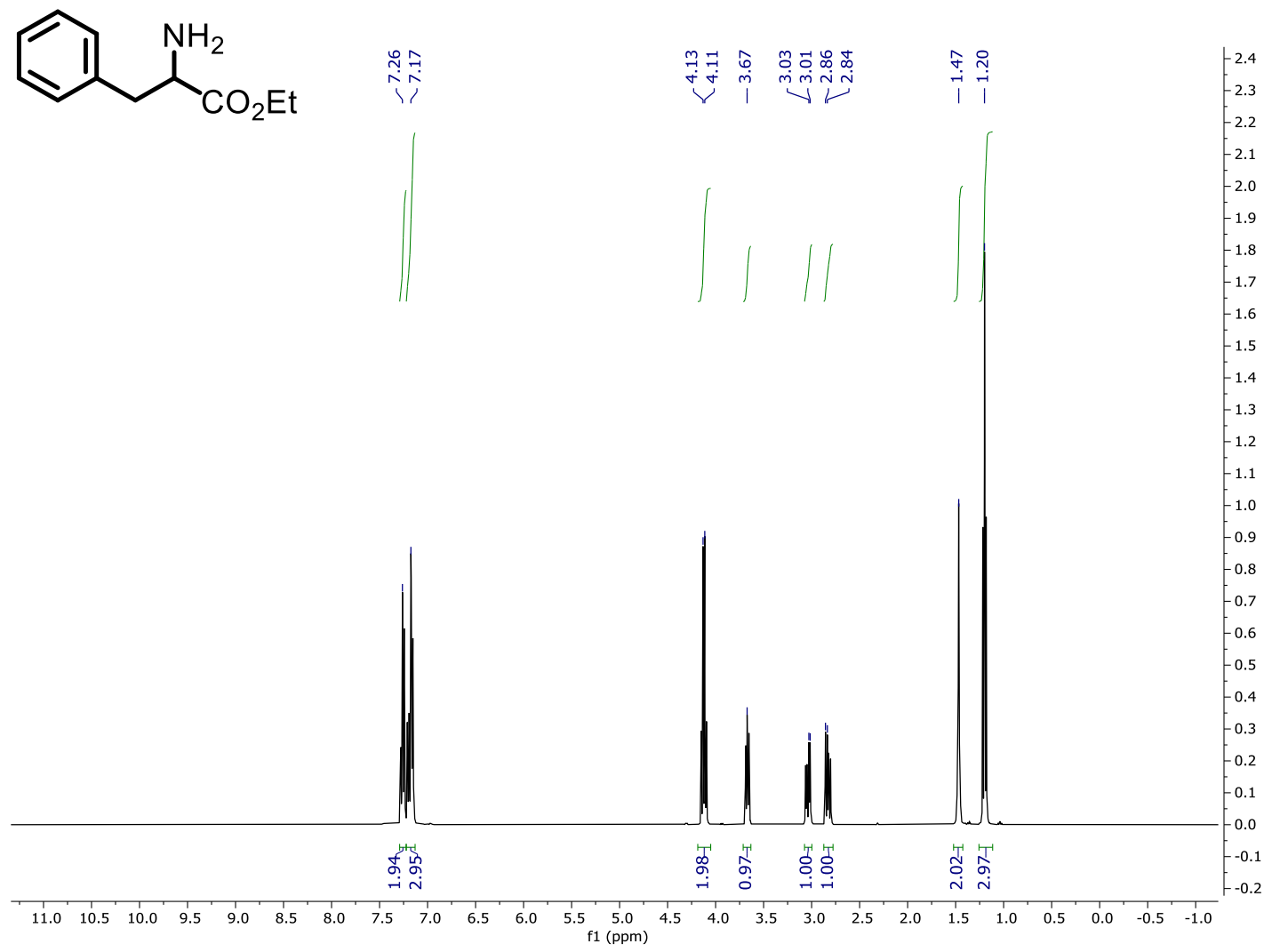


${ }^{13} \mathrm{C}$ NMR (101 MHz, $\mathrm{CDCl}_{3}$ )

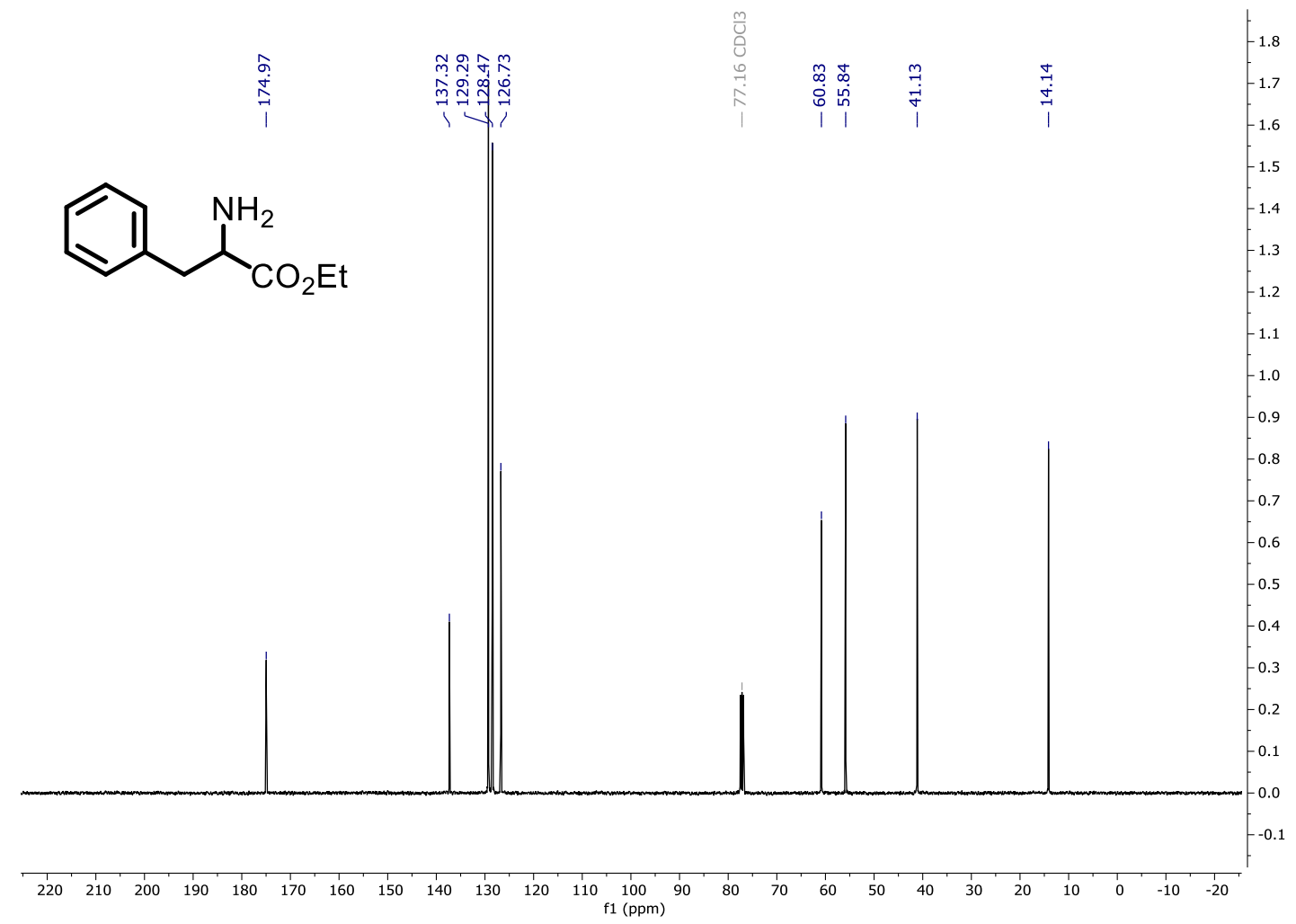




\section{References}

[1] W. C. Still, M. Kahn, A. Mitra, J. Org. Chem. 1978, 43, 2923-2925.

[2] J. Tao, T. N. Tuck, G. K. Murphy, Synth. 2016, 48, 772-782.

[3] Z. Ke, Y. P. Lam, K. S. Chan, Y. Y. Yeung, Org. Lett. 2020, 22, 7353-7357.

[4] W.-B. Liu, C. Chen, Q. Zhang, Z.-B. Zhu, Beilstein J. Org. Chem. 2012, 8, 344-348.

[5] Y. Yang, P. Shang, C. Cheng, D. Wang, P. Yang, F. Zhang, T. Li, A. Lu, Y. Zhao, Eur. J. Med. Chem. 2010, 45, 4300-4306.

[6] A. Pähler, G. Birner, J. Parker, W. Dekant, Chem. Res. Toxicol. 1998, 11, 995-1004.

[7] H. Lundberg, F. Tinnis, H. Adolfsson, Chem. - A Eur. J. 2012, 18, 3822-3826.

[8] M. Fereidoonnezhad, S. M. H. Tabaei, A. Sakhteman, H. Seradj, Z. Faghih, Z. Faghih, A. Mojaddami, B. Sadeghian, Z. Rezaei, J. Mol. Struct. 2020, 1221, 128689.

[9] V. Pace, L. Castoldi, A. D. Mamuye, W. Holzer, Synth. 2014, 46, 2897-2909.

[10] E. Manouchehrizadeh, A. Mostoufi, E. Tahanpesar, M. Fereidoonnezhad, Pharm. Chem. J. 2020, 54, 148-153.

[11] K. L. Amsberry, R. T. Borchardt, J. Org. Chem. 1990, 55, 5867-5877.

[12] I. V. Kapitanov, A. Jordan, Y. Karpichev, M. Spulak, L. Perez, A. Kellett, K. Kümmerer, N. Gathergood, Green Chem. 2019, 21, 1777-1794.

[13] J. S. Witzeman, W. D. Nottingham, J. Org. Chem. 1991, 56, 1713-1718.

[14] R. A. Sheldon, Green Chem. 2007, 9, 1273-1283. 